\title{
Interventional Techniques in the Management of Chronic Pain: Part 2.0
}

\author{
Laxmaiah Manchikanti, MD, Vijay Singh, MD, David Kloth, MD, Curtis W. Slipman, MD, \\ Joseph F. Jasper, MD, Andrea M. Trescot, MD, Kenneth G. Varley, MD, Sairam L. Atluri, MD, \\ Carlos Giron, MD, Mary Jo Curran, MD, Jose Rivera, MD, A. Ghafoor Baha, MD, Cyrus E. Bakhit, MD \\ and Merrill W. Reuter, MD
}

The practice guidelines for interventional techniques in the management of chronic pain are systematically developed statements to assist physician and patient decisions about appropriate health care related to chronic pain. These guidelines are professionally derived recommendations for practices in the diagnosis and treatment of chronic or persistent pain. They were developed utilizing a combination of evidence and consensus based techniques, to increase patient access to treatment, improve outcomes and appropriateness of care, and optimize cost-effectiveness. The guidelines include a discussion of their purpose, rationale, and importance, including the patient population served, the methodology and the pathophysiologic basis for intervention. Various interventional techniques will be discussed addressing the rationale for

Practice guidelines in various formats have been a part of medical practice for well over 50 years, and by some accounts over 150 years (1-14). Clinical practice guidelines are “. . . s systematically developed statements to assist practitioner and patient decisions about appropriate health care for specific clinical circumstances" (1). These are professional practice recommendations for practices for prevention, diagnosis and treatment of acute and chronic painful disorders, and in some cases, disability management. Guidelines have existed for centuries, in forms ranging from Sanskrit and Greek protocols and folk medicine practices, to rigorous, scientifically tested algorithms (1,

From the American Society of Interventional Pain Physicians, Paducah, Kentucky. Authors are members of American Society of Interventional Pain Physicians. Appendix I describes author affiliations. Address correspondence: Laxmaiah Manchikanti, MD, 2831 Lone Oak Road, Paducah, KY 42001. Email: $\underline{\text { drm@asipp.org }}$ their use in chronic pain with analysis of the outcomes data and cost effectiveness.

These guidelines do not constitute inflexible treatment recommendations. It is expected that a provider will establish a plan of care on a case-by-case basis, taking into account an individual patient's medical condition, personal needs, and preferences, and the physician's experience. Based on an individual patient's needs, treatment different from that outlined here could be warranted.

Keywords: Interventional techniques, neural blockade, chronic pain, epidural injections, percutaneous epidural adhesiolysis, discography, facet joint mediated pain, radiofrequency

2 ). There has been a rapid increase in guideline development since 1985, in part propelled by the realization that medical practices for similar conditions vary widely among geographic areas, specialties, and countries (1, 2, 15-20). In recent years, practice guidelines have become more prominent to improve the quality of health care, protection of professional autonomy, reduction of litigation risk, minimization of practice variation, provision of standards for auditing medical records, reduction of health care costs, defining areas of practice, improvement in efficiency of practice, and identification of inappropriate care $(4,9,12$, 21-26).

Shaneyfelt et al (27) reviewed the methodological quality of clinical guidelines in the peer-reviewed medical literature, with evaluation of 279 guidelines developed by 69 different organizations and published from 1985 to 1997. It is expected that the guidelines published by various organizations and not included in the peer-reviewed literature are nearly 2500 in the US alone. Of significant importance are the various guidelines developed by the 
Agency for Health Care Policy and Research (AHCPR), which replaced the National Center for Health Services Research and Health Care Technology Assessment (NCHSR) in 1989 (28). The Agency for Health Care Policy and Research developed approximately 15 guidelines, with a budget of $\$ 750$ million. The guidelines developed by AHCPR for managing acute low back pain are of significant importance for practice of interventional pain medicine (28). Other guidelines of significance for pain specialists include those developed by the Quebec Task Force in the management of spinal disorders and whiplash associated disorders $(29,30)$, chronic pain management guidelines by the American Society of Anesthesiologists (31), guidelines for performance of facet joint blocks by the International Spinal Injection Society (32), and interventional techniques in the management of chronic pain by the Association of Pain Management Anesthesiologists (33). Additionally, there are guidelines for migraine headaches (34), guidelines for managing pain in sickle cell disease (35), chronic pain management guidelines in the elderly (36), and guidelines the for management of chronic pain syndrome $(37,38)$. Other guidelines include the ones developed by local Medicare carriers in the form of local Medicare Review Policies in various states; third-party payors including Blue Cross and Blue Shield, Aetna and others; entrepreneurial technological companies such as Hayes Technologies; and position statements by a multitude of individuals and organizations. Cochrane collaboration back review group for spinal disorders was also started in $1995(39,40)$. McQuay and Moore published a book of evidence based resource for pain relief (3). Many of these guidelines were developed at a cost of tremendous effort and resources to review the assessment and treatment literature and to develop evidence-based guidelines to treat various conditions. However, the cost of the guidelines by various organizations is much less than the ones developed by the federal government by the AHCPR. A serious examination of the guidelines shows that about $85 \%$ of the recommendations are not based on any significant evidence (27). Interventional pain management is no exception to the general rule.

\section{CLINICAL PRACTICE GUIDELINES}

\section{Purpose}

Clinical practice guidelines for interventional techniques in the management of chronic pain are professionally developed utilizing a combination of evidence, expert opinion and consensus. The purpose of these clinical guidelines is to:
1. Improve quality of care,
2. Improve patient access,
3. Improve patient outcomes,
4. Improve appropriateness of care,
5. Improve efficiency and effectiveness, and
6.
$\quad$ Achieve cost containment by improving cost-ben-

\section{Rationale}

The most compelling single reason for the development of these clinical practice guidelines is to improve the quality of care and life for patients suffering from painful disorders. Available evidence documents a wide degree of variance in the practice of interventional pain management and pain medicine for even the most commonly performed procedures and treated condition(s) $(6,23-38,41-63)$. These guidelines also address the issue of systematic evaluation and ongoing care of chronic or persistent pain, and provide information about the scientific basis of recommended procedures, thus potentially increasing compliance, dispelling misconceptions among providers and patients, managing patient expectations reasonably, and forming the basis of a therapeutic partnership among the patient, the provider, and the payer.

\section{Importance}

Interventional techniques are crucial both in the diagnostic, as well as the therapeutic, arena of managing pain and providing improvement in the quality of life of the pain sufferers. Some insurance carriers and other medical specialties have criticized the practice of interventional pain medicine and pain management using the wide variations in treatment protocols and the relative scarcity of conclusive evidence or consensus for their justification.

\section{Methodology}

The two most common methods for the development of guidelines, often combined, are based on evidence and consensus. However, reviews, clinical decision analyses, and economic analyses are also very commonly utilized in the medical literature. Thus, clinicians are increasingly being asked to remain current in the aspects of clinical care and decision making by systematically gathering, analyzing, and combining evidence that links to outcome (6). However, many of these publications unfortunately do not always link information in a direct way to clinical recommendations $(3-5,24,25,64)$. Implicit in the definition of 
clinical practice guidelines is that they not only be systematically and scientifically developed but also should be able to assist practitioner and patient in making real life clinical decisions. The Institute of Medicine (IOM) implicitly incorporates rigorous science-based procedures as a part of the development of practice guidelines and decision making includes both clinicians and patients with a focus on specific clinical circumstances, without direction toward technology or procedures (6). The American Medical Association (AMA) uses the term practice parameter and defines this practice as “. . . strategies for patient management, developed to assist physicians in clinical decision making. Practice parameters are highly variable in their content, format, degree of specificity, and method of development" (65). Thus, the methods that are used to develop practice guidelines vary among organizations and depend on objectives of the guideline and philosophic approach.

Methods of development are classified as informal consensus development, formal consensus development, evidence-based guideline development, and explicit guideline development $(5-7,10,65)$. However, a combination of multiple approaches is commonly utilized. Evidencebased guideline development provides a link between the strength of recommendations and the quality of evidence. Even though this approach may seem to have enhanced the scientific rigor of guideline development, recommendations may not always meet the highest scientific evidence (27).

Evidence-based practice originated in the 50s with the advent of randomized, controlled trials. A randomized, controlled trial, also known as RCT, is a trial in which participants are randomly assigned to two groups: first, (experimental group) receiving the intervention that is being tested, and the other (the comparison or control group) receiving an alternative treatment or placebo. This design allows assessment of the relative effects of interventions. It is presumed that the strident debate between the proponents and opponents of evidence-based medicine has led to clarity (7). The current evidence-based medicine is defined as the conscientious, explicit, and judicious use of current best evidence in making decisions about the care of individual patients (10). The practice of evidence-based medicine requires the integration of individual clinical expertise with the best available external clinical evidence from systematic research. It should be construed that, apart from the results of the randomized controlled trials, there are many other factors that may weigh heavily in both clinical and policy decisions, such as patient preferences and resources, and these must contribute to decisions about the care of the patients (7). Thus, all evidence should be considered and no one sort of evidence should necessarily be the determining factor in a decision. There are an increasing number of well-conducted randomized, controlled trials and systematic reviews. However, such studies are difficult to conduct in chronic pain management with interventional procedures as well as surgical procedures. Clinical trials of the efficacy of commonly used interventions in low back pain were reviewed by Koes and coworkers (66), and Tulder and coworkers (67), which led to the conclusion that the methodological quality in these studies was disappointingly low. Similar conclusions were drawn in other evaluations (27-30). The quality of meta-analytic procedures in chronic pain treatment also has been questioned (68). In addition, the issues of ethics, feasibility, cost and reliability pose challenges to the randomized trial, specifically in surgical settings and treatments involving interventional procedures (69-75). Most of the studies of interventional pain procedures have been performed by multiple specialty groups (rarely including pain specialists) and without radiographic control, especially in the case of epidural steroid injections.

Concato et al (76) conducted a study of randomized, controlled trials, observational studies, and hierarchy of research designs. They described that, in the hierarchy of research designs, the results of randomized, controlled trials have been considered to be evidence of the highest grade, whereas observational studies have been seen as having less validity because such studies reportedly overestimate treatment effects. Concato et al (76) showed that the average results of the observational studies were remarkably similar to those of the randomized, controlled trials, and concluded that the results of well-designed observational studies (with either a cohort or a case-controlled design) do not systematically overestimate the magnitude of the effects of treatment as compared with those in randomized, controlled trials on the same topic. However, this is not to say that we do not need randomized, controlled studies. Pocock and Elbourne (77) observed that, in a systematic review of evidence on a therapeutic topic, one needs to take into account the quality of the evidence, since in any randomized or observational study, bias may exist either in design or analysis. The importance of the difficulty of a large randomized trial with interventional procedures is reinforced by the failure to complete a randomized, controlled trial to evaluate epidural steroid injections, which was funded by the American Society of Regional Anesthesia as a Koller Award (78). In addition, Turk (79) suggests that it is important to acknowledge that 
Table 1. Type and strength of efficacy evidence

\begin{tabular}{|c|c|c|}
\hline Level & Type of strength & Description of evidence \\
\hline I & Conclusive & Research-based evidence with multiple relevant and high-quality scientific studies \\
\hline II & Strong & $\begin{array}{l}\text { Research-based evidence from at least one properly designed randomized, } \\
\text { controlled trial of appropriate size (with at least } 60 \text { patients) and high-quality or } \\
\text { multiple adequate scientific studies }\end{array}$ \\
\hline III & Moderate & $\begin{array}{l}\text { Evidence from well-designed trials without randomization, single group pre-post } \\
\text { cohort, time series, or matched case-controlled studies }\end{array}$ \\
\hline IV & Limited & $\begin{array}{l}\text { Evidence from well-designed nonexperimental studies from more than one center } \\
\text { or research group }\end{array}$ \\
\hline $\mathrm{V}$ & Indeterminate & $\begin{array}{l}\text { Opinions of respected authorities, based on clinical evidence, descriptive studies, } \\
\text { or reports of expert committees }\end{array}$ \\
\hline
\end{tabular}

statistical significance and clinical significance are not necessarily equal and that there might be disagreements concerning how to judge the clinical significance of each study. Schulz et al (80) in describing the empirical evidence of bias, estimated that lack of randomization may overestimate the treatment effect by $30 \%$ to $41 \%$; whereas if the study is not double-blind, overestimation may be approximately $17 \%$. Of course, the study by Concato et al (76) disputes this assertion.

The publication of randomized, controlled trials concerning pain have increased significantly. However, only $14 \%$ of these studies were of invasive procedures, on the other hand, $54 \%$ of all reports were in acute pain, whereas $43 \%$ were in chronic noncancer pain, and $3 \%$ were in cancer pain $(81,82)$. The Agency for Health Care and Policy Research (28) described evidence rating for management of acute low back pain problems in adults. The AMA, office of quality assurance, also described five attributes for the development of practice parameters (65). The Institute of Medicine (6) described several attributes to the guideline content and guideline development, while McQuay and Moore (3, 7), and others (39, 40, 45, 66, 67) described type and strength of efficacy evidence. For the purpose of development of these guidelines, a blended approach for type and strength of efficacy evidence categorized into five types was utilized in Table 1.

Thus, in the development of these clinical guidelines of interventional techniques in managing chronic pain all applicable standards for evidence rating were utilized. Due to the poor methodological quality of a large number of published randomized clinical trials on the efficacy of in- terventions in the management of low back pain, whiplash, and other painful conditions, even though the focus of these guidelines was on evidence that consisted of studies of randomized, well-controlled studies, other evidence was also utilized which included reports of meta-analysis and high quality observational studies with adequate size. Consequently, the focus of these guidelines is physiological, supported by peer-reviewed literature, based on the best cost-benefit balance for the patient both in the short and long term, expert opinion(s) and consensus.

\section{Population}

The population covered by these guidelines includes all patients suffering with chronic pain of either spinal or nonspinal origin eligible to undergo interventional technique(s).

\section{IMPLEMENTATION AND REVIEW}

The dates for implementation and review were established:

$\begin{array}{ll}-\quad \text { Effective date - February 1, } 2001 \\ \quad & \text { Expiration date - January 31, } 2003 \\ & \text { Scheduled review - July 1, 2002 }\end{array}$

\section{CONTROVERSIES}

Controversial aspects of guidelines range from the difference between a guideline and a pathway, targeted patient population, the definition of evidence, variability and evaluation of strength of studies and final conclusions of evidence, author bias, and finally the special interest group 
influences. Practice guidelines are systematically developed statements to assist practitioner and patient decisions about appropriate health care for specific clinical circumstances. In contrast, clinical pathways are tools to coordinate the time-dependent progress of a typical uncomplicated patient across many clinical departments specific to the condition or disease being managed (64). The differences between target patient population ranges from specific conditions such as acute low back pain, migraine headaches, sickle-cell disease, and complex pain syndromes, to much more general guidelines applicable across a variety of chronic painful conditions. The next issue of controversy and contention is the definition of evidence. All of the guidelines incorporate literature review. However, the categorization of evidence of strength differs across guidelines. Generally, evidence of strength ranges from prospective, double-blind, randomized, controlled studies to uncontrolled case reports. In addition to the evidence, panels also attempt to use expert consensus, the application of which varies across the development of the guidelines. Each panel developing the guidelines feel that their guidelines applied the most stringent and reasonable evidence. On the other hand, one group developing the guidelines tend to criticize another group when they differ philosophically $(3,7,21,24,25,27-39,45,46,62,64-67,83$, $84)$. In addition, the same evidence may be evaluated by different groups or authors with variability interpretation of results. Author bias also exists regardless of the desire to achieve substantially impartial, scientifically based recommendations. It is unavoidable that guidelines reflect authors' clinical and practice biases, personal philosophy, and the way the literature is interpreted. Certainly on the same spectrum, influences of the special interest groups are inescapable.

\section{CHRONIC PAIN}

"We must all die. But that I can save him from days of torture, that is what $I$ feel as my great and ever new privilege. Pain is a more terrible Lord of mankind than even death itself."

- Albert Schweitzer

Schweitzer (85), the great humanitarian, physician, and Nobel laureate, elegantly described the nature of pain and the obligation and privilege of the physician and other health professionals to relieve it in 1931, after nearly two decades of experience of medical practice in the African jungle. Approximately four decades later in 1974, John Bonica, the father of pain medicine, observed: "Pain is the most pressing issue of modern times." Today, in the new millennium as then, proper management of pain remains one of the most important and most pressing issues of society in general and the scientific community in the health professions in particular.

\section{Epidemiology}

In spite of the best efforts of the public, providers and the government, pain continues to be an epidemic $(86,87)$. In addition, inadequate treatment of pain also continues to be a public health problem, that is reaching epidemic proportions in the United States and across the world (86-96). The knowledge and understanding of this complex entity, including diagnosis and treatment, are in infancy, in spite of modern developments in medicine. Providers, patients, and the government all understand the devastating nature of chronic pain which destroys the quality of life by eroding the will to live, disturbing sleep and appetite, creating fatigue, and impairing recovery from illness or injury (86100). In elderly patients it may make the difference between life and death by resulting in vocational, social, and family discord (100-105). Pahor et al (102) found that pain relief is particularly elusive for older women with disabling back and lower extremity problems. In this study, approximately two thirds of the women reported significant levels of pain and difficulty in controlling it. Asch et al (106) measured underuse of necessary care detecting substantial underuse problems for various conditions, including depression, and concluded that these problems likely result in negative outcomes in the elderly population.

The concept of chronic pain is beset with controversy, starting with its very definition. For some chronic painful conditions, it is defined as, "pain that exists beyond an expected time frame for healing." For other conditions, it is recognized that, "healing may never occur." Bonica defined chronic pain as, "Pain which persists a month beyond the usual course of an acute disease or a reasonable time for any injury to heal that is associated with chronic pathologic processes that causes a continuous pain or pain at intervals for months or years" (107). In many cases, chronic pain is understood as persistent pain that is not amenable to routine pain control methods. In a Gallup Survey of "pain in America" more than 4 out of 10 adults (42\%) say they experience pain on a daily basis (108). Americans age 65 and older are more likely to experience pain for longer periods of time than younger Americans (108). Andersson et al (109) reported incidence of persistent pain for 6 months in $49 \%$ of the adult population, with functional disability in $13 \%$. Perquin et al (97) reported 
Table 2. Prevalence of low back and neck pain in general population ranked by severity and disability

\begin{tabular}{lcc}
\hline Pain grade & Low back pain & Neck pain \\
\hline Grade I & $47 \%$ & $39 \%$ \\
Low pain intensity and disability & $12 \%$ & $9 \%$ \\
Grade II & & $5 \%$ \\
High pain intensity and low disability & $13 \%$ & $5 \%$ \\
Grades III and IV & & $\mathbf{5 3 \%}$ \\
High pain intensity with moderate and severe disability & $\mathbf{7 2 \%}$ & \\
\hline Total & & \\
\hline
\end{tabular}

Data modified and adapted from Cassidy et al (118) and Côté et al (119)

that chronic pain is a frequent complaint even in childhood and adolescence. The International Association for the Study of Pain (IASP) appointed task forces to study the epidemiology of pain in 1996 (110), and pain in the elderly in 1999 (111) with publication of two large reports of 137 and 320 pages. It was consistently shown that elderly suffered with not only pain of longer duration, but with higher frequency (100-105, 108, 111-116).

Among the chronic pain problems, spinal pain which includes pain emanating from cervical, thoracic and lumbosacral regions constitutes the majority of the problems. It is estimated that episodes of low back pain that are frequent or persistent have been reported in $15 \%$ of the US population, with a lifetime prevalence of $65 \%$ to $80 \%$ (103, 113). However, prevalence of neck pain, though not as common as low back pain, is estimated $35 \%$ to $40 \%$ (114, $115)$, of which $30 \%$ will develop chronic symptoms (116).
In contrast, the epidemiological data in relation of thoracic pain support the view that the thoracic spine is less commonly involved. Linton et al (117) estimated prevalence of spinal pain in the general population as $66 \%$, with only $15 \%$ of those reporting thoracic pain; in comparison to $56 \%$ to $44 \%$ for the lumbar and cervical regions respectively. Cassidy and colleagues (118) assessed the 6-month prevalence of chronic low back pain and its impact on general health in the Canadian population. The results showed an $84 \%$ lifetime prevalence, with $47 \%$ of the patients reporting grade I pain (low pain intensity and low disability); $12 \%$ grade II pain (high pain intensity and low disability); $13 \%$ grade III (high pain intensity/moderate disability), and grade IV (high pain intensity/severe disability) (Table 2). They also reported that grade I low back pain was more common in the younger population while older age groups reported higher incidence of grade III/IV pain. Thus, a total $13 \%$ of the population suffers with high

Table 3. Chronicity of low back pain

\begin{tabular}{lccc}
\hline & \multicolumn{3}{c}{ Prevalence } \\
\hline Author(s) & Year of Publication & 3 months & 12 months \\
\hline Anderson and Svensson (122) & 1983 & $20 \%$ & $10 \%$ \\
Van Den Hoogen et al (123) & 1997 & $35 \%$ & $35 \%$ \\
Croft et al (124) & 1998 & $79 \%$ & $75 \%$ \\
Carey et al (125) & 1999 & N/A & $20 \%$ to $35 \%$ \\
Meidema et al (126) & 1998 & N/A & $28 \%$ \\
Thomas et al (127) & 1999 & $48 \%$ & $42 \%$ \\
\hline
\end{tabular}

$\mathrm{N} / \mathrm{A}=$ Not available 


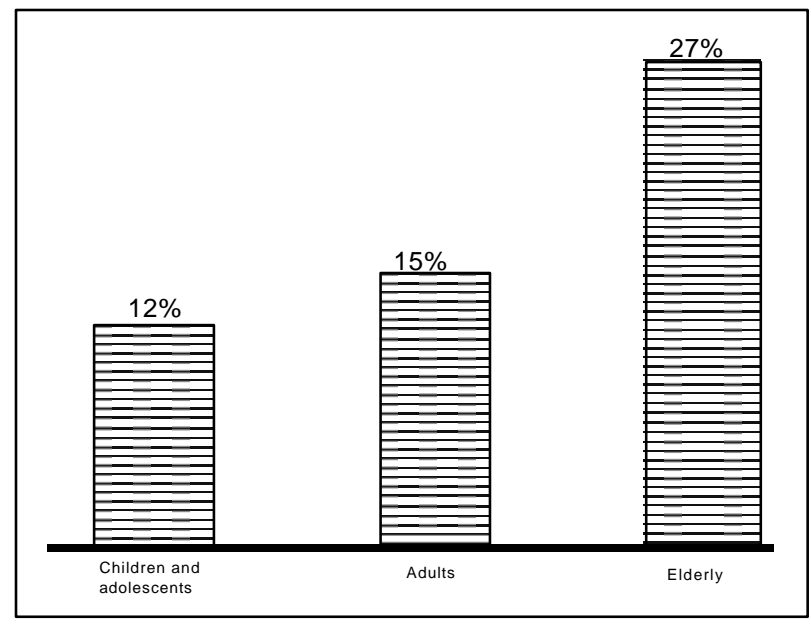

Fig. 1. Estimated average of age related prevalence of low back pain

Reproduced with permission from Manchikanti (103)

pain intensity with moderate or severe disability, whereas an additional $12 \%$ suffer with high pain intensity but with a low disability. In a similar study, Cote et al $(119,120)$, evaluating neck pain and its related disability, reported that overall, $39 \%$ of the sample experienced grade I neck pain, whereas $9 \%$ experienced grade II neck pain, and 5\% had grade III and IV neck pain (Table 2). Almost 16\% of the respondents reported having previously injured the neck in a motor vehicle collision $(119,120)$.

Duration of back pain and its chronicity have been a topic of controversy. It is believed that most of these episodes will be short-lived, with $80 \%$ to $90 \%$ of attacks resolving in about 6 weeks irrespective of the administration or type of treatment; and $5 \%$ to $10 \%$ of patients developing persistent back pain $(121,122)$. However, this concept has been questioned, as the condition tends to relapse, so most patients will experience multiple episodes. As shown in Table 3, prevalence of low back pain ranged from $35 \%$ to $79 \%$ at 3 months and $35 \%$ to $75 \%$ at 12 months (123-127). The studies evaluating the chronicity of low back pain estimated the average of age related prevalence of persistent low back pain as $12 \%$ in children and adolescents, $15 \%$ in adults, and $27 \%$ in the elderly (Fig. 1). Bressler and colleagues (101), in a systematic review of the literature determined that overall prevalence of low back pain in the elderly was $27 \%$ derived from a total elderly population base of 17,173 with reports from 12 studies from community population, primary care settings and from the nursing homes with prevalence ranging from $13 \%$ to $51 \%$ (101, 109, 128-138).

\section{Chronic Pain vs Chronic Pain Syndrome}

Two major and controversial terms in today's pain medicine are "chronic pain," also known as persistent pain, and a second category known as "chronic pain syndrome," which is a separate and distinct condition (139-142). Chronic pain or persistent pain persists beyond the expected healing time of an injury or an illness, usually considered beyond 6 months. Chronic pain may be associated with psychological problems such as depression, generalized anxiety disorder, and some behavioral problems. However, chronic pain improperly diagnosed or inadequately treated can result in deteriorating coping skills and limitations and reduction in functional capacity. In contrast, chronic pain syndrome is a complex condition with physical, psychological, emotional, and social components (141, 142). Both chronic pain and chronic pain syndrome can be defined in terms of duration and persistence of the sensation of pain, and presence or absence of psychological and emotional components. However, chronic pain syndrome, as opposed to chronic pain, has the added component of certain recognizable psychological and socioeconomic influences, with characteristic psychological and sociological behavior patterns inherent in chronic pain syndrome that distinguish the two conditions (141). According to the fifth edition of Guides to Evaluation of Permanent Impairment published in 2000 (142), the term chronic pain syndrome even though not official nomenclature, is frequently used to describe an individual who is markedly impaired by chronic pain with substantial psychological overlay. The guides (142) also state that chronic pain syndrome is largely a behavioral syndrome that affects a minority of those with chronic pain. It may best be understood as a form of an abnormal illness behavior that consists mainly of excessive adoption of the sick role. The guides also caution that while the term is useful in certain situations, it does not, however, substitute for a careful diagnosis of physiologic, psychological, and conditioning components that comprise the syndrome. The term chronic pain syndrome must be used with caution, as grouping pain problems together under a general disorder may mask and leave untreated important physiologic differences (142). Thus, chronic pain may exist in the absence of chronic pain syndrome, but chronic pain syndrome always presumes the presence of chronic pain. The terminology recommended by IASP has eliminated chronic pain syndrome from the glossary (140). The IASP Task Force on Taxonomy on classification of chronic pain describing definitions of pain terms described that it is common in North America to find patients as having "chronic pain syndrome" (140). In this case, the Task Force believed that the words 
are being used as a diagnosis that usually implies a persistent pattern of pain that may have arisen from organic causes but which is now compounded by psychological and social problems resulting in behavioral changes. Even though the Task Force was asked to adopt such a label, particularly for use in billing in the United States, there was general agreement in the Task Force that this would not be desirable. The Task Force also noted that the term "chronic pain syndrome" is often, unfortunately, used pejoratively (140). However, the literature shows that chronic pain syndrome is not a common phenomenon in general, and it is particularly very infrequent in the elderly (143). In addition, Hendler et al (144), to whom a number of suspected "psychosomatic" cases have been referred, found organic origin of the pain in $98 \%$ of cases. Subsequently, Hendler and Kolodny (145) estimated that the incidence of psychogenic pain is only 1 in 3000 patients.

Chronic pain has been estimated to cost the American society approximately $\$ 120$ billion a year in treatment, lost revenues, and wages. Some frightening estimates show that annual total costs for back pain itself, including disability and litigation, are more than $\$ 100$ billion (146). Annual direct medical costs for back pain are estimated at around $\$ 33$ billion, with chronic pain around $\$ 45$ billion. Approximately $28 \%$ to $30 \%$ of the US population suffer with some kind of chronic painful condition(s).

\section{PATHOPHYSIOLOGIC BASIS}

Spinal pain is inclusive of all painful conditions originating from spinal structures ranging from the discs to muscles and ligamentous attachments. In contrast, nonspinal pain encompasses a multitude of other painful conditions, ranging from peripheral neuralgias to reflex sympathetic dystrophy and arthritis. Any structure with a nerve supply capable of causing pain similar to that seen in clinically normal volunteers, which is susceptible to diseases or injuries that are known to be painful, can cause pain $(32,33$, $41,42,56,147-185)$. For a structure to be implicated, it should have been shown to be a source of pain in patients, using diagnostic techniques of known reliability and validity $(32,33,41,42,151-169)$. The structures responsible for pain in the spine include the vertebrae, intervertebral discs, spinal cord, nerve roots, facet joints, ligaments, and muscles $(32,33,41,42,147-165,168,170-185)$. Similarly, muscles, ligaments, various joints including (atlantooccipital joints, atlantoaxial joints, and sacroiliac joints), sensory nerves, the sympathetic nervous system, and visceral organs have been implicated in pain of nonspinal origin $(166,167,169,186-215)$.
Facet joints have been implicated as responsible for spinal pain in $15 \%$ to $45 \%$ of patients with low back pain (178183 ) and $54 \%$ to $60 \%$ of patients with neck pain utilizing controlled diagnostic blocks $(184,185)$. The degeneration of the disc resulting in primary discogenic pain is seen commonly with or without internal disc disruption alleged to be the number one cause of spinal pain $(162,163,174$, 180, 216-260). Disc degeneration is a well accepted sequela of the normal aging process, particularly of the lower lumbar levels. Kirkaldy-Willis et al (216) described the pathogenesis of degenerative changes in the aging spine entailing three phases, whereas Handel et al (217) described a structural degenerative cascade for the cervical spine with four phases. In this model, degenerative cascade is viewed in a context of a three-joint complex, with involvement of changes in the disc structure and composition paralleling changes in the articular cartilage and ligaments of joints. Internal disc disruption has been considered as a commonly overlooked source of chronic low back pain $(163,226)$. In fact, a controlled study reported the prevalence of pain due to internal disc disruption as $39 \%$ in patients suffering with chronic low back pain (174). The prevalence of cervical discogenic pain in patients with chronic neck pain of traumatic origin was shown to be $61 \%$ (162). However, the prevalence of cervical discogenic pain has not been formally studied. In contrast, disc herniation is seen in a small number of patients ranging from $4 \%$ to $6 \%$ (163, 173, 222-230, 255-260).

Postlaminectomy syndrome or pain following operative procedures of the spine is also becoming a common entity in modern medicine (261-292). Although the exact incidence and prevalence of postlaminectomy syndrome is not known, it is estimated that $20 \%$ to $30 \%$ of spinal surgeries (occasionally as high as 40\%), may not be successful as a result of either the surgery being inadequate, incorrect, or unnecessary. Unfortunately, poor outcomes may result following a well indicated and well performed surgical procedure. It has also been shown that $20 \%$ to $30 \%$ of patients over 65 who underwent lumbar spine operations had one or more subsequent operations within four years (289). Waddell et al (290) noted that in all studies of back pain, $10 \%$ to $15 \%$ of patients account for $80 \%$ to $90 \%$ of the total health care compensation and cost for spinal disorders, and the $1 \%$ to $2 \%$ of patients who undergo surgery are the most expensive group. Keskimaki et al (292), in a study of population-based regional and interspeciality variations of lumbar disc surgery and reoperations described that back surgery in the United States has been shown to be five times more common than in the United 
Kingdom, three times more common than in Sweden, and two times more common than in Finland. They also noted up to 15-fold variations across regions of the United States. Multiple studies evaluating surgical treatment of lumbar disc prolapse and degenerative lumbar disc disease have shown conflicting evidence on the effectiveness of surgical discectomy for lumbar disc prolapse (290, 291, 293). Evidence is limited and contradictory for automated percutaneous discectomy (290), with no acceptable evidence on the effectiveness of any form of fusion for back pain or instability (290), no acceptable evidence on the efficacy of any form of decompression for degenerative lumbar disc disease or spinal stenosis (290), and no evidence as to whether any form of surgery for degenerative lumbar disc disease is effective in returning patients to work (290).

The sacroiliac joint, which receives its innervation from lumbosacral roots, is alleged to be a source of back pain or referred pain; and prevalence has been shown to be $19 \%$ to $30 \%$ in selected population groups $(191,192)$. The exact incidence of pain emanating from atlantoaxial and atlantooccipital, and thoracic facet joints is not known (294299). A multitude of other spinal conditions including, degenerative disorders and myofascial syndromes, contribute approximately to 5 to $10 \%$ of the spinal pain (163, 164, 171, 172, 186-190, 300-304).

Causes of nonspinal pain include the various causes responsible for headache; trigeminal neuralgia with facial pain; cancer pain with involvement of various musculoskeletal structures, either with the spread of the cancer into bones and muscles, with compression of the spinal cord, or pain after multiple surgical procedures radiotherapy or chemotherapy interventions; pain secondary to pressure on various nerve plexuses resulting in neuropathic pain; and, finally, pain resulting from visceral organs. Other causes include reflex sympathetic dystrophy and causalgia or complex regional pain syndromes Types I and II; postherpetic neuralgia, phantom limb pain; and finally, the controversial myofascial pain $(171,172,186-215)$. Even though some prevalence studies have been published occasionally, there are no controlled or systematic studies to show the prevalence of various disorders resulting in chronic pain.

\section{EVALUATION}

Appropriate history, physical examination, and medical decision making from the initial evaluation of a patient's presenting symptoms. A patient's evaluation should not only meet all the required medical criteria but also meet the regulatory requirements (305). The guidelines of the Health Care Financing Administration (HCFA) provide various criteria for five levels of services. The three crucial components of evaluation and management services are: history, physical examination, and medical decision making. Other components include: counseling, coordination of care, nature of presenting problem, and time. AHCPR Guidelines for managing acute low back problems in adults (28) also have provided guidance on initial clinical assessment, assessment of psychosocial factors, imaging techniques, and assessment with electromyography and nerve conduction. While there are numerous techniques to evaluate a chronic pain patient, variable from physician to physician and text book to text book, following the guidelines established by HCFA not only will assist a physician in performing a comprehensive and complete evaluation but also assist them to be in compliance with regulations.

\section{History}

The history includes:

$\quad$ Chief complaint,
History of present illness,
$\quad$ Review of systems, and,
$\quad$ Past, family, and/or social history.

Chief Complaint: The chief complaint is a concise statement describing the symptom, problem, condition, diagnosis, or other factor that is the reason for the encounter, usually stated in the patient's words.

History of Present Illness: The history of present illness is a chronological description of the development of the patient's present illness from the first sign and/or symptom. It includes the following elements:

$\begin{array}{ll} & \text { Location, } \\ & \text { Quality, } \\ & \text { Severity, } \\ & \text { Context, } \\ & \text { Modifying factors, and } \\ & \text { Associated signs and symptoms. }\end{array}$

Review of Systems: The review of systems is an inventory of body systems obtained through a series of questions seeking to identify signs and/or symptoms that the patient may be experiencing or has experienced.

Past, Family, and/or Social History: The past, family, 
and/or social history consists of a review of the past history of the patient including past experiences, illnesses, operations, injuries, and treatment; family history, including a review of medical events in the patient's family, hereditary diseases, and other factors; and social history appropriate for age reflecting past and current activities.

Past history in interventional pain medicine includes history of past pain problems, motor vehicle, occupational, or nonoccupational injuries; history of headache, neck pain, upper-extremity pain, pain in the upper, or mid back or chest wall, pain in the lower back or lower extremities, and pain in joints; and disorders such as arthritis, fibromyalgia, or systemic lupus erythematosus.

Family history includes history of pain problems in the family, degenerative disorders, familial disorders, drug dependency, alcoholism, or drug abuse; and psychological disorders such as depression, anxiety, schizophrenia, and suicidal tendencies, etc. Family history of medical problems is also important.

Social history includes environmental information, educa-

Table 4. Features of somatic and radicular pain

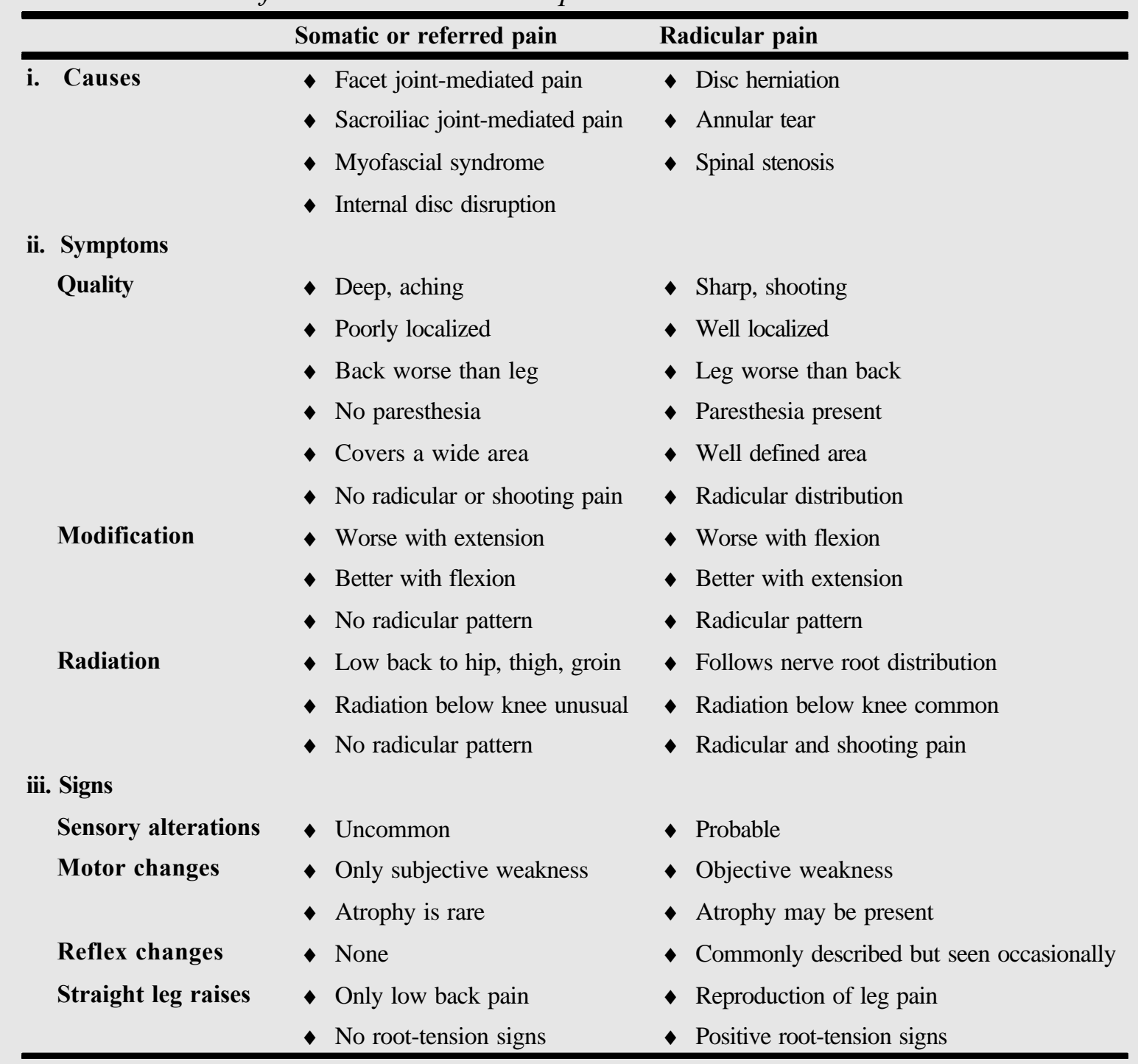

Adapted and modified from Manchikanti (41) 
tion, marital status, children, habits, hobbies, and occupational history, whenever available.

\section{Physical Examination}

Physical examination in interventional pain medicine involves general, musculoskeletal, and neurological examination.

Examination of other systems, specifically cardiovascular, lymphatic, skin, eyes, and cranial nerves is recommended based on the presenting symptomatology.

\section{Medical Decision Making}

Medical decision making refers to the complexity of establishing a diagnosis and/or selecting a management option as measured by three components, including;

1. Diagnosis/management options with a number of possible diagnoses and/or the number of management options;

2. Review of records/investigations, with number and/or complexity of medical records, diagnostic tests, and other information that must be obtained, reviewed, and analyzed; and ,

3. Risk(s) of significant complications, morbidity and mortality, as well as comorbidities associated with the patient's presenting problem(s), the diagnostic procedure(s), and/or the possible man- agementoptions.

Psychological evaluation, laboratory evaluation, imaging techniques, electromyography and nerve conduction and somatosensory evoked potentials are also an extension of evaluation process. It is beyond the scope of these guidelines to discuss these techniques of assessment.

Appropriate history and physical examination with the assistance of other evaluations should direct a physician to formulate a provisional diagnosis. Features of somatic and radicular pain are outlined in Table 4. However, various pitfalls with conventional evaluation of low back pain are also illustrated in Table 5. A suggested algorithm for comprehensive evaluation and management of chronic pain is illustrated in Fig. 2. In summary, the following criteria should be considered carefully in performing interventional techniques:

1. Complete initial evaluation, including history and physical examination.

2. Physiological and functional assessment, as necessary and feasible.

3. Definition of indications and medical necessity:

- $\quad$ Suspected organic problem.

- $\quad$ Nonresponsiveness to less invasive modalities of treatments except in acute situations such as acute disc herniation, herpes zoster and postherpetic neural-

Table 5. Pitfalls with conventional evaluation of low back pain

"Specific anatomic etiology is clearly and objectively identified in only $10 \%$ to $20 \%$."

1. Radiographic "abnormalities" are frequently clinically irrelevant.

2. True sciatica occurs in only $1 \%$ to $2 \%$ of the patients.

3. No universal criteria are established for scoring the presence, absence, or importance of particular signs.

4. Quantification of the degree of disability and the association to treatment outcomes is difficult.

5. Interpretation of biomedical findings relies on "clinical judgments," "physician's experience," and "quasi-standardized criteria."

6. Routine clinical assessment is frequently subjective and unreliable.

7. Physical examination and diagnostic findings are subjective.

8. The discriminative power of common objective signs has been questioned.

9. Reliance on general "clinical impression" to detect gross psychological disturbances is "hopelessly inaccurate."

10. It is usually not possible to make a precise diagnosis or identify anatomic origin of the pain by routine clinical assessment.

Adapted and modified from Manchikanti (41). 


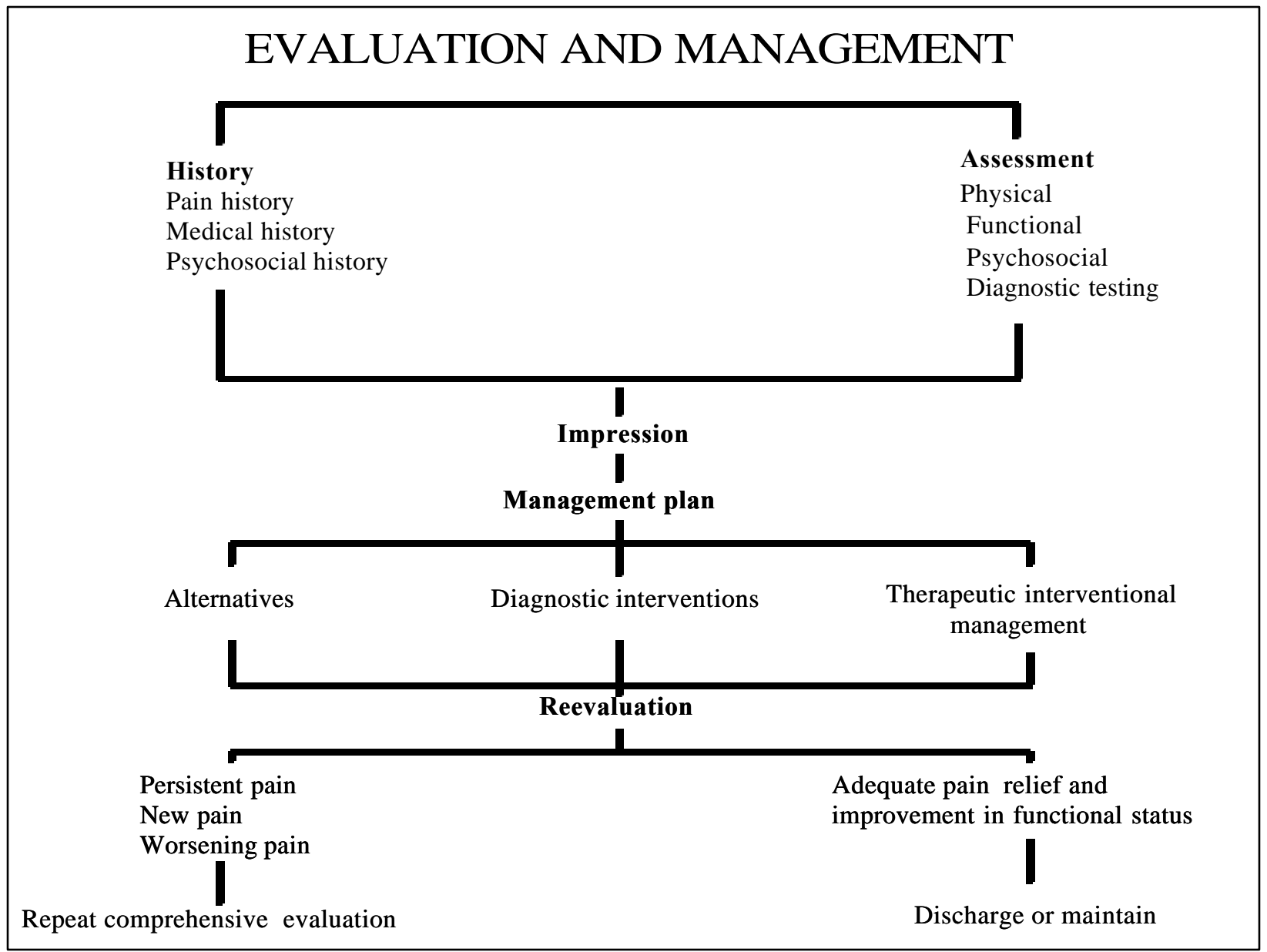

Fig. 2. Suggested algorithm for comprehensive evaluation and management of chronic pain

gia, reflex sympathetic dystrophy, and intractable pain secondary to carcinoma.

- $\quad$ Pain and disability of moderate-to-severe degree.

- $\quad$ No evidence of contraindications such as severe spinal stenosis resulting in intraspinal obstruction, infection, or predominantly psychogenic pain.

- $\quad$ Responsiveness to prior interventions with improvement in physical and functional status for repeat blocks or other interventions.

- $\quad$ Repeating interventions only upon return of pain and deterioration in functional status.

INTERVENTIONAL TECHNIQUES

\section{History}

The history of the application of interventional techniques in pain management dates back to 1901, when epidural injections for lumbar nerve root compression were reported (307-309). Since then, substantial advances have been made in the administration of epidural injections, and a multitude of other interventional techniques have been described (310-324). Thus, percutaneous injection techniques have been distinguished as the favored, and at times decisive, intervention in the diagnostic and therapeutic management of chronic painful conditions.

\section{Mechanism}

The overall benefit of various types of injection techniques includes pain relief outlasting by days, weeks, or months the relatively short duration of pharmacologic action of 
the local anesthetics and other agents used. Clear-cut explanations for these benefits are not currently available. It is believed that neural blockade alters or interrupts nociceptive input, reflex mechanisms of the afferent limb, self sustaining activity of the neuron pools and neuraxis, and the pattern of central neuronal activities (325). The explanations are based in part on the pharmacological and physical actions of local anesthetics, corticosteroids, and other agents. It is also believed that local anesthetics interrupt the pain-spasm cycle and reverberating nociceptor transmission, whereas corticosteroids reduce inflammation either by inhibiting the synthesis or release of a number of pro-inflammatory substances (326-332). Various modes of action of corticosteroids include membrane stabilization; inhibition of neural peptide synthesis or action; blockade of phospholipase $\mathrm{A}_{2}$ activity; prolonged suppression of ongoing neuronal discharge; suppression of sensitization of dorsal horn neurons; and reversible local anesthetic effect (327-340). In addition, local anesthetics have been shown to produce prolonged dampening of c-fiber activity (341-343). Physical effects include clearing adhesions or inflammatory exudates from the vicinity of the nerve root sleeve. The scientific basis of some of these concepts, at least in part, is proven for spinal pain management with epidural injections of betamethasone, and intravenous methylprednisolone (330, 334-337).

\section{DIAGNOSTIC INTERVENTIONAL TECHNIQUES}

Diagnostic blockade of a structure with a nerve supply, which can generate pain, can be performed to test the hypothesis that the target structure is a source of the patient's pain (32). Testing the hypothesis by provoking pain in any structure is an unreliable criterion except in provocative discography (175). However, neurodiagn-ostics of the involved nerve pathways has proven valuable. The relief of pain, however, is the essential criterion in almost all structures including analgesic discography in the cervical spine, the only deviation being lumbar discs (32). If the pain is not relieved, the source may be in another structural component of the spine similar to the one tested such as a different facet joint or a different nerve root or some other structure (32). Thus, precision diagnostic injections directed towards specific spinal pathology are potentially powerful tools for diagnosis of chronic spinal pain, but often technically challenging. Identifying the specific pathology responsible for pain is often difficult, leading to frustrated patients and clinicians. Nevertheless, these injections may be safely performed by properly trained anesthesiologists, physiatrists, neurologists, radiologists, spine surgeons and physicians from other related specialties who take the time to learn the basis for and perfect the application of these techniques.

When the source of pain is more than one structure or multiple levels, it is not expected that all the pain will be relieved. For example, there may be painful facet joints bilaterally at a given segmental level, in which case anesthetizing the left joint should relieve the left side, but not the right side; there may be pain from two consecutive joints on one side, in which case anesthetizing the lower joint alone may relieve only the lower half of the pain; there may be more than one structure involved, such as pain contributed by discs and facet joints or facet joints and nerves (32).

True positive responses are secured by performing controlled blocks. Ideally, this should be in the form of placebo injections of normal saline; but logistical and/or ethical considerations prohibit the use of normal saline in conventional practice.

\section{Rationale}

The rationale for diagnostic neural blockade in the management of spinal pain stems from the fact that clinical features and imaging or neurophysiologic studies do not permit the accurate diagnosis of the causation of spinal pain in the majority of patients in the absence of disc herniation and neurological deficit (28-30, 32, 33, 41, 42, 56, $58,62,151-153,162-164,174-185,306,344-357)$. It was also shown that sacroiliac joint pain is resistant to identification by the historical and physical examination data (166, $167,191,192,358-360)$, even though some have claimed sensitivity in the range of $60 \%$ to $87 \%$ with multiple provocative maneuvers $(361,362)$. In addition, no corroborative radiologic findings have been identified in patients with sacroiliac joint syndrome (363-371). Further rationale is based on the recurring facts showing the overall rate of inaccurate or incomplete diagnosis in patients referred to pain treatment centers to range from $40 \%$ to $67 \%$, the incidence of psychogenic pain to be only 1 in 3,000 patients, and the presence of organic origin of the pain is mistakenly branded as psychosomatic in $98 \%$ of the cases $(144,145)$. Finally, the most compelling reason is that chronic low back pain is a diagnostic dilemma in $85 \%$ of patients even in experienced hands with all the available technology (Table 5). It has been determined that utilizing alternative means of diagnosis including precision diagnostic blocks in cases where there is a lack of definitive diagnostic radiologic or electrophysiologic criteria can 
enable an examiner to identify the source of pain in the majority of patients, thus reducing the proportion of patients who cannot be given a definite diagnosis from $85 \%$ to $35 \%$ or even as low as $15 \%$.

\section{Facet Joint Blocks}

The facet joints of the spine can be anesthetized by fluoroscopically guided injections of local anesthetic, either into the target joint or onto the medial branches of the dorsal rami that supply them $(32,33,41,163,178-185,372-380)$.

The rationale for facet joint blocks is based on the observation that if a particular joint is determined to be the source of pain generation, long-term relief can be sought by directing therapeutic interventions at that joint. In managing low back pain, local anesthetic injection into the facet joints or interruption of the nerve supply to the facet joints has been accepted as the standard for diagnosis of facet joint mediated pain. Since a single joint is innervated by at least two medial branches, two adjacent levels should always be blocked.

Instead of placebo-controlled diagnostic facet joint blocks, a convenient control is the use of comparative local anesthetic blocks, in which on two separate occasions the same structure is anesthetized, but using local anesthetic with different durations of action. However, one of the drawbacks of local anesthetic control is that comparative local anesthetic blocks may not be implementable for intra-articular blocks because it is not known whether the placement of local anesthetic in a relatively avascular environment such as a joint space affects its expected duration of action, and leakage of local anesthetic from the joint capsule onto the exiting nerve root may give a false positive response. On the contrary, these are implemented readily for medial branch blocks and probably for other types of nerve blocks. With medial branch blocks, the use of comparative local anesthetic blocks has been evaluated and found to be valid against challenge with placebo $(32,372$ 374).

A diagnosis cannot be rendered reliably on the basis of a single block because false-positive rates are seen in as many as $41 \%$ of patients $(32,178-185,372-374)$. Hence, controlled blocks with comparative local anesthetics are required in essentially every case (32). Even then, comparative blocks are only $85 \%$ reliable.

\section{Discography}

Once stifled by misinformation, discography now has applications in a number of clinical settings (173, 381-404). The first to create widespread interest in the disc as a source of pain was Mixter and Barr with their 1934 hallmark description of the herniated nucleus pulposus (222). This mechanical model detailed a lumbar posterolateral prolapse with direct nerve root compression and secondary radiculopathy. The work of Mixter and Barr (222) became the central model of spine pain, which preoccupied the medical community and diverted attention from other possible causes, even though Mixter and Ayers in 1935 demonstrated that radicular pain can occur without disk herniation.

Formal studies in normal volunteers have shown that lumbar disc stimulation provocative discography is a specific test as lumbar discs are presumed not to hurt in asymptomatic individuals $(381,392)$. Thus, finding a painful disc in a patient is considered as a significant observation. However, even so, controls are mandatory to exclude false-positive responses to refute the competing hypothesis that stimulating any disc reproduces the patient's pain (381, 393-402). The IASP has recommended that for disc stimulation to be considered valid, at least one, and preferably two, adjacent discs be stimulated as controls. Hence, for a disc to be deemed painful, stimulation of that disc, but neither of the adjacent discs, should reproduce the patient's pain. In contrast to lumbar discs, in the evaluation of cervical discogenic pain provocative cervical discography or cervical disc stimulation is not as well documented as the lumbar spine. It is not clear that cervical discs do not hurt in normal volunteers to the same extent as lumbar discs.

In 1988, the North American Spine Society (NASS) published a position statement about discography (395). Discograms were considered a procedure only for those with chronic low back pain (symptoms greater than 4 months duration). The document recognized that other than discography, no visualization tool offers the ability to precisely delineate disk morphology (396). The NASS updated its position paper through its diagnostic and therapeutic committee in 1995 (397). According to the position statement on discography by the NASS $(395,397)$ :

Discography is indicated in the evaluation of patients with unremitting spinal pain, with or without extremity pain, of greater than four months' duration, when the pain has been unresponsive to all appropriate methods of conservative 
therapy. Before discography, the patients should have undergone investigation with other modalities which have failed to explain the source of pain; such modalities should include, but not be limited to, either computed tomography (CT) scanning, magnetic resonance imaging (MRI) scanning and/or myelography. In these circumstances, discography, especially when followed by CT scanning, may be the only study capable of providing a diagnosis or permitting a precise description of the internal anatomy of a disc and a detailed determination of the integrity of the disc substructures. Additionally, the anatomic observations may be complemented by the critical physiological induction of pain, which is recognized by the patient as similar to or identical with his/her complaint. By including multiple levels in the study, the patient acts as his/ her own control for evaluation of the reliability of the pain response.

Other indications for discography include: (1) ruling out secondary internal disc disruption or recurrent herniation in the postoperative patient; (2) exploring pseudoarthrosis; (3) determining the number of levels to include in a spine fusion; and (4) identifying the primary symptom-producing level when chemonucleolysis (enzymatic hydrolysis) or anular denervation (via thermocoagulation with an intradiscal catheter or a radiofrequency probe) is contemplated $(173,226)$.

There are several potential sources of both false-positive and false-negative responses with provocative discography. Carragee et al $(398,399)$ concluded, that in individuals with normal psychometrics and without chronic pain, the rate of false-positives is very low if strict criteria are applied; and that the false-positive rate increases with abnormal psychometrics and increased annular disruption. Carragee et al (400) also showed that a high percentage of asymptomatic patients (40\%) with normal psychometric testing who previously have undergone lumbar discectomy will have significant pain on injection of their discs that had previous surgery. Carragee et al (401) showed that even though a high-intensity zone is seen more commonly in symptomatic patients, the prevalence of a high-intensity zone in asymptomatic individuals with degenerative disc disease also was too high $(25 \%)$ for meaningful clinical use. Carragee et al (402) also showed that discography does not cause long term back symptoms in previously asymptomatic subjects with normal psychometrics.

\section{Selective Epidural Injections}

As in the case with the intervertebral disc, spinal nerves can be injected with contrast, local anesthetic, or other substances (353). Both the provocative response and analgesic response provide clinically useful information. Steindler and Luck (318) recognized the validity of provocative and analgesic spinal injections as early as 1938 . In 1971, McNab and coworkers (405) revealed the value of diagnostic, selective nerve root blocks in the preoperative evaluation of patients with negative imaging studies and clinical findings of root irritation. The nerve blocks were utilized to diagnose the source of radicular pain when imaging studies suggested possible compression of several nerve roots (406-418). The relief of usual symptoms following the injection of local anesthetic, $1 \mathrm{~mL}$ of $2 \%$ Xylocaine, was the main determinant for diagnostic information. Schutz and colleagues (407), Krempen and Smith (408), Tajima and colleagues (409), Haueisen and coworkers (410), Dooley and colleagues (411), and Stanley and coworkers (412) described positive results of diagnostic selective nerve root blocks. In 1992, Nachemson (419) analyzed the literature on low back pain and indicated that diagnostic, selective nerve root block provided important prognostic information about surgical outcome.

Kikuchi and colleagues (415) estimated that approximately $20 \%$ of the patients presenting with apparent radicular pain required diagnostic nerve root blocks or epidural blocks. Van Akkerveeken (420) recreated data from his 1989 thesis regarding sensitivity, specificity, and predicative values for diagnostic, selective nerve root blocks. A positive block required concurrent symptom reproduction during root stimulation and full relief following anesthetic infusion (416). Derby et al (413) correlated surgical outcome with pain relief following transforaminal epidural injections with local anesthetic and steroids and reported that patients who failed to obtain sustained relief of radicular pain following the block were less likely to benefit from subsequent surgical intervention.

The controversial aspects of epidural injections include the terminology and technique (58). The terminology describing nerve root injections has varied from transforaminal epidural to selective nerve root block, selective nerve root sleeve injection, selective epidural, selective spinal nerve block, or selective ventral ramus block. However, 


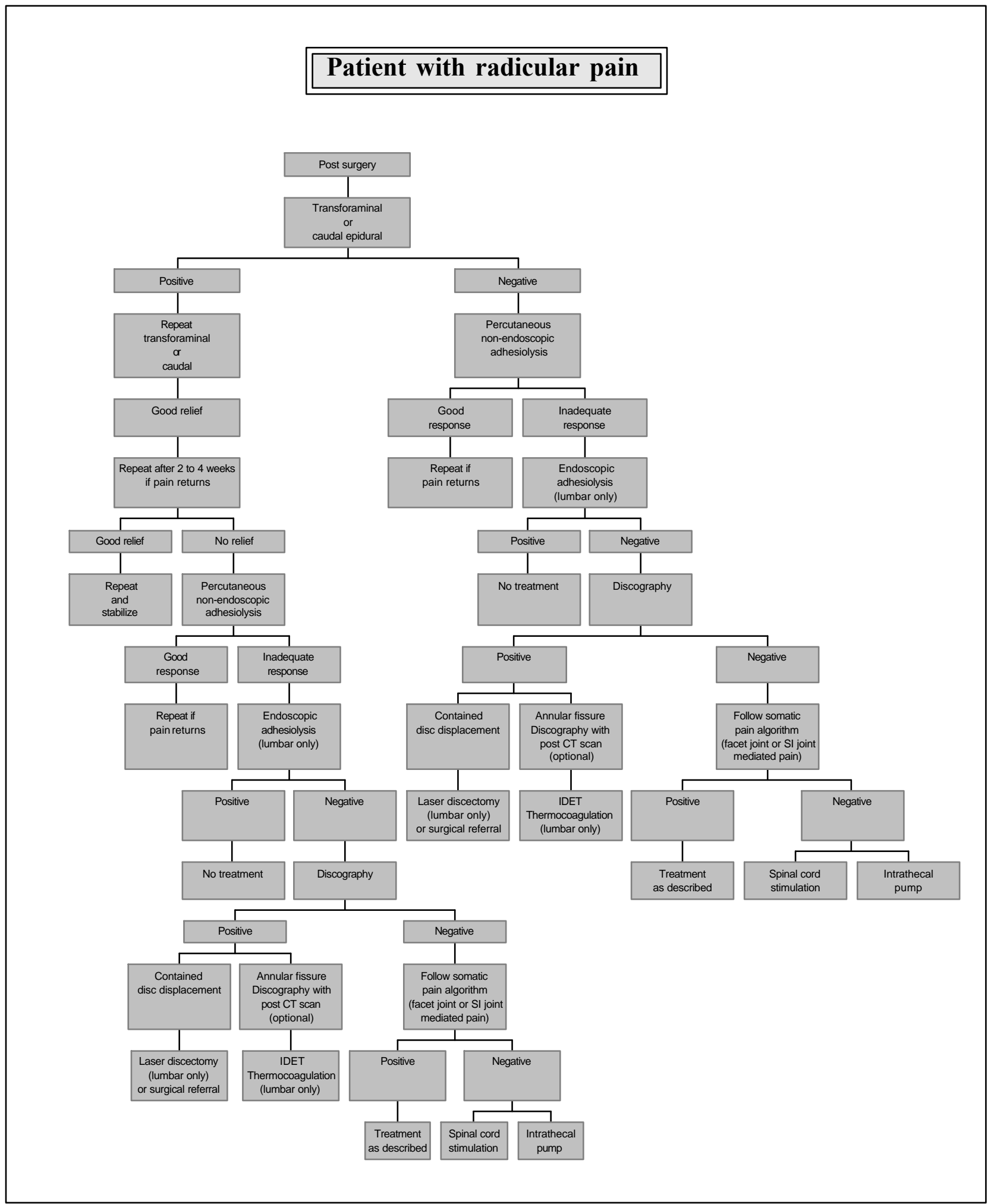

Fig. 3A. A suggested algorithm for application of interventional techniques in conservative care of chronic spinal pain: A patient with radicular pain 


\section{Patient with radicular pain}

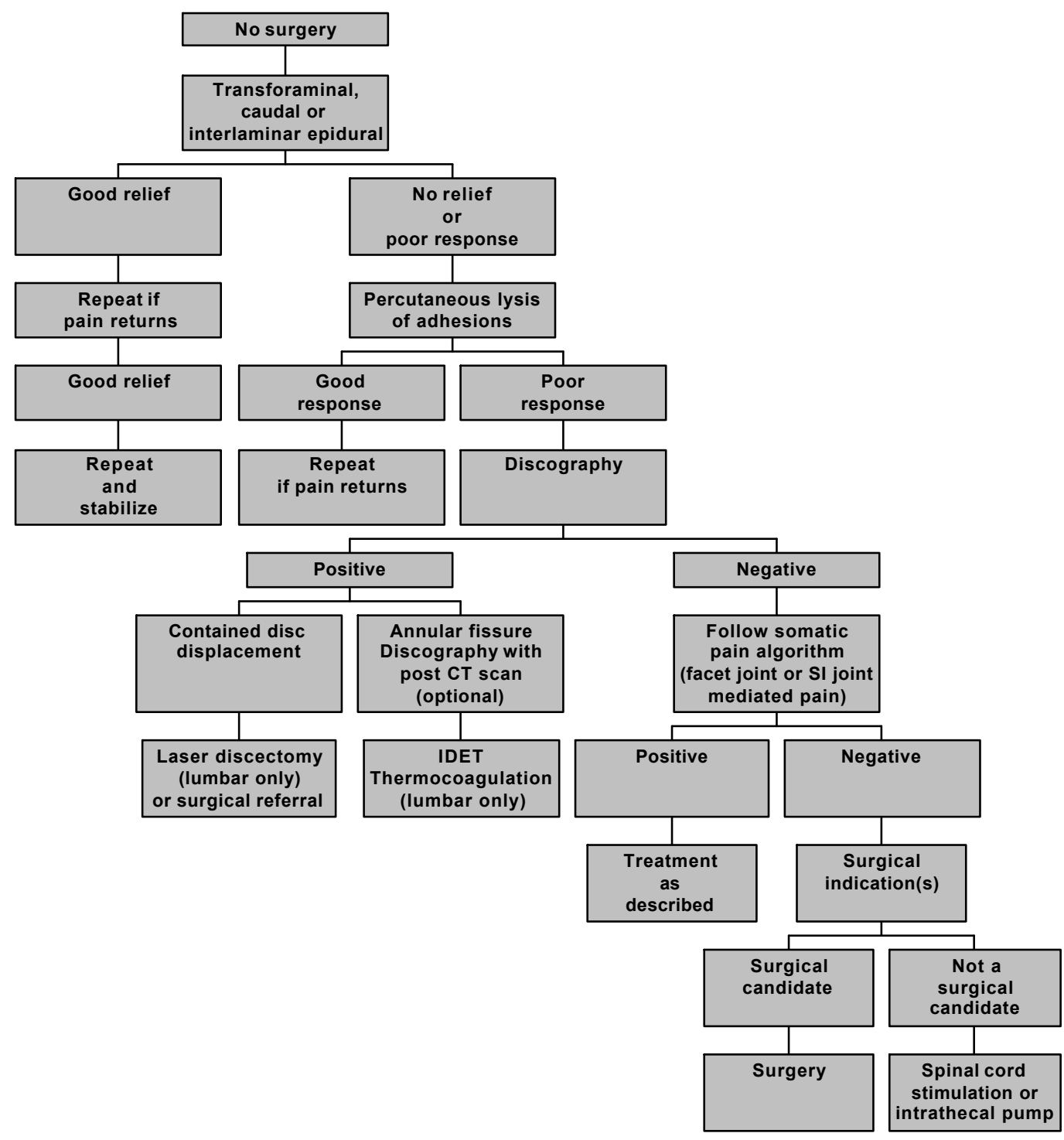

Fig. 3B. A suggested algorithm for application of interventional techniques in conservative care of chronic spinal pain: A patient with radicular pain 


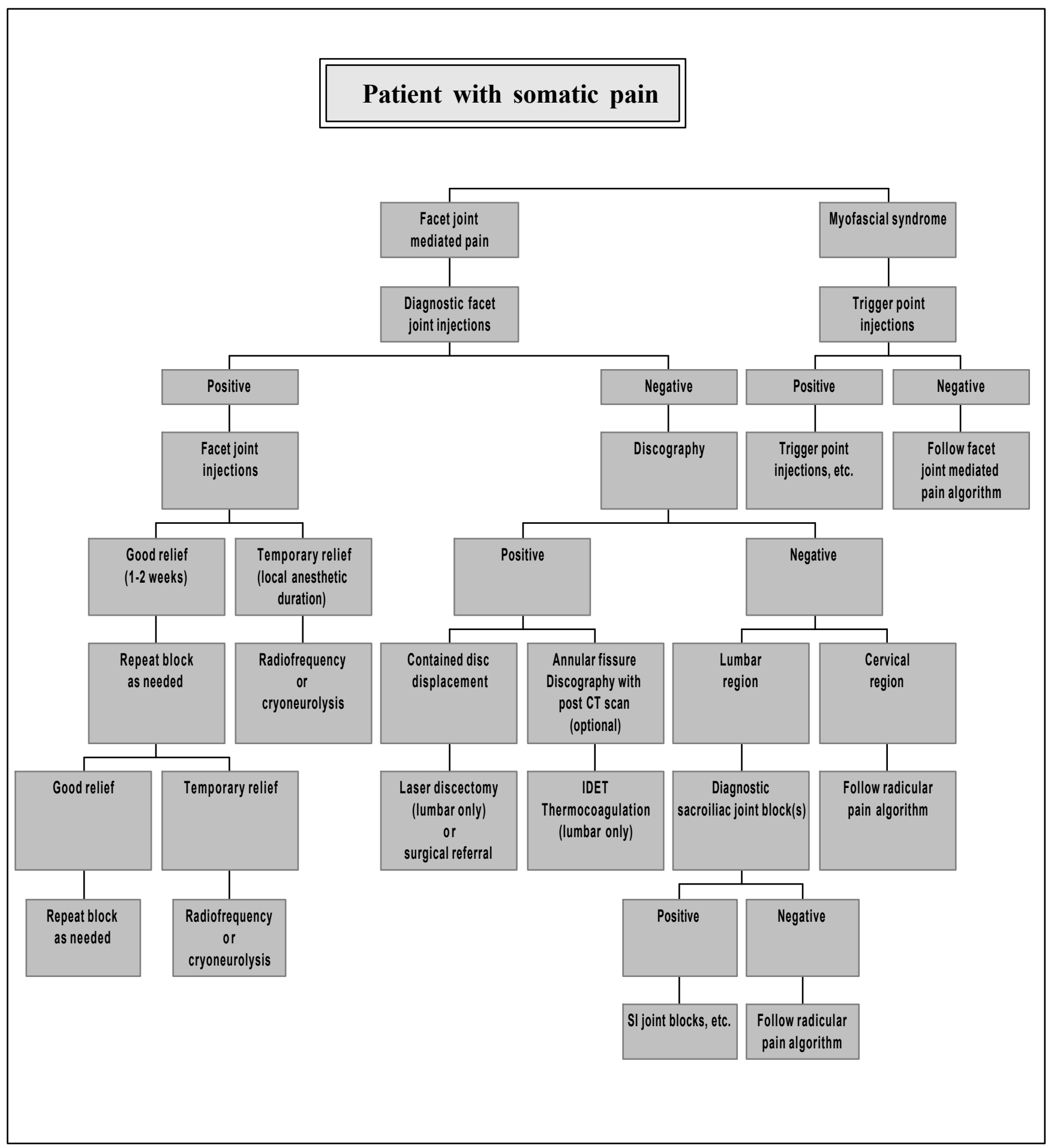

Fig. 4A. A suggested algorithm for application of interventional techniques in conservative care of chronic spinal pain: A patient with somatic pain 


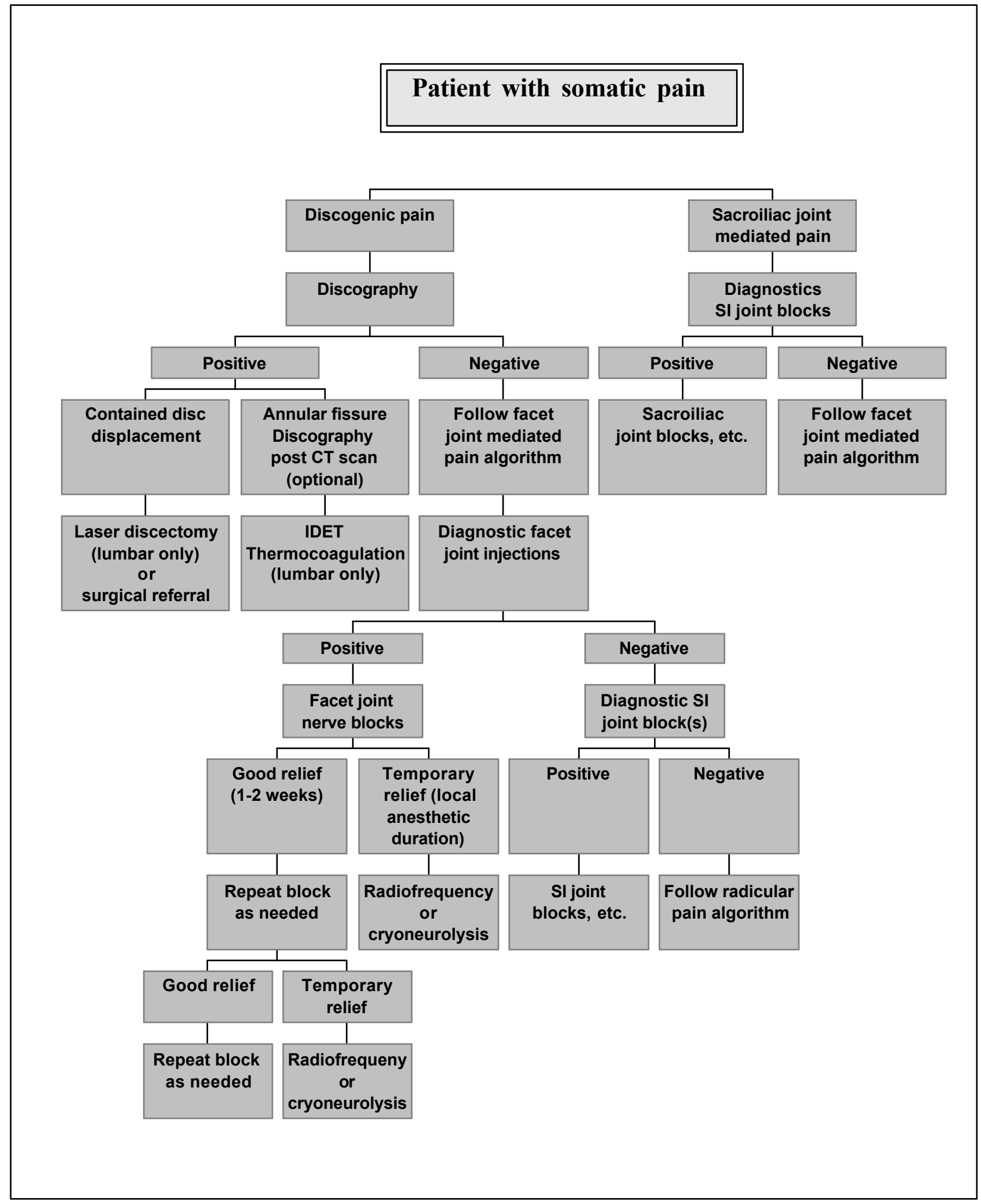

Fig. 4B. A suggested algorithm for application of interventional techniques in conservative care of chronic spinal pain: A patient with somatic pain 
nerve root block was the first term developed to describe the technique for diagnosing the source of radicular pain when imaging studies suggested a possible compression of several roots. Early studies of selective nerve root injections described an extra-foraminal approach, in which the needle is advanced at a right angle to the spinal nerve outside the neural foramina. Subsequently, a variation of this procedure has emerged which has been termed selective epidural and is also referred to as transforaminal epidural.

\section{Sacroiliac Joint Injections}

Sacroiliac joint has regained interest as a primary source of low back pain in recent years, but confirming the diagnosis of sacroiliac joint dysfunction and pain remains difficult. Even though intra-articular sacroiliac joint injections have provided information on pain referral patterns (166, 167, 359), detecting symptomatic joints in patients presenting with low back pain continues to be a difficult venture $(358,360-371)$. Thus, provocative injections remain the only direct method to distinguish symptomatic from asymptomatic joints. Schwarzer et al (191), utilizing single local anesthetic block reported a prevalence of $30 \%$ in chronic low back pain population. Maigne et al (192), utilizing a double block paradigm with comparative local anesthetics reported prevalence in chronic low back pain population of $19 \%$ with a false-positive rate of $29 \%$.

\section{An Algorithmic Approach}

Two suggested algorithms for the application of interventional techniques in conservative care of chronic spinal pain describing steps for diagnosis and management are shown in Fig. 3 and 4. These are only suggested algorithms and are limited to the management of chronic spinal pain. Further, clinical evaluation in spite of drawbacks is extremely important, as is the documentation of indications for interventional techniques.

The clinical algorithms presented on the following pages show an effort to blend conscientious, explicit, and judicious use of the current best evidence in making decisions about the care of individual patients. When this is combined with the clinician's experience and judgment, and patient preferences, it should result in improved outcomes and significantly improved quality of care. These guidelines are intended to establish a boundary of reasonable care giving latitude to the individual physician.

\section{THERAPEUTIC INTERVENTIONAL}

\section{TECHNIQUES}

\section{Rationale}

The rationale for therapeutic interventional techniques in the spine is based upon several considerations: the cardinal source of chronic spinal pain, namely discs and joints, are accessible to neural blockade; removal or correction of structural abnormalities of the spine may fail to cure and may even worsen painful conditions; degenerative processes of the spine and the origin of spinal pain are complex; and the effectiveness of a large variety of therapeutic interventions in managing chronic spinal pain has not been demonstrated conclusively (27-32, 66-69, 261-291, 421456). Tulder et al (421) evaluated conservative treatment of chronic low back pain and studied the evidence for effectiveness of numerous conservative modalities used in managing chronic low back pain, including drug therapy, manipulation, back schools, electromyographic biofeedback therapy, exercise therapy, traction and orthoses, behavioral/cognitive/relaxation therapy, and transcutaneous electrical nerve stimulation. Overall results were highly variable for various conservative modalities of treatment in managing chronic low back pain. They have not studied either the differences between various types of epidural steroid injections, or lysis of adhesions. In addition, they also omitted facet joint injections, facet joint nerve blocks, and medial branch neurotomy. Similarly, surgical treatment of lumbar disc prolapse and degenerative lumbar disc disease was also without conclusive evidence (290). There are a multitude of interventional techniques in the management of chronic pain which include not only neural blockade but also minimally invasive surgical procedures ranging from peripheral nerve blocks, trigger-point injections, epidural injections, facet joint injections, sympathetic blocks, neuroablation techniques, intradiscal thermal therapy, disc decompression, morphine pump implantation, and spinal cord stimulation.

In developing these guidelines, we have evaluated the effectiveness of the most common interventional therapeutic interventions for chronic pain in general, and specifically chronic spinal pain. Koes et al (66) concluded that the methodological quality of clinical trials of the efficacy of the commonly used interventions in low back pain was disappointingly low. For these guidelines, a modest approach including a blend of scientific evidence together with expertise and consensus was utilized. All the trials were scored according to the criteria described (45).

Whenever applicable, we used the original scores of pre- 
viously published systematic reviews $(45,66,67,421)$. A study was considered positive if the therapeutic intervention was more effective than the reference treatment with regard to at least one of the outcome measures, which included pain intensity, overall improvement, functional status, and return to work. The level of evidence was also a blend of evidence from AHCPR guidelines (28), as well as evidence based guidelines from McQuay and Moore (7). The blended rating system consisted of five levels of evidence based on the strength as shown in Table 1 .

\section{Facet Joint Mediated Pain}

A preponderance of evidence supports the existence of lumbar facet joint pain $(31,32,41,56,152,154-157,177-$ $183,374,376-380,457-472)$; however, there are also a few detractors $(348,351,473,474)$. The diagnosis of the so called lumbar facet syndrome depends on a clinical presentation with mechanical low back pain described by the patient as mainly in the low back with radiation to the buttocks and upper posterior thigh. Some investigators have attempted to identify facet syndrome and predictors of outcome of facet joint injections, which has been rather futile. The results of most studies failed to show a correlation between radiologic imaging findings, clinical examination, and the controlled diagnostic blocks (183). However, the features of somatic pain may be utilized as a guide presumably to differentiate somatic pain and radicular pain, at least initially (Table 4). Similarly, there is also a preponderance of evidence supporting the existence of cervical facet joint pain $(31,32,42,158-164,184,185,475-$ 480). Interestingly, the controversy appears to be less in the cervical spine than in the lumbar spine.

Facet joint mediated pain may be managed by either intraarticular injections, medical branch blocks, or neurolysis of medial branches.

Intra-articular Injections: Therapeutic benefit has been reported with the injection of corticosteroids $(458,460$, $462,463,470)$, local anesthetics $(457,474)$, or normal saline $(347,457,468)$ into the facet joints. The literature describing the effectiveness of these interventions is abundant, however, only six randomized clinical trials offer data on the use of intra-articular injections in the spine (379, 380, 457, 468, 469, 475). Open, uncontrolled clinical studies, which evaluated the long term relief of back and leg pain from intra-articular facet joint injections reported variable relief in $18 \%$ to $92 \%$ of subjects (458-460, 464-467, $470,471,476-480$ ). Five studies of intra-articular corticosteroid lumbar facet joint injections, and one study in cervical spine were performed comparing the results to those of a similar group not receiving intra-articular steroids (Table 6).

In a prospective, controlled study Carette et al (457) studied 101 patients who received more than 50\% relief with a single intra-articular lidocaine block. Those patients who responded were randomized into two treatment groups: intra-articular saline or intra-articular methylprednisolone. At 1-month follow-up after the injection, $42 \%$ of the methylprednisolone group (20 patients) had significant pain reduction, whereas $33 \%$ of the saline group (16 patients) achieved significant pain relief. At 6-month follow-up, however, $46 \%$ of the patients in the methylprednisolone group and $15 \%$ of patients in the saline group continued to experience marked pain relief, with a statistically significant difference.

Lilius et al (468) studied 109 patients with chronic, unilateral, nonradicular low back pain who had failed to respond to conservative treatment, including medication and physical therapy for a period of 3 to 36 months. A total of 27 of the 109 patients were postsurgical and had continued pain despite previous discectomy. They were randomly divided into three treatment groups: 1) intra-articular lumbar facet joint injection with cortisone and local anesthetic; 2) intra-articular injection with saline alone; or 3) pericapsular injection of cortisone and local anesthetic. Significant pain relief was reported by patients in all groups for up to three months. A total of $64 \%$ of the patients showed relief one hour after injection, and $36 \%$ of these patients reported relief from pain over a 3-month period, independent of the treatment given.

Lynch and Taylor (469) in a controlled, prospective (but not randomized or blinded) study, reported effectiveness of intra-articular placement of the corticosteroid without anesthetic in 50 patients. Extra-articular injection was used for patients in the control group. Total pain relief was reported in 9 of 27 patients who received intra-articular corticosteroids compared with none of the 15 patients who received extra-articular corticosteroids. Only two patients in the intra-articular group did not obtain at least partial benefit, whereas 7 of the 15 control patients had no relief at all.

Marks et al (380) compared the effects of intra-articular anesthetic and corticosteroid with medial branch blocks in a study of 86 patients with chronic low back pain. Patients were randomized and assigned to either facet joint injections or medial branch blocks using methylprednisolone 
acetate, $20 \mathrm{mg}$, and lidocaine, $1.5 \mathrm{~mL}, 1 \%$ at each level. They concluded that "Facet joint injections and facet nerve blocks may be of equal value as diagnostic tests, but neither is a satisfactory treatment for chronic low back pain."

Nash (379) compared facet joint injections with medial branch blocks in a randomized study of 67 patients in two treatment groups and a 1-month follow up period. No appreciable difference was evident in evaluations between the groups at follow-up.

Barnsley et al (475) studied 41 patients with neck pain caused by whiplash injury in a randomized, double-blinded investigation with a therapeutic trial of cervical intra-articular local anesthetic, or local anesthetic with steroid. Results from this study indicate that the time to return to $50 \%$ of baseline pain was three days in the steroid group and 3.5 days in the local anesthetic group. Less than half of the patients reported relief of pain for more than one week, and fewer than one in five patients reported relief for more than one month, regardless of whether injection was with steroids or local anesthetic. They (475) concluded that intra-articular injection of steroid was not an effective treatment for cervical facet joint pain associated with whiplash injuries. They cautioned that these results should not be extrapolated to the treatment of patients with cervical facet joint pain from other causes, because response to intra-articular steroid injections is not known in cervical facet joint pain of spontaneous origin.

However, all of the controlled studies summarized faced substantial criticism. Lilius et al (468) used overly broad inclusion criteria of patients with neurologic deficits, and the patient's diagnosis of lumbar facet joint mediated pain was not confirmed by the diagnostic blocks; furthermore, excessive volumes, ranging from $3 \mathrm{~mL}$ to $8 \mathrm{~mL}$ of active agents, were injected, and placebo responders were not excluded. Although the study by Carette et al (457) was praised for its design, these authors failed to exclude placebo responders, which may account for the relatively high incidence of patients in their study with presumed facet joint pain. Failure to exclude the placebo responders invariably dilutes the findings of true responses, making detection of difference between the study and control groups more difficult. Additional criticism against Carette's study (457) was that intra-articular lumbar facet joint corticosteroids were evaluated in isolation and not as part of a comprehensive, conservative treatment plan provided equally to both groups (472). Lack of randomization, poor outcome assessment tools, failure to select patients with isolated facet joint pain as determined by diagnostic blocks, and lack of third party review were among the weaknesses of the study by Lynch and Taylor (469). Marks et al study (380) is limited by failure to select patients with facet joint pain established by controlled diagnostic blocks; failure to have a blinded, independent observer; poor limited outcome assessment tools; and absence of a control or placebo group. Nash's study (379) is limited by lack of established diagnosis or confirmation of facet joint mediated pain; lack of a blinded observer; poor assessment tools; and lack of a controlled or placebo group. Barnsley et al (475) included a small number of patients (20 in each group), whose origin of neck pain was post traumatic, following whiplash.

Due to negative results of intra-articular injections, additional studies of observational nature with good-quality data were considered (Table 6). Of the multiple studies available on managing facet joint mediated pain, only six met the criteria for inclusion as observational studies with at least minimum of 50 patients and a reasonable follow up. Of these, four were prospective, including Jackson et al (351), Desoutet et al (460), Murtagh (465), Mironer and Somerville (471). The observational studies, which were of a retrospective nature, included Lippitt (462) and Lau et al (463). Jackson et al (351) prospectively evaluated 454 patients from 2,500 patients, with 390 patients completing the study. Even though this was a prospective study, there was no long term follow-up. Immediate relief was seen in only $29 \%$ of the patients. Desoutet et al (460) studied 54 patients, with immediate relief noted in $54 \%$ of the patients whom they considered as facet syndrome. Of the $54 \%$ of the patients diagnosed with facet syndrome by local anesthetic blocks, they reported $62 \%$ of the patients experiencing relief for 1 to 3 months, whereas $38 \%$ of the patients experienced relief for 6 to 12 months. Murtagh (465) studied 100 patients with a follow-up of up to 4 years reporting immediate relief in $94 \%$ of the patients and long term relief up to 6 months in $54 \%$ of the patients. Mironer and Somerville (471) evaluated 148 patients, injecting the facet joints with bupivacaine and steroid reporting $28 \%$ of patients obtained greater than $60 \%$ relief with a duration longer than two months. They also reported that these patients were observed and that eight patients required reinjection on an average of 4.8 months later with similar good results; thus, $28 \%$ of the patients, with or without repeat injections, reported relief up to 15 months at follow-up. Among the retrospective studies, Lau et al (463) reported the results in 50 patients with a follow-up period of 4 months to 18 months with initial relief in $56 \%$ of the patients, $44 \%$ at 3 months, and $35 \%$ at 6 to 12 months. Lippitt (462) reported results in 99 patients with a 12-month 


\begin{tabular}{|c|c|c|c|c|c|c|c|}
\hline \multirow[t]{2}{*}{ Study } & \multirow[t]{2}{*}{$\begin{array}{c}\text { Study } \\
\text { Characteristics }\end{array}$} & \multirow[t]{2}{*}{$\begin{array}{l}\text { No. of } \\
\text { Patients }\end{array}$} & \multirow[t]{2}{*}{$\begin{array}{l}\text { Drugs } \\
\text { Utilized }\end{array}$} & \multirow{2}{*}{$\begin{array}{c}\text { Initial Relief } \\
\text { 1-4 weeks } \\
\text { Controls vs } \\
\text { Treatment }\end{array}$} & \multicolumn{2}{|c|}{$\begin{array}{l}\text { Long-term Relief } \\
\text { Control vs Treatment }\end{array}$} & \multirow[t]{2}{*}{ Results } \\
\hline & & & & & 3 Months & 6 Months & \\
\hline \multicolumn{8}{|l|}{ Controlled Studies } \\
\hline Carette et al (457) & $\mathrm{P}, \mathrm{PC}, \mathrm{RA}$ & 101 & NS, LA, S & $33 \%$ vs $42 \%$ & N/A & $15 \%$ vs $46 \%$ & $N$ \\
\hline Barnsley et al (475) & $\mathrm{P}, \mathrm{RA}$ & 41 & LA, S & $50 \%$ & N/A & N/A & $N$ \\
\hline Lynch and Taylor (469) & $\mathrm{P}, \mathrm{C}$ & 50 & LA, S & $50 \%$ vs $92 \%$ & $62 \%$ & $56 \%$ & $P$ \\
\hline Lilius (468) & $\mathrm{P}, \mathrm{PC}, \mathrm{RA}$ & 109 & NS, LA, S & N/A & $64 \%$ & N/A & $N$ \\
\hline Nash (379) & $\mathrm{P}, \mathrm{RA}$ & 66 & LA, S & $58 \%$ & N/A & N/A & $N$ \\
\hline Marks et al (380) & $\mathrm{P}, \mathrm{RA}$ & 86 & LA, S & $45 \%$ & $18 \%$ & N/A & $N$ \\
\hline \multicolumn{8}{|l|}{ Observational Studies } \\
\hline Jackson et al (351) & $\mathrm{P}$ & 390 & LA, $\mathrm{S}$ & $29 \%$ & N/A & N/A & $N$ \\
\hline Murtagh (465) & $P$ & 100 & LA, S & $94 \%$ & $54 \%$ & $54 \%$ & $P$ \\
\hline Lippit (462) & $\mathrm{R}$ & 99 & LA, S & $51 \%$ & $51 \%$ & $14 \%$ & $P$ \\
\hline Lau et al (463) & $\mathrm{R}$ & 50 & LA, S & $56 \%$ & $44 \%$ & $35 \%$ & $P$ \\
\hline Desoutet et al (460) & $\mathrm{P}$ & 54 & LA, S & $62 \%$ & $38 \%$ & $38 \%$ & $P$ \\
\hline Mironer and Somerville (471) & $\mathrm{P}$ & 148 & LA, S & $28 \%$ & $28 \%$ & $28 \%$ & $N$ \\
\hline
\end{tabular}

$\mathrm{P}=$ prospective $; \mathrm{RA}=$ randomized $\mathrm{C}=$ controlled $\mathrm{PC}=$ placebo controlled $\mathrm{R}=$ retrospective $\mathrm{LA}=$ local anesthetic $; \mathrm{NS}=$ normal saline; $\mathrm{S}=$ steroids; $\mathrm{N} / \mathrm{A}=$ not available $; \mathrm{VS}=$ versus $; P=$ positive $;=$ negative

follow-up period with greater than $50 \%$ relief in $51 \%$ of the patients, which declined to $14 \%$ at 6 months and $8 \%$ at 12 months.

Of the six controlled studies, one was considered as of high quality (457), one as of moderate quality (475), and the remaining four as of low quality $(379,380,468,469)$. The results were positive in only one study (469). Observational evidence was positive with four of the six studies showing positive results. Based on the available evidence, both from randomized, controlled trials and observational studies, type and strength of efficacy evidence for intraarticular injections of facet joints is level III to IV - moderate to limited. Level III - moderate evidence is defined as evidence obtained from well-designed trials without randomization, single group pre-post, cohort, time series, or matched case controlled studies. Level IV - limited evidence is defined as evidence obtained from well-designed non-experimental studies from more than one center or research group.

Medial Branch Blocks: The role of medial branch blocks in the diagnosis of facet joint pain has been well described and superior to intra-articular comparative local anesthetic blocks $(31,32,56,161,162,178-185,372-380)$ even though controversy continues to exist (471). The therapeutic role of medial branch blocks with various adjuvants was evaluated only in one prospective randomized clinical trial (481). However, an additional three studies, which are controlled and randomized evaluated the role of initial blockade with its therapeutic effect $(182,379,380)$. In addition, two uncontrolled studies evaluated the medial branch blocks with respect to long term relief $(375,377)$ (Table 7).

Manchikanti et al (481) studied patients who had a diagnosis of facet joint mediated pain confirmed by controlled diagnostic blocks. These patients were randomly allocated into two groups, either receiving therapeutic medial branch blocks with a local anesthetic and Sarapin $®$ or receiving therapeutic medial branch blocks with a mixture of local anesthetic, Sarapin, and methylprednisolone. A total of 73 patients were enrolled in the study with ability to per- 
form at least two injections. The injections consisted of medial branch blocks with a mixture of local anesthetic 0.5 to $1 \mathrm{~mL}$ mixed with equal volumes of Sarapin in group I, with addition of $1 \mathrm{mg}$ of methylprednisolone per $\mathrm{mL}$ to the mixture in group II. This study showed significant improvement with therapeutic medial branch blocks in both groups in all aspects including functional status, drug intake, return to work, and improvement in the psychological status. This study showed that cumulative significant relief with 1 to 3 injections was $100 \%$ up to 1 to 3 months, $82 \%$ for 4 to 6 months, $21 \%$ for 7 to 12 months, and $10 \%$ after 12 months with a mean relief of $6.5 \pm 0.76$ months. There was significant difference noted in overall health status with improvement not only in pain relief, but also with physical, functional, and psychological status, as well as return to work status.

Manchikanti et al (182) evaluated the diagnostic validity and therapeutic value of lumbar facet joint nerve blocks with adjuvant agents. The study population consisted of 180 consecutive patients who were divided into three groups, with 60 patients in each group. The facet joints in all patients were investigated with diagnostic blocks using lidocaine $1 \%$, initially followed by bupivacaine $0.25 \%$ on separate occasions, usually 2 to 4 weeks apart, with or without the addition of Sarapin and/or methylprednisolone. All the patients who underwent double blocks with a definite response were considered as positive for facet joint mediated pain, yielding a prevalence of facet joint pain in chronic low back pain of $36 \%$ on average; however, the duration of pain relief associated with each injection by members of the three groups was significantly different. It was shown that patients who were finally judged to be positive for facet joint mediated pain showed mean cumulative relief with both the blocks of $20.6 \pm 3.97$ days, with a range of 3 to 98 days, in patients receiving local anesthetic; whereas it was $29.6 \pm 4.86$ days, with a range of 12 to 98 days, in patients receiving local anesthetic with Sarapin; compared to $49.8 \pm 9.04$ days, with a range of 5 to 160 days, in patients receiving local anesthetic, Sarapin, and methylprednisolone. Thus, this study showed that addition of adjuvant agents, either Sarapin with or without methylprednisolone, increased the duration of the relief and retained the diagnostic validity.

Marks et al (380) studied 86 patients with refractory chronic low back pain who were randomly assigned to receive either facet joint injections or facet nerve block, using local anesthetic and steroid. Using methylprednisolone acetate, $20 \mathrm{mg}$, along with lidocaine, $1.5 \mathrm{~mL}, 1 \%$, their results indicate good to excellent relief in $38 \%$ of patients following facet joint injection. A total of $25 \%$ of the patients achieved good to excellent relief after medial branch block immediately after infiltration. Good to excellent response was seen in $43 \%$ of patients receiving facet joint injection and $46 \%$ of patients with medial branch blocks in the first 2 weeks. At 1-month follow-up, 35\% of the patients with facet joint injection and $21 \%$ of the patients with medial branch blocks reported good relief. At 3-month followup, $22 \%$ of patients with facet joint injections showed good to excellent relief; and only $14 \%$ achieved the same level of relief following medial branch blocks.

In a prospective, randomized, single-blinded sequential analysis of 66 patients, Nash (379) reported comparable effectiveness of the medial branch of the posterior primary rami nerve blockade with reference to intra-articular injection of local anesthetic and steroid. He used $2 \%$ lidocaine, $1 \mathrm{~mL}$, and $0.5 \%$ bupivacaine, $1 \mathrm{~mL}$, for each medial branch, treating the nerve above and at the same

Table 7. Results of published reports of effectiveness of medial branch blocks

\begin{tabular}{|c|c|c|c|c|c|c|c|c|}
\hline \multirow[t]{2}{*}{ Study } & \multirow{2}{*}{$\begin{array}{c}\text { Study } \\
\text { Characteristics }\end{array}$} & \multirow{2}{*}{$\begin{array}{c}\text { No. of } \\
\text { Patients }\end{array}$} & \multirow{2}{*}{$\begin{array}{c}\text { No. of } \\
\text { Injections }\end{array}$} & \multirow{2}{*}{$\begin{array}{l}\text { Initial } \\
\text { Relief }\end{array}$} & \multicolumn{3}{|c|}{ Long-term Relief } & \multirow[t]{2}{*}{ Results } \\
\hline & & & & & 3 Months & 6 Months & 12 Months & \\
\hline Manchikanti et al (481) & $\mathrm{P}, \mathrm{RA}$ & 73 & $1-3$ & $100 \%$ & $100 \%$ & $82 \%$ & $21 \%$ & $P$ \\
\hline Manchikanti et al (481) & $\mathrm{P}, \mathrm{RA}$ & 73 & $1-10$ & $100 \%$ & $100 \%$ & $100 \%$ & $95 \%$ & $P$ \\
\hline Manchikanti et al (182) & P, RA, D & 180 & 2 & $100 \%$ & NA & NA & NA & $P$ \\
\hline Nash (379) & P, RA, D & 66 & 1 & $58 \%$ & NA & NA & NA & $N$ \\
\hline Marks et al (380) & $\mathrm{P}, \mathrm{RA}, \mathrm{D}$ & 86 & 1 & $46 \%$ & $14 \%$ & NA & NA & $N$ \\
\hline
\end{tabular}

$\mathrm{P}=$ prospective; $\mathrm{RA}=$ randomized; $\mathrm{D}=$ diagnostic blocks only; $\mathrm{NA}=$ not available; $P=$ positive; $N=$ negative 
level. For intra-articular injection he used 2\% lidocaine, 1 $\mathrm{mL}$, and $0.5 \%$ bupivacaine, $1 \mathrm{~mL}$, along with a $20-\mathrm{mg}$ methylprednisolone acetate suspension. The two treatments were equally effective but were disappointing in their therapeutic effect. A total of $58 \%$ of patients in each group demonstrated significant pain relief at 1-month follow-up. Based on this report, as a therapeutic measure, posterior ramus medial branch nerve blockade was proven to be as effective as intra-articular injection of steroid in low back pain of probable facet origin, suggesting that facet joint pain does not have an inflammatory component.

In another study, North et al (377) used diagnostic facet blocks and incorporated assessment by a disinterested third party. Following the diagnostic medial branch blocks, $42 \%$ of the patients reported at least $50 \%$ relief of pain. Among 40 patients who underwent temporary blocks but did not undergo radiofrequency denervation, $13 \%$ reported relief of at least $50 \%$ at long term follow-up with mean interval of 3.2 years.

Barnsley and Bogduk (375) studied 16 consecutive patients with chronic neck pain from motor vehicle accidents and reported complete or definite relief of their pain in 11 patients.

All of the trials described above face criticism. The randomized clinical trial by Manchikanti et al (481) is limited by failure to incorporate a placebo group and to utilize a major instrument to evaluate the progress. Other studies by Manchikanti et al (182), Marks et al (380), and Nash (379) were also limited by failure to incorporate a placebo group, lack of long term follow-up, and lack of reporting of outcomes.

Of the four controlled reports evaluating medial branch blocks, one study evaluating the therapeutic role was of moderate quality (481). The remaining three studies were of low quality for therapeutic purposes $(182,379,380)$.

In analyzing the type and strength of evidence due to the availability of only a total of four controlled studies for consideration, the evidence from two observational studies was also utilized. The analysis of type and strength of efficacy evidence shows that medial branch blocks provide level III (moderate) evidence. Level III - moderate evidence is defined as evidence obtained from well-designed trials without randomization, single group pre- post, cohort, time series, or matched case controlled studies.
Multiple investigators have studied the effectiveness of radiofrequency denervation of medial branches in the spine. Percutaneous radiofrequency neurotomy is a procedure that offers temporary relief of pain by denaturing the nerves that innervate the painful joint (482), but the pain returns when the axons regenerate. Fortunately, relief can be reinstated by repeating the procedure. Radiofrequency neurolysis as a treatment of chronic intractable pain began in the early 1930 s. Shealy $(483,484)$ pioneered spinal facet rhizotomy in the 1970s, and Sluijter and Koetsveld-Baart (319) initiated minimally invasive radiofrequency lesioning for pain of spinal origin.

Numerous reports describe the technique and effectiveness of radiofrequency thermoneurolysis $(319,377,482-511)$. Neurolytic blocks (512) and cryogenic neurolysis (513) also have been described. Success with radiofrequency neurotomy has been reported in the range of $17 \%$ to $90 \%$ for management of lumbar facet joint pain. There were four prospective randomized studies by Lord et al (487), Van Kleef et al (488) Dreyfuss et al (510), and Gallagher et al (510).

Lord et al (482) conducted a prospective, double blinded, placebo-controlled study of percutaneous radiofrequency neurotomy for management of chronic cervical facet joint pain. Lord et al (482) compared percutaneous radiofrequency neurotomy, in which multiple lesions were made and the temperature of the electrode was raised to $80^{\circ} \mathrm{C}$, with a control treatment using a procedure that was identical except for the facet that the radiofrequency current was not turned on. This study included 24 patients ( 9 men and 15 women) with a mean age of 43 years who presented with pain in one or more cervical facet joints after motor vehicle injury. The mean duration of pain was 34 months. Facet joint pain was diagnosed with the use of double-blinded, placebo-controlled local anesthetic blocks. The results showed that the median time that elapsed before the pain returned to at least $50 \%$ of the preoperative level was 263 days in the active treatment group and 8 days in the control group. At 27 weeks, seven patients in the active treatment group and one patient in the control group were free of pain. The authors concluded that, in patients with chronic cervical facet joint pain confirmed by double-blinded, placebo-controlled local anesthesia, percutaneous radiofrequency neurotomy with multiple lesions of target nerves could provide lasting relief.

Van Kleef et al (487), in a randomized trial of radiofre- 
quency lumbar facet denervation for chronic low back pain, studied 31 patients with a history of at least one year of chronic low back pain and facet pathology on the basis of a positive response to a diagnostic nerve blockade. Patients were subsequently randomly assigned to one of the two treatment groups. Each patient in the radiofrequency treatment group (15 patients) received an $80^{\circ}$ radiofrequency lesion of the dorsal ramus of the segmental nerve roots, L3, L4, and L5. In contrast, patients in the control group (16 patients) underwent the same procedure but without the use of radiofrequency current. Both the treating physician and the patients were blinded to the group assignment. A blinded investigator evaluated physical impairment, pain rating, degree of disability, and quality of life. The results showed that, 8 weeks after treatment, there were 10 successful treatments in the radiofrequency group and 6 in the control group. After 3, 6, and 12 months, the number of successes in the lesion and sham groups was 9 and 4, 7 and 3, and 7 and 2, respectively. This study results demonstrated that radiofrequency denervation of the lumbar facet joints can be effective for pain reduction in patients with lumbar facet joint pain.

Dreyfuss et al (488) examined the role of lumbar radiofrequency neurotomy for chronic zygapophysial facet joint pain in a pilot study using medial branch blocks. Their inclusion criterion was greater than $80 \%$ pain relief following two separate sets of medial branch blocks. The first set was performed with $0.5 \mathrm{~mL}$ of $2 \%$ lidocaine, and the second set with $0.5 \mathrm{~mL}$ of $0.5 \%$ bupivacaine. Treatment was successful, and statistically significant improvement was shown in the VAS scores, the Roland-Morris disability scale, physical function and bodily pain subscales of the SF-36 questionnaire, and the McGill pain questionnaire. Overall treatment success, defined as $50 \%$ or more pain relief at 1-year postneurotomy, was achieved in $87 \%$ of patients. The investigators noted that, even in patients who suffered with pain for more than five years, radiofrequency neurotomy of the medial branch nerves proved helpful, cost effective, and less time consuming than other interventions, such as exercised-based physical therapy or manipulative care.

Gallagher et al (510) studied 60 patients in a prospective manner by identifying those who had low back pain for more than 3 months for radiofrequency neurotomy. They used screening blocks as inclusion criteria for denervation with $0.5 \mathrm{cc}$ of $0.5 \%$ bupivacaine "into and around appropriate joints" under fluoroscopy. Of the 60 initial patients, 30 patients had a good response, and 11 had an equivocal response. The 30 patients with good response were ran- domly divided into four groups and received either medial branch radiofrequency neurotomy at $80^{\circ} \mathrm{C}$ for 90 seconds with active denervation, or a placebo. Statistically significant improvement was shown in the active denervation group compared with the placebo group. At 6-month follow up, however, only $24 \%$ of the patients with active denervation and $3 \%$ of the patients with placebo showed significant improvement.

All of the controlled studies faced criticism. All of them had a very small number of patients. In addition, Van Kleef et al (487) utilized a single block for a diagnosis of facet joint mediated pain. Further, Van Kleef et al (487) and Dreyfuss et al (488) included a number of patients with VAS scores at low levels. Many of the patients in both of the studies of Van Kleef et al (487) and Dreyfuss et al (488) were also young and working. Dreyfuss et al (488) recruited the patients by advertising and failed to incorporate a control or placebo group.

Among the observational reports, King and Lagger (511) looked at 60 patients with chronic low back pain undergoing radiofrequency neurotomy of the medial branches, which provided greater than $50 \%$ pain relief in only $27 \%$ of the patients. North et al (377) reviewed their experience with percutaneous radiofrequency denervation at a mean follow-up interval of 3.2 years, reporting at least $50 \%$ relief of pain at long term follow up. In another study, Sluijter (491) studied the use of radiofrequency lesioning for pain relief in failed low back surgery syndrome. They defined the success as better than $50 \%$ relief and reported that percutaneous facet denervation had a success rate of $40 \%$ in these patients as opposed to $80 \%$ in those who did not undergo back surgery. Ogsbury et al (509) reported results of radiofrequency rhizotomies in 71 patients; $35 \%$ of the patients showed a successful long term result. Sluijter and Koetsveld-Baart (319) studied the effectiveness of percutaneous facet denervation in 64 patients with cervical pain syndromes and reported good results in $41 \%$ of the patients. Schaerer $(502,505)$ reported good pain relief in $50 \%$ of the patients. Rashbaum (489) studied 100 patients with radiofrequency neurotomy, reporting relief in $82 \%$ of the patients at 3 to 6 months, and $68 \%$ at 3 years.

The studies by Lord et al (482) and Van Kleef et al (487) were double-blinded and placebo controlled. They were also considered as high quality. The remaining two studies by Dreyfuss et al (488) and Gallagher et al (510) were considered as low quality.

As shown in Table 8 , three of the four controlled trials, 
Table 8. Results of published reports on effectiveness of facet joint (medial branch) radiofrequency neurolysis

\begin{tabular}{|c|c|c|c|c|c|c|c|}
\hline \multirow[t]{2}{*}{ Study } & \multirow{2}{*}{$\begin{array}{c}\text { Study } \\
\text { Characteristics }\end{array}$} & \multirow{2}{*}{$\begin{array}{l}\text { No. of } \\
\text { Patients }\end{array}$} & \multirow{2}{*}{$\begin{array}{c}\text { Initial Relief } \\
\text { 1-4 Weeks }\end{array}$} & \multicolumn{3}{|c|}{ Long-term Relief } & \multirow[t]{2}{*}{ Results } \\
\hline & & & & 3 Months & 6 Months & 12 Months & \\
\hline Lord et al (482) & $\mathrm{P}, \mathrm{PC}, \mathrm{RA}, \mathrm{DB}$ & 24 & $75 \%$ & $58 \%$ & $58 \%$ & $50 \%$ & $P$ \\
\hline Van Kleef (503) & P, PC, RA, DB & 31 & $67 \%$ & $60 \%$ & $47 \%$ & $47 \%$ & $P$ \\
\hline Dreyfuss et al (504) & $\mathrm{P}, \mathrm{C}$ & 15 & $93 \%$ & $100 \%$ & $87 \%$ & $87 \%$ & $P$ \\
\hline Gallagher et al (510) & $\mathrm{P}, \mathrm{PC}, \mathrm{RA}$ & 60 & $42 \%$ & NA & $24 \%$ & NA & $N$ \\
\hline
\end{tabular}

$\mathrm{C}=$ controlled; $\mathrm{P}=$ prospective $; \mathrm{RA}=$ randomized $\mathrm{PC}=$ placebo controlled $\mathrm{DB}=$ double blind $\mathrm{NA}=$ not available $P=$ positive; $N=$ negative

and both randomized, placebo-controlled, double-blind studies showed the significant pain relief, along with improvement in other parameters, indicating strong evidence from multiple controlled trials. In addition, evidence from uncontrolled studies also supports the contention that radiofrequency is effective, even though (contrary to the popular belief), controlled trials showed better improvement than uncontrolled studies. Thus, the type and strength of efficacy evidence for radiofrequency neurotomy in managing facet joint mediated pain is level II - strong, defined as evidence from at least one properly designed randomized controlled trial of appropriate size and high quality or multiple adequate studies. In addition, in a randomized, double-blind placebo-controlled trial, Wallis et al (514) also showed resolution of psychological distress of whiplash patients following treatment by radiofrequency neurotomy.

\section{Epidural Injections}

Approaches available to access the epidural space are interlaminar (cervical, thoracic, and lumbar), transforaminal (cervical, thoracic, lumbar, and sacral), and caudal. Epidural steroid injections are the most commonly used interventional techniques in pain management clinics. In fact, the first reports of neural blockade in managing low back and lower extremity pain secondary to lumbar nerve root compression were of epidural injections caudally (307309). The first administration of epidural steroids was by transforaminal epidural injections, reported by Robechi and Capra in 1952 (315), and Lievre et al in 1957 (316). Access to the lumbar epidural space through a paramedian approach was proposed by Pages in 1921 (311). Lievre et al (316) reported their experience with injection of a hydrocortisone and contrast into the epidural space of 46 patients with sciatica in 1953 . They thought that 23 had good or very good results and 8 had mediocre results; and the rest were considered failures. The effects of caudal and interlaminar epidural steroid injections were first reported independently by Goebert and colleagues (317) and Brown (515) in 1960. Goebert and colleagues (317) administered three injections of procaine and hydrocortisone into the epidural space to 239 patients with sciatica, and reported greater than $60 \%$ relief of symptoms in $58 \%$ of the patients. Since that time, the technique and indications of epidural steroid injections have been changing constantly. Numerous reviews have appeared in the literature evaluating the effectiveness of epidural steroid injections.

The first systematic review of effectiveness of epidural steroid injections was by Kepes and Duncalf in 1985 (51). They concluded that the rationale for epidural systemic steroids was not proven. However, in 1986 Benzon (52), utilizing the same studies, concluded that mechanical causes of low back pain, especially those accompanied by signs of nerve root irritation, may respond to epidural steroid injections. The difference in the conclusion of Kepes and Duncalf (51) and Benzon (52) may be due to the fact that Kepes and Duncalf (51) included studies on systemic steroids whereas Benzon (52) limited his analysis to studies on epidural steroid injections only. The debate concerning the epidural steroid injections is also illustrated by the recommendations of the Australian National Health and Medical Research Council Advisory Committee on epidural steroid injections (47). In this report, Bogduk et al (47) extensively studied caudal, interlaminar, and transforaminal epidural injections, including all the literature available at the time, and concluded that the balance of the published evidence supports the therapeutic use of caudal epidurals but does not vindicate it. They also concluded that the results of lumbar interlaminar epidural steroids strongly refute the utility of epidural steroids in acute sciatica. 
Bogduk (57) updated recommendations in 1999, recommending against epidural steroids by the lumbar route as requiring too high a number necessary for treatment, but supporting the potential usefulness of transforaminal steroids for disc prolapse. In 1995, Koes et al (45) reviewed 12 trials of lumbar and caudal epidural steroid injections and reported positive results from only six studies. However, review of their analysis showed that there were five studies for caudal epidural steroid injections and seven studies for lumbar epidural steroid injections. Four of the five studies involving caudal epidural steroid injections were positive, whereas five of seven studies were negative for lumbar epidural steroid injections. Koes et al (46) updated their review of epidural steroid injections for low back pain and sciatica, including three more studies with a total of 15 trials which met the inclusion criteria. In this study, they concluded that of the 15 trials, eight reported positive results of epidural steroid injections. Benzon (516) and Benzon and Molly (60) considered the role of epidural steroid injections controversial but recommended the continued use of epidural steroid injections as part of the overall management of patients with acute radicular pain, herniated disc, or new radiculopathy superimposed on chronic back pain. Watts and Silagy (48) in 1995 performed a meta-analysis of the available data and defined efficacy in terms of pain relief (at least $75 \%$ improvement) in the short term (60 days) and in the long term (1 year). They concluded that epidural steroid injections increased the odds ratio of pain relief to 2.61 in the short term and to 1.87 in the long term (odds ratio greater than one suggests efficacy; equal to or greater than two suggests significant efficacy). Tulder et al (421), in analyzing numerous treatments based on scientific evidence in conservative treatment of chronic low back pain, also included seven studies of epidural steroid injections. They concluded that there was conflicting evidence with inconsistent findings with regards to the effectiveness of epidural steroid injections. McQuay and Moore (517) in 1998 reviewed the literature and concluded that epidural corticosteroid injections are effective for back pain and sciatica. They also concluded that, even though epidural steroid injections can optimize conservative therapy and provide substantial pain relief for up to 12 weeks in patients with acute or subacute sciatica, few patients with chronic pain report complete relief; the majority must return for repeated epidural injections. The perceived advantages of each of the three approaches include $(33,41,42,47,58,518-543)$ :

1. The interlaminar entry is directed more closely to the assumed site of pathology, facilitating delivery of the injectate directly to its target and re- quiring less volume;

2. The caudal entry is relatively easily achieved, with minimal risk of inadvertent dural puncture; and

3. The transforaminal approach is target specific in fulfilling the aim of reaching the primary site of pathology.

The disadvantages of each of the three approaches are illustrated in Table 9.

Due to the inherent variations, differences, advantages, and disadvantages applicable to each technique (including the effectiveness and outcomes), caudal epidural injections; interlaminar epidural steroid injections, (cervical, thoracic, and lumbar epidural injections), and transforaminal epidural injections (cervical, thoracic, and lumbosacral) are considered as an entity within epidural injections and are

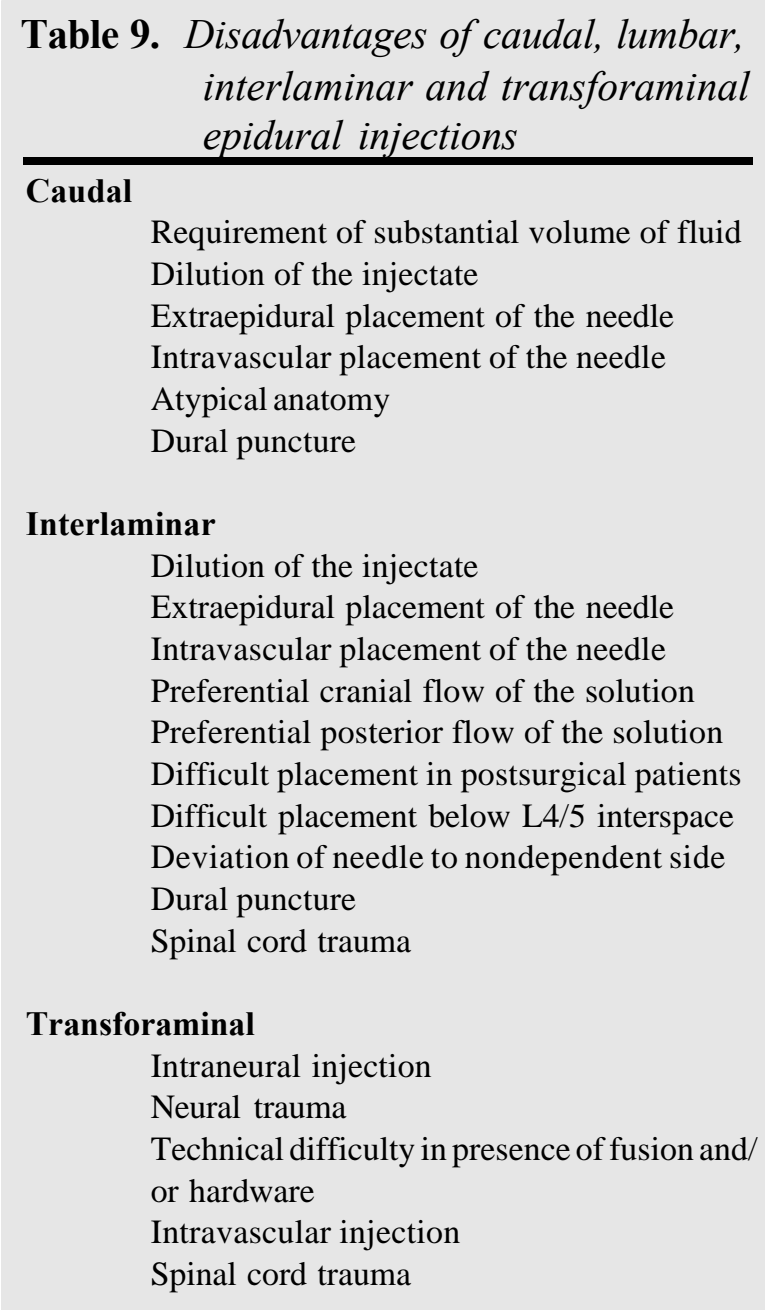

Modified and adapted from Manchikanti (58) 
discussed as such below.

Caudal Epidural Injections: Extensive literature available on caudal epidural injections includes six controlled studies (544-549) and numerous uncontrolled reports (543, 550-559).

Breivik et al (544) in a prospective, randomized, crossover study, evaluated 35 patients with chronic low back pain, allocated to treatment with up to three caudal epidural injections of bupivacaine and methylprednisolone or bupivacaine and normal saline at weekly intervals. The study followed a parallel, cohort design and allowed patients who failed to obtain relief with one of the treatments to receive the reciprocal treatment. During initial therapy $56 \%$ of patients receiving methylprednisolone experienced significant relief, compared to $26 \%$ with bupivacaine with saline. In the crossover, only one of seven patients who had methylprednisolone therapy got relief from the subsequent bupivacaine and saline injection (14\%), in contrast to $73 \%$ of patients who failed to respond to bupivacaine and saline injection reported satisfactory relief after receiving the methylprednisolone injection. While $50 \%$ of the patients treated with steroids returned to work, $20 \%$ of the patients treated with bupivacaine returned to work.

Bush and Hillier (545) in a double-blind, randomized evaluation studied 23 patients with lumbar radicular pain allocated either to receive two caudal epidural injections of either a $25 \mathrm{~mL}$ mixture of normal saline, procaine and 80 $\mathrm{mg}$ triamcinolone, or $25 \mathrm{~mL}$ of normal saline alone. Patients were assessed for pain levels, improvement in straight-leg raise, and lifestyle. The follow-up, at four weeks demonstrated significantly greater pain relief and mobility with a significantly improved quality of life following triamcinolone injection. However, at one year follow-up while the treated patients showed greater improvement than placebo patients, the significant difference was limited to straight-leg raise tolerance.

In contrast to the above studies, Beliveau (547) found no difference in pain relief between 24 patients treated with caudal injections of $40 \mathrm{~mL}$ of $1 \%$ procaine and $80 \mathrm{mg}(2$ $\mathrm{mL}$ ) of methylprednisolone, and an equal number of patients treated with $42 \mathrm{~mL}$ of procaine alone. The patients in this study had moderate or severe unilateral sciatica, thought to be caused by an intervertebral disc lesion with or without neurological signs. They assessed the effect of the injection a week later according to the symptoms and the findings of physical examination. Injections were re- peated if improvement was seen after the first injection, with a total of 82 injections for 48 patients. One to three months later they saw complete relief in $42 \%$ of the patients in the steroid group, and in $29 \%$ in the normal saline group. This study demonstrated the efficacy of caudal epidural injections in sciatica with or without steroids. It failed, however, to demonstrate superiority of steroids over local anesthetic except in cases of long standing severe sciatica.

Yates (549) treated patients with low back pain and sciatica by epidural injection of normal saline or $0.5 \%$ lignocaine, with or without triamcinolone given at weekly intervals in random order. Subjective and objective criteria of progress were measured. Greatest improvement was noted after the injection containing steroid. Lignocaine $0.5 \%$, and normal saline used individually produced less marked improvement. No specific benefits of local anesthesia were found other than comfort during injection. His report did not address pain relief but focused on improvement in straight leg raising, which seemed to correlate with pain relief.

Matthews et al (546) compared the responses of patients treated with caudal epidural injections of bupivacaine and methylprednisolone or a control injection of $2 \mathrm{~mL}$ of lignocaine over the sacral hiatus. At assessment after one month, there was no significant difference between the two groups. However, at three months, the treated group was reported to be significantly more pain free.

Czarski (547) evaluated the use of caudal epidural injections comparing novocaine and hydrocortisone and procaine hydrochloride alone in the treatment of patients with prolapsed lumbar intervertebral disc, with 60 patients in procaine hydrochloride group and 123 patients in procaine hydrochloride and hydrocortisone group. $\mathrm{He}$ demonstrated statistically significant and clinically significant differences in outcomes comparing the use of caudal epidural injections. Unfortunately, however, the duration of follow-up was not specified even though complete relief was reported in 22 of the 123 patients, with significant relief in 64 of 123 patients; whereas marginal relief was reported in 14 patients with no relief or patients getting worse on 23 occasions in hydrocortisone group. In comparison, in procaine hydrochloride group, 8 of 60 patients obtained significant relief, none of the patients obtaining complete relief, 35 obtaining marginal relief and 17 patients getting no relief or becoming worse.

Numerous uncontrolled reports on the use of caudal epi- 
Table 10. Results of published reports on caudal epidural steroid injections

\begin{tabular}{|c|c|c|c|c|c|c|c|c|}
\hline \multirow[t]{2}{*}{ Study } & \multirow[t]{2}{*}{$\begin{array}{c}\text { Study } \\
\text { Characteristics }\end{array}$} & \multirow[t]{2}{*}{$\begin{array}{l}\text { No. of } \\
\text { Patients }\end{array}$} & \multirow[t]{2}{*}{$\begin{array}{c}\text { Drugs } \\
\text { Utilized }\end{array}$} & \multirow[t]{2}{*}{$\begin{array}{l}\text { No. of } \\
\text { Injections }\end{array}$} & $\begin{array}{c}\text { Initial Relief } \\
\text { Control vs. } \\
\text { Treatment }\end{array}$ & \multicolumn{2}{|c|}{$\begin{array}{c}\text { Long-term Relief } \\
\text { Control vs. } \\
\text { Treatment }\end{array}$} & \multirow[t]{2}{*}{ Results } \\
\hline & & & & & 3-4 Weeks (\%) & 3 Months (\%) & 6 Months (\%) & \\
\hline Breivik et al (544) & $\mathrm{P}, \mathrm{RA}, \mathrm{DB}$ & 35 & S, LA, NS & $1-3$ & 25 vs. 63 & 20 vs. 50 & 20 vs. 50 & $P$ \\
\hline Bush and Hillier (545) & P, RA, PC, DB & 23 & NS, LA, S & 2 & 100 & N/A & 64 vs. 83 & $P$ \\
\hline Yates (549) & $\mathrm{P}, \mathrm{RA}, \mathrm{PC}, \mathrm{DB}$ & 20 & S, NS, LA & $1-4$ & N/A & N/A & N/A & $P$ \\
\hline Matthews et al (546) & $\mathrm{P}, \mathrm{RA}, \mathrm{PC}$ & 34 & S, LA & $1-3$ & 56 vs 67 & SMPR & N/A & $P$ \\
\hline Czarski (548) & P, RA & 183 & S, LA & N/A & 13 vs 72 & N/A & N/A & $P$ \\
\hline Beliveau (547) & $\mathrm{P}, \mathrm{RA}$ & 48 & LA, S & $1-2$ & 70 vs. 75 & 70 vs. 75 & N/A & $N$ \\
\hline
\end{tabular}

$\mathrm{P}=$ prospective; $\mathrm{RA}=$ randomized $; \mathrm{PC}=$ placebo controlled; $\mathrm{DB}=$ double blind $; \mathrm{LA}=$ local anesthetic; $\mathrm{NS}=$ normal saline; $\mathrm{S}=$ steroids; $\mathrm{SMPR}=$ significantly more pain relief; N/A = not available; $P=$ positive; $N=$ negative

dural injections have shown favorable response with respectable benefit $(313,317,550-559)$. In 1930, Evans (313) reported a cure rate of $61 \%$ after injecting large volumes of procaine and saline to treat sciatica. The first uncontrolled study of epidural steroids with 86 patients receiving caudal epidural injections reported greater than $60 \%$ relief of pain in $72 \%$ of patients (317). Mount et al (556) reported greater than $85 \%$ relief in $65 \%$ of the patients suffering with lumbar intervertebral disc syndrome. Cyriax (551) reported his extensive experience with 20,000 patients, who showed significant improvement. Ciocon et al (552) studied the efficacy of caudal epidural blocks for elderly patients with lumbar canal stenosis. In this descriptive, prospective study, 30 patients with a mean age of 76 \pm 6.7 years with leg pain were studied, with a 10-month follow-up evaluation utilizing Roland's five point pain rating scale. They were treated with a total of three injections of $0.5 \%$ Lidocaine with $80 \mathrm{mg}$ of methylprednisolone administered at weekly intervals. The results showed significant pain reduction up to 10 months from a mean pain level of $3.4 \pm 0.82$ to a mean level of $1.5 \pm 0.86$, with satisfactory relief in $90 \%$ of patients. Manchikanti et al (553), in evaluating the effectiveness of caudal epidural steroid injections under fluoroscopic visualization, showed significant improvement that was better than that of blind lumbar interlaminar epidural injections. Sharma (557) studied 201 patients with lumbago, sciatica, backache with sciatica, and other conditions reporting favorable results in $56 \%$ of the patients.

The quality of randomized, controlled studies for caudal epidural injections is considered as high quality for four of the six studies (544-547) and of low for the two $(548,549)$. As shown in Table 10, the data from six of the controlled studies show positive effect in five studies. In addition, multiple systematic reviews were also favorable for caudal epidural steroid injections (45-47). Multiple observational studies also provided favorable results consistent with controlled trials. The type and strength of efficacy evidence is of level II - strong, with research-based evidence from at least one properly designed randomized controlled trial of appropriate size and high quality or multiple adequate scientific studies.

Interlaminar Epidural Injections: Interlaminar epidural injections may be administered either in the cervical, thoracic, or lumbar regions. Studies in the literature evaluating the efficiency of interlaminar epidural injections, specifically the lumbar epidural injections, are extensive. This includes ten controlled studies involving lumbar epidural steroid injections $(258,560-568)$; but only three controlled studies involving cervical interlaminar epidural injections (569-571); along with multiple uncontrolled studies and case reports (571-612).

Dilke et al (561) treated 100 patients with unilateral sciatica with either lumbar epidural injection with $80 \mathrm{mg}$ of methylprednisolone and $10 \mathrm{~mL}$ of normal saline or an injection of $1 \mathrm{~mL}$ of normal saline into an interspinous ligament. All patients received physical therapy with hydrotherapy and exercise. Follow-up was at two weeks and three months, measuring time of bed rest, days of hospitalization, pain relief, consumption of analgesics, and resumption of work three months later. Sixty percent of the patients in the treated group and $31 \%$ in the control group improved immediately after the injections. A greater proportion of actively treated patients had no pain at three months, took no analgesics, resumed work, and fewer of 
them underwent subsequent surgery or other non-surgical treatment. Ninety-one percent of the patients in the treated group improved at three months, whereas $74 \%$ of the patients in the control group improved; however; there was only one patient in the treated group with severe pain, in contrast to six in the control group (2\% vs. $16 \%$ ).

Ridley et al (564) corroborated the findings of Dilke et al (561) in 35 patients with sciatica in a randomized study that compared an epidural injection of $80 \mathrm{mg}$ of methylprednisolone in $10 \mathrm{~mL}$ of normal saline to injection of 2 $\mathrm{mL}$ of normal saline into interspinous ligament. They reported improvement in $90 \%$ of the patients in the treated group compared to $19 \%$ in the control group at one and two weeks following treatment, which was maintained up to 12 weeks but deteriorated by 24 weeks to pre-treatment levels.

Carette et al (258) in a randomized, double-blind trial administering up to three epidural injections of methylprednisolone acetate ( $80 \mathrm{mg}$ and $8 \mathrm{~mL}$ of isotonic saline) or isotonic saline $(1 \mathrm{~mL})$ to 158 patients with sciatica due to a herniated nucleus pulposus, reported negative results. The patients were evaluated utilizing Oswestry Disability Scores with follow-up at 3,6, and 12 weeks after treatment. There were 78 patients in the treatment group and 80 patients in the placebo group, with L4/5 disc herniation in $50 \%$ and L5/S1 disc herniation in $46 \%$ of the patients. After 6 weeks, a significant difference was seen with improvement in leg pain in the methylprednisolone group. However, after 3 months, there were no significant differences between groups. At 12 months, the cumulative probability of back surgery was equal in both groups.

Snoek et al (567) studied 51 patients with lumbar root compression documented by neurological deficit and a concordant abnormality noted on myelography. They compared the effects of $80 \mathrm{mg}$ of methylprednisolone $(2 \mathrm{~mL})$ and $2 \mathrm{~mL}$ of normal saline injected into the epidural space by the lumbar route. They found no significant differences between the two groups with respective relief of pain and a variety of physical parameters.

Cuckler et al (560), in a prospective, randomized, doubleblind trial, evaluated 73 patients, comparing $7 \mathrm{~mL}$ of methylprednisolone ( $80 \mathrm{mg}$ with procaine) and $7 \mathrm{~mL}$ of normal saline with procaine. The patients were suffering with radicular pain due to either acute herniated nucleus pulposus or spinal stenosis. They reported no significant differences in outcomes. This study was considered negative, condemning lumbar epidural steroid injections.
Klenerman et al (563) randomized patients with sciatica into four treatment groups: epidural steroid injection, epidural saline, epidural bupivacaine and needling with a Touhy needle inserted into the interspinous ligament. The results were the same in the four treatment groups, with approximately $75 \%$ of the patients responding to the treatments.

Serrao et al (566) evaluated the effectiveness of epidural steroid injections compared to subarachnoid midazolam in mechanical low back pain, concluding that epidural steroid injections are comparable to subarachnoid midazolam in patients with mechanical low back pain.

Stav et al (570) studied 52 patients with chronic, resistant cervical brachialgia in a randomized, controlled study. They divided patients into two groups, with 25 patients in Group A who were treated with cervical epidural steroid and lidocaine injections, and 17 patients in Group B who were treated with steroid and lidocaine injections into the posterior neck muscles. One to three injections were administered at two week intervals, according to the clinical response. All patients continued with their various prestudy treatments: nonsteroidal anti-inflammatory drugs, nonopioid analgesics, and physiotherapy. One week after the last injection, very good and good pain relief were reported in $76 \%$ of the patients in Group A, as compared to $36 \%$ of the patients in Group B. At one year $68 \%$ of the Group A patients continued to have very good and good pain relief, whereas only $12 \%$ of Group B patients reported similar pain relief, with statistically significant differences. They also reported that they were unable to achieve significant improvement of tendon reflexes or of sensory loss in both groups; but the increase in the range of motion, the percentage of the patients who were able to decrease their daily dose of analgesics, and recovery of the capacity for work was significantly better in Group A.

Castagnera et al (569) evaluated long term results of cervical epidural steroid injection, with and without morphine, in chronic cervical radicular pain in 24 patients, without need of surgery, but suffering for more than 12 months from cervical radicular pain, in a prospective randomized study. The patients were randomly allocated into two groups: the steroid group, with 14 patients receiving an equivalent volume of $0.5 \%$ lidocaine plus triamcinolone acetonide $(10 \mathrm{mg}$ per $\mathrm{mL})$ and the steroid plus morphine group, with 10 patients receiving the same combination plus $2.5 \mathrm{mg}$ of morphine sulfate. The success rate was $79 \%$ in the steroid group and $80 \%$ in the steroid plus morphine group. They reported an initial success rate of $96 \%$, 
followed by a $75 \%$ success rate in one month, $79 \%$ success rate at three months, $79 \%$ at six months, and $79 \%$ at 12 months.

Bush and Hillier (571) described the response to cervical epidural steroid injections of 68 patients with neurologic deficits of two months duration and an abnormal MRI in a prospective study with independent clinical review. They initially utilized a nonfluoroscopically guided lateral approach at C7. If significant improvement was not seen after the first injection, a repeat injection was performed transforaminally, with fluoroscopic guidance within one month. Similarly, a third injection was performed if needed in the same manner as the second injection. An average of 2.5 injections per patient was required for adequate pain control. Overall, $93 \%$ of the patients reported pain relief lasting seven months.

Among the remaining controlled trials, Helliwell (562) studied 20 patients utilizing normal saline and steroid with one to three injections reporting $70 \%$ positive results at three months; Rogers et al (568) studied 30 patients utilizing steroid and local anesthetic with one injection reporting only $48 \%$ positive results compared to $20 \%$ in the control group; and Rocco et al (565) studied 22 patients uti- lizing local anesthetic and steroids with one to three injections reporting only $13 \%$ relief at three months.

Fukusaki et al (580) concluded that epidural steroid injections had no beneficial effects on the pseudoclaudication associated with spinal canal stenosis as compared with local anesthetic alone. Fifty-three patients with pseudoclaudication were randomly divided into three groups: Group I $(n=16)$ underwent epidural injection with $8 \mathrm{~mL}$ of saline; Group $2(\mathrm{n}=18)$ underwent epidural injection of $8 \mathrm{~mL}$ of $1 \%$ mepivacaine; Group $3(\mathrm{n}=19)$ underwent epidural injection with a combination of $8 \mathrm{~mL}$ of $1 \%$ mepivacaine and $40 \mathrm{mg}$ of methylprednisolone, with each patient receiving a total of three injections during the first week. After one week, $12.5 \%$ of patients in Group 1, 55.5\% of patients in Group 2, and $63.2 \%$ of patients in Group 3 showed good or excellent result. However, at one month and three months the improvement deteriorated to $6.5 \%$ in Group 1, 16.7\% and 5.6\% in Group 2, and $15.8 \%$ and $5.3 \%$ in Group 3.

Most of the control studies faced criticism. Dilke et al (561) and Ridley et al (564) were criticized for the lack of epidural anesthetic and limited outcome measures, as well as for early crossover and a small sample size in Ridley's

Table 11. Results of published reports on interlaminar lumbar and cervical epidural steroid injections

\begin{tabular}{|c|c|c|c|c|c|c|c|c|}
\hline \multirow[t]{2}{*}{ Study } & \multirow[t]{2}{*}{$\begin{array}{l}\text { No. of } \\
\text { Patients }\end{array}$} & \multirow[t]{2}{*}{$\begin{array}{c}\text { Study } \\
\text { Characteristics }\end{array}$} & \multirow[t]{2}{*}{$\begin{array}{c}\text { Drugs } \\
\text { Utilized }\end{array}$} & \multirow[t]{2}{*}{$\begin{array}{c}\text { No. of } \\
\text { Injections }\end{array}$} & \multirow{2}{*}{$\begin{array}{c}\begin{array}{c}\text { Initial Relief } \\
\text { Control vs. } \\
\text { Treatment }\end{array} \\
\text { 3-4 Weeks (\%) }\end{array}$} & \multicolumn{2}{|c|}{$\begin{array}{l}\text { Long-term Relief } \\
\text { Control vs. } \\
\text { Treatment }\end{array}$} & \multirow[t]{2}{*}{ Results } \\
\hline & & & & & & 3 Months (\%) & 6 Months (\%) & \\
\hline Dilke et al (560) & 100 & $\mathrm{P}, \mathrm{PC}$ & $\mathrm{NS}, \mathrm{S}$ & $1-2$ & 31 vs. 60 & 74 vs. 91 & N/A & $P$ \\
\hline Ridley et al (564) & 35 & $\mathrm{P}, \mathrm{RA}, \mathrm{PC}$ & NS, S & $1-2$ & 19 vs. 90 & 19 vs. 90 & 65 & $P$ \\
\hline Helliwel et al (562) & 20 & $\mathrm{P}, \mathrm{C}, \mathrm{PC}$ & NS, S & $1-3$ & 70 & 70 & N/A & $P$ \\
\hline Stav et al (570) & 42 & $\mathrm{P}, \mathrm{RA}, \mathrm{PC}$ & LA, S & $1-3$ & 76 & 68 & 68 & $P$ \\
\hline Castagnera (569) & 24 & P, RA & LA, S, M & 1 & 75 & 79 & 79 & $P$ \\
\hline Serrao et al (566) & 28 & $\mathrm{P}, \mathrm{C}$ & NS, S & 2 & 71 & 71 & N/A & $N$ \\
\hline Klenerman et al (563) & 63 & $\mathrm{P}, \mathrm{RA}, \mathrm{PC}$ & NS, S, LA & 1 & 79 & N/A & N/A & $N$ \\
\hline Rogers et al (568) & 30 & $\mathrm{P}, \mathrm{C}$ & S, LA & 1 & 20 vs. 48 & N/A & N/A & $N$ \\
\hline Rocco et al (565) & 22 & $\mathrm{P}, \mathrm{C}$ & LA, S & $1-3$ & N/A & 13 & N/A & $N$ \\
\hline Cuckler et al (560) & 73 & $\mathrm{P}, \mathrm{RA}, \mathrm{DB}, \mathrm{PC}$ & S, LA, S & $1-2$ & 26 vs 40 & N/A & 13 vs. 26 & $N$ \\
\hline Snoek et al (567) & 51 & $\mathrm{P}, \mathrm{PC}$ & NS, S & 1 & 25 vs. 33 & N/A & N/A & $N$ \\
\hline Carette et al (258) & 158 & $\mathrm{P}, \mathrm{RA}, \mathrm{DB}, \mathrm{PC}$ & NS, S & $1-3$ & 29 vs. 33 & No sig. diff. & No sig. diff. & $N$ \\
\hline Bush and Hillier (571) & 68 & $\mathrm{P}, \mathrm{C}$ & LA, S & $1-6$ & 76 & 76. & 76 & $P$ \\
\hline
\end{tabular}

$\mathrm{P}=$ prospective $\mathrm{C}=$ controlled; $\mathrm{PC}=$ placebo controlled; $\mathrm{RA}=$ randomized; $\mathrm{DB}=$ double-blind; $\mathrm{LA}=$ local anesthetic;

$\mathrm{S}=$ steroids; $\mathrm{NS}=$ normal saline; $\mathrm{M}=$ morphine; $\mathrm{N} / \mathrm{A}=$ not available $P=$ positive $N=$ negative; vs = versus 
study (564). Carette et al (258) also failed to include local anesthetic in the injection. In addition, Carette et al (258) used the same target level of epidural injection in all patients irrespective of location or pathology and discounted the short term facilitating effects of epidural steroid injections. There were also no structured co-interventions (258). Cuckler et al (560) included patients with prior surgery. They also evaluated responses at 24 hours which was felt to be inappropriate as it was quite a short period over which to evaluate the effectiveness of an invasive procedure and the anti-inflammatory effect of long-acting steroid preparation. In addition, they also made injections at the L3/4 level in all of the patients rather than injecting close to the site of pathology. Bush and Hillier (571) study was not randomized and there were no outcome parameters. Fukusaki et al (580) utilized three epidural injections in a one week period with no pharmacologic basis, and failed to enter the epidural space in a significant number of patients. Interestingly none of the controlled studies were performed under fluoroscopic visualization.

Numerous uncontrolled trials reported good results in $18 \%$ to $90 \%$ of patients receiving lumbar epidural steroid injections. Berman et al (583) reported good to excellent results at three months, six months, and one year in $87 \%$, $77 \%$ and $69 \%$ of patients, respectively. Brown (572) reported even better results with $80 \%$ relief at one year. Other selective uncontrolled trials also reported six months of relief in approximately $60 \%$ of the patients, and 1 year relief in 36\% of the patients (573-578). Pawl et al (588), in evaluating the records of 136 patients with typical radicular symptoms reported that 29 patients or $80 \%$ indicated that the relief of pain from epidural steroid injection was $50 \%$ or more, and $50 \%$ of the patients were able to avoid surgery with the help of epidural injections. Various other evaluations have shown success rates with cervical epidural injections varying from $64 \%$ to $79 \%$ for less than three months, $50 \%$ to $68 \%$ for 3 to 6 months, and $25 \%-68 \%$ for over 6 months $(584-586,591)$. Manchikanti et al (553) compared blind lumbar interlaminar epidural steroid injections with fluoroscopically directed caudal and transforaminal injections and concluded that blind interlaminar epidural injections were not cost effective.

In terms of quality of the 13 studies considered in the interlaminar lumbar and cervical epidural steroid injections, two were of high quality $(258,567)$; six were of moderate quality $(560,561-563,566,568)$, whereas remaining five were of low quality $(564,565,569-571)$. Of the 13 studies, three of nine interlaminar lumbar epidural steroid injections and three of three cervical epidural steroid injec- tions were judged to be positive, while the remaining were considered negative (Table 11). Thus, evidence from controlled studies is predominantly negative for lumbar interlaminar and positive for cervical interlaminar epidural injections. However, multiple observational studies showed positive results. Hence, type and strength of efficacy evidence is level III to IV moderate to limited. Level III - moderate is defined as evidence from well-designed trials without randomization, single group pre-post, cohort, time series, or matched case controlled studies. Level IV - limited is defined as evidence from well-designed nonexperimental studies from more than one center or research group.

Transforaminal Epidural Injections: Caudal epidural injection of drugs was introduced as the first type of entry into the epidural space in 1901, and transforaminal epidural injection was introduced as the first and earliest use of epidural steroids $(315,316)$. In 1952, Robechhi and Capra (315) administered periradicular injection of hydrocortisone into the first sacral nerve root and reported relief of lumbar and sciatic pain in a woman in the Italian literature. Subsequently, Lievre and colleagues (316) also reported transforaminal injection of steroids into the first sacral nerve root, in the French literature. The sacral transforaminal epidural injection of steroids was popularized largely in the Italian literature $(315,613-618)$, and to a lesser extent, in the French literature (316, 619-621). There were no significant American reports until 1971, when McNab described the diagnostic value of selective nerve root infiltration in patients with suspected radicular etiology of pain (405). In contrast to reports of caudal and interlaminar epidural injections, reports of transforaminal injections are sparse in the literature (622-631). Review of the literature showed three prospective, randomized controlled, trials (622-624); two prospective evaluations $(571,625)$; and multiple retrospective studies $(415,553$, 609, 626-628) (Table 12).

Riew et al (622), in a prospective, randomized, controlled, double-blinded study, evaluated the effectiveness of transforaminal epidural cortical steroids in subjects with disc herniations and/or spinal stenosis. The study included 55 patients with disc herniations or spinal stenosis referred for surgical evaluation. All subjects had clinical indications for surgery, and radiographic confirmation of nerve root compression. All had failed a minimum of 6 weeks of conservative care or had unrelenting pain. Exclusion criteria consisted of patients who had sustained trauma, patients with evidence of other serious diseases, patients demonstrating adverse reactions to the medications employed 
Table 12. Results of published reports on lumbar and cervical transforaminal epidural steroid injections

\begin{tabular}{|c|c|c|c|c|c|c|c|c|}
\hline \multirow[t]{2}{*}{ Study } & \multirow[t]{2}{*}{$\begin{array}{c}\text { Study } \\
\text { Characteristics }\end{array}$} & \multirow[t]{2}{*}{$\begin{array}{l}\text { No. of } \\
\text { Patients }\end{array}$} & \multirow[t]{2}{*}{$\begin{array}{c}\text { Drugs } \\
\text { Utilized }\end{array}$} & \multirow[t]{2}{*}{$\begin{array}{c}\text { No. of } \\
\text { Injections }\end{array}$} & \multirow{2}{*}{$\begin{array}{c}\begin{array}{c}\text { Initial Relief } \\
\text { Control vs. } \\
\text { Treatment }\end{array} \\
\text { 3-4 Weeks (\%) }\end{array}$} & \multicolumn{2}{|c|}{$\begin{array}{c}\text { Long-term Relief } \\
\text { Control vs. } \\
\text { Treatment }\end{array}$} & \multirow[t]{2}{*}{ Results } \\
\hline & & & & & & 3 Months (\%) & 6 Months (\%) & \\
\hline Riew et al (622) & P, RA, DB & 55 & LA, S & $1-4$ & 33 vs. 71 & 33 vs. 71 & 33 vs. 71 & $P$ \\
\hline Kraemer et al (624) & $\mathrm{P}, \mathrm{RA}, \mathrm{PC}, \mathrm{DB}$ & 49 & $\mathrm{~S}, \mathrm{NS}$ & N/A & $\mathrm{E}$ & E & $\mathrm{E}$ & $P$ \\
\hline Kraemer et al (624) & P, RA & 87 & LA, S & N/A & $\mathrm{E}$ & $\mathrm{E}$ & $\mathrm{E}$ & $P$ \\
\hline Shah et al (623) & $\mathrm{P}, \mathrm{PC}$ & 48 & LA, S & $1-4$ & 84 & 84 & 84 & $P$ \\
\hline Lutz et al (625) & $\mathrm{P}, \mathrm{C}$ & 69 & LA, S & $1-4$ & 79 & 79 & 79 & $P$ \\
\hline $\begin{array}{l}\text { Manchikanti et al } \\
\text { (553) }\end{array}$ & R, RA & 225 & S, LA & $1-10$ & 91 & 75 & 70 & $P$ \\
\hline $\begin{array}{l}\text { Bush and Hillier } \\
\text { (571) }\end{array}$ & $\mathrm{P}, \mathrm{C}$ & 68 & LA, S & $2-3$ & 93 & 93 & 93 & $P$ \\
\hline Kikuchi et al (415) & $\mathrm{R}$ & 332 & S, LA & N/A & N/A & N/A & 64 & $P$ \\
\hline
\end{tabular}

$\mathrm{P}=$ prospective $; \mathrm{R}=$ retrospective $\mathrm{C}=$ controlled $\mathrm{PC}=$ placebo controlled; $\mathrm{RA}=$ randomized $\mathrm{DB}=$ double-blind;

$\mathrm{LA}=$ local anesthetic $\mathrm{S}=$ steroids; $\mathrm{N} / \mathrm{A}=$ not available; $\mathrm{NS}=$ normal saline; $\mathrm{E}=$ effective $; P=$ positive; $\mathrm{VS}=$ versus

in the study, and any patient with more than two levels of disease. Progress was monitored using the NASS Outcome questionnaire and a specifically designed nerve root injection questionnaire. All subjects were assessed at baseline; at 2, 4, and 8 weeks post-injection; and again at 1 year. The primary outcome measure was whether patients underwent surgery; but pain, disability, patient satisfaction, and treatment expectations were also evaluated. Both groups of patients had similar demographic and clinical characteristics. They were randomly allocated to receive an injection of a corticosteroid plus a local anesthetic, or the anesthetic alone, in a double-blinded manner. All patients received injections under fluoroscopy, up to four over the course of the study. All patients had the option of choosing surgery or participating in the study. Each patient received one or more additional injections as randomized. Authors concluded that $71 \%$ of the patients studied with nerve root injections of corticosteroids avoided surgery, compared to $33 \%$ of control subjects. However, patients who opted not to have surgery showed greater improvement in terms of pain reduction, functional status improvement, and expectation of recovery than those who went on to have surgical intervention. The authors concluded that selective nerve root injection(s) of corticosteroids were efficacious in preventing typical spine surgery. They also speculated that selective nerve root injections might be effective because they provided more focal delivery of corticosteroids to the compressive nerves than other types of epidural injections. This study also showed that the first injection had the greatest impact on symptoms, with subsequent injections having less of an effect. The injections appear to provide benefit for patients with both acute and chronic complaints. However, it is also important to note that $33 \%$ of the patients in the local anesthetic injection group also avoided surgery.

Kramer et al (624), in a prospective, randomized, controlled trial, evaluated the role of lumbar epidural perineural injections. They included two controlled studies to evaluate single-shot, selective nerve root injection with a doubleneedle approach to the anterior epidural space of the lumbar spinal canal. The trial comprised two controlled studies on 182 patients. One study compared prospectively randomized results of patients with lumbar radicular syndromes: 47 received epidural perineural injections, 40 received conventional posterior epidural injections and, 46, as a control group, received paravertebral local anesthetic injection. Along with this, a second, prospective, doubleblind study compared the effect of epidural perineural injections with triamcinolone in 24 patients and normal saline in 25 patients. Epidural perineural injections were more effective than conventional posterior epidural injections. Both epidural groups had better results than the paravertebral local injection group. Epidural perineural injections with steroids utilizing $10 \mathrm{mg}$ of triamcinolone were more effective than saline alone. A systemic steroid effect was excluded by additional intramuscular steroid injections in the normal saline group. The authors con- 
cluded that in both studies, the single-shot epidural perineural injection is effective in the treatment of lumbar radicular pain.

Shah et al (623) compared the efficacy of fluoroscopically guided transforaminal epidurals for lumbar radiculopathy due to disc herniation with another group of patients who underwent trigger point injections in an office setting. Fifty patients were assessed with an average follow-up of 1.4 years. Patients who had documented lumbar disc herniation on MRI, greater than $50 \%$ of the total pain present in the leg and/or buttock, at least 6 weeks of symptoms, and who failed to improve with oral medications and rehabilitation were included. They excluded patients with history of previous spinal surgery. Patients were randomly divided into two groups. Group I with 25 patients with average age of 41.3 years, received an average of 1.7 fluoroscopically guided transforaminal injections combined with home lumbar stabilization program and a back cryobrace. Group II with 23 patients with average age of 42.4 years received an average of 1.6 saline trigger point injections combined with home lumbar stabilization program and a back cryobrace. At three months, the nonresponders in group II were crossed into group I. The outcomes consisted of patient satisfaction rated from poor to excellent, pain score, Rolland-Morris questionnaire, and distance from finger to floor in centimeters collected pre-, and 3 weeks, 6 weeks, 3 months, 6 months, and 1 year post-treatment. They defined a successful outcome as good or better satisfaction combined with greater than $50 \%$ reduction in pain score. The results showed that in Group I, average Rolland-Morris score was 8.8 pre- and 22.1 post-treatment, pain score was 8.8 pre- and 1.6 post-treatment, and distance from finger to floor was $69.6 \mathrm{~cm}$ pre- and $20.3 \mathrm{~cm}$ post-treatment. Overall, Group I had $84 \%$ successful outcome. Group II also showed significant improvement but only resulting in $48 \%$ successful outcome. For Group II, the average Rolland-Morris score was 9.6 pre- and 18.3 post-treatment, pain score of 9.4 pre- and 3.6 post-treatment, and distance from finger to floor was 64.8 pre- and 24.4 post-treatment. Thus, Group I had a significantly better outcome than Group II at 1.4 year average follow-up (P>0.05). They also reported that the nonresponders who crossed over from Group II to Group I experienced $67 \%$ successful outcome. They reported that presence of spondylolisthesis, in addition to disc herniation, was a negative prognostic factor for Group I, whereas symptom duration greater than six months was a negative prognostic factor for Group II patients.

Lutz et al (625) studied 69 patients in a prospective case series. They investigated the outcome of patients with lumbar herniated nucleus pulposus and radiculopathy using administration of fluoroscopically guided transforaminal epidural steroid injections. Patients were evaluated by an independent observer and were followed for an average period of 80 weeks, with a range of 28 to 144 weeks. Among the 69 patients, $75 \%$ of the patients had a successful long-term outcome, reporting at least a greater than $50 \%$ reduction between preinjection and postinjection pain scores, as well as an ability to return to or near their previous levels of functioning after 1.8 injections per patient (range, one to four injections). They concluded that fluoroscopic transforaminal epidural steroids are an effective nonsurgical treatment option for patients with lumbar herniated nucleus pulposus and radiculopathy in whom more conservative treatments are not effective.

Bush and Hillier (571) described the response of 68 patients to cervical epidural steroid injections with some of them undergoing transforaminal epidural injections if they failed non-fluoroscopically guided lateral approach at C7. Following the first blind cervical epidural injection, if significant improvement was not seen, a repeat injection was performed transforaminally with fluoroscopic guidance within one month. Similarly, a third injection was also performed if needed in the same manner as the second injection. Overall, an average of 2.5 injections per patients was required for adequate pain control; $93 \%$ of the patients were reported to have good pain relief lasting for seven months.

Weiner and Fraser (626) treated 28 patients with severe radiculopathy secondary to foraminal or extraforaminal herniation of lumbar disks. In these patients, the disk herniation was proven by imaging studies; and it failed to respond to rest and anti-inflammatory therapy, epidural injections, and physical therapy. The only remaining choice for these patients was surgical intervention due to the severity of pain and functional disability. The authors showed that 22 of the 28 patients improved dramatically, with sustained relief lasting an average of 3.4 years, with a range of 1 to 10 years. Further analysis showed that, of the 28 patients, three obtained no relief and subsequently underwent diskectomy; but three obtained immediate relief and relapsed within 6 months. In addition, one patient obtained minimal relief but was able to tolerate continuing symptoms; and seven patients received moderate relief that allowed them to return to most activities but with caution and occasional symptomatic treatment. Of the 28 patients, 14 had complete relief of their pain at follow-up that ranged from 1 to 10 years. 
Kikuchi et al (415) studied the therapeutic effect of transforaminal nerve root injections in 332 patients. They reported that this procedure not only had therapeutic effect but also had great diagnostic value in functional as well as morphological aspects. They reported that 22 of 45 patients with disk ruptures, 30 of 39 patients with spondylosis, and five of six patients with degenerative spondylolisthesis all experienced more than 6 months of pain relief and thus were able to avoid surgical intervention. Furthermore, they reported that, over the long term, relief was seen in $64 \%$ of these patients.

Manchikanti et al (553) compared the three routes of epidural steroid injections in the management of chronic low back pain. This retrospective evaluation included 225 patients, randomly derived from a total sample of $624 \mathrm{pa}-$ tients suffering with low back pain from a total of 972 patients referred for pain management. The evaluation was performed by an independent evaluator. The study design included three groups: Group I, which received interlaminar epidurals with a midline approach in the lateral position, with entry between L3/4 or L4/5 in nonsurgical patients and above the scar either at $\mathrm{L} 2 / 3$ or $\mathrm{L} 1 / 2$ in postsurgical patients, using a loss-of-resistance technique; Group II, which received caudal epidurals, the procedures being performed in prone position, under fluoroscopy, with confirmation of the position by injection of contrast; and Group III, which received transforaminal epidural corticosteroid injections, using either sacral or lumbar transforaminal technique under fluoroscopy. The results of the study showed that all three routes of administration of epidural corticosteroid administration were clinically effective, though administration by caudal and transforaminal routes was more successful in obtaining longer term relief. Further, this study also showed that the transforaminal injections were the ideal, as the most significant improvement was noted with the least expense compared to fluoroscopically directed caudal epidural, and to blind interlaminar epidural. This study showed significant relief, which was defined as greater than $50 \%$ per procedure for all patients in the study as $3.45+/-0.17$ weeks, $6.06+/-1.27$ weeks, and $7.69+/$ 1.20 weeks for blind intralaminar epidural, fluoroscopically directed caudal and transforaminal epidural injections, respectively.

Devulder (627) also studied transforaminal epidural injections, which he termed nerve root sleeve injections with corticosteroids; however, they were in combination with hyaluronidase. In a study of 20 patients with persistent pain after surgery, ranging from 1 to 9 years in duration and diagnosed as failed back surgery syndrome, they reported that $55 \%$ of the patients reported greater than $50 \%$ relief at 1 month, while $50 \%$ of the patients experienced continued relief after three months.

Slipman et al (628), in a retrospective analysis with independent clinical review, evaluated the role of therapeutic selective nerve root block in the nonsurgical treatment of atraumatic cervical spondylotic radicular pain. Of 20 subjects, 10 men and 10 women, with a mean age of 56.6 years and an average symptom duration of 5.8 months, were treated with an average of 2.2 therapeutic injections. They reported an overall good or excellent result in $60 \%$ of the patients, with significant reduction in pain scores, as well as significant reduction in medication usage.

Of the five prospective clinical trials available for evaluation of transforaminal epidural injections, three were considered of moderate quality (622-624) and the remaining two were considered of low quality $(571,625)$. In terms of the evaluation of the type and strength of efficacy evidence of transforaminal epidural injections is level II to level III, strong to moderate. Level II - strong is defined as evidence with research-based evidence with at least one properly designed randomized controlled trial of appropriate size and with 60 patients and high quality or multiple adequate scientific studies. Level III - moderate is defined as evidence obtained from well-designed trials without randomization, single group prepost, cohort, time series, or matched case controlled studies.

\section{Percutaneous Lysis of Epidural Adhesions}

Percutaneous epidural adhesiolysis, lysis of epidural adhesions, percutaneous neuroplasty, or epidural neurolysis is an interventional pain management technique that played an active role since its emergence during the latter part of 1980s in managing chronic intractable low back pain (632, 633). Postlumbar laminectomy syndrome or pain following operative procedures of the spine is a common entity in modern medicine (261-292). Ross and coworkers (267), in a study of the relationship between peridural scar evaluated by MRI and recurrent radicular pain after lumbar discectomy, showed that subjects with extensive peridural scarring were 3.2 times more likely to experience recurrent radicular pain. Park and Watanable (269) analyzed the frequency and location of lumbar and ventral dural adhesions in elderly cadavers, showing significant evidence of adhesions in $40 \%$ at L4/5 levels, in $36 \%$ at L5/S1 levels, and in $16 \%$ at L3/4 levels. Even though epidural adhesions are most commonly observed following surgical 
intervention of the spine, leakage of the disc material into the epidural space following an annular tear has also been reported to cause fibrocyte deposition and an inflammatory response that can subsequently result in the formation of epidural adhesions $(227,636,637)$. It has been presumed that inflammation and compression of nerve roots by epidural adhesions is the mechanism of persistent pain in patients. The causes of failed back surgery syndrome or postlumbar laminectomy syndrome are epidural scarring, arachnoiditis, recurrent disc herniation with neural encroachment, mechanical instability, and facet degeneration. While it is largely agreed that peridural scarring contributes to a considerable amount of morbidity and mortality following lumbar surgery, further surgery is not a solution, as results show disappointing success rates as low as $12 \%$ (280, 638). Further, epidural adhesions are not readily diagnosed by conventional studies such as myelography, CT, and MRI, even though modern technology has made significant improvements in this area (637). The epidural adhesions are best diagnosed by performing an epidurogram, which is most commonly performed via the caudal route, followed by other routes, including the lumbar interlaminar route, and thoracic and cervical interlaminar routes $(525,527,632-634,636,639-641)$. Epidural filling defects have also been shown in a significant number of patients with no history of prior surgery (525). While peridural scarring in itself is not painful, it can produce pain by "trapping" spinal nerves so that movement places tension on the inflamed nerves $(633,634,639)$. Kuslich and coworkers (153) reported that back pain was produced by stimulation of several lumbar tissues, even though the outer layer of the annulus fibrosus and posterior longitudinal ligament innervated by the sinuvertebral nerves were the most common tissues of origin.

Adhesiolysis of epidural scar tissue, followed by the injection of hypertonic saline, has been described by Racz and coworkers in multiple publications (632-635, 636, 639, 644, 645). The technique described by Racz and colleagues involved epidurography, adhesiolysis, and injection of hyaluronidase, bupivacaine, triamcinolone diacetate, and 10\% sodium chloride solution on day 1 , followed by injections of bupivacaine and hypertonic sodium chloride solution on days 2 and 3. Manchikanti and colleagues (632, 646, 649) modified the Racz protocol from a 3-day procedure to a 1-day procedure.

The purpose of percutaneous epidural lysis of adhesions is to eliminate deleterious effects of scar formation, which can physically prevent direct application of drugs to nerves or other tissues to treat chronic back pain. In addition, the goal of percutaneous lysis of epidural adhesions is to assure delivery of high concentrations of injected drugs to the target areas.

Clinical effectiveness of percutaneous adhesiolysis was evaluated in one randomized controlled trial $(647,648)$ and four retrospective evaluations (636, 646, 649, 650). Racz and colleagues (647), and Heavner and coworkers (648), studied percutaneous epidural adhesiolysis, with a

Table 13. Results of published reports of percutaneous lysis of lumbar epidural adhesions and hypertonic saline neurolysis for a single procedure

\begin{tabular}{|c|c|c|c|c|c|c|c|}
\hline \multirow[t]{2}{*}{ Author(s) } & \multirow{2}{*}{$\begin{array}{c}\text { Study } \\
\text { Characteristics }\end{array}$} & \multirow{2}{*}{$\begin{array}{c}\text { No. of } \\
\text { Patients }\end{array}$} & \multirow[t]{2}{*}{ Drugs Utilized } & \multirow{2}{*}{$\begin{array}{l}\text { No. of } \\
\text { Days of } \\
\text { Procedure }\end{array}$} & \multirow{2}{*}{$\begin{array}{c}\text { Initial Relief } \\
1-4 \\
\text { Weeks }\end{array}$} & \multicolumn{2}{|c|}{ Long-term Relief } \\
\hline & & & & & & 3 Months & 6 Months \\
\hline Heavner et al (648) & $\mathrm{P}, \mathrm{RA}, \mathrm{PC}$ & 59 & $\mathrm{~B}, \mathrm{~T}, \mathrm{H}, \mathrm{HS}, \mathrm{NS}$ & 3 & $83 \%$ & $49 \%$ & $43 \%$ \\
\hline $\begin{array}{l}\text { Racz and Holubec } \\
\text { (636) }\end{array}$ & $\mathrm{R}, \mathrm{RA}$ & 72 & $\mathrm{~B}, \mathrm{~T}, \mathrm{H}, \mathrm{HS}$ & 3 & $65 \%$ & $43 \%$ & $13 \%$ \\
\hline $\begin{array}{l}\text { Manchikanti et al } \\
\text { (646) }\end{array}$ & $\mathrm{R}, \mathrm{RA}$ & 103 & M, L, HS & 2 & $74 \%$ & $37 \%$ & $21 \%$ \\
\hline $\begin{array}{l}\text { Manchikanti et al } \\
(646)\end{array}$ & $\mathrm{R}, \mathrm{RA}$ & 129 & M, L, HS & 1 & $79 \%$ & $26 \%$ & $14 \%$ \\
\hline $\begin{array}{l}\text { Manchikanti et al } \\
\text { (649) }\end{array}$ & $\mathrm{R}$ & 60 & L, HS, CS & 1 & $100 \%$ & $25 \%$ & $10 \%$ \\
\hline Arthur et al (650) & $\mathrm{R}, \mathrm{RA}$ & 100 & $\mathrm{~L}, \mathrm{HS}, \mathrm{CS}, \mathrm{H}$ & 1 & $82 \%$ & NA & $14 \%$ \\
\hline
\end{tabular}


Table 14. Results of 1-year follow-up of patients following percutaneous lysis of lumbar epidural adhesions

\begin{tabular}{|c|c|c|c|c|c|c|c|}
\hline \multirow[t]{2}{*}{ Author(s) } & \multirow{2}{*}{$\begin{array}{c}\text { Study } \\
\text { Characteristics }\end{array}$} & \multirow{2}{*}{$\begin{array}{l}\text { No. of } \\
\text { Patients }\end{array}$} & \multirow{2}{*}{$\begin{array}{c}\text { No. of Days } \\
\text { of } \\
\text { Procedure }\end{array}$} & \multicolumn{4}{|c|}{ Patients with Significant Relief } \\
\hline & & & & 1 Month & 3 Months & 6 Months & 12 Months \\
\hline $\begin{array}{l}\text { Racz et al (647) and } \\
\text { Heavner et al (648) }\end{array}$ & $\mathrm{P}, \mathrm{C}, \mathrm{RA}$ & 59 & 3 & $83 \%$ & $49 \%$ & $43 \%$ & $49 \%$ \\
\hline Manchikanti et al (649) & $\mathrm{R}$ & 60 & 1 & $100 \%$ & $90 \%$ & $72 \%$ & $52 \%$ \\
\hline
\end{tabular}

$\mathrm{R}=$ retrospective $\mathrm{P}=$ prospective, $\mathrm{RA}=$ randomized $\mathrm{C}=$ controlled

prospective evaluation of $0.9 \%$ sodium chloride solution versus $10 \%$ sodium chloride solution with steroids, with prospective 1-year follow-up. They concluded that percutaneous epidural neuroplasty, as part of an overall pain management strategy, reduces pain in $25 \%$ or more of patients with radiculopathy plus low back pain refractory to conventional therapies. They also noted that the use of hypertonic saline and hyaluronidase may reduce the number of patients that require additional treatments. However, adhesiolysis was effective, even in the patients receiving normal saline. They also showed that the percent of patients requiring additional treatments during 1-year follow-up was approximately $70 \%$, at on average, around 70 days. This percentage was approximately $60 \%$ in patients receiving hypertonic saline, and $80 \%$ in patients receiving normal saline. Finally, Heavner and coworkers (648) concluded that the most significant finding of the study was that at 1-year follow-up, $49 \%$ of the patients had pain relief in the body area targeted for the lesionspecific therapy.

Manchikanti and coworkers (646), evaluating 232 patients, with modification of the Racz protocol from a 3-day procedure to a 2-day procedure and a 1-day procedure, showed significant pain relief lasting at least 1 month in $52 \%, 2$ months in $35 \%, 3$ months in $11 \%$, and 6 months in $7 \%$ of patients with the first injection; and with better results with the second injection. However, no significant differences were noted between 1-day, 2-day, or 3-day procedures.

Racz and Holubec (636), in their earliest publication, reported favorable results with good-to-excellent pain relief for up to one month in $65 \%$ of the patients, for one to three months in $43 \%$ of the patients, and for three to six months in $13 \%$ of the patients. Arthur and colleagues (650), in studying 100 patients, concluded that when hyaluronidase was added to the injected, $82 \%$ reported initial pain relief compared to $68 \%$ in those without the hyaluronidase. However, no difference was seen in long-term improvement (14\% vs $12 \%)$.

In a study evaluating the effectiveness of nonendoscopic adhesiolysis in postlumbar laminectomy syndrome in 60 patients, Manchikanti and colleagues (649) reported relief of $12 \pm 3.2$ weeks relief with the first procedure, whereas with the second procedure it was $13 \pm 2.9$ weeks using a modified 1-day adhesiolysis. This study also showed 1year relief in $52 \%$ of patients, with repeat procedures of $2.98 \pm 0.16$ over a 1 -year period per patient. Tables 11 and 12 show the results of published reports of nonendoscopic adhesiolysis and hypertonic neurolysis with their effectiveness or lack thereof.

In contrast to the above reports, Devulder and coworkers (642) concluded that epidurography might confirm epidural filling defects, but a better contrast spread, assuming scar lysis does not guarantee sustained pain relief, as filling defects were confirmed in $88 \%$ of the patients with epidurography; but significant pain relief was seen in only $33 \%$ of the patients at 1 month, $13 \%$ at 3 months, and $0 \%$ at 12 months. However, the problem with this study was that lysis of adhesions was not lesion specific. Consequently, the delivery of drugs was also nonspecific (651653).

The quality of evidence presented above includes one randomized clinical trial which is of moderate quality, followed by three retrospective trials, two of which were randomized (Tables 13 and 14). The type and strength of efficacy evidence is type III - moderate, defined as evidence obtained from well-designed trials without randomization, single group prepost, cohort, time series, or matched case controlled studies. 


\section{Epiduroscopy or Spinal Endoscopy}

Epidural lysis of adhesion and direct deposition of corticosteroids in the spinal canal are also achieved with a threedimensional view provided by epiduroscopy or spinal endoscopy.

Buurman (654) in 1931 pioneered direct visualization of the spinal canal and its contents. In 1985 Blomberg (655) of Sweden described a method of epiduroscopy. In 1991, Saberski and Kitahata (656) started using fiberoptic endoscopes for epiduroscopy. Heavner and colleagues (657) also reported in 1991 on endoscopic evaluation of the epidural and subarachnoid spaces in animals and human cadavers. By 1996, epidural spinal canal endoscopy was used frequently for delivery of epidural steroid medication (658-663).

There have been a few retrospective analyses performed to evaluate the efficiency of spinal endoscopy; however, there are no randomized, controlled trials $(650,661,663)$.

Manchikanti et al (650), in a study evaluating the effectiveness endoscopic adhesiolysis in post lumbar laminectomy syndrome in 60 patients, showed that $100 \%$ of the patients reported significant pain relief at one month, whereas $75 \%$ reported significant relief at three months; $40 \%$ reported at six months, and $22 \%$ reported at 12 months. It was concluded that endoscopic adhesiolysis with administration of corticosteroids is a safe and possibly cost-effective technique for relief of chronic intractable pain failing to respond to other modalities of treatments.

Manchikanti et al (661) studied the value and safety of epidural endoscopic adhesiolysis. In this retrospective evaluation on 85 consecutive patients undergoing 112 epidural endoscopic procedures. They reported significant pain relief in $100 \%$ of the patients, initially decreasing to $94 \%$ at one to two months, to $77 \%$ at two to three months, to $52 \%$ at three to six months, to $21 \%$ at six to twelve months, and to $7 \%$ after 12 months. They concluded that epidural endoscopy with adhesiolysis is a relatively safe and possibly cost-effective technique in the management of chronic refractory low back pain.

Saberski (663), in a retrospective analysis of spinal endoscopy and laminectomy, reported outcome data in a pilot study. This pilot study included two groups of patients, Group I, with 22 patients treated via spinal endoscopy; and Group II with 13 patients treated via laminectomy. After spinal canal endoscopy, only $32 \%$ of Group I patients were continued on opioid medication; whereas $92 \%$ of Group II patients were continued on opioid medication after laminectomy. In addition, $72 \%$ from the spinal canal endoscopy group and only $28 \%$ from the laminectomy group returned to work. He concluded that this study suggested remarkable differences in outcomes when comparing patients who underwent spinal canal endoscopy to a similar population who underwent lumbar laminectomy. Based on the above, the type and strength of efficacy evidence analysis places spinal endoscopy into type IV-limited, which is defined as evidence from well designed non experimental studies from more than one center or research group: but this evidence is also complemented by clinical experience.

\section{Intradiscal Electrothermal Annuloplasty}

Primary discogenic pain is a common entity with or without internal disc disruption and is responsible for chronic low back pain in approximately 39\% of patients (174). This is in contrast to disc herniation, which is seen in a small number of patients ranging from $4 \%$ to $6 \%(173$, 222, 223, 255-260). Intradiscal electrothermal annuloplasty (IDET) is a minimally invasive treatment for chronic discogenic low back pain that is an alternative to interbody fusion surgery (664). Application of thermal energy to the disc alters collagen structure and may perform a functional deafferentation on the disc. The technique of intradiscal electrothermal annuloplasty utilizes this principle to treat patients with intractable low back pain. Multiple investigators have studied the effectiveness of intradiscal thermal annuloplasty (664-674). However, only one published study included a control group (669), whereas another study incorporated results of a multicenter cohort study (670) and the remaining five studies were descriptive in nature $(664,666-668,674)$. Apart from these, there were presentations at multiple meetings, some of which are listed here; however, it appears many of them included the same patients but were presented repeatedly.

Karasek and Bogduk (669) studied 53 patients with back pain determined by CT discography to be due to internal disc disruption. The outcomes of 35 patients treated with IDET were compared with those of a convenience sample of 17 patients treated with a physical rehabilitation program, by using VAS scores, use of analgesics, and return to work as measures. They reported that, at 3 months, only one control patient obtained any significant degree of relief of pain, compared with 23 in the index group. Re- 
lief of pain was sustained at 6 and 12 months and was associated with improvement in disability, reduced drug use and a return to work rate of $53 \%$. They concluded that in carefully selected cases, IDET can eliminate or dramatically reduce the pain of internal disc disruption in a substantial proportion of patients and appears to be superior to conventional conservative care for internal disc disruption, with a success rate as low as $23 \%$ or as high as $60 \%$, with confidence intervals of $\pm 16 \%$.

Wetzel et al (670) reported the preliminary results of a multicenter prospective cohort study of intradiscal electrothermal annuloplasty to treat discogenic low back pain. The study group included patients from centers in Chicago, Dallas, Plano, Roseland, and Syracuse. A total of 78 patients were entered in the intent-to-treat group. The inclusion criteria were: complaint of predominantly low back pain, persistence of symptoms for greater than 3 months, and failure of at least 6 weeks of conservative care. Exclusion criteria were: sequestered disc herniation, greater than two levels of symptomatic degenerative disc disease, spinal stenosis, spondylolisthesis or previous surgery at the treated level. Patients were reevaluated at 1 month, 3 months, 6 months, and 1 year. There were eight withdrawals from the study. Seventy patients completed the study, with five failures, four who went to fusion, and one who received a second IDET. In all, 93 levels were treated in 65 patients. Twenty-nine patients underwent two-level treatment and thirty-five underwent one-level treatment. They reported significant improvement in VAS scores, bodily pain, physical function, social function, reported health transitions, physical health and pain, treatment expectation, patient satisfaction and pain disability at 3,6, and 12 months. Significant changes in a greater work ability were also seen at one year, along with improvement in ability to sit and walk. The initial failure rate was $6.9 \%$; however, the authors have not described success rate with the number of patients.

Saal and Saal (667), in a prospective case series, reviewed outcomes of 62 patients with unremitting chronic discogenic low back pain who were faced with a choice of long term pain management or fusion surgery. They treated these patients with IDET, with a mean follow-up of 16 months, and mean preoperative duration of symptoms of 60 months. They reported improvement in $70 \%$ of the patients, not only with physical pain but also with return to work. Saal and Saal (674) also reported their findings with a 2-year follow up. Outcome scores at 1-year follow up were not statistically different from outcome scores as- sessed at 2-year follow-up.

Singh (668) published a preliminary report evaluating 23 patients at 6 months with improvement in $70 \%$ of the patients. Derby et al (666) reported their findings of IDET in a 1-year pilot outcome study with 32 patients. They reported that $63 \%$ of the patients had a favorable outcome, with no change in outcome measures at 6-month and 12month follow-ups. Derby and O'Neill (671) evaluated the effects of IDET on referred leg pain, reporting significant relief of referred leg pain. Even though the mechanism of relief is unknown, a reduction in the chemical sensitivity and reduction in inflammatory chemical substances in the outer annulus and adjacent epidural space are proposed to explain the results. Liu et al (675) attempted to identify factors associated with favorable outcomes in 50 patients treated with IDET. They reported overall favorable clinical results in $60 \%$ of the patients. They also reported that the results were less favorable with time decreasing to $43 \%$ at 12 months and $33 \%$ at 18 months. They concluded that the IDET procedure for degenerative discs achieved clinically favorable results in $60 \%$ of the patients. However, these results can be substantially improved by proper patient selection and careful attention to correct catheter placement. Predictive clinical factors included: age less than 40; nonsmoker status; female sex; symptoms of less than 4 years; modified Dallas type 1, 2, 3 annular tears; and perfect spine catheter placement along the entire posterior annulus. Maurer et al (673) investigated 36 consecutive patients in a prospective case series who underwent IDET. They reported that at six months, $94 \%$ of patients had a mean decrease of four points on VAS. Functional scales (sitting, standing, walking) increased on average $75 \%$. Lee et al (675) evaluated the stability of the spine after intradiscal electrothermal therapy. This was an in vitro study to analyze whether or not there was any significant change in human cadaveric disc stability after IDET. Preliminary results of this study suggested that there is no significant difference in spine segmental stability before and after treatment with IDET in vitro.

Based on the above reports which included two prospective evaluations and multiple observational studies, in terms of type and strength of efficacy evidence is type III - moderate, which is evidence from well-designed trials without randomization, single group prepost, cohort, time series, or matched case controlled studies complimented from well-designed non-experimental studies and also opinions 
of respected authorities.

\section{Sympathetic Blocks}

Management options for reflex sympathetic dystrophy (RSD) and causalgia, also known as complex regional pain syndrome (CRPS) I and II, include sympathetic blocks utilizing regional anesthetic techniques and radiofrequency thermoneurolysis or neuromodulation with spinal cord stimulation or peripheral nerve stimulation. Radiofrequency neurolysis is an extension of a continuous regional sympathetic block or neurolytic block providing long term relief with added safety. Consideration of sympathetic blocks is to facilitate management of CRPS with analgesia commensurate with a program of functional restoration and sympatholysis to provide unequivocal evidence of sympathetically maintained pain. Once it is established that sympatholysis is effective in relieving not only the burning dysesthesia but also allodynia or hyperalgesia, it is important to repeat the procedure to determine whether an increasing duration of effect can be expected in any particular patient. If this is the case, these individual blocks may be all that are necessary to enable a patient to regain function. When sympatholysis completely relieves the symptoms and facilitates exercise therapy but is limited to its duration of effect, it is appropriate to consider a prolonged block using radiofrequency neurolysis. Radiofrequency has been described for lesioning of the cervical sympathetic chain, thoracic sympathetic chain, and lumbar sympathetic chain, in cases of CRPS I and II, as well as for neuropathic pain.

Multiple authors have described their experience with local anesthetic blockade, as well as radiofrequency neurolysis; however, there are no large scale either prospective or retrospective case study series (676-684). However, neurolytic celiac plexus block for treatment of cancer pain has received significant attention in the literature (685). Eisenberg et al (685) performed a meta-analysis of the efficacy and safety of neurolytic celiac plexus block for cancer pain. They reviewed a total of 24 papers which met inclusion criteria with two of them being randomized, controlled trials $(686,687)$. One was prospective $(688)$, and the remaining 21 were uncontrolled, retrospective studies (685). Both randomized, controlled trials $(686,687)$ showed positive results. Eisenberg et al (685) concluded that short-term success rate of neurolytic celiac plexus block is approximately $90 \%$, regardless of the underlying type of cancer. The data analyzed in this review suggested that neurolytic celiac plexus blocks can at least provide analgesia in addition to that achieved by opioids, and can re- duce their consumption $(685,689,690)$.

There is no significant evidence in the literature in the form of controlled trials for evaluation and management of sympathetically maintained pain either with local anesthetic blocks or neurolytic blocks, including radiofrequency thermoneurolysis. However, there is moderate evidence for neurolytic celiac plexus block for the treatment of cancer pain. Based on this, type and strength of efficacy evidence is level IV-limited, which is defined as the evidence from non-experimental studies from more than one center or research group.

\section{Trigger Point Injections}

Trigger point injections are probably the most extensively used modality of treatment, not only by interventional pain physicians, but all providers managing pain. Myofascial pain syndrome is a regional muscle pain disorder accompanied by trigger points. It has been described as a common phenomenon in multiple regions, including the spine (186-189, 202-209). Myofascial trigger points are small, circumscribed, hyperirritable foci in muscles and fascia, often found within a firm or taut band of skeletal muscle. In contrast, nonmyofascial trigger points may also occur in ligaments, tendons, joint capsule, skin, and periosteum (202). Trigger points assist in the proper diagnosis of myofascial pain syndrome, Simons (189) proposed major and minor criteria that should be met. The clinical criteria to establish a diagnosis of myofascial pain syndrome include five major criteria requiring all five to be present:

1. Regional pain complaint,

2. Pain complaint or altered sensation in the expected distribution of referred pain from a trigger point,

3. Taut band palpable in an accessible muscle,

4. Exquisite tenderness at one point along the length of the taut band, and some degree of restricted range of motion, when measurable.

Minor criteria of which only one of the three is required include:

1. Reproduction of clinical pain complaint,

2. Altered sensation, by pressure on the tender spot,

3. Local response elicited by snapping palpation at the tender spot or by needle insertion into the tender spot, and

4. Pain alleviated by elongating (stretching) the muscle or by injecting the tender spot. 
Even though there is a substantial amount of anecdotal evidence, there is no controlled prevalence data on the prevalence of myofascial pain. The authors exploring the role of trigger points and myofascial pain and whiplash injuries believe that the theory of trigger points lacks demonstrated internal validity. Formal studies also have shown that myofascial experts have difficulty in agreeing as to the presence of a trigger point, which is the cardinal feature of regional myofascial pain syndrome. In addition to this, it has been shown that topographically, trigger points of the neck overlay the cervical facet joints, and it has been reported that pain patterns of cervical trigger points are identical to those of referred pain from the facet joints. The same theories can be extrapolated to the lumbar spine.

The literature describing effectiveness of trigger point injections is enormous. There were seven controlled studies (203-209) along with numerous observational studies.

Collee et al (205), in a double-blind, randomized evaluation of local injection therapy of iliac crest pain syndrome and low back pain, studied the effectiveness of a single local injection of $5 \mathrm{~mL}$ of lignocaine, $0.5 \%$, with $5 \mathrm{~mL}$ isotonic saline in 41 patients. The results showed that in the local anesthetic group, $52 \%$ of the patients improved and in the saline group, only $30 \%$ improved. The data demonstrated an effect of the local injection with lignocaine that is somewhat larger than an injection with saline, which also has some beneficial effect. The difference was not consistent across all the settings (rheumatology practice vs general practice).

Bourne (206) compared corticosteroid - lignocaine injections with lignocaine alone in a trial of 57 patients suffering from chronic back pain. The results showed that corticosteroid - lignocaine mixture gave excellent results in $80 \%$ of 30 patients treated with the mixture and in only $16 \%$ of 19 patients treated with lignocaine alone.

Hamerhoff et al (207) compared bupivacaine, etidocaine, and saline for trigger point therapy in a randomized doubleblind crossover study. They reported increased relief with local anesthetic as compared with normal saline.

Fine et al (208) evaluated the effects of myofascial trigger point injections, they reported pain relief in all subjects with the injection of $0.25 \%$ bupivacaine injection along with improvement in range of motion in those subjects who initially demonstrated the limitation of movement. They also showed that the relief achieved with trigger point injections was reversed with naloxone but not placebo.
Jaeger and Shootsky (209) in a double-blind study evaluated the effect of dry-needling, saline, procaine, and placebo. They concluded that the use of saline or local anesthetic appears to be more effective than dry-needling or placebo.

In a controlled double-blind evaluation of the comparison of mepivacaine injection versus saline injection for myofascial pain. Frost et al (203) studied 28 patients with acute, localized muscle pain by injecting four local injections of mepivacaine, $0.5 \%$, in 28 patients, and local injection of an equal volume of normal saline in 25 patients. The group receiving saline tended to have more relief of pain, especially after the first injection. The results show that pain relief is not due merely to the local anesthetic. The study raises questions about the mechanism by which local injections into muscle relieve pain, since there is the possibility that a similar effect might also be achieved by merely inserting a needle into the trigger point. Normal saline is considered to be a more appropriate fluid for injection therapy than local anesthetic since it is less likely to produce side-effects. The positive aspects of this study include its inclusion of neck, shoulder, lumbar, and gluteal myofascial pain syndromes. The negative aspects include that normal saline was more effective than local anesthetic injection.

Garvey et al (204), in a prospective, randomized, doubleblind evaluation of trigger point injection therapy for low back pain, evaluated 63 individuals with low back strain. Patients with nonradiating low back pain, with normal neurological examination, without sciatic tension signs, and with negative radiological evaluation and patients who failed two months of conservative treatment were included. Injection therapy was of four different types: lidocaine, lidocaine combined with a steroid, acupuncture, and vapocoolant spray with acupressure. The results showed that noninjection therapy was effective in $63 \%$ of the patients whereas injection therapy was effective in only $42 \%$ of the patients. Thus, this study showed that trigger point therapy seems to be useful in the treatment of low back strain, but the injection substance apparently is not the critical factor, since direct mechanical stimulus to the trigger point seems to give symptomatic relief equal to that of treatment with various types of injected medication.

In terms of the quality of evidence presented with trigger point injection, the results were positive in five of the seven controlled studies $(205,209)$. Based on the above, type and strength of efficacy evidence is level III to level IV moderate to limited. Level III - moderate is defined as 
evidence from well-designed trials without randomization, single group pre-post, cohort, time series, or matched case controlled studies. Level IV- limited is defined as evidence obtained from well-designed non-experimental studies from more than one center or research group. In addition, there is overwhelming support from respected authorities along with clinical evidence and descriptive studies in support of judicious use of trigger point injections.

\section{Spinal Cord Stimulation}

Spinal cord stimulation uses electrical stimulation of spinal cord to control pain. There are multiple theories regarding how this effect causes pain control, but the exact mechanism is still controversial and may be a result of more than one of these mechanisms. In the United States, the primary indications for spinal cord stimulation are failed back surgery syndrome $(691,692)$, and both sympathetically maintained and sympathetically independent pain of complex regional pain syndrome (693-696). However, in Europe, most interest in spinal cord stimulation has been in the treatment of chronic intractable angina and pain and disability due to peripheral vascular disease (697-701). Spinal cord stimulation, for the clinical control of pain, was first introduced in 1967 by Shealy et al (702), in response to the publication of the gate control theory of pain by Melzack and Wall in 1965 (703).

In the field of spinal cord stimulation (SCS), as with other interventional techniques in chronic pain management, there are numerous retrospective studies that promote the efficacy of spinal cord stimulation, showing approximately $60 \%$ efficacy that lasts approximately two years $(691,704-$ 710). Wetzel et al (704) reviewed the current literature regarding the treatment of chronic pain in failed back surgery patients with spinal cord stimulation. Turner et al (69) also reviewed the literature on spinal cord stimulation in chronic low back pain in an attempt to perform a meta-analysis, concluding that this was not possible based on the characteristics of the literature. They analyzed 39 reports, "all case studies", concluding that $50 \%$ to $60 \%$ of patients with failed back surgery syndrome reported greater than $50 \%$ pain relief with the use of spinal cord stimulation.

North et al (711), in a prospective study, randomized 27 patients into repeat laminectomy or spinal cord stimulation groups. Even though this is not quite a similar treatment, the initial results were published after a 6-month follow-up; crossover between the groups was permitted. In this study, there was a significantly higher crossover rate from repeat laminectomy to spinal cord stimulation $(67 \%)$ than vice versa (17\%). Multiple observational studies in postlumbar laminectomy syndrome reported $25 \%$ to $76 \%$ pain relief at various intervals $(706-710,712,713)$.

In a prospective, multicenter study of spinal cord stimulation, Burchiel et al (697) demonstrated its effectiveness in the management of chronic low back and extremity pain. The permanent stimulating system was implanted in 182 patients. They reported at least $50 \%$ pain relief in $55 \%$ of the patients at 1-year follow-up.

Kemler et al (695) evaluated spinal cord stimulation in patients with chronic reflex sympathetic dystrophy utilizing a randomized trial involving patients who had had reflex sympathetic dystrophy for at least 6 months. Thirtysix patients were assigned to receive treatment with spinal cord stimulation plus physical therapy, and 18 were assigned to receive physical therapy alone. The health-related quality of life improved in the 24 patients who actually underwent implantation of a spinal cord stimulator. Of the 24 patients, 6 had complications that required additional procedures, including removal of the device in one patient. Thus, at 6 months, spinal cord stimulation was successful in 20 of 36 patients $(56 \%)$; however, since only 24 patients received spinal cord stimulation, this is 20 out of 24 patients with an $80 \%$ success rate.

Tesfaye et al (714) evaluated spinal cord stimulation for painful diabetic neuropathy in 10 patients who had not responded to conventional treatment. The electrode was implanted in the thoracic/lumbar epidural space. Immediate neuropathic pain relief after connecting the electrode was measured using a VAS, and exercise tolerance was assessed on a treadmill. Eight patients had statistically significant pain relief with an electrical stimulator, and the system was made permanent. Seven of these eight had statistically significant relief of pain at three months, and this relief was sustained in six patients until the end of the study at 14 months. These six patients used the stimulator as the sole treatment for their pain with improvement in their exercise tolerance. They claimed that electrical spinal cord stimulation offers a new and effective way of relieving chronic diabetic neuropathic pain, with improvement in exercise tolerance, in patients with neuropathic pain who do not respond to conventional treatment.

In addition to the declining success rate, complications also are common (713). These were predominantly electrode related problems i.e., migration, fracture, etc. Infection was less common, even though it was reported in $5 \%$ of 
the patients in 20 trials (713). Many new indications and techniques have evolved for SCS over the last several years including dual lead systems, retrograde cannulation, and transacral stimulation for pelvic pain.

Spinal cord stimulation is an invasive interventional surgical procedure. The difficulty of randomized clinical trials in such situations is well recognized. There were three prospective studies evaluating effectiveness of spinal cord stimulation in postlumbar laminectomy syndrome (711), reflex sympathetic dystrophy (695), and diabetic neuropathy (714). In addition, there have been numerous observational studies (691-694, 706-710, 712, 713). Based on the above reports the evaluation of the type and strength of efficacy evidence is level III - moderate. Level III - moderate evidence is defined as evidence obtained from welldesigned trials without randomization, single group prepost, cohort, time series, or matched case controlled studies.

\section{Implantable Intrathecal Drug Administration Systems}

Despite continued debate, chronic opioid therapy in the treatment of persistent pain of non cancer origin has gained broad acceptance (715-718), in addition to established chronic opioid therapy in cancer pain. The development of acceptable drug administration systems has been met with both enthusiasm (719) and controversy (720). Even though various guidelines have been proposed (721), much of the information is yet to come out or be absorbed about the long term effects of intrathecal opioid therapy. It appears that there is an increasingly large number of patients who have undergone intrathecal therapy for more than two years (722). Results indicating good to excellent outcome in nearly $70 \%$ of patient population which would have been considered quite refractory to standard types of management have been reported (721, 723-725).

Willis and Doleys (715), in a retrospective evaluation of 29 consecutive patients with a follow up duration of 31 months reported an average $63 \%$ improvement in pain, $46 \%$ improvement in activity level, and 54\% improvement in ease of performing activities. Other results were of Doleys et al (723), with $61 \%$ relief; Paice et al (726), also with $61 \%$ relief, though in a large, retrospective, multicenter survey. In other studies, Tutak and Doleys (727) reported a good or excellent outcome in $78 \%$ of the patients, Kremes and Lanning (719) reported good or excellent outcome in $81 \%$ of patients, and others $(724,725)$ at $70 \%$.

Other drugs also have been utilized in implantable sys- tems. Hilten et al (728) studied intrathecal baclofen for the treatment of dystonia in patients with reflex sympathetic dystrophy. They performed a double-blind, randomized, controlled, crossover of bolus intrathecal injections of 25,50 , and $75 \mathrm{mg}$ of baclofen in placebo. The results showed that in six women, bolus injections of 50 and 75 $\mathrm{mg}$ of Baclofen resulted in complete or partial resolution of focal dystonia of the hands but little improvement in dystonia of the legs. During continuous therapy, three women regained normal hand function and two of these three women regained the ability to walk. In one woman who received continuous therapy, the pain and violent jerks disappeared and the dystonic posturing of the arm decreased. In two women, the spasms and restlessness of the legs decreased, without any change in the dystonia. They concluded that in some patients, the dystonia associated with reflex sympathetic dystrophy responded markedly to intrathecal baclofen. Even though this was a double-blind, randomized, controlled, crossover trial, it included only a total of seven patients; but this probably is the best evidence available for this type of therapy in a randomized controlled trial.

Avellino and Loeser (729) also studied intrathecal baclofen for the treatment of intractable spasticity of the spine or brain etiology in a retrospective review of 62 consecutive adult patients who underwent placement of a programmable pump. They concluded that, intrathecal baclofen is an effective strategy for the relief of medically intractable spasticity of spine or brain etiology.

Intrathecal drug delivery system is an invasive surgical procedure. Again, this is met with difficulties with evaluation in a randomized clinical trial considering the various difficulties of a randomized clinical trial in such a situation along with the presence of one double-blind randomized controlled crossover study for baclofen and an enormous amount of evidence from observational studies. In consideration of multitude of factors, it is determined that, the evaluation of type and strength of efficacy evidence for intrathecal implantable drug delivery systems is level III - moderate. Level III - moderate is defined as evidence obtained from well-designed trials without randomization, single group pre-post, cohort, time series, or matched case controlled studies.

\section{Complications}

The most common and worrisome complications of interventional techniques are two-fold. These include complications related to a technique of an interventional proce- 
dure with placement of either the needle and/or catheter, and complications related to the administration of various drugs. Complications include dural puncture, spinal cord trauma, and infection.

Accidental dural puncture, subdural injection, neural trauma, injury to the spinal cord, and hematoma formation have been described. The incidence of dural puncture following lumbar epidural injections has been reported as $0.33 \%$ (60) and $0.25 \%$ after cervical epidural injections (730). Subdural intracranial air was also reported following epidural injections $(731,732)$. In addition, lumbar puncture has been reported following facet joint injections and sympathetic blocks (733-735). When C3/4, C4/5, or C5/6 facet joint blocks, the phrenic nerve may be compromised, especially if a large volume of local anesthetic is employed. This is also a complication of sympathetic block in the cervical spine. Spinal cord trauma, spinal cord or epidural hematoma formation is a catastrophic complication rarely seen following the interventional procedures in the cervical spine, thoracic spine or upper lumbar spine $(60,538-542,736-738)$. It has been suggested to perform interventional procedures with placement of a needle only in an awake patient and in the cervical spine by limiting the midline injection to be performed only at C7/T1 except in rare circumstances (538-542). However, unfortunately, it has been reported that even an awake patient may not be able to detect spinal cord puncture (739). Injection of neurolytic solutions or placement of radiofrequency needle into the spinal cord could lead to disastrous complications.

Infectious complications include epidural abscess and bacterial meningitis (740-756). However, iatrogenic spinal epidural abscess (757) and iatrogenic mycobacterium infection after an epidural injection was also reported (758). Discitis is considered as a principle complication of cervical discography which is seen less frequently following lumbar discography in approximately $0.1 \%$ to $1 \%$ of the patients $(759,760)$. Other complications include inadvertent subdural injection of local anesthetic and steroids (761, 762), development of complex regional pain syndrome (763), chemical meningism (764), lightheadedness, flushing, sweating, nausea, hypotension, syncope, pain at the injection site, and nonpostural headache (60). Retinal hemorrhage also has been associated with rapid injection of large volumes of caudal steroid injections (765).

Side-effects related to the administration of steroids are generally attributed either to the chemistry or to the pharmacology of the steroids. The major theoretical compli- cations of corticosteroid administration include suppression of pituitary-adrenal axis, hypercorticism, Cushing's syndrome, osteoporosis, avascular necrosis of bone, steroid myopathy, epidural lipomatosis, weight gain, fluid retention, and hyperglycemia (766-774). However, Manchikanti et al (775) in evaluating the effect of neuraxial steroids on weight and bone mass density showed no significant difference in patients undergoing various types of interventional techniques with or without steroids. Catheter shearing and inadvertent injection of hypertonic saline into the subarachnoid space as well as complications of hypertonic saline injection also have been described $(632,776-783)$. The most commonly used steroids in neural blockade in the United States, methylprednisolone acetate, triamcinolone acetonide, and betamethasone acetate and phosphate mixture have been shown to be safe at epidural therapeutic doses in both clinical and experimental studies (784-790).

Potential side-effects with radiofrequency denervation include painful cutaneous dysesthesias, increased pain due to neuritis or neurogenic inflammation, anesthesia dolorosa, cutaneous hyperesthesia, pneumothorax, and deafferentation pain, and finally inadvertent lesioning of the spinal cord and its contents (791).

Complications related to IDET, spinal cord stimulation, and intrathecal morphine implantation include various technical complications described above, other complications related to surgical technique itself, and fracture of the electrodes, shearing of the catheter, and complications related to long-term implantables.

\section{DELIVERY OF INTERVENTIONAL TECHNOLOGY}

\section{Dosage, Frequency, And Number of Blocks}

There is no consensus among the interventional pain management specialists with regards to type, dosage, frequency, total number of injections, or other interventions $(31,33$, 41-62, 338, 339, 607, 608, 792). Yet significant attention in the literature seems to be focused on the complications attributed to the use of epidural steroids in the entire arena of interventional pain management. Thus, various limitations of interventional techniques, specifically neural blockade, have arisen from basically false impressions. Based on the available literature and scientific application, the most commonly used formulations of long-acting steroids, which include methylprednisolone (DepoMedrol ${ }^{\circledR}$ ), triamcinolone diacetate (Aristocort ${ }^{\circledR}$ ) triamcinolone acetonide 
Table 15. Pharmacologic profile of commonly used steroids

\begin{tabular}{llccccccc}
\hline Name of the Drug & $\begin{array}{l}\text { Equivalent } \\
\text { Dose }\end{array}$ & $\begin{array}{l}\text { Epidural } \\
\text { Dose }\end{array}$ & $\begin{array}{l}\text { Anti- } \\
\text { inflammatory } \\
\text { Potency }\end{array}$ & $\begin{array}{l}\text { Sodium } \\
\text { Retention } \\
\text { Capacity }\end{array}$ & $\begin{array}{l}\text { Duration of Adrenal Suppression } \\
\text { IM }\end{array}$ & $\begin{array}{l}\text { Single } \\
\text { Epidural }\end{array}$ & $\begin{array}{l}\text { Three } \\
\text { Epidurals }\end{array}$ \\
\hline $\begin{array}{l}\text { Triamcinolone acetonide } \\
\text { (Kenalog) }\end{array}$ & $4 \mathrm{mg}$ & $40-80 \mathrm{mg}$ & 5 & 0 & $2-6$ weeks & N/A & $2-3$ months \\
$\begin{array}{l}\text { Betamethasone } \\
\text { (Celestone Soluspan) }\end{array}$ & $0.6 \mathrm{mg}$ & $6-12 \mathrm{mg}$ & 25 & 0 & $1-2$ weeks & N/A & N/A \\
$\begin{array}{l}\text { Triamcinolone diacetate } \\
\text { (Aristocort) }\end{array}$ & $4 \mathrm{mg}$ & $40-80 \mathrm{mg}$ & 5 & 0 & $1-2$ weeks & $1-5$ weeks & N/A \\
$\begin{array}{l}\text { Methylprednisolone acetate } \\
\text { (Depo-Medrol) }\end{array}$ & $4 \mathrm{mg}$ & $40-80 \mathrm{mg}$ & 5 & 0.5 & $1-6$ weeks & $1-3$ weeks & N/A \\
\hline
\end{tabular}

Reproduced with permission from Manchikanti $(338,339)$ IM = Intramuscular; N/A = Not Available

$\left(\right.$ Kenalog $\left.{ }^{\circledR}\right)$, and betamethasone acetate and phosphate mixture (Celestone Soluspan ${ }^{\circledR}$ ) appear to be safe and effective (Table 15) (41-62, 338, 339, 607, 608, 784-791). Based on the present literature, it appears that if repeated within two weeks, betamethasone probably would be the best in avoiding side effects; whereas if treatment is carried out at six-week intervals or longer, any one of the four formulations will be safe and effective.

Frequency and total number of injections or interventions are a key issue, although controversial and rarely addressed. Some authors recommend one injection for diagnostic as well as therapeutic purposes; others advocate three injections in a series irrespective of the patient's progress or lack thereof; still others suggest three injections followed by a repeat course of three injections after 3-, 6-, or 12month intervals; and, finally, there are some who propose an unlimited number of injections with no established goals or parameters. Limitation of $3 \mathrm{mg} / \mathrm{kg}$ of body weight of steroid or $210 \mathrm{mg}$ per year in an average person and a lifetime dose of $420 \mathrm{mg}$ of steroid, equivalent to methylprednisolone also have been advocated. While some investigators recommend one injection and do not repeat if there has been no response to the first, others recommend one or two more injections in the absence of response to the first injection. Some authors have reported good pain relief in previously unresponsive patients after an additional one or two injections. Similarly, some have believed that more than three injections do not result in additional improvement (572), whereas, others have reported the use of 6 to 10 injections if they are of benefit, however not to exceed 3 if they are not beneficial $(607,608)$. Such descriptions for other interventional techniques have been extrapolated from the limitations described for epidural steroid injections, even though there is no scientific basis or justification for such an extrapolation, as the techniques and type and dosage drugs are vastly different. It also has been shown in a multitude of publications that relief following multiple injections or interventions demonstrated a staircase-type phenomenon, even though it reached a plateau after three to four interventions.

\section{Facet Joint Injections:}

- In the diagnostic or stabilization phase, a patient may receive injections at intervals of no sooner than one week and preferably two weeks.

In the treatment or therapeutic phase (after the stabilization is completed), the frequency should be two months or longer between each injection provided that at least $>50 \%$ relief is obtained for six weeks. However, if the neural blockade is applied for different regions, they can be performed at intervals of no sooner than 1 week and preferably two weeks for most type of blocks. The therapeutic frequency must remain at least two months for each region.

- In the diagnostic or stabilization phase, the number of injections should be limited to no more than four times.

In the treatment or therapeutic phase, the interventional procedures should be repeated only as necessary judging by the medical necessity criteria and these should be limited to a maximum of 
six times for local anesthetic and steroid blocks for a period of one year.

- Under unusual circumstances with a recurrent injury or cervicogenic headache blocks may be repeated at intervals of six weeks after stabilization in the treatment phase.

\section{Medial Branch Neurolysis:}

- $\quad$ The frequency should be three months or longer between each neurolytic procedure provided that at least $>50 \%$ relief is obtained for 10 weeks. However, if the neural blockade is applied for different regions, they can be performed at intervals of no sooner than one week and preferably two weeks for most type of blocks. The therapeutic frequency for neurolytic blocks must remain at three months for each region.

- $\quad$ Neurolytic procedures should be repeated only as necessary judging by the medical necessity criteria and these should be limited to a maximum of four times for a period of one year.

\section{Epidural Injections:}

- In the diagnostic or stabilization phase, a patient may receive injections at intervals of no sooner than one week and preferably two weeks except for blockade in cancer pain or when a continuous administration of local anesthetic is employed for RSD.

- In the treatment or therapeutic phase (after the stabilization is completed), the frequency of interventional techniques should be two months or longer between each injection provided that at least $>50 \%$ relief is obtained for six weeks. However, if the neural blockade is applied for different regions, they can be performed at intervals of no sooner than one week and preferably two weeks for most type of blocks. The therapeutic frequency must remain two months for each region.

- In the diagnostic or stabilization phase, the number of injections should be limited to no more than four times except for RSD, in which case six times should be reasonable.

- In the treatment or therapeutic phase, the interventional procedures should be repeated only as necessary judging by the medical necessity criteria and these should be limited to a maximum of six times.
Under unusual circumstances with a recurrent injury, carcinoma, or reflex sympathetic dystrophy, blocks may be repeated at intervals of 6 weeks after stabilization in the treatment phase.

\section{Percutaneous Lysis of Adhesions:}

- For percutaneous non-endoscopic adhesiolysis with a 3-day protocol, 2 to 3 interventions per year are recommended; with a 1-day protocol, a maximum of four times per year is recommended.

- For endoscopic adhesiolysis, it is recommended that there be no more than two interventional procedures per year.

\section{Sympathetic Blocks:}

- In the diagnostic or stabilization phase, a patient may receive injections at intervals of no sooner than one week and preferably two weeks except in cancer pain or when a continuous administration of local anesthetic for sympathetic block is employed. However, the total number of injections in the stabilization phase should be limited to 4 to 6 .

- In the treatment or therapeutic phase, that is after the stabilization phase, the frequency of sympathetic blocks should be limited to two months or longer between each injection provided that at least greater than $50 \%$ relief is obtained for six weeks.

\section{Sacroiliac Joint Injections:}

- In the diagnostic or stabilization phase, a patient may receive injections at intervals of no sooner than one week and preferably two weeks.

- In the treatment or therapeutic phase (after the stabilization is completed), the frequency should be two months or longer between each injection provided that at least $>50 \%$ relief is obtained for six weeks. However, if the neural blockade is applied for different regions, they can be performed at intervals of no sooner than one week and preferably two weeks for most type of blocks. The therapeutic frequency must remain at two months for each region.

In the diagnostic or stabilization phase, the number of injections should be limited to no more than four times.

- In the treatment or therapeutic phase, sacroiliac 
joint injections should be repeated only as necessary judging by the medical necessity criteria and these should be limited to a maximum of six times for local anesthetic and steroid blocks for a period of one year.

\section{Trigger Point Injections:}

- In the diagnostic or stabilization phase, a patient may receive trigger point injections at intervals of no sooner than one week and preferably two weeks.

- In the treatment or therapeutic phase (after the stabilization is completed), the frequency should be two months or longer between each injection provided that at least $>50 \%$ relief is obtained for six weeks.

- In the diagnostic or stabilization phase, the number of trigger injections should be limited to no more than four times per year.

- In the treatment or therapeutic phase, the trigger point injections should be repeated only as necessary judging by the medical necessity criteria and these should be limited to a maximum of six times for local anesthetic and steroid injections.

Under unusual circumstances with a recurrent injury or cervicogenic headache trigger point injections may be repeated at intervals of six weeks after stabilization in the treatment phase.

Combination of Blocks/Interventions: It may be essential to combine, in certain circumstances, more than one block. This may include an epidural for the cervical region and facet-joint blocks for the lumbar region; epidural and facet-joint blocks for the same region in case of identification of pain generators from both sources; a sympathetic block and facet-joint block if there are two different sources of pain or if two different regions are affected in combination with trigger-point injections. Consequently, blocks also may be combined with other interventional techniques.

\section{OUTCOMES AND COST-EFFECTIVENESS}

Outcomes may be assessed by evaluation of the quality of life, which is also known as functional status, health status, health-related quality of life; well-being of the patient, satisfaction with care, health services utilization/economic analysis, and medical findings (793-802). The quality-oflife assessment is designed to evaluate the patient's abilities to function in his/her own world. Physical functioning measures the ability to perform physical activities such as walking, climbing stairs, or carrying things. Evaluation focuses on the patient's major perceived functional impairments, improvement in areas such as playing with children/grandchildren, having sexual relations, returning to work, going to school, homemaking or performing other activities of daily living. Quality of life also measures social functioning, which determines whether health problems affect normal social activities, such as seeing friends or participating in group activities.

Similarly, confusion abounds over what is meant by the term cost-effectiveness. Cost-effectiveness analysis has taken on an increasingly large role in health care policy debates about interventions for various types of interventions in managing low back pain. Growing health care costs and productivity losses, disappointing treatment results, and changing beliefs about health and pain have led to this increasing concern about the amount of money spent on chronic pain in general and low back pain in particular. In recent years, more and more studies in the field of the management of chronic low back pain have been incorporating cost issues in their analysis (795, 803-809). While economic evaluation designs describe cost minimizationanalysis (CMA), cost-benefit analysis (CBA), cost-effectiveness analysis (CEA), or cost-utility analysis (CUA), in chronic low back pain, CEA and CUA would be the most appropriate methods to use, since in these studies the effects are measured in natural units and quality of life. The outcome measures used in CEA studies in chronic pain research mainly include outcomes, such as disability days saved, pain-free days, or improved quality of life, etc. (803). Cost of inpatient chronic pain programs range from $\$ 17,000$ to $\$ 25,000$ and the cost of outpatient treatment programs range from $\$ 7,000$ to $\$ 10,000$ (802). In addition, chronic pain patients may incur health care bills in excess of $\$ 20,000$ annually for repetitive and, in some cases, redundant diagnostic work ups, physical therapy, psychological interventions, and drugs. Guo and colleagues (810) estimated that back pain accounted for 150 million lost work days in the United States every year, which worked out to be about $\$ 14$ billion in wage costs alone. The study showed that the magnitude of the back pain problem is so large that even a $1 \%$ reduction in overall prevalence could considerably reduce morbidity and save billions of dollars. The cost-effectiveness of lumbar discectomy for the treatment of herniated intervertebral discs has been based on the conclusion that surgery increased the average qualityadjusted life expectancy by 0.43 years during the decade following treatment compared to conservative treatment, a result comparable to extending a healthy life by five 
months (807). Malter et al (807) also concluded that for carefully selected patients with herniated discs, surgical discectomy is a cost-effective treatment at a discounted cost of $\$ 12,000$ per discectomy or $\$ 29,000$ per life year adjusted for quality. However, this study did not take into consideration chronic pain patients when initial surgical treatment for herniated disc fails. In such a study, it was shown that the success of a second operation was 50\%, with an additional $20 \%$ considering themselves worse after the surgery (266). With a third procedure, the success rate was $30 \%$, with $25 \%$ considering themselves worse; and after four operations, only a $20 \%$ success rate was achieved, with $45 \%$ of these patients considering themselves worse (266). Hence, if additional costs of repeat surgery are taken into consideration, the cost of lumbar surgery will probably be much higher. Kuntz et al (809) studied the cost-effectiveness of fusion with and without instrumentation for patients with degenerative spondylolisthesis and spinal stenosis. They showed that laminectomy with a non-instrumental fusion costs $\$ 56,500$ per qualityadjusted year of life versus laminectomy without fusion. The cost-effectiveness ratio of instrumented fusion com- pared with noninstrumented fusion was $\$ 3,112,800$ per quality-adjusted year of life (809). However, they also stated that if the proportion of patients experiencing symptom relief after instrumented fusion was $90 \%$ as compared with $80 \%$ for patients with non-instrumented fusion would $\$ 82,400$ per quality-adjust year of life. Mueller-Schwefe and colleagues (808), in evaluating the cost-effectiveness of intrathecal therapy for pain secondary to failed back surgery syndrome, compared alternative therapies for achieving a defined outcome, reporting the cost of medical management to be $\$ 17,037$ per year, or $\$ 1,420$ per month. They also showed that intrathecal morphine delivery resulted in lower cumulative 60 -month costs of $\$ 16,579$ per year and $\$ 1,382$ per month.

The cost-effectiveness evaluations for blind interlaminar, fluoroscopically directed caudal or transforaminal epidural injections for the management of low back pain showed the cost-effectiveness of caudal epidural steroids to be $\$ 3,635$ and transforaminal steroids to be $\$ 2,927$ per year, in stark contrast to blind interlaminar lumbar epidural steroid injections at $\$ 6,024$ per year (553). Cost-effective-

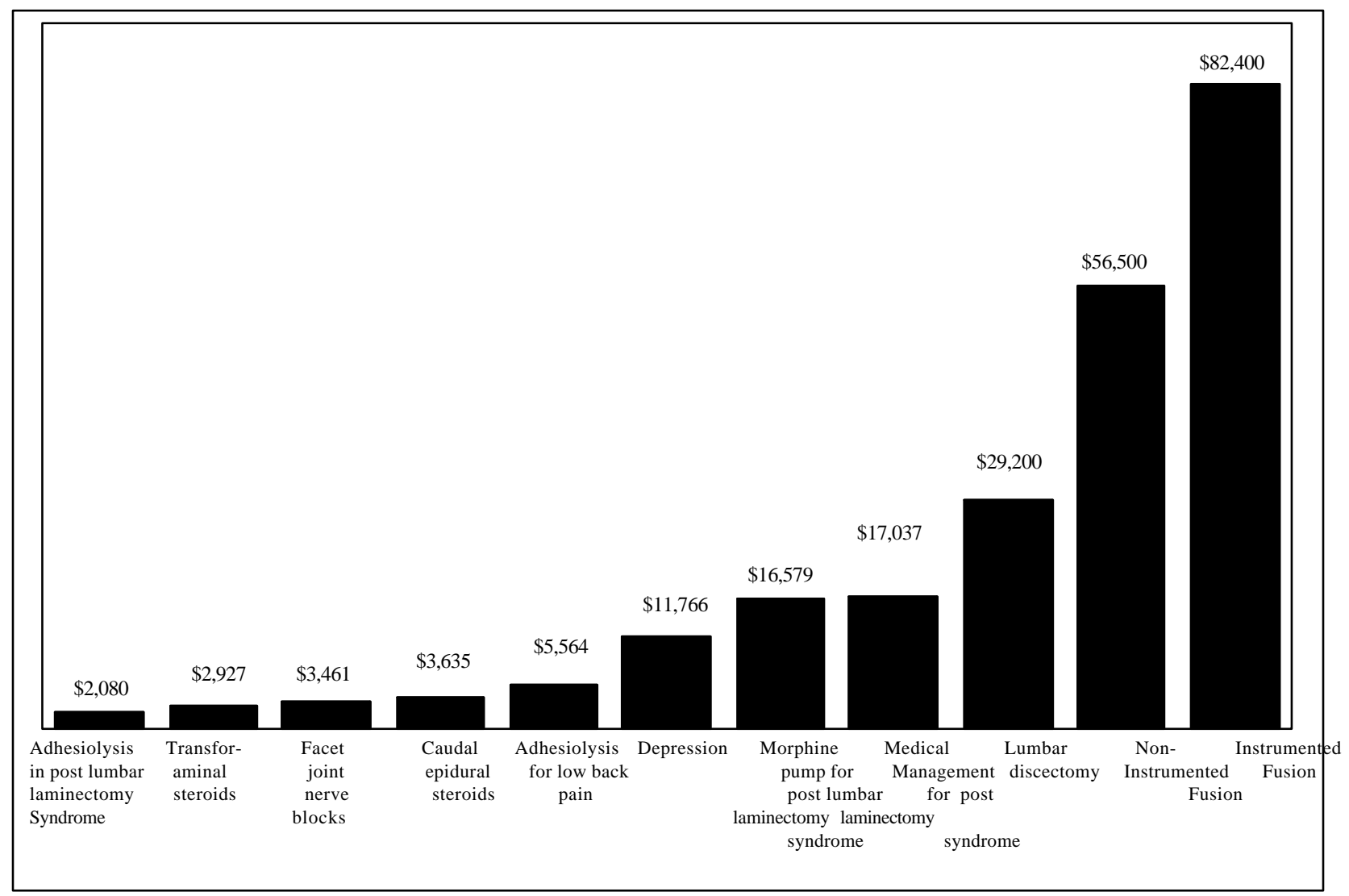

Fig. 5. Cost effectiveness of various types of therapy in managing medical conditions including chronic low back pain 
ness of percutaneous nonendoscopic adhesiolysis and hypertonic saline neurolysis was demonstrated to be $\$ 5,564$, for improvement of one year of quality of life for patients with chronic low back pain nonresponsive to numerous other modalities of treatment (647). Similarly, the costeffectiveness with nonendoscopic adhesiolysis was shown to be $\$ 2,028$ per year, whereas it was $\$ 7,020$ with endoscopic adhesiolysis in postlumbar laminectomy patients (649).

Evaluation of cost-effectiveness of lumbar facet joint nerve blocks, with or without steroids, by Manchikanti et al (481) showed that one year improvement of quality of life was achieved at $\$ 3,461$. This is similar to various investigations in the past with neural blockade but also significantly better than the cost-effectiveness, either with intrathecal morphine delivery, lumbar laminectomy, or lumbar laminectomy, with or without instrumented fusion. In addi- tion, the interpretation of the current results should be placed in the context of other surgical interventions and other modalities of treatments also. Lave et al (811) demonstrated the cost-effectiveness of medical treatment of depression management as $\$ 11,766$ per year of quality adjusted life. It was also shown that a simple reduction of diastolic pressure from 110 to $90 \mathrm{~mm}$ of hg was achieved at a cost of $\$ 16,330$ for a 60-year old man in 1974 (793). Total hip arthroplasty for osteoarthritis of the hip costs $\$ 61,000$ per quality adjusted year of life gained (812). Lumbar diskectomy for the treatment of herniated intervertebral discs cost $\$ 39,500$ per quality adjusted year of life gained $(807,809)$; coronary artery bypass grafting for patients with triple-vessel coronary disease and severe left ventricular function cost $\$ 41,800$ per year quality-adjusted year of life gained (813). Hence, it appears that precision percutaneous injection therapy and other interventional techniques are cost effective if performed properly, as

Table 16. Effectiveness of various commonly used interventional techniques in managing chronic pain

\begin{tabular}{|c|c|c|c|c|c|}
\hline Intervention & $\begin{array}{l}\text { Randomized } \\
\text { Trials }\end{array}$ & $\begin{array}{l}\text { Observational } \\
\text { Studies }\end{array}$ & $\begin{array}{l}\text { Effectiveness } \\
\text { /Evidence } \\
\text { Strength }\end{array}$ & $\begin{array}{l}\text { Complications } \\
\text { /Risks }\end{array}$ & $\begin{array}{l}\text { Cost per } \\
\text { One Year of } \\
\text { Qualityof Life }\end{array}$ \\
\hline $\begin{array}{l}\text { Intra-articular facet joint } \\
\text { injections }\end{array}$ & Six & Multiple & Moderate/Limited & Minimal & NA \\
\hline Facet joint nerve blocks & Four & Two & Moderate & Minimal & $\$ 3461$ \\
\hline $\begin{array}{l}\text { Medical branch } \\
\text { neurotomy }\end{array}$ & Four & Multiple & Strong & Minimal & NA \\
\hline Caudal epidural steroids & Six & Multiple & Strong & Minimal & $\$ 3635$ \\
\hline $\begin{array}{l}\text { Interlaminar epidural } \\
\text { steroids }\end{array}$ & Thirteen & Multiple & Moderate/Limited & Minimal & $\$ 6024$ \\
\hline $\begin{array}{l}\text { Transforaminal epidural } \\
\text { steroids }\end{array}$ & Five & Multiple & Strong/Moderate & Minimal & $\$ 2927$ \\
\hline $\begin{array}{l}\text { Epidural lysis of } \\
\text { adhesions }\end{array}$ & One & Three & Moderate & Minimal & $\begin{array}{l}\$ 2080 \mathrm{TO} \\
\$ 5564\end{array}$ \\
\hline Spinal endoscopy & None & Three & Limited & Minimal & $\begin{array}{l}\$ 7020 \mathrm{TO} \\
\$ 8127\end{array}$ \\
\hline $\begin{array}{l}\text { Intradiscal electrothermal } \\
\text { annuloplasty }\end{array}$ & One & Multiple & Moderate & Minimal & NA \\
\hline Spinal cord stimulation & Three & Multiple & Moderate & Significant & NA \\
\hline Intrathecal pumps & None & Multiple & Moderate & Minimal & $\$ 16,579$ \\
\hline Sympathetic blocks & None & Multiple & Limited & Minimal & NA \\
\hline Trigger point injections & Seven & Multiple & Moderate/Limited & Minimal & NA \\
\hline
\end{tabular}


shown in Fig. 5.

Thus, determining whether a service is worthwhile involves a number of different issues. It involves not only knowing whether the various components of the intervention are effective, but also how much they cost and if the delivery system is efficient. The preceding discussion concentrated on trying to determine whether interventional techniques in managing chronic pain could be shown to be effective through a systematic review. To achieve this goal, numerous relevant studies and reviews were reviewed for the quality and application to the subject of interventional techniques in chronic pain. Finally, the relative efficiency and safety of the possible interventions, and then the cost, have to be the key determinants. Table 16 shows various interventional techniques in managing chronic pain classified by evidence of effectiveness, as well as risk of side effects and cost of the procedure.

\section{CONCLUSION}

The practice guidelines for interventional techniques in the management of chronic pain were developed utilizing the best available evidence combined with consensus. These guidelines include discussions of the purpose, rationale, and importance. The guidelines also have discussed the importance of randomized controlled trials, the development of type and strength of efficacy evidence and various controversial aspects relating to guidelines. Chronic pain and its epidemiology, as well as discussion of chronic pain vs chronic pain syndrome, the pathophysiologic basis of persistent pain, and the evaluation of the patient presenting with chronic pain, have been discussed. Diagnostic and therapeutic interventional techniques are discussed extensively including all types of evidence available from randomized clinical trials as well as some observational studies. The levels of effectiveness for the most commonly used interventions were developed based on review of diagnostic and therapeutic interventional techniques. Additionally, effectiveness evidence and an algorithmic approach to managing a patient presenting with chronic spinal pain were also developed.

\section{ACKNOWLEDGMENTS}

We would like to thank Bert Fellows, Denise Pratt, Tonie Hatton, Vidyasagar Pampati, and many of the members of the organization who have assisted in the preparation of this manuscript.

\section{REFERENCES}

1. Committee to Advise the Public Health Service on Clinical Practice Guidelines, Institute of Medicine. Field MJ, Lohr KN (eds). Clinical Practice Guidelines. Directions of a New Program. National Academy Press; Washington, 1990.

2. Harris JS. Development, use, and evaluation of clinical practice guidelines. JOEM 1997; 39:23-34.

3. McQuay H, Moore A. An evidence-based resource for pain relief. Oxford University Press. Oxford, New York, 1998.

4. Woolf SH. Practice guidelines: A new reality in medicine. I. Recent developments. Arch Intern Med 1990; 150:1811-1818.

5. Woolf SH. Practice guidelines, a new reality. Medicine II. Methods of developing guidelines. Arch Intern Med 1992; 152:946-952.

6. Turk MA. Practice guidelines. In Gonzalez EG, Materson RS (eds). Thenonsurgical management of acute low back pain. Demos Vermande, New York, 1997, pp 5-14.

7. McQuay H, Moore A. Introduction: Anevidencebased resource for pain relief. Oxford University Press, Oxford, New York, 1998, pp 1-4.

8. Bombardier C, Esmail R, Nachemson AL et al. The Cochrane collaboration back review group for spinal disorders. Spine 1997; 22:837-840.

9. Leape LL. Practice guidelines and standards: An overview. QRB Qual Rev Bull1990; 16:42-49.

10. Sackett D, Richardson WS, Roseberg W et al. Evidence based medicine. Churchill Livingstone, Philadelphia, 1996.

11. Tinker JH, Dull DL, Caplan RA. Role of monitoring devices in the prevention of anesthetic mishaps: A closed claim analysis. Anesthesiology 1989; 71:541546.

12. Shoemaker TS. Practice policies in anesthesia: A foretaste of practice in the twenty-first century. Anesth Analg 1995; 388-403.

13. Shoemaker TS. Practice guidelines for pain management. In Ashburn MA and Rice LJ (eds), The Management of Pain. Churchill Livingstone. Philadelphia, 1998, pp 205-226.

14. Pierce EC. The development of anesthesia guidelines and standards. QRB Qual Rev Bull 1990;16:61-64.

15. McPherson K, Strong PM, Epstein A et al. Regional variations in the use of common surgical procedures: 
Within and between England and Wales, Canada and the United States of America. Soc Sci Med 1981; 15A:273-288.

16. Pilote L, Califf RM, Sapp S et al. Special articles: Regional variation across the United States in the management of acute myocardial infarction. NEngl JMed 1995; 333:565-572.

17. Roos NP, Roos KK. Surgical rate variations. Do they reflect the health or socioeconomic characteristics of the population? Med Care 1982; 20:945-958.

18. Eisenberg JM. Clinical economics: A guide to the economic analysis of clinical practices. JAMA 1989; 262:2879-2886.

19. Leape LL. Park RE, Solomon DH et al. Does inappropriate use explain small-area variations in the use of health care services? JAMA 1990;263:669-672.

20. Eddy DM. Comparing benefits and harms: The balance shifts. JAMA 1990; 263:2493-2501.

21. Manchikanti L. Dr. No. Managed care: Clinical guidelines. Pain Physician 2000;3:3-6.

22. Carter A. Clinical practice guidelines. Can Med Assoc $J$ 1992; 147:1649-1650.

23. Battista RN, Hodge MJ. Clinical practice guidelines: Between science and art. Can Med Assoc J1993; 148:385.

24. Brook Robert H. Practice guidelines and practicing medicine. Are they compatible? JAMA 1989; 262:3027-3030.

25. Marwick C. Will evidence-based practice help span the gulf between medicine and law? JAMA 2000; 283:2775-2778.

26. Cabana MD, Rand CS, Powe NR et al. Why don't physicians follow clinical practice guidelines? A framework for improvement. JAMA 1999; 282:14581465.

27. Shaneyfelt TM, Mayo-Smith MF, Rothwangl J. Are guidelines following guidelines? The methodological quality of clinical practice guidelines in the peer-reviewed medical literature. JAMA 1999; 281:19001905.

28. Bigos SJ, Boyer OR, Braen GR et al. Acute Low Back Problems in Adults . Clinical Practice Guideline Number 4. AHCPR Publication No. 95-0642. Agency for Health Care Policy and Research, Public Health Service, US Department of Health and Human Services, Rockville, December 1994.

29. Spitzer WO, Leblanc FE, Dupuis M (eds). Quebec Task Force on Spinal Disorders. Scientific approach to the assessment and management of activity-related spinal disorders: A monograph for clinicians. Spine 1987; (suppl) 12:1-59.

30. Spitzer WO, Skovron ML, Salmi LR et al. Scientific monograph of the Quebec Task Force on whiplashassociated disorders: Redefining "whiplash" and its management. Spine 1995; (Suppl) 20:1-73.

31. Practice guidelines for chronic pain management. A report by the American Society of Anesthesiologists Task Force on Pain Management, Chronic Pain Section. Anesthesiology 1997; 86:995-1004.

32. Bogduk N. International Spinal Injection Society guidelines for the performance of spinal injection procedures. Part 1: Zygapophyseal joint blocks. Clin J Pain 1997; 13:285-302.

33. Manchikanti L, Singh V, Bakhit CE et al. Interventional techniques in the management of chronic pain: Part 1.0. Pain Physician 2000; 3:7-42.

34. Campbell JK, Penzien DB, Wall EM. Evidence-based guidelines for migraine headache: Behavorial and physical treatments. 2000. Available at:

http://www.aan.com/public/practiceguidelines/ headache_gl.htm.

35. American Pain Society. Guideline for the management of acute and chronic pain in sickle-cell disease. American Pain Society, Glenview, 1999.

36. American Geriatrics Society. The management of chronic pain in older persons: New guidelines from the American Geriatrics Society. J Am Geriatr Soc 1998; 46:128-150.

37. Sanders SH, Rucker KS, Anderson KO et al. Clinical practice guidelines for chronic non-malignant pain syndrome patients. J Back Musc Rehabil 1995;5:115120.

38. Sanders SH, Harden RN, Benson SE et al. Clinical practice guidelines for chronic non-malignant pain syndrome patients II: An evidence-based approach.J Back Musc Rehabil 1999; 13:47-58.

39. Dickersin K, Manheimer E. The Cochrane collaboration: Evaluation of health care and services using systematic reviews of the results of randomized controlled trials. Clin Obstet Gynecol 1998; 41(2):315-331.

40. Tulder MWV, Assendelft JJ, Koes BW et al. Method guidelines for systematic reviews in the Cochrane collaboration back review group for spinal disorders. Spine 1997; 22:2323-2330.

41. Manchikanti L. The role of neural blockade in the management of chronic low back pain. Pain Digest 1999; 9:166-181.

42. Manchikanti L. Neural blockade in cervical pain syndromes. Pain Physician, 1999;2:65-84.

43. Nash TP. Current guidelines in the use of epidural steroids in the United Kingdom. Pain Digest 1999; 9:231-232.

44. Abram SE. Current guidelines in the use of epidural steroids in the United States of America. Pain Digest 1999; 9:233-234.

45. Koes BW, Scholten RJPM, Mens JMA et al. Efficacy of epidural steroid injections for low back pain and sciatica: A systematic review of randomized clinical trials. Pain 1995; 63:279-288.

46. Koes BW, Scholten R, Mens JMA et al. Epidural steroid injections for low back pain and sciatica. An updated systematic review of randomized clinical trials. 
Pain Digest 1999; 9:241-247.

47. Bogduk N, Christophidis N, Cherry D et al. Epidural use of steroids in the management of back pain. Report of working party on epidural use of steroids in the management of back pain. National Health and Medical Research Council. Canberra, Commonwealth of Australia, 1994, pp 1-76.

48. Watts RW, Silagy CA. A meta-analysis on the efficacy of epidural corticosteroids in the treatment of sciatica. Anaesth Intens Care 1995; 23:564-569.

49. Mugford HK. Conflicting conclusions from two systematic reviews of epidural steroid injections for sciatica: Which evidence should general practitioners heed? Br J Gen Pract 1999; 49:57-61.

50. Jacobson L, Mariano A, Chabal C et al. Beyond the needle. Expanding the role of anesthesiologists in the management of chronic non-malignant pain. Anesthesiology 1997; 87:1210-1218.

51. Kepes ER, Duncalf D. Treatment of backache with spinal injections of local anesthetics, spinal and systemic steroids. Pain 1985; 22:33-47.

52. Benzon HT. Epidural steroid injections for low back pain and lumbosacral radiculography. Pain 1986; 24:277.

53. Spaccarelli KC. Lumbar and caudal epidural steroid injections. Mayo Clin Proc 1996; 71:169.

54. Abram SE. Treatment of lumbosacral radiculopathy with epidural steroids. Anesthesiology 1999;91:19371941.

55. Rowlingson J. Epidural steroids in treating failed back surgery syndrome. Anesth Analg 1999; 88:240-242.

56. Manchikanti L. Facet joint pain and the role of neural blockade in its management. Cur Rev Pain 1999; 3:348-358.

57. Bogduk N. Epidural steroids for low back pain and sciatica. Pain Digest 1999; 9:226-227.

58. Manchikanti L. Transforaminal lumbar epidural steroid injections. Pain Physician 2000; 3:374-398.

59. Waldman SD, Waldman KA, Siwek SM. Interventional pain management, programming for success: In Waldman SD (ed), Interventional pain management. Second Edition. W.B. Saunders Company, Philadelphia 2000, pp 79-82.

60. Benzon HT, Molly RE. Outcomes, efficacy, and compliances from management of low back pain. In Raj PP, Abrams BM, Benzon HT et al (eds), Practical management of pain. Third Edition, Mosby, Philadelphia 2000, pp 891-903.

61. Godlee F. Clinical evidence. BMJ Publishing Group, London, June 2000; pp 486-572.

62. Gonzalez EG, Materson(eds). Thenonsurgicalmanagement of acute low back pain. Demos Vermane, New York 1997.

63. Rainville J, Carlson N, Polatin P. Exploration of physicians' recommendations for activities in chronic low back pain. Spine 2000; 17:2210-2220.
64. Nunn CM. Pathways, guidelines, and cookbook medicine: Are we all becoming Betty Crocker? JCOM 1997; 4:17-24.

65. Attributes to guide the development of practice parameters. American Medical Association, Chicago, 1994.

66. Koes BW, Bouter LM, Van Der Heijden GJMGV. Methodological quality of randomized clinical trials on treatment efficacy in low back pain. Spine 1995; 20:228-235.

67. Tulder MWV, Koes BW, Bouter LM. Conservative treatment of acute and chronic nonspecific low back pain. A systematic review of randomized controlled trials of the most common interventions. Spine 1997; 22:2128-2156.

68. Fishbain D, Cutler RB, Rosomoff HL et al. What is the quality of the implemented meta-analytic procedures in chronic pain treatment meta-analyses? ClinJ Pain 2000; 16:73-85.

69. Turner JA, Loeser JD, Bell KG. Spinal cord stimulation for chronic low back pain. A systematic literature synthesis. Neurosurgery 1995; 37:1088-1098.

70. Weinstein JN. The tortoise and the hare. Is there a place in spine surgery for randomized trials? Spine 1999; 23:2548-2549.

71. Winter RB. The prospective, randomized, controlled clinical trial in spine surgery. Fact or fiction? Spine 1999; 23:2550-2552.

72. Carey TS. Randomized controlled trials in surgery. An essential component of scientific progress. Spine 1999; 23:2553-2555.

73. Fairbank J. Randomized controlled trials in the surgical management of spinal problems. Spine 1999; 23:2556-2563.

74. Tosteson TD. Point of view. Spine 1999;24:25622563.

75. Turk DC, Okifuji A. Treatment of chronic pain patients. Clinical outcomes, cost-effectiveness, and costbenefits of multidisciplinary pain centers. Crit Rev Phys Rehabil Med 1998; 10:181-208.

76. Concato J, Shah N, Horwitz RI. Randomized, controlled trials, observational studies, and the hierarchy of research designs. NEngl J Med 2000; 342:18871892.

77. Pocock S, Elbourne D. Randomized trials or observational tribulations? (editorial) N Engl J Med2000; 342:1907-1909.

78. Hopwood MB, Manning DC. Lumbar epidural steroid injections. Reg Anesth Pain Med 1999;24:5-7.

79. Turk DC. Statistical significance and clinical significance are not synonyms! Clin J Pain 2000; 16:186187.

80. Schulz KF, Chalmers I, Hayes RJ et al. Empirical evidence of bias: Dimensions of methodological quality associated with estimates of treatment effects in controlled trials. JAMA 1995; 273:408-412.

81. Jadad AR, Carroll D, Moore A et al. Developing a database of published reports of randomized clinical 
trials in pain research. Pain 1996; 66:239-246.

82. McQuay H, Moore A. Finding all the relevant trials. An evidence based resource for pain relief. Oxford University Press, Oxford, New York, 1998, pp 5-9.

83. Sanders SH. Integrating practice guidelines for chronic pain: From the Tower of Babel to the Rosetta Stone. APS Bulletin 2000; 10(6):1-7.

84. de Jong RH. BACKLASH: AHCPR practice guideline for acute low back pain. Pain Digest 1996; 6:1-2.

85. Schweitzer A. On the Edge of the Primeval Forest. Macmillan, New York, 1931; 62.

86. Gureje O, Von Korff M, Simon GE et al. Persistent pain and well being: A World Health Organization Study in Primary Care. JAMA 1998; 280:147-151.

87. Cousins MJ. Pain: The past, present, and future of anesthesiology? The E.A. Rovenstine Memorial Lecture. Anesthesiology 1999; 91:538-551.

88. Hill CS. When will adequate pain treatment be the norm? JAMA 1995; 274:1881-1882.

89. Cleeland CD, Gonin R, Hatfield AK et al. Pain and its treatments in outpatients with metastatic cancer. NEngl J Med 1994; 330:592-596.

90. Foley KM. The treatment of cancer pain. NEngl J Med 1985; 313:84-95.

91. Dahl JL. Improving the practice of pain management. JAMA 2000; 284:2785.

92. Marks RM, Sachar EJ. Undertreatment of medical patients with narcotic analgesics. Ann Intern Med 1973; 78:173-181.

93. Donovan M, Dillon P, McGuire L. Incidence and characteristics of pain in a sample of medical-surgical inpatients. Pain 1987; 30:69-87.

94. War SE, Gordon D. Application of the American Pain Society quality assurance standards. Pain 1994; 56:299-306.

95. Miaskowski C, Nichols R, Brody R et al. Assessment of patient satisfaction utilizing the American Pain Society's quality assurance standards on acute and cancer-related pain. J Pain Sym Manage 1994; 9:5-11.

96. Fanuele JC, Birkmeyer NJ, Abdu WA et al. The impact of spinal problems on the health status of patients. Have we underestimated the effect? Spine 2000; 25:1509-1514.

97. Perquin CW, Hazebroek-Kampschreur AA, Hunfeld JA et al. Chronic pain among children and adolescents: Physician consultation and medication use. Clin J Pain 2000; 16:229-235.

98. Menefee LA, Cohen MJ, Anderson WR et al. Sleep disturbance and nonmalignant chronic pain: A comprehensive review of the literature. Pain Med 2000; 1:156-172.

99. Hoffmann DE. Pain management and palliative care in the era of managed care: Issue for health insurers. $J$ Law, Med \& Ethics 1998;26:267-289.

100. Helme RD, Gibson SJ. Pain in older people. In Crombie IK, Croft PR, Linton SJ et al (eds). Epidemiology of pain. IASP Press; Seattle, 1999, pp 103-112.
101. Bressler HB, Keyes WJ, Rochon PA et al. The prevalence of low back pain in the elderly. A systemic review of the literature. Spine 1999; 24:1813-1819.

102. Pahor M, Guralnik JM, Wan JY. Lower body osteoarticular pain and dose of analgesic medications in older disabled women: The Women's Health and Aging Study. Am J Public Health 1999; 89:930-934.

103. Manchikanti L. Epidemiology of low back pain. Pain Physician 2000; 3:167-192.

104. Manchikanti L. Chronic low back pain in the elderly: Part 1. AJPM 1997; 7:104-117.

105. Manchikanti L. Chronic low back pain in the elderly: Part 2. AJPM 1997; 7:133-145.

106. Asch SM, Sloss EM, Hogan C et al. Measuring underuse of necessary care among elderly Medicare beneficiaries using inpatient and outpatient claims. JAMA 2000; 284:2325-2333.

107. Bonica JJ. Definitions and taxonomy of pain. In Bonica JJ, Loessor JD, Chapman CR et al (eds). The Management of Pain, Second Edition. Lea \& Febiger, Philadelphia, 1990; Vol. 1: pp 18-27.

108. The quality indicator, June 2000, p10.

109. Andersson HI, Ejlertsson G, Leden I et al. Chronic pain in a geographically defined general population: Studies of differences in age, gender, social class, and pain localization. Clin J Pain 1993; 9:174-182.

110. Crombie IK, Croft PR, Linton SJ et al (eds). Epidemiology of pain: A report of task force on epidemiology of the International Association for the Study of Pain. IASP Press, Seattle 1999.

111. Ferrell BR, Ferrell BA (eds). Pain in the elderly. A report of the task force on pain in the elderly of the international association for the study of pain. IASP Press, Seattle, 1996.

112. Farrell MJ, Gibson SJ, Helme RD. Chronic nonmalignant pain in older people. In Ferrell BR, Ferrell BA, (eds). Pain in the elderly. IASAP Press, Seattle 1996, pp 81-89.

113. Lawrence RC, Helmick CG, Arnett FC. Estimates of the prevalence of arthritis and selected musculoskeletal disorders in the United States. Arthritis \& Rheumatism 1998; 41:778-799.

114. Bovim G, Schrader H, Sand T. Neck pain in the general population. Spine 1994; 19:1307-1309.

115. Day LJ, Bovill EG, Trafton PG et al. Orthopedics. In Way LW (ed). Current surgical diagnosis and treatment. Appleton Lange, Connecticut, pp 1011-1104.

116. Scheolkov AP. Evaluation, diagnosis, and initial treatment of cervical disc disease. Spine State of the Art Reviews 1991; 5:167-176.

117. Linton SJ, Hellsing AL, Hallden K. A population based study of spinal pain among 35-45-year old individuals. Spine 1998; 23:1457-1463.

118. Cassidy D, Carroll L, Cote P: The Saskatchewan Health and Back Pain Survey. The prevalence of low back pain and related disability in Saskatchewan Adults. Spine 1998; 23:1860-1867. 
119. Côté DC, Cassidy JD, Carroll L. The Saskatchewan Health and Back Pain Survey. The prevalence of neck pain and related disability in Saskatchewan adults. Spine 1998; 23:1689-1698.

120. Côté DC, Cassidy JD, Carroll L. The factors associated with neck pain and its related disability in the Saskatchewan population. Spine 2000; 25:1109-1117.

121. Shekelle PG, Markovich M, Louie R. An epidemiologic study of episodes of back pain care. Spine 1995; 20:1668-1673.

122. Anderson GBJ, Svensson HO. The intensity of work recovery in low back pain. Spine 1983; 8:880-887.

123. Van Den Hoogen HJM, Koes BW, Deville W et al. The prognosis of low back pain in general practice. Spine 1997; 22:1515-1521.

124. Croft PR, Papageorgiou AC, Thomas E et al. Shortterm physical risk factors for new episodes of low back pain. Prospective evidence from the South Manchester Back Pain Study. Spine 1999; 24:1556-1561.

125. Carey TS, Garrett JM, Jackman A et al. Recurrence and care seeking after acute back pain. Results of a long-term follow-up study. Medical Care 1999; 37:157-164.

126. Miedema HS, Chorus AMJ, Wevers CWJ et al. Chronicity of back problems during working life. Spine 1998; 23:2021-2029.

127. Thomas E, Silman AJ, Croft PR et al. Predicting who develops chronic low back pain in primary care. A prospective study. Brit Med J 1999;318:1662-1667.

128. Badley EM, Tennant A. Changing profile of joint disorders with age. Findings from a postal survey of the population of Calderdale, West Yorkshire, United Kingdom. Ann Rheum Dis 1992; 51:366-371.

129. Bergstrom G, Bjelle A, Sorensen LB et al. Prevalence of symptoms and signs of joint impairment at age 79 . Scand J Rehab Med 1985; 17:173-182.

130. Bergstrom G, Bjelle A, Sundh V et al. Joint disorders at age 70,75, and 79 years. A cross-sectional comparison. BrJ Rheumatol 1986; 23:333-341.

131. Isaacsson A, Hanson BS, Ranstam J et al. Social network, social support, and the prevalence of neck and low back pain after retirement. A population study of men born in 1914 in Malmö, Sweden. Scand J Soc Med 1995; 23:17-22.

132. Lavsky-Shulan M, Wallace RB, Kohout FJ et al. Prevalence and functional correlates of low back pain in the elderly. The Iowa 65+ Rural Health Study. J Am Geriatr Soc 1985; 33:23-28.

133. March LM, Skinner J, Schwatz J et al. Musculoskeletal disability in an elderly population living independently. A random survey in the northern Sydney area. Aust NZ J Med 1992; 22:731.

134. Mobily PR, Herr KA, Clark MK et al. An epidemiologic analysis of pain in the elderly. The Iowa $65+$ Rural Health Study. J Aging Health 1994; 6:139-154. 135. Sternbach RA. Survey of pain in the United States.
The Nuprin pain report. Clin J Pain 1986; 3:49-53. Woo J, Ho SC, Lau J et al. Musculoskeletal complaints and associated consequences in elderly Chinese ages 70 years and over. JRheumatol 1994; 21:1927-1931.

137. Van Korff M, Dworkin SF, Le Resche L et al. An epidemiologic comparison of pain complaints. Pain 1988; 32:173-183.

138. Ferrell BA, Ferrell BR, Osterwell D. Pain in the nursing home. J Am Geriatr Soc 1990; 38:409-414.

139. Pain. In Guides to the evaluation of permanent impairment. Fourth Edition American Medical Association, Chicago, 1993, pp 303-311.

140. Merskey H, Bogduk N. Classification of chronic pain: Descriptions of chronic pain syndromes and definitions of pain terms. Second Edition. IASP Press, Seattle, 1994.

141. Rucker KS. Chronic pain evaluation. Butterworth/ Heinemann, Boston 2001.

142. Coccharella L, Andersson GBJ (eds). Pain. Guides to the evaluation of permanent impairment . Fifth Edition American Medical Association. AMA press, Chicago, IL, 2000, pp 565-591.

143. Corran TM, Farrell MJ, Helme RD et al. The classification of patients with chronic pain: Age as a contributing factor. Clin J Pain 1997; 13:207-214.

144. Hendler NH, Bergson C, Morrison C. Overlooked physical diagnoses in chronic pain patients involved in litigation. Part 2. Psychosomatics 1996;37:509517.

145. Hendler NH, Kolodny AL. Using medication wisely in chronic pain. Patient Care 1992; May 15:125.

146. Leigh JP, Markowitz S, Fahs M et al. Occupational injury and illness in the United States. Estimates of costs, morbidity, and mortality. Arch Intern Med 1997; 157:1557-1568.

147. Bogduk N. The innervation of the lumbar spine. Spine 1983; 8:286-293.

148. Suseki K, Takahashi Y, Takahashi Ket al. Innervation of the lumbar facet joints. Spine 1997; 22:477-485.

149. Bogduk N, Wilson AS, Tynan W. The human lumbar dorsal rami. J Anat 1982; 134:383-397.

150. Bogduk N. The clinical anatomy of the cervical dorsal rami. Spine 1982; 7:35-45.

151. Kellgren JH. The anatomical source of back pain. Rheumatol Rehab 1977; 16:3-12.

152. Hirsch D, Inglemark B, Miller M. The anatomical basis for low back pain. Acta Orthop Scand 1963;33:1.

153. Kuslich SD, Ulstrom CL, Michael CJ. The tissue origin of low back pain and sciatica: A report of pain response to tissue stimulation during operation on the lumbar spine using local anesthesia. Orthop Clin North Am 1991;22:181-187.

154. Mooney V, Robertson J. The facet syndrome. Clin Orthop 1976; 115:149-156.

155. McCall IW, Park WM, O'Brien JP. Induced pain referral from posterior elements in normal subjects.Spine 
1979; 4:441-446.

156. Marks R. Distribution of pain provoked from lumbar facet joints and related structures during diagnostic spinal infiltration. Pain 1989; 39:37-40.

157. Fukui S, Ohseto K, Shiotani M et al. Distribution of referral pain from the lumbar zygapophyseal joints and dorsal rami. Clin J Pain 1997; 13:303-307.

158. Fukui S, Ohseto K, Shiotani M et al. Referred pain distribution of the cervical zygapophyseal joints and cervical dorsal rami. Pain 1996; 68:79-83.

159. Dwyer A, Aprill C, Bogduk N. Cervical zygapophyseal joint pain patterns: A study in normal volunteers. Spine 1990; 6:453-457.

160. Aprill C, Dwyer A, Bogduk N. The prevalence of cervical zygapophyseal joint pain patterns II: A clinical evaluation. Spine 1990; 6:458-461.

161. Bogduk N, Marsland A. The cervical zygapophyseal joints as a source of neck pain. Spine 1988; 13:610617.

162. Bogduk N, Aprill C. On the nature of neck pain, discography, and cervical zygapophyseal joint blocks. Pain 1993; 54:213-217.

163. Bogduk N. Musculoskeletal pain: Toward precision diagnosis. Progress in pain researchandmanagement. In Jensen TS, Turner JA, Wiesenfeld-Hallin Z (eds). Proceedings of the 8th World Congress on Pain.IASP Press, Seattle, 1997, pp 507-525.

164. Bogduk N, Simons DG. Neck pain: Joint pain or trigger points. In: $\mathrm{H}$ Vaeroy and J Merskey (eds). Progress in Fibromyalgia and Myofascial Pain. Elsevier, Amsterdam, 1993,pp 267-273.

165. Slipman CW, Plastaras CT, Palmitier RA et al. Symptom provocation of fluoroscopically guided cervical nerve root stimulation: Are dynatomal maps identical to dermatomal maps? Spine 1998; 23:2235-2242.

166. Fortin JD, Dwyer AP, West S et al. Sacroiliac joint: pain referral maps upon applying a new injection/arthrography technique. Part I: Asymptomatic volunteers. Spine 1994; 19:1475-1482.

167. Fortin JD, Aprill CN, Ponthieux B et al. Sacroiliac joints: Pain referral maps upon applying a new injection/arthrography technique. Part II: Clinical evaluation. Spine 1994; 19:1483-1489.

168. Dreyfuss P, Tibiletti C, Dreyer SJ. Thoracic zygapophyseal joint pain patterns: A study in normal volunteers. Spine 1994; 19:807-811.

169. Dreyfuss P, Michaelsen M, Fletcher D. Atlanto-occipital and lateral atlanto-axial joint pain patterns. Spine 1994; 19:1125-1131.

170. Bogduk N, Marsland A. On the concept of third occipital headache. JNeurol Neurosurg Psychiatry1986; 49:775-780.

171. Barnsley L, Lord S, Bogduk N. Whiplash injury. Pain 1994; 58:238-307.

172. Simons DG. Myofascial trigger points, a possible explanation. Pain 1988; 69:207-212.

173. Fortin JD. Precision diagnostic disc injections. Pain
Physician 2000; 3:271-288.

174. Schwarzer AC, Aprill CN, Derby R et al. The prevalence and clinical features of internal disc disruption in patients with chronic low back pain. Spine 1995; 20:1878-1883.

175. Schwarzer AC, Derby R, Aprill CN et al. The value of the provocation response in lumbar zygapophysial joint injections. Clin J Pain 1994; 10:309-313.

176. Schwarzer AC, Wang S, Laurent R et al. The role of the zygapophysial joint in chronic low back pain. Aust N Z J Med 1992; 22:185.

177. Schwarzer AC, Derby R, Aprill CN et al. Pain from the lumbar zygapophysial joints: A test of two models. J Spinal Disord 1994; 7:331-336.

178. Schwarzer AC, Aprill CN, Derby R et al. Clinical features of patients with pain stemming from the lumbar zygapophysial joints. Is the lumbar facet syndrome a clinical entity? Spine 1994; 19:1132-1137.

179. Schwarzer AC, Wang S, Bogduk N et al. Prevalence and clinical features of lumbar zygapophysial joint pain: A study in an Australian population with chronic low back pain. Am Rheum Dis 1995; 54:100-106.

180. Schwarzer AC, Aprill CN, Derby R et al. The relative contributions of the disc and zygapophyseal joint in chronic low back pain. Spine 1994; 19:801-806.

181. Manchikanti L, Pampati VS, Fellows B et al. Prevalence of lumbar facet joint pain in chronic low back pain. Pain Physician 1999; 2:59-64.

182. Manchikanti L, Pampati VS, Bakhit CE et al. The diagnostic validity and therapeutic value of lumbar facet joint nerve blocks with or without adjuvant agents. Cur Rev Pain 2000; 4:337-344.

183. Manchikanti L, Pampati V, Fellows B et al. The inability of the clinical picture to characterize pain from facet joints. Pain Physician 2000; 3:158-166.

184. Barnsley L, Lord SM, Wallis BJ et al. The prevalence of chronic cervical zygapophyseal joint pain after whiplash. Spine 1995; 20:20-26.

185. Lord SM, Barnsley L, Wallis BJ et al. Chronic cervical zygapophysial joint pain with whiplash: A placebocontrolled prevalence study. Spine 1996; 21:17371745 .

186. Simmons DQ, Travell JG. The myofascial genesis of pain. Postgrad Med 1952; 11:425-434.

187. Han SC, Harrison P. Myofascial Pain Syndrome and Trigger-Point Management. Reg Anesth 1997; 22(1):89-101.

188. Travell JG, Simons DG. Myofascial pain and dysfunction: The trigger point manual. Lippincott Williams \& Wilkins, Baltimore, 1999, Vol 2.

189. Simons DG. Muscular pain syndromes. In Friction JR, Awad EA (eds). Advances in Pain Research and Therapy. Lippincott-Raven, Philadelphia, 1990, Vol 17.

190. Macfarlane GJ. Fibromyalgia and chronic widespread pain. In epidemiology of pain. Crombie IK, Croft PR, Linton SJ et al (eds), IASP Press, Seattle, 1999, 
pp 113-124.

191. Schwarzer AC, Aprill CN, Bogduk M. The sacroiliac joint in chronic low back pain. Spine 1995; 20:31-37.

192. Maigne JY, Aivakiklis A, Pfefer F: Results of sacroiliac joint double block and value of sacroiliac pain provocation test in 54 patients with low back pain. Spine 1996; 21:1889-1892.

193. Harden RN. A clinical approach to complex regional pain syndrome. Clin J Pain (Suppl) 2000; 16:S26-32.

194. Stanton-Hicks M. Complex regional pain syndrome (Type I, RSD; Type II, Causalgia): Controversies. Clin $J$ Pain 2000; 16:S33-40.

195. Veldman P, Reynen HM, Arntz IE et al. Signs and symptoms of reflex sympathetic dystrophy: Prospective study of 829 patients. Lancet $1993 ; 342: 1012$ 1016.

196. Allen G, Galer B, Schwartz L. Epidemiology of complex regional pain syndrome: A retrospective chart review of 134 patients. Pain 1999; 80:539-544.

197. Andersson HI, Ejlertsson E, Leden I et al. Characteristics of subjects with chronic pain, in relation to local and widespread pain report. ScandJRheumatol1996; 25:146-154.

198. Borenstein D. Prevalence and treatment outcome of primary and secondary fibromyalgia in patients with spinal pain. Spine 1995; 20:796-800.

199. Macfarlane GJ, Hunt IM, McBeth J et al. Chronic widespread pain in the community: Influences on health-care seeking behavior. J Rheumatol 1999; 26:413-419.

200. Magni G, Caldieron C, Rigatti-Luchini S et al. Chronic musculoskeletal pain and depressive symptoms in the general population. An analysis of the $1^{\text {st }}$ National Health and Nutrition Examination Survey Data. Pain 1990; 43:299-307.

201. Wolfe F. The relation between tender points and fibromyalgia symptom variables: evidence that fibromyalgia is not a discrete disorder in the clinic. Ann Rheum Dis 1997; 56:268-271.

202. Rachlin ES. Trigger point management. In Gonzalez EG, Materson RS (eds). The nonsurgical management of acute low back pain. Demos Vermande, New York, 1997, pp 151-158.

203. Frost FA, Jessen B, Singgarrd-Andersen J. A control double-blind comparison of mepivacaine injection versus saline injection for myofascial pain. Lancet 1980; 8167-8168.

204. Garvey TA, Marks MR, Wiesel SW. A prospective, randomized, double-blind evaluation of trigger-point injection therapy for low-back pain. Department of Orthopedic Surgery, The George Washington University Medical Center, Washington, DC, and The Cleveland Clinic, Cleveland, Ohio, 1989, pp 962-964.

205. Collee G, Dijkmans B, Cats A et al. Iliac crest pain syndrome in low back pain. A double-blind, randomized study of local injection therapy.J Rheumatol 1991 ;
18:1060-1063.

206. Bourne IHJ. Treatment of chronic back pain. Comparing corticosteroid-lignocaine injections with lignocaine alone. Practitioner 1984; 228:333-338.

207. Hameroff SR, Crago BR, Blitt CD et al. Comparison of bupivacaine, etidocaine, and saline for trigger point therapy. Anesth Analg 1981;60:752-755.

208. Fine PG, Milano R, Hare BD. The effects of myofascial trigger point injections are naloxone reversible. Pain 1988; 32:15-20.

209. Yaeger B, Shootsky SA. Double-blind, controlled study of different myofascial trigger point injection techniques. Pain 1987; 31:5292.

210. Kalauokalani DAK, Loeser JD. Phantom limb pain. In Crombie IK, Croft PR, Linton SJ et al (eds). Epidemiology of pain. IASP Press, Seattle, 1999, pp 143153.

211. Jensen TS, Krebs B, Nielsen J et al. Immediate and long-term phantom limb pain in amputees: incidence, clinical characteristics and relationship to -pre-amputation limb pain. Pain 1985;21:267-278.

212. Scher AI, Stewart WF, Lipton RB. Migraine and headache: A meta-analytic approach. In Crombie IK, Croft PR, Linton SJ et al (eds), Epidemiology of pain. IASP Press, Seattle, 1999, pp 159-170.

213. Zakrzewska JM, Hamlyn PJ. Facial pain. In Crombie IK, Croft PR, Linton SJ et al (eds), Epidemiology of pain. IASP Press, Seattle, 1999, pp 171-202.

214. Portenoy RK. Cancer pain: Epidemiology and syndromes. Cancer 1989; 63:2298.

215. Rasmussen BK, Jensen R, Schroll M, et al. Epidemiology of headache in a general population - A prevalence study. J Clin Epidemiol 1991;44:1147-1157.

216. Kirkaldy-Willis WH, Wedge JH, Yong-Hing K et al. Pathology and pathogenesis of lumbar spondylosis and stenosis. Spine 1978;3:319-327.

217. Handel JA, Knap J, Poletti S. The structural degenerative cascade. The cervical spine. In White $\mathrm{AH}$, Schofferaian, JA (eds). Spine Care. Diagnosis and conservative treatment. Mosby, St. Louis, 1995, Vol. 1: pp 16-23.

218. Fujiwara A, Tamai K, Yamato M et al. The relationship between facet joint osteoarthritis and disc degeneration of the lumbar spine: An MRI study. EurJ Spine 1999; 8:396-401.

219. Fujiwara A, Tamai K, An HS. The relationship between disc degeneration, facet joint osteoarthritis, and stability of the degenerative lumbar spine. JSpinal Disord 2000; 13:444-450.

220. Thompson RE, Pearcy MJ, Downing KJW. Disc lesions and the mechanics of the intervertebral joint complex. Spine 2000; 25:3026-3035.

221. Fujiwara A, Lim T, An H et al. The effect of disc degeneration and facet joint osteoarthritis on the segmental flexibility of the lumbar spine. Spine 2000; 25:3036-3044. 
222. Mixter WJ, Barr JS. Rupture of the intervertebral disc with involvement of the spinal canal. NEng JMed 1934; 211:210-215.

223. Semmes RE, Murphey MF. The syndrome of unilateral rupture of the sixth cervical intervertebral disc with compression of the seventh cervical nerve root. A report of four cases with symptoms simulating coronary disease. JAMA 1943; 121:1209.

224. Crock HV. Isolated lumbar disc resorption as a cause of nerve root canal stenosis. Clin Orthop 1976; 115:109-115.

225. Crock HV. A reappraisal of intervertebral disc lesions. Med J Aust 1970; 1:983-989.

226. Sehgal N, Fortin JD. Internal disc disruption and low back pain. Pain Physician 2000;3:143-157.

227. McCarron RF, Wimpee MW, Hudkins PG et al. The inflammatory effects of nucleus pulposus: A possible element in the pathogenesis of low back pain. Spine 1987; 12:760-764.

228. Saal JS, Franson RC, Dobrow R et al. High levels of inflammatory phospholipase A2 activity in lumbar disc herniations. Spine 1990; 15:674-678.

229. Kayama S, Konno S, Olmarker Ket al. Incision of the annulus fibrosus induces nerve root morphologic, vascular, and functional changes. Spine 1996; 21:25392543.

230. Chaoyang C, Cavanaugh JM, Ozaktay C et al. Effects of phospholipase $\mathrm{A}_{2}$ on lumbar nerve root structure and function. Spine 1997; 22:1057-1064.

231. Bobechko WT, Hircsch C. Autoimmune response to nucleus pulposus in the rabbit. J Bone Joint Surg 1965; 47B:574-580.

232. Harrington JF, Messier AA, Bereiter D et al. Herniated lumbar disc material as a source of free glutamate available to affect pain signals through the dorsal root ganglion. Spine 2000; 25:929-936.

233. Elves MW, Bucknill T, Sullivan MF. In vitro inhibition of leucocyte migration in patients with intervertebral disc lesions. Orthop Clin North Am 1975; 6:5965 .

234. Gertzbein SD. Degenerative disk disease of the lumbar spine: Immunological implications. Clin Orthop 1977; 129:68-71.

235. Gertzbein SD, Tait JH, Devlin SR. The stimulation of lymphocytes by nucleus pulposus in patients with degenerative disk disease of the lumbar spine. Clin Orthop 1977; 123:149-154.

236. Gertzbein SD, Tile M, Gross A. Autoimmunity in degenerative disc disease of the lumbar spine. Orthop Clin North Am 1975; 6:67-73.

237. Olmarker K, Blomquist J, Stromberg J et al. Inflammatogenic properties of nucleus pulposus. Spine 1995; 20:665-669.

238. Cavanaugh JM, Ozaktay AC, Vaidyanathan S. Mechano- and chemosensitivity of lumbar dorsal roots and dorsal root ganglia: An in vitro study. Trans Orthop Res Soc 1994; 19:109.
239. Kawakami M, Weinstein JN, Tamaki, et al. The difference in nociceptive potential of the nucleus pulposus and the anulus fibrosus. In Weinstein JN, Gordon SL (eds). Low Back Pain: A Scientific and Clinical Overview. American Academy of Orthopaedic Surgeons, 1996, Rosemont, pp 209-213.

240. Kawakami M, Tamaki T, Weinstein JN et al. Pathomechanism of pain-related behavior produced by allografts of intervertebral disc in the rat. Spine 1996; 21:2101-2107.

241. Kang JD, Georgescu HI, McIntyre-Larkin Let al. Herniated lumbar intervertebral discs spontaneously produce matrix metalloproteinases, nitric oxide, interleukin-6, and prostaglandin $\mathrm{E}_{2}$. Spine 1996; 21:271-277.

242. Olmarker K, Rydevik B, Nordborg C. Autologous nucleus pulposus induces neurophysiologic and histologic changes in porcine cauda equina nerve roots. Spine 1993; 181:1425-1432.

243. Grönblad M, Virri J, Tolonen J et al. A controlled immunohistochemical study of inflammatory cells in disc herniation tissue. Spine 1994; 19:2744-2751.

244. Jaffray D, O’Brien JP. Isolated intervertebral disc resorption: A source of mechanical and inflammatory back pain? Spine 1986; 11:397-401.

245. Kawakami M, Tamaki T, Hashizume $\mathrm{H}$ et al. The role of phospholipase $\mathrm{A}_{2}$ and nitric oxide in pain-related behavior produced by an allograft of intervertebral disc material to the sciatic nerve of the rat. Spine 1997; 22:1074-1079.

246. Marshall LL, Trethewie ER, Curtain CC. Chemical radiculitis: A clinical, physiological and immunological study. Clin Orthop 1977;129:61-67.

247. Murphy RW. Nerve roots and spinal nerves in degenerative disk diseases. Clin Orthop 1977; 129:46-60.

248. Nygaard OP, Mellgren SI, Osterud B. The inflammatory properties of contained and noncontained lumbar disc herniation. Spine 1997; 22:2484-2488.

249. Yabuki S, Kikuchi S, Olmarker K et al. Acute effects of nucleus pulposus on blood flow and endoneurial fluid pressure in rat dorsal root ganglia. Spine 1998; 23:2517-2523.

250. Yabuki S, Igarashi T, Kikuchi S. Application of nucleus pulposus to the nerve root simultaneously reduces blood flow in dorsal root ganglion and corresponding hindpaw in the rat. Spine 2000; 25:1471-1476.

251. Buckwalter JA. Aging and degeneration of the human intervertebral disk. Spine 1995; 20:1307-1314.

252. Hirsch C, Schajowicz F, Galante J. Structural changes in the cervical spine. Acta Orth Scand1967;109:6872.

253. Lees F, Turner JWA. Natural history and prognosis of cervical spondylosis. Br Med J1963; 2:1607-1610.

254. Ono K, Ota H, Tada K et al. Cervical myelopathy secondary to multiple spondylotic protrusions: A clinicopathologic study. Spine 1977; 2:109-125.

255. Weber H. The natural history of disc herniations and 
the influence of intervention. Spine 1994; 19:22342238.

256. Spangfort EV. The lumbar disc herniation. A computer aided analysis of 2504 operations. Acta Orthop Scand (Suppl) 1972; 142:1-95.

257. Michelsen JJ, Mixter WJ. Pain and disability of shoulder and arm due to herniation of the nucleus pulposus of cervical intervertebral discs. NEngl JMed 1944; 231:279.

258. Carette S, Lecaire R, Marcoux S et al. Epidural corticosteroid injections for sciatica due to herniated nucleus pulposus. N Engl J Med 1997; 336:1634-1640.

259. Ohnmeiss D, Vanharanta H, Ekholm J. Degree of disc disruption and lower extremity pain. Spine 1997; 22:1600-1605.

260. Frymoyer JW. Lumbar disk disease: Epidemiology. Instr Course Lect 1992; 41:217-223.

261. Wilkinson HA. Introduction: Etiology, diagnosis, and therapy. InThe Failed Back Syndrome. Etiology and therapy, Second Edition. Springer-Verlag, New York, 1992, pp 1-3.

262. Wilkinson HA. The role of improper surgery in the etiology of the failed back syndrome. In: The Failed Back Syndrome. Etiology and therapy, SecondEdition. Springer-Verlag, New York, 1992, pp 4-12.

263. Law JD, Lehman RAW, Kirch WM. Reoperation after lumbar intervertebral disc surgery. JNeurosurg 1978; 48:259-263.

264. Biondi J, Greenberg BJ. Redecompression and fusion in failed back syndrome patients.J Spinal Disord 1990; 3:362-369.

265. Turner JA, Ersek M, Herron L et al. Surgery for lumbar spinal stenosis, attempted meta-analysis of the literature. Spine 1992; 17:1-8.

266. Waddell G, Kummel EG, Lotto WN et al. Failed lumbar disc surgery and repeat surgery following industrial injury. J Bone Joint Surg (Am) 1979; 61:201-207.

267. Ross JS, Robertson JT, Frederickson RCA et al. Association between peridural scar and recurrent radicular pain after lumbar discectomy: Magnetic resonance evaluation. Neurosurgery 1996; 38:855-863.

268. Fritsch EW, Heisel J, Rupp S. The failed back surgery syndrome. Reasons, intraoperative findings, and longterm results: A report of 182 operative treatments. Spine 1996; 21:626-633.

269. Parke WW, Watanable R. Adhesions of the ventral lumbar dura. Adjunct source of discogenic pain?Spine 1990; 15:300-303.

270. Quiles M, Marchisello PJ, Tsairis P. Lumbar adhesive arachnoiditis: Ethological and pathological aspects. Spine 1978; 3:45-50.

271. Benoist M, Ficat C, Baraf P, et al. Post operative lumbar epiduroarachnoiditis: Diagnostic and therapeutic aspects. Spine 1980; 5:432-436.

272. Fager CA, Freidberg SR. Analysis of failures and poor results of lumbar spine surgery. Spine 1980; 5:87-94.
273. Hanley EN, Shapiro DE. The development of low back pain after excision of a lumbar disc. JBone Joint Surg 1989; 71A:719-721.

274. Frymoyer JW. Magnitude of the problem. In Weinstein J, Weisel SW (eds). The Lumbar Spine. WB Saunders, Philadelphia, 1990, pp 32-38.

275. LaRocca H. Failed lumbar surgery. Principles of management. In Weinstein J, Wiesel S (eds). The Lumbar Spine. WB Saunders Company, Philadelphia, 1990, pp 872-881.

276. Rutkow IM. Orthopaedic operations in the United States, 1979-1983. J Bone Joint Surg 1986; 68A:716719.

277. Hanley EN Jr. The cost of surgical intervention for lumbar disc herniation. In Weinstein JN (ed). Clinical efficacy and outcome in the diagnosis and treatment oflow back pain . Raven Press, New York, 1992, pp 125-133.

278. Burton CV. Causes of failure of surgery on the lumbar spine: Ten-year follow up. Mt Sinai J Med 1991; 58:183-187.

279. Burton CV, Kirkaldy-Willis WH, Yong-Hing K et al. Causes of failure of surgery on the lumbar spine. Clin Orthop 1981; 157:191-199.

280. North RB, Campbell JN, James CS et al. Failed back surgery syndrome: 5 year follow-up in 102 patients undergoing repeated operation. Neurosurgery 1991; 28:685-691.

281. Saal JS, Saal JA, Yurth EF. Nonoperative management of herniated cervical intervertebral disc with radiculopathy. Spine 1996; 21:1877-1883.

282. Turner JA, Ersek, Herron L et al. Patient outcomes after lumbar spinal fusions. JAMA 1992; 268:907-911.

283. Keller RB, Atlas SJ, Soule DN et al. Relationship between rates and outcomes of operative treatment for lumbar disc herniation and spinal stenosis. J Bone Joint Surg 1999; 81A(6):752-762.

284. Porchet F, Vader JP, Larequi-Lauber T et al. The assessment of appropriate indications for laminectomy. J Bone Joint Surg 1999;81B(62):234-239.

285. Davis H. Increasing rates of cervical and lumbar spine surgery in the United States, 1979-1990. Spine 1994; 19:1117-1124.

286. Postacchini F. Results of surgery compared with conservative management for lumbar disc herniations. Spine 1996; 21:1383-1387.

287. Airaksinen O, Herno A, Turunen V et al. Surgical outcome of 438 patients treated surgically for lumbar spine stenosis. Spine 1997; 22:2278-2282.

288. Klekamp J, McCarty E, Spengler DM. Results of elective lumbar discectomy for patients involved in the workers' compensation system. J Spinal Disord 1998; 11:277-282.

289. Nachemson AL. Failed back surgery syndrome is syndrome of failed back surgeons. Pain Clinic 1999; 11:271-284. 
290. Waddell G, Gibson NA, Grant I. Surgical treatment of lumbar disc prolapse and degenerative lumbar disc disease. In Nachemson AL, Jonsson E (eds). Neck and Back Pain. Lippincott Williams \& Wilkins, Philadelphia, 2000, pp 305-325.

291. Gibson JNA, Grant IC, Waddell G. The Cochrane review of surgery for lumbar disc prolapse and degenerative lumbar spondylosis. Spine 1999; 24:18201832.

292. Keskimaki I, Seitsalo S, Osterman H et al. Reoperations after lumbar disc surgery. A populationbased study of regional and interspecialty variations. Spine 2000; 25:1500-1508.

293. Weber H. Lumbar disc herniation: Controlled, prospective study with ten years of observation. Spine 1983; 8:131-140.

294. Dreyfuss P, Tibiletti C, Dreyer S et al. Thoracic zygapophyseal joint pain: A review and description of an intra-articular block techniques. Pain Digest 1994; 4:46-54.

295. Lord SM, Barnsley L, Wallis BJ et al. Third occipital nerve headache. A prevalence study. J Neurol Neurosurg Psychiatry 1994; 57:1187-1190.

296. Bovim G, Berg R, Dale LG. Cervicogenic headache: Anesthetic blockades of cervical nerves (C2-C5) and facet joint (C2/C3). Pain 1992; 49:315-320.

297. Fukusaki M, Tsuji K, Fujie T et al. The effects of blockade of the facet joint of the atlas and axis upon muscle tension headaches. Headache 1990; 4:312-317.

298. Ogoke BA. The management of the atlanto-occipital and atlanto-axial joint pain. Pain Physician 2000; 3:289-293.

299. McCormick CC. Arthrography of the atlanto-axial (C1-C2) joints: Technique and results. J Intervent Radiol 1987; 2:9.

300. Melton LJ, Lane AW, Cooper C et al: Prevalence and incidence of vertebral deformities. Osteoporosis Int 1993; 3:113-119.

301. Buss DD, Wright RW. Etiology, diagnosis, and treatment of Paget's disease. Curr Opinion Orthop 1994; 5:26-32.

302. Tolli TC, Cammisa FP Jr, Lane JM et al. Metastatic disease of the spine. Semin Spine Surg 1995;7:277287.

303. Borenstein D. Approach to the diagnosis and management of medical low back pain. Semin Spine Surg 1990; 2:80-85.

304. Bernini PM, Simeone FA. Reflex sympathetic dystrophy associated with lumbar disc herniation. Spine 1981; 6:180-184.

305. Manchikanti L. Evaluation and management services in interventional pain practice: Doing it right! Pain Physician 2000; 3:322-341.

306. Waddell G, Turk DC. Clinical assessment of low back pain. In Turk DC, Melzack R (eds). Handbook of pain assessment. First Edition. The Guilford Press, New
York, 1992, pp 15-36.

307. Cathelin F. Mode d'action de a cocaine injete daus l'escapte epidural par le procede du canal sacre. Comptes Rendies des Senaces de la Societe de Biologic et de ses Filliales, 1901;43:487.

308. Pasquier NM, Leri D. Injection intra-et extradurales de cocaine a dose minime daus le traitment de la sciatique. Bull Gen Ther 1901; 142:196.

309. Sicard MA. Les injections medicamenteuse extraduraqles per voie saracoccygiene. Comptes Renues des Senances de la Societe de Biolgie et de ses Filliales, 1901; 53:396.

310. Caussade G, Queste P. Traitement de al neuralgie sciatique par la mèthode de Sicard. Résultats favorables même dans les cas chroniues par la cocaïne à doses élevées et répétées à intervalles raproches. Bull Soc Med Hosp Paris 1909; 28:865.

311. Pages E. Anesthesia metamerica. Rev Sanid Mil Madr 1921; 11:351-385.

312. Viner N. Intractable sciatica-The sacral epidural injection-An effective method of giving pain relief. Can Med Asso J 1925; 15:630-634.

313. Evans W. Intrasacral epidural injection in the treatment of sciatica. Lancet 1930; 2:1225-1229.

314. Rovenstein EA, Wertheim HM. Therapeutic nerve block. JAMA 1941; 117:1599-1603.

315. Robechhi A., Capra R. L'idrocortisone (composto F). Prime esperienze cliniche in campo reumatologico. Minerva Med 1952; 98:1259-1263.

316. Lievre JA, Block-Michel H, Attali P. L'injection transscree etude clinique et radiologrique. Bull Soc Med Hop 1957; 73:1110-1118.

317. Goebert HW, Jallo SJ, Gardner WJ et al. Painful radiculopathy treated with epidural injections of procaine and hydrocortisone acetate results in 113 patients. Anesth Analg 1961;140:130-134.

318. Steindler A. Luck JV. Differential diagnosis of pain in the low back: Allocation of the source of the pain by the procaine hydrochloride method. JAMA 1938; 110:106-113.

319. Wertheim HM, Rovenstine EA. Suprascapular nerve block. Anesthesiology 1941; 2:541.

320. Sluijter ME, Koetsveld-Baart CC. Interruption of pain pathways in the treatment of cervical syndrome. Anaesthesia 1980; 35:302-307.

321. Hildebrandt J, Argyrakis A. Percutaneous nerve block of the cervical facets - A relatively new method in the treatment of chronic headache and neck pain. Man Med 1986; 2:48-52.

322. Okada K. Studies on the cervical facet joints using arthrography of the cervical facet joint. JJpn Orthop Assoc 1981; 55:563-580.

323. White JC. Diagnostic novocaine block of the sensory and sympathetic nerves. Am J Surg 1930; 9:264.

324. Bonica JJ. The management of Pain. Lea \& Febiger, Philadelphia, 1953.

325. Fox AJ, Melzack R. Transcutaneous electrical stimu- 
lation to acupuncture. Comparison of treatment of low back pain. Pain 1976; 2:141-148.

326. Fowler RJ, Blackwell GJ. Anti-inflammatory steroid induced biosynthesis of a phospholipase A 2 inhibitor which prevents prostaglandin generation. Nature 1979; 278:456-459.

327. Devor M, Govrin-Lippmann R, Raber P. Corticosteroids suppress ectopic neural discharges originating in experimental neuromas. Pain 1985;22:127-137.

328. Johansson A, Hao J, Sjolund B. Local corticosteroid application blocks transmission in normal nociceptor C-fibers. Acta Anaesthesiol Scand 1990; 34:335-338.

329. Faber LE, Wakim NG, Duhring JL. Evolving concepts in the mechanism of steroid action: Current developments. Am J Obstet Gynecol 1987; 156:14491458.

330. Olmarker K, Byrod G, Cornefijord M et al. Effects of methylprednisolone on nucleus pulposus-induced nerve root injury. Spine 1994; 19:1803-1808.

331. Nicol GD, Klingberg DK, Vasko MR. Prostaglandin $\mathrm{E}_{2}$ enhances calcium conductance and stimulates release of substance $\mathrm{P}$ in avian sensory neurons. $J$ Neurosci 1992; 12:1917-1927.

332. Hua SY, Chen YZ. Membrane receptor-mediated electrophysiological effects of glucocorticoid on mammalian neurons. Endocrinology 1989; 124:687-691.

333. Coderre $\mathrm{T}$. Contribution of protein kinase $\mathrm{C}$ to central sensitization and persistent pain following tissue injury. Neurosci Lett 1992; 140:181-184.

334. Hayashi N, Weinstein JN, Meller ST et al. The effect of epidural injection of betamethasone or bupivacaine in a rat model of lumbar radiculopathy. Spine 1998; 23:877-885.

335. Lee HM, Weinstein JN, Meller ST et al. The role of steroids and their effects on phospholipase $\mathrm{A}_{2}$. An animal model of radiculopathy. Spine 1998;23:11911196.

336. Minamide A, Tamaki T, Hashizume Het al. Effects of steroids and lipopolysaccharide on spontaneous resorption of herniated intervertebral discs. An experience study in the rabbit. Spine 1998;23:870-876.

337. Kingery WS, Castellote JM, Maze M. Methylprednisolone prevents the development of autotomy and neuropathic edema in rats, but has no effect on nociceptive thresholds. Pain 1999; 80:555-566.

338. Manchikanti L. The value and safety of steroids in neural blockade: Part 1. AJPM 2000; 10:69-78.

339. Manchikanti L. The value and safety of steroids in neural blockade: Part 2. AJPM 2000; 10:121-133.

340. Johansson A, Bennett GJ. Effect of local methylprednisolone on pain in a nerve injury model. A pilot study. Reg Anesth 1997; 22:59-65.

341. Li Yue-Ming, Wingrove DE, Too HP et al. Local anesthetics inhibit substance $\mathrm{P}$ binding and evoked increases in intracellular $\mathrm{Ca}^{2+}$. Anesthesiology 1995:82:166-173

342. Bonica JJ, Backup PH, Anderson CE. Peridural block, an analysis of 3,637 cases. A review. Anesthesiology 1957; 18:723-734.

343. Fink BR, Cairns AM. Differential use-dependent (frequency-dependent) effects in single mammalian axons: Data and clinical considerations. Anesthesiology1987; 67:477-484.

344. Deyo RA, Rainville J, Kent DL. What can the history and physical examination tell us about low back pain? JAMA 1992; 268:760-765.

345. Haldeman $\mathrm{S}$. Failure of the pathological model to predict back pain. Spine 1990; 15:718-732.

346. Schwarzer AC, Scott AM, Wang SC et al. The role of bone scintigraphy in chronic low back pain: A comparison of SPECT and planar images and zygapophysial joint injection. Aust NZJ Med 1992; 22:185.

347. Schwarzer AC, Wang SC, O'Driscoll Det al. The ability of computed tomography to identify a painful zygapophysial joint in patients with chronic low back pain. Spine 1995; 20:907-912.

348. Deyo RA. Fads in the treatment of low back pain. $N$ Engl J Med 1991; 325:1039-1040.

349. Saal JA. Natural history and non-operative treatment of lumbar disc herniation. Spine 1996;21:25-35.

350. Thelander U. Fagerlung M., Friberg S et al. Straight leg raising test vs radiologic size, shape, and position of lumbar disc herniations. Spine 1992; 17:395-389.

351. Jackson RP, Jacobs RR, Montesano PX. Facet joint injection in low back pain. A prospective study. Spine 1988; 13:966-971.

352. Drew B, Bhandari M, Kulkarni AV et al. Reliability in grading the severity of lumbar spinal stenosis. $J$ Spinal Disord 2000; 13:253-258.

353. O'Neill C, Derby R, Kenderes L. Precision injection techniques for diagnosis and treatment of lumbar disc disease. Seminars in Spine Surgery 1999; 11:104-118.

354. Grönblad M, Virri J, Seitsalo S et al. Inflammatory cells, motor weakness, and straight leg raising in transligamentous disc herniations. Spine 2000; 25:2803-2807.

355. Jensen IB, Bodin L, Ljungqvist T et al. Assessing the needs of patients in pain: A matter of opinion? Spine 2000; 25:2816-2823.

356. Carragee EJ. The prevalence and clinical features of internal disc disruption (IDD) in patients with chronic low back pain. Spine 1996; 21:776.

357. Schwarzer A, Bogduk N. The prevalence and clinical features of internal disc disruption (IDD) in patients with chronic low back pain. Spine 1996; 21:776-777.

358. Dreyfuss P, Michaelsen M, Pauza K et al. The value of medical history and physical examination in diagnosing sacroiliac joint pain. Spine 1996; 21:25942602.

359. Slipman CW, Jackson HB, Lipetz JL et al. Sacroiliac joint pain referral zones. Arch Phys Med Rehab 2000; 81:334-337.

360. Meijne W, Van Neerbos K, Aufdemkampe G et al. 
Intraexaminer and interexaminer reliability of the Gillet test. J Man Phys Ther 1999;22:4-9.

361. Slipman CW, Sterenfeld EB, Chou LH et al. The predictive value of provocative sacroiliac joint stress maneuvers in the diagnosis of sacroiliac joint syndrome. Arch Phys Med Rehab 1998; 79:288-292.

362. Broadhurst NA, Bond MJ. Pain provocation tests for the assessment of sacroiliac joint dysfunction. J Spin Disord 1998; 11:341-345.

363. Ebraheim NA, Mekhail AO, Wiley WF et al. Radiology of the sacroiliac joint. Spine 1997; 22:869-876.

364. Espeland A, Korsbrekke K, Albreksten G et al. Observer variation in plain radiography of the lumbosacral spine. Brit J Rad 1998; 71:366-375.

365. Vogel JB III, Brown WH, Helms CA et al. The normal sacroiliac joint: A CT study of asymptomatic patients. Radiol 1984; 151:433-437.

366. Greenman PE. Clinical aspects of sacroiliac function in walking. J Man Med 1990; 5:25-130.

367. Slipman CW, Sterenfield EB, Chou LH et al. The value of radionuclide imaging in the diagnosis of sacroiliac joint syndrome. Spine 1996; 21:2251-2254.

368. Maigne JY, Boulahdour H, Charellier G. Value of quantitative radionuclide bone scanning in the diagnosis of sacroiliac joint syndrome in 32 patients with low back pain. Eur Spine J 1998; 7:328-331.

369. Goldberg R, Genant H, Shimshak R et al. Applications and limitations of quantitative sacroiliac joint scintigraphy. Radiology 1978;128:683-686.

370. Lantto T. The scintigraphy of sacroiliac joints: A comparison of 99-m Tc-VPB and 99mTc-MDP. EurJNucl Med 1990; 16:677-681.

371. Hanly JG, Mitchell MJ, Barnes DC et al. Early recognition of sacroiliitis by magnetic resonance imaging and single photon emission computed tomography. $J$ Rheum 1994; 21:2088-2095.

372. Barnsley L, Lord S, Bogduk N. Comparative local anesthetic blocks in the diagnosis of cervical zygapophysial joints pain. Pain 1993; 55:99-106.

373. Lord SM, Barnsley L, Bogduk N. The utility of comparative local anesthetic blocks versus placebo-controlled blocks for the diagnosis of cervical zygapophysial joint pain. Clin J Pain 1995; 11:208213.

374. Schwarzer AC, Aprill CN, Derby R et al. The falsepositive rate of uncontrolled diagnostic blocks of the lumbar zygapophysial joints. Pain 1994; 58:195-200.

375. Barnsley L, Bogduk N. Medial branch blocks are specific for the diagnosis of cervical zygapophyseal joint pain. Reg Anesth 1993; 18:343-350.

376. Dreyfuss P, Schwarzer AC, Lau P et al. Specificity of lumbar medial branch and L5 dorsal ramus blocks: A computed tomography study. Spine 1997;22:895-902.

377. North RB, Han M, Zahurak Met al. Radiofrequency lumbar facet denervation: Analysis of prognostic factors. Pain 1994; 57:77-83.
378. Kaplan M, Dreyfus P, Halbrook B et al. The ability of lumbar medial branch blocks to anesthetize zygapophysial joint. Spine 1998; 23:1847-1852.

379. Nash TP. Facet joints. Intra-articular steroids or nerve blocks? Pain Clinic 1990; 3:77-82.

380. Marks RC, Houston T, Thulbourne T. Facet joint injection and facet nerve block. A randomized comparison in 86 patients with chronic low back pain. Pain 1992; 49:325-328.

381. Bogduk N. Diskography. APSJ 1994; 3:149-154.

382. Esses SI. The diskography dilemma. APSJ 1994; 3:155-156.

383. Campbell JN, Belzberg. Use of disk distension to diagnose pain of spinal origin. APSJ 1994;3:157-159.

384. Roofe PG. Innervation of the anulus fibrosus and posterior longitudinal ligament. Arch Neurol Psych 1940; 44:100-103.

385. Lindblom K. Protrusions of the discs and nerve compression in the lumbar region. Acta Radiol Scand1944; 25:195-212.

386. Hirsch C. An attempt to diagnose level of disc lesion clinically by disc puncture. Acta Orthop Scand 1948; 18:131-140.

387. Shinomiya K, Nakao N, Shindoh K et al. Evaluation of cervical discography in pain origin and provocation. J Spinal Disord 1993; 6:422-426.

388. Simmons E. An evaluation of discography in the localization of symptomatic levels in discogenic diseases of the spine. Clin Orthop 1975; 108:57-59.

389. Schellhas KP, Smith MD, Gundry CR et al. Cervical discogenic pain. Spine 1996; 21:300-311.

390. Holt E. Fallacy of cervical discography. JAMA 1964; 188:799-801.

391. Moneta GB, Videman T, Kaivanto K et al. Reported pain during lumbar discography as a function of anular ruptures and disc degeneration: A re-analysis of 833 discograms. Spine 1994; 17:1968-1974.

392. Walsh TR, Weinstein JN, Spratt KP et al. Lumbar discography in normal subjects. J Bone Joint Surg 1990; 72A:1081-1088.

393. Bogduk N. The argument for discography. Neurosurgery Quarterly 1996; 6:152-153.

394. Bogduk N, Modic MT. Controversy: Lumbar discography. Spine 1996; 21:402-404.

395. The Executive Committee of the North American Spine Society. Position statement on discography. Spine 1988; 13:1343.

396. Slipman CW. Discography. In Gonzalez EG, Materson RS (eds). The Nonsurgical Management of Acute Low Back Pain. Demos vermande, New York, 1997, pp 103-114.

397. Guyer RD, Ohnmeiss DD. Contemporary concepts in spine care. Lumbar discography. Position statement from the North American Spine Society and Therapeutic Committee. Spine 1995; 18:2048-2059.

398. Carragee EJ, Tanner CM, Yang B et al. False-Positive 
findings on lumbar discography. Spine 1999;24:25422547.

399. Carragee E, Tanner C, Khurana S et al. The rates of false-positive lumbar discography in select patients without low back symptoms. Spine 2000; 25:13731381.

400. Carragee EJ, Chen Y, Tanner CM et al. Provocative discography in patients after limited lumbar discectomy. Spine 2000; 25:3065-3071.

401. Carragee EJ, Paragioudakis SJ, Khurana S. Lumbar high-intensity zone and discography in subjects without low back problems. Spine 2000; 25:2987-2992.

402. Carragee E, Chen Y, Tanner C et al. Can discography cause long-term back symptoms in previously asymptomatic subjects? Spine 2000;25:1803-1808.

403. Bogduk N. Prelude to a discussion on discography, towards an acceptable and convincing standard of practice. ISIS $8^{\mathrm{TH}}$ Annual Scientific Meeting, San Francisco, Sept. 2000.

404. Derby R, Howard M, Grant J et al. The ability of pressure controlled discography to predict surgical and nonsurgical outcome. Spine 1999;24:364-371.

405. Macnab I. Negative disc exploration: An analysis of the causes of nerve root involvement in sixty-eight patient. J Bone Joint Surg [AM] 1971;53A:58915903.

406. Slipman CW. Diagnostic nerve root blocks. In: the nonsurgical management of acute low back pain. Gonzalez EG, Materson RS (eds). Demos vermande, New York 1997: pp 115-122.

407. Schutz H, Lougheed WM, Wortzman G et al. Intervertebral nerve-root in the investigation of chronic lumbar disc disease. Can J Surg 1973; 16:217-221.

408. Krempen JF, Smith BS. Nerve root injection: A method for evaluating the etiology of sciatica. J Bone Joint Surg [AM] 1974; 56A:1435-1444.

409. Tajima T, Furukawa K, Kuramochi E. Selective lumbosacral radiculography and block. Spine 1980; 1:6877.

410. Haueisen DC, Smith BS, Myers SR et al. The diagnostic accuracy of spinal nerve injection studies. Clin Orthop Rel Res 1985; 198:179-183.

411. Dooley JF, McBroom RJ, Taguchi T et al. Nerve root infiltration in the diagnosis of radicular pain. Spine 1988; 13:79-83.

412. Stanley D, McLaren MI, Euinton HA et al. A prospective study of nerve root infiltration in the diagnosis of sciatica: A comparison with radiculography, computed tomography, and operative findings. Spine 1990; 6:540-543.

413. Derby R, Kine G, Saal JA et al. Response to steroid and duration of radicular pain as predictors of surgical outcome. Spine 1992; 17 (Suppl):176-183.

414. Castro WH, van Akkerveeken PF. Der diagn ostische Wert der selektiven lumbalen Nervenwurzelblockde. A orthop Ihre Grenzgeb 1991; 129:376-379.

415. Kikuchi S, Hasue M, Nishiyama K. Anatomic and clinical studies of radicular symptoms. Spine 1984; 9:23-30.

416. Van Akkerveeken PF. The diagnostic value of nerve root sheath infiltration. Acta Orthop Scand 1993; 64:61-63.

417. Herron LD. Selective nerve root block in patient selection for lumbar surgery: Surgical results. JSpinal Disord 1989; 2:75-79.

418. Hoppenstein R. A new approach to the failed back syndrome. Spine 1980; 5:371-379.

419. Nachemson A. Newest knowledge of low back pain: A critical look. Clin Orthop Rel Res 1992; 279:8-20.

420. Van Akkerveeken PF. Lateral stenosis of the lumbar spine. Thesis. University of Utrecht, 1989.

421. Tulder MWV, Goossens M, Waddell G et al. Conservative treatment of chronic low back pain. In Nachemson AL, Jonsson E (eds). Neck and Back Pain. Lippincott Williams \& Wilkins, Philadelphia, 2000, pp 271-304.

422. Tulder MWV, Osteolo R, Vlaeyen JWS et al. Behavioral treatment for chronic low back pain. A systematic review within the framework of the Cochrane back review group. Spine 2000; 25:2688-2699.

423. Hazard RG, Reid S, Haugh LD et al. A controlled trial of an educational pamphlet to prevent disability after occupational low back injury. Spine 2000; 25:1419-1423.

424. Wassell JT, Gardner LI, Landsittel DP et al. A prospective study of back belts for prevention of back pain and injury. JAMA 2000; 284:2727-2732.

425. Tulder MWV, Malmivaara A, Esmail R. Exercise therapy for low back pain. A systematic review within the framework of the Cochrane collaboration back review group. Spine 2000; 25:2784-2796.

426. Hansson TH, Hansson EK. The effects of common medical interventions on pain, back function, and work resumption in patients with chronic low back pain. Spine 2000; 25:3055-3064.

427. Ezzo J, Berman B, Hadhazy VA et al. Is acupuncture effective for the treatment of chronic pain? A systematic review. Pain 2000; 86:217-225.

428. Tulder MWV, Cherkin DC, Berman B et al. The effectiveness of acupuncture in the management of acute and chronic low back pain. A systematic review within the framework of the Cochrane Collaboration Back Review Group. Spine 1999; 24:1113-1123.

429. Hagen KB, Hilde G, Jamtvedt $\mathrm{G}$ et al. The Cochrane review of bed rest for acute low back pain and sciatica. Spine 2000; 25:2932-2939.

430. Altmaier EM, Lehmann T, Russell DW et al. The effectiveness of psychological interventions for the rehabilitation of low back pain: A randomized controlled trial evaluation. Pain 1992; 49:329-335.

431. Bush C, Ditto B, Feurstein M. A controlled evaluation of paraspinal EMG biofeedback in the treatment of chronic low back pain. Health Psychol 1985;4:307321. 
432. Donaldson S, Romney D, Donaldson M et al. Randomized study of the application of single motor unit biofeedback training to chronic low back pain.JOccup Rehabil 1994; 4:23-37.

433. Lindström I, Öhlund C, Eek C et al. The effect of graded activity on patients with subacute low back pain: A randomized prospective clinical study with an operant-conditioning behavioral approach. Phys Ther 1992; 72:279-293.

434. Lindström I, Öhlund C, Eek C et al. Mobility, strength, and fitness after a graded activity program for patients with subacute low back pain: A randomized prospective clinical study with a behavioral therapy approach. Spine 1992; 17:641-652.

435. McCauley JD, Thelen MH, Frank RG et al. Hypnosis compared with relaxation in the outpatient management of chronic low back pain. Arch Phys Med Rehabil 1983; 64:548-552.

436. Newton-John T, Spence SH, Schotte D. Cognitivebehavioral therapy versus EMG biofeedback in the treatment of chronic low back pain. Behav Res Ther 1995; 33:691-697.

437. Nouwen A. EMG biofeedback used to reduce standing levels of paraspinal muscle tension in chronic low back pain. Pain 1983; 17:353-360.

438. Rose MJ, Reilly JP, Pennie B et al. Chronic low back pain rehabilitation programs: A study of the optimum duration of treatment and a comparison of group and individual therapy. Spine 1997;22:2246-2253.

439. Koes BW, Assendelft WJJ, Heijden GJMG et al. Spinal manipulation and mobilization for back and neck pain: A blinded review. Br Med J 1991; 303:12981303.

440. Koes BW, Bouter LM, Mameren H V, et al. A blinded randomized clinical trial of manual therapy and physiotherapy for chronic back and neck complaints: Physical outcome measures. $J$ Manipulative Physiol Ther 1992; 1:16-23.

441. Mayer TG, Polatin P, Smith B et al. Spine rehabilitation. Secondary and tertiary nonoperative care. Spine 1995; 20:2060-2066.

442. Teasell RW, Harth M. Functional restoration. Returning patients with chronic low back pain to work-Revolution or fad? Spine 1996; 21:844-847.

443. Van der Windt DAWM, van der Jeijden GJMG, van den Berg SGM et al. Ultrasound therapy for musculoskeletal disorders. A systematic review. Pain 1999; 81:257-271

444. Hurwitz EL, Aker PD, Adams AH et al. Manipulation and mobilization of the cervical spine. A systematic review of the literature. Spine 1996;21:1746-1760.

445. Grant DJ, Bishop-Miller J, Winchester DM, et al. A randomized comparative trial of acupuncture versus transcutaneous electrical nerve stimulation of chronic back pain in the elderly. Pain 1999; 82:9-13.

446. Cassisi JE, Sypert GW, Salamon A et al. Independent evaluation of a multidisciplinary rehabilitation program for chronic low back pain. Neurosurgery1989;25:877883.

447. Robinson JP, Allen T, Fulton LD et al. Perceived efficacy of pain clinics in the rehabilitation of injured workers. Clin J Pain 1998; 14:202-208.

448. Triano JJ, McGregor M, Hondras MA et al. Manipulative therapy versus education programs in chronic low back pain. Spine 1995; 20:469-472.

449. Mayer T, McMahon MJ, Gatchel RJ et al. Socioeconomic outcomes of combined spine surgery and functional restoration in workers' compensation spinal disorders with matched controls. Spine 1998; 23:598606.

450. Wheeler AH, Hanley EN Jr. Nonoperative treatment for low back pain. Rest to restoration. Spine 1995; 20:375-378.

451. Morley S, Eccleston C, Williams A. Systematic review and meta-analysis of randomized controlled trials of cognitive behavior therapy for chronic pain in adults, excluding headache. Pain 1999; 90:1-13.

452. Koes BW, Hoogen HMMV. Efficacy of bed rest and orthoses for low back pain: A review of randomized clinical trials. Eur J Phys Med Rehab 1994; 4:86-93. van Poppel MN, Koes BW, van der Ploeg T et al. Lumbar supports and education for the prevention of low back pain in industry: A randomized controlled trial. JAMA 1998; 279:1789-1794.

454. Smith GW, Robinson RA. The treatment of certain cervical spine disorders by anterior removal of the intervertebral disc and interbody fusion. JBone Joint Surg [AM] 1958; 40:607-623.

455. Parfenchuck T, Janssen M. A correlation of cervical magnetic resonance imaging and discography-computed tomographic discograms. Spine 1994;19:28192825.

456. Vanharanta H, Sachs BL, Spivey MA et al. The relationship of pain provocation to lumbar disc deterioration as seen by CT/discography. Spine 1987; 12:295298.

457. Carette S, Marcoux S, Truchon R et al. A controlled trial of corticosteroid injections into facet joints for chronic low back pain. N Engl J Med 1991;325:10021007.

458. Carrera GF. Lumbar facet joint injection in low back pain and sciatica: Preliminary results. Radiology 1980; 137:665-667.

459. Fairbank JCT, Park WM, McCall IW et al. Apophyseal injection of local anesthetic as a diagnostic aid in primary low back pain syndromes. Spine 1981; 6:598605.

460. Desoutet JM, Gilula LA, Murphy WA et al. Lumbar facet joint injection: Indication, technique, clinical correlation, and preliminary results. Radiology 1982; 145:321-325.

461. Raymond J, Dumas JM. Intra-articular facet block: Diagnostic tests or therapeutic procedure? Radiology 1989; 151:333-336. 
462. Lippitt AB. The facet joint and its role in spine pain. Management with facet joint injections. Spine 1984; 9:746-750.

463. Lau LS, Littlejohn GO, Miller MH. Clinical evaluation of intra-articular injections for lumbar facet joint pain. Med J Aust 1985; 143:563-565.

464. Lewinnek GE, Warfield CA. Facet joint degeneration as a cause of low back pain. Clin Orthop 1986; 213:216-222.

465. Murtagh FR. Computed tomography and fluoroscopy guided anesthesia and steroid injection in facet syndrome. Spine 1988; 13:686-689.

466. Helbig T, Lee CK. The lumbar facet syndrome. Spine 1988; 13:61-64.

467. Moran R, O'Connell D, Walsh MG. The diagnostic value of facet joint injections. Spine 1986; 12:14071410.

468. Lilius G, Laasonen EM, Myllynen P et al. Lumbar facet joint syndrome. A randomized clinical trial. J Bone Joint Surg (Br) 1989; 71:681-684.

469. Lynch MC, Taylor JF. Facet joint injection for low back pain. A clinical study. J Bone Joint Surg (Br) 1986; 68:138-141.

470. Dory MA. Arthrography of the lumbar facet joints. Radiology 1981; 140:23-27.

471. Mironer YE, Somerville JJ. Protocol for diagnosis and treatment of facet joint pain syndrome. A modified three-step approach. Pain Digest 1999; 9:188-190.

472. Dreyfuss P. Dreyer S. Lumbar facet joint injections. In Gonzalez EG, Materson RS (eds). The Nonsurgical Management of Acute Low Back Pain. Demos vermande, New York 1997, pp 123-136.

473. Jackson RP. The facet syndrome. Myth or reality? Clin Orthop 1992; 279:110-121.

474. Nachemson AL, Vingard E. Assessment of patients with neck and back pain: A best-evidence synthesis. In Nachemson AL, Jonsson E (eds). Neck and Back $P a i n$. The scientific evidence of causes, diagnosis and treatment. Lippincott Williams \& Wilkins, New York, 2000, pp 189-236.

475. Barnsley L, Lord SM, Wallis BJ et al. Lack of effect of intra-articular corticosteroids for chronic pain in the cervical zygapophyseal joints. N Engl J Med 1994; 330:1047-1050.

476. Roy DF, Fleury J, Fontaine SB et al. Clinical evaluation of cervical facet joint infiltration. Can Assoc Radiol J 1988; 39:118-120.

477. Dusault DG, Nicolet VM. Cervical facet joint arthrography. J Can Assoc Radiol 1985; 36:79-80.

478. Wedel DJ, Wilson PR. Cervical facet arthrography. Reg Anesth 1985; 10:7-11.

479. Dory MA. Arthrography of the cervical facet joints. Radiology 1983; 148:379-382.

480. Hove B, Glydensted C. Cervical analgesia facet joint arthrography. Neuroradiology 1990; 32:456-459.

481. Manchikanti L, Pampati V, Bakhit CE et al. Effective- ness of lumbar facet joint nerve blocks in chronic low back pain: A randomized clinical trial. Pain Physician 2001; 4: 101-117.

482. Lord SM, Barnsley L, Wallis BJ et al. Percutaneous radio-frequency neurotomy for chronic cervical zygapophyseal-joint pain. $N$ Eng $J$ Med 1996; 335:1721-1726.

483. Shealy CN. Percutaneous radiofrequency denervation of spinal facets. JNeurosurg 1975;43:448-451.

484. Shealy CN. Facet denervation in the management of back sciatic pain. Clin Orthop 1976; 115:157-164.

485. Lord SM, Barnsley L, Bogduk N. Percutaneous radiofrequency neurotomy in the treatment of cervical zygapophyseal joint pain: a caution. Neurosurgery 1995; 35:732-739.

486. McDonald GJ, Lord SM, Bogduk N. Long-term follow-up of patients treated with cervical radiofrequency neurotomy for chronic neck pain. Neurosurgery 1999; 45:61-68.

487. Van Kleef M, Barendse GAM, Kessels A et al. Randomized trial of radiofrequency lumbar facet denervation for chronic low back pain. Spine 1999; 24:19371942.

488. Dreyfuss P, Halbrook B, Pauza K et al. Efficacy and validity of radiofrequency neurotomy for chronic lumbar zygapophysial joint pain. Spine 2000; 25:12701277.

489. Van Kleef M, Liem L, Lousberg $\mathrm{R}$ et al. Radiofrequency lesions adjacent to the dorsal root ganglion for cervicobrachial pain. A prospective double blind randomized study. Neurosurgery 1996;38:1-6. 490. Sluijter ME, Mehta M. Treatment of chronic back and neck pain by percutaneous thermal lesions. In Lipton S (ed). Persistent Pain, Modern Methods of Treatment. Academic Press, London, 1981, pp 141-179.

491. Sluijter ME. The use of radiofrequency lesions of the communicating ramus in the treatment of low back pain. In Racz GB (ed). Techniques of Neurolysis. Kluwer Academic Publishers; Boston, 1989, pp 145160.

492. Sluijter ME. The use of radiofrequency lesions for pain relief in failed back patients. Int Disabil Studies 1988; 10:37-43.

493. Mehta M, Sluijter ME. The treatment of chronic pain. Anesthesia 1979; 34:768-775.

494. Rashbaum RF. Radiofrequency facet denervation. A treatment alternative in refractory low back pain with or without leg pain. Orthop Clin North Am 1983; 14:569-575.

495. Banerjee T, Pittman HH. Facet rhizotomy. Another armamentarium for treatment of low backache. $N C$ Med J 1976; 37:354-360.

496. Burton CV. Percutaneous radiofrequency facet denervation. Appl Neurophysiol 1976/77; 39:80-86.

497. Ignelzi RJ, Cummings TW. A statistical analysis of percutaneous radiofrequency lesions in the treatment of chronic low back pain and sciatica. Pain 1980; 
8:181-187.

498. Katz SS, Savitz MH. Percutaneous radiofrequency rhizotomy of the lumbar facets. Mt Sinai J Med 1986; 7:523-525.

499. Koning HM, Mackie DP. Percutaneous radiofrequency facet denervation in low back pain. Pain Clinic 1994; 7:199-204.

500. Oudenhoven RC. The role of laminectomy, facet rhizotomy, and epidural steroids. Spine 1979; 4:145-147.

501. Savitz MH. Percutaneous radiofrequency rhizotomy of the lumbar facets. Ten years experience. MtSinaiJ Med 1991; 58:177-178.

502. Schaerer JP. Treatment of prolonged neck pain by radiofrequency facet rhizotomy. JNeurol Orthop Med Surg 1988; 9:74-76.

503. Vervest ACM, Stolker RJ. The treatment of cervical pain syndromes with radiofrequency procedures. Pain Clinic 1991; 4:103-112.

504. Van Kleef AR, Spaans F, Digemavs W et al. Effects and side effects of percutaneous thermal lesions of the dorsal root ganglion in patients with cervical pain syndromes. Pain 1993; 52:49-53.

505. Bogduk N, Macintosh J, Marsland A. Technical limitations to the efficacy of radiofrequency neurotomy for spinal pain. Neurosurgery 1987; 20:529-535.

506. Bogduk N, Long DM. Percutaneous lumbar medial branch neurotomy. A modification of facet denervation. Spine 1980; 5:193-200.

507. Onofrio BM, Campa HK. Evaluation of rhizotomy. Review of 12 years experience. J Neurosurg 1972; 36:151-155.

508. Schaerer JP. Radiofrequency facet rhizotomy in the treatment of chronic neck and low back pain. Int Surg 1978; 63:53-59.

509. Ogsbury JS, Simon RH, Lehman RAW. Facet "denervation" in the treatment of low back syndrome. Pain 1977; 3:257-263.

510. Gallagher J, Vadi PLP, Wesley JR. Radiofrequency facet joint denervation in the treatment of low back pain - A prospective controlled double-blind study to assess efficacy. Pain Clinic 1994; 7:193-198.

511. King JS, Lagger R. Sciatica viewed as a referred pain syndrome. Surg Neurol 1976; 5:46-50.

512. Slivers RH. Lumbar percutaneous facet rhizotomy. Spine 1990; 15:36-40.

513. Brechner T. Percutaneous cryogenic neurolysis of the articular nerve of Luschka. Reg Anesth 1981; 6:1822.

514. Wallis BJ, Lord SM, Bogduk N. Resolution of psychological distress of whiplash patients following treatment by radiofrequency neurotomy: A randomized, double-blind, placebo-controlled trial. Pain 1997; 73:15-22.

515. Brown JH. Pressure caudal anesthesia and back manipulation. Northwest Med (Seattle) 1960; 59:905909.

516. Benzon HT. Epidural steroid injections. Pain Digest
1992; 1:271-280.

517. McQuay HJ, Moore RA. Epidural corticosteroids for sciatica. An Evidence-Based Resource for Pain Relief. Oxford University Press, Oxford, New York, 1998; pp 216-218.

518. White AH, Derby R, Wynne G. Epidural injections for diagnosis and treatment of low back pain. Spine 1980; 5:78-86.

519. Weinstein SM, Herring SA, Derby R. Epidural steroid injections. Spine 1995; 20:1842-1846.

520. Savolaine ER, Pandya JB, Greenblat SHet al. Anatomy of the human lumbar epidural space: New insights using CT-epidurography. Anesthesiology 1988; 68:217220.

521. Hogan QH. Epidural anatomy examined by cryomicrotome section. Influence of age, vertebral level and disease. Reg Anesth 1996;21:295-306.

522. Winnie AP, Hartman JT, Meyers HL et al. Pain Clinic II: Intradural and extradural corticosteroids for sciatica. Anesth Analg 1972;51:990-1003.

523. Sehgal AD, Tweed DC, GardnerWS. Laboratory studies after intrathecal corticosteroids. Arch Neurol1963; 9:64-68.

524. Swerdlow M, Sayle-Creer W. A study of extradural medication in the relief of three lumbosciatic syndromes. Anaesthesia 1970; 25:341-345.

525. Manchikanti L, Bakhit CE, Pampati V. Role of epidurography in caudal neuroplasty. Pain Digest 1998; 8:277-281.

526. Saberski LR, Kondamuri S, Osinubi OYO. Identification of the epidural space: Is loss of resistance to air a safe technique? Regional Anesthesia 1997; 22:315.

527. Rathmell JP, Song T, Torian D et al. Lumbar epidurography. Reg Anesth Pain Med 2000;25:540545.

528. Renfrew DL, Moore TE, Kathol MH et al. Correct placement of epidural steroid injections: Fluoroscopic guidance and contrast administration. Amer $J$ Neuroradiol 1991; 12:1003-1007.

529. Stewart HD, Quinnell RC, Dann N. Epidurography in the management of sciatica. Br J of Rheum 1987; 26:424-429.

530. El-Khoury G, Ehara S, Weinstein JW et al. Epidural steroid injection: A procedure ideally performed with fluoroscopic control. Radiology 1988; 168:554-557.

531. Fredman B, Nun MB, Zohar E et al. Epidural steroids for treating "failed back surgery syndrome": Is fluoroscopy really necessary? Anesth Analg1999;88:367372.

532. Manchikanti L, Bakhit CE, Pakanati RR et al. Fluoroscopy is medically necessary for the performance of epidural steroids. Anesth Analg 1999; 89:1326-1327.

533. Stitz MY, Sommer HM. Accuracy of blind versus fluoroscopically guided caudal epidural injection. Spine 1999; 24:1371-1376.

534. Mehta M, Salmon N. Extradural block. Confirmation 
of the injection site by X-ray monitoring. Anaesthesia 1985; 40:1009-1012.

535. Nishimura N, Khahara T, Kusakabe T. The spread of lidocaine and 1-131 solution in the epidural space. Anesthesiology 1959; 20:785-788.

536. Burn JM, Guyer PB, Langdon L. The spread of solutions injected into the epidural space: A study using epidurograms in patients with lumbosciatic syndrome. Br J Anaesth 1973; 45:338-345.

537. Hodgson PSA, Mack B, Kopacz D et al. Needle placement during lumbar epidural anesthesia deviates toward the non-dependent side (abstract). Reg Anesth 1996; 21:26.

538. Bromage RP, Benumof JL. Paraplegia following intracord injection during attempted epidural anesthesia under general anesthesia. Reg Anesth Pain Med 1998; 23:104-107.

539. Krane EJ, Dalens BJ, Murat I, et al. The safety of epidurals during general anesthesia. Reg Anesth Pain Med 1998; 23:433-438.

540. Hodges SD, Castleberg RL, Miller T et al. Cervical epidural steroid injection with intrinsic spinal cord damage. Two case reports. Spine 1998; 23:2137-2142.

541. Derby R. Cervical epidural steroid injection with intrinsic spinal cord damage. Point of view. Spine 1998; 23:2141-2142.

542. Manchikanti L. Epidural steroid injections and intrinsic spinal cord damage. Spine 1999;24:1170-1171.

543. Apathy, A, Penczner G, Licker E et al. Caudal epidural injection in the management of lumbosacral nerve pain syndromes. Orv Hetil 1999; 140:1055-1058.

544. Breivik H, Hesla PE, Molnar I et al. Treatment of chronic low back pain and sciatica. Comparison of caudal epidural injections of bupivacaine and methylprednisolone with bupivacaine followed by saline. In Bonica JJ, Albe-Fesard D (eds). Advances in pain research and therapy. Raven Press, New York, 1976, Vol. 1, pp 927-932.

545. Bush K, Hillier S. A controlled study of caudal epidural injections of triamcinolone plus procaine for the management of intractable sciatica. Spine 1991; 16:572-575.

546. Matthews JA, Mills SB, Jenkins VM et al. Back pain and sciatica: Controlled trials of manipulation, traction, sclerosant and epidural injections. Brit $J$ Rheumatol 1987; 26:416-423.

547. Beliveau P. A comparison between epidural anesthesia with and without corticosteroids in the treatment of sciatica. Rheum Phys Med 1971; 11:40-43.

548. Czarski Z. Leczenie rwy kulszowej wstrzykiwaniem hydrokortyzonu inowokainy do rozworu kryzowego. Przeglad Kekarski 1965; 21:511-513.

549. Yates DW. A comparison of the types of epidural injection commonly used in the treatment of low back pain and sciatica. Rheum Rehab 1978; 17:181-186.

550. Daly P. Caudal epidural anesthesia in lumbosciatic pain. Anesthesia 1970; 25:346-348.
551. Ombregt L, Ter Veer HJ. Treatment of the lumbar spine. In Ombregt L, Bisschop P, Ter Veer HJ et al (eds). A System of Orthopaedic Medicine. WB Saunders, London, 1995, pp 633-688.

552. Ciocon JO, Galindo-Clocon D, Amarnath L et al. Caudal epidural blocks for elderly patients with lumbar canal stenosis. J Am Geriatr Soc 1994; 42:593596.

553. Manchikanti L, Pakanati RR, Pampati V. Comparison of three routes of epidural steroid injections in low back pain. Pain Digest 1999; 9:277-285.

554. Beyer W. Das zervikale and lumbale Bandscheibensyndrom und seind Behandlung mit NovocainPrednisolon-Injectionen an die Nervenwurzeln. Munch Med Wschr 1960; 102:1164-1165.

555. Lindhal O, Rexed B. Histologic changes in spinal nerve roots of operated cases of sciatica. Acta Orthop Scand 1951; 20:215-225.

556. Mount HTR. Epidural injection of hydrocortisone for the management of the acute lumbar disc protrusion. In Morley TP (ed). Current Controversies in Neurosurgery. WB Saunders, Philadelphia, 1976, pp 67-72.

557. Sharma RK. Indications, technique and results of caudal epidural injection for lumbar disc retropulsion. Postgrad Med J 1977; 53:1-6.

558. Gordon J. Caudal extradural injection for the treatment of low back pain. Anaesthesia 1980;35:515516.

559. Revel M, Auleley GR, Alaoui S et al. Forceful epidural injections for the treatment of lumbosciatic pain with post-operative lumbar spinal fibrosis. Rev Rhum Engl Ed 1996; 63:270-277.

560. Cuckler JM, Bernini PA, Wiesel SW et al. The use of epidural steroid in the treatment of radicular pain. $J$ Bone Joint Surg 1985; 67:63-66.

561. Dilke TFW, Burry HC, Grahame R. Extradural corticosteroid injection in the management of lumbar nerve root compression. Br Med J 1973; 2:635-637.

562. Helliwell M, Robertson JC, Ellia RM. Outpatient treatment of low back pain and sciatica by a single extradural corticosteroid injection. Br J Clin Pract 1985; 39:228-231.

563. Klenerman L, Greenwood R, Davenport HT et al. Lumbar epidural injections in the treatment of sciatica. Br J Rheumatol 1984; 23:35-38.

564. Ridley MG, Kingsley GH, Gibson T et al. Outpatient lumbar epidural corticosteroid injection in the management of sciatica. Br J Rheumatol 1988; 27:10031007.

565. Rocco AG, Frank E, Kaul AF et al. Epidural steroids, epidural morphine and epidural steroids combined with morphine in the treatment of post-laminectomy syndrome. Pain 1989; 36:297-303.

566. Serrao JM, Marks RL, Morley SJ et al. Intrathecal midazolam for the treatment of chronic mechanical low back pain: A controlled comparison with epidural steroid in a pilot study. Pain 1992;48:5-12. 
567. Snoek W, Weber H, Jorgensen B. Double-blind evaluation of extradural methylprednisolone for herniated lumbar disc. Acta Orthop Scand 1977; 48:635-641.

568. Rogers P, Nash T, Schiller D et al. Epidural steroids for sciatica. The Pain Clinic 1992; 5:67-72.

569. Castagnera L, Maurette P, Pointillart V et al. Longterm results of cervical epidural steroid injection with and without morphine in chronic cervical radicular pain. Pain 1994; 58:239-243.

570. Stav A, Ovadia L, Sternberg A et al. Cervical epidural steroid injection for cervicobrachialgia. Acta Anaesthesiol Scand 1993; 37:562-566.

571. Bush K, Hillier S. Outcome of cervical radiculopathy treated with periradicular/epidural corticosteroid injections: A prospective study with independent clinical review. Eur Spine J 1996; 5:319-325.

572. Brown FW. Management of discogenic pain using epidural and intrathecal steroids. Clin Orthop 1977; 129:72-78.

573. Warr AC, Wilkinson JA, Burn JMB et al. Chronic lumbosciatica syndrome treated by epidural injection and manipulation. Practitioner 1977; 209:53-59.

574. Hickey RF. Outpatient epidural steroid injections for low back pain and lumbosacral radiculopathy. NZMed $J$ 1987; 100:54-59.

575. Heyse-Moore GH. A rational approach to the use of epidural medication in the treatment of sciatic pain. Acta Orthop Scand 1978; 49:366-370.

576. Harley C. Extradural corticosteroid infiltration. A follow-up study of 50 cases. Ann Phy Med 1966; 9:2228.

577. Sharma S, Stedman R. Epidural steroids. A retrospective analysis of the efficacy of high and low dose therapy. Anesthesiology 1998; 3A:A1135.

578. Rosen CD, Kahanovitz N, Berstein R et al. A retrospective analysis of the efficacy of epidural steroid injections. Clin Orthop 1988;228:270-272.

579. Jamison RN, VadeBoncouer T, Ferrante FM. Low back pain patients unresponsive to an epidural steroid injection: Identifying predictive factors. Clin J Pain 1991; 7:311-317.

580. Fukusaki M, Kobayashi I, Hara T et al. Symptoms of spinal stenosis do not improve after epidural steroid injection. Clin J Pain 1998; 14:148-151.

581. Coomes EN. A comparison between epidural anesthesia and bedrest in sciatica. Brit Med J 1961;1:2024.

582. Catchlove RFH, Braha R. The use of cervical epidural nerve blocks in the management of chronic head and neck pain. Can Anaesth Soc J 1984; 31:188-191.

583. Berman AT, Garbarinbo JL, Fisher SM et al. The effects of epidural injection of local anesthetics and corticosteroids in patients with lumbosciatic pain. Clin Orthop 1984; 188:144-151.

584. Purkis IE. Cervical epidural steroids. Pain Clinic 1986; 1:3-7.
585. Rowlingson JC, Kirschenbaum LP. Epidural analgesic techniques in the management of cervical pain. Anesth Analg 1986; 65:938-942.

586. Warfield CA, Biber MP, Crews DA et al. Epidural steroid injection as a treatment for cervical radiculitis. Clin J Pain 1988; 4:201-204.

587. Cicala RS, Thoni K, Angel JJ. Long-term results of cervical epidural steroid injections. Clin J Pain 1989; 5:143-145.

588. Pawl RP, Anderson W, Shulman M. Effect of epidural steroids in the cervical and lumbar region on surgical intervention for discogenic spondylosis. In Fields HL (ed). Advances in Pain Research and Therapy.Vol.9; Raven Press, New York, 1985, pp 791-798.

589. Forrest JB. Management of chronic dorsal root pain with epidural steroids. Can Anesth SocJ 1978;25:218. Perkins HM, Hanlon PR. Epidural injection of local anesthetic and steroids for pain secondary to herpes zoster. Arch Surg 1978; 113:253.

591. Shulman M. Treatment of neck pain with cervical epidural steroid injection. Reg Anesth 1986; 11:92-94.

592. Zappala G. Iniezione peridurale segmentaria di Hydrocortone nella sindrome dolorosa da ernia discale. Policlinico - Sez Prat 1955; 62:1229-1231.

593. Yamazaka N. Interspinal injection of hydrocortisone or prednisolone in the treatment of intervertebral disc herniation. J Jpn Orthop Assoc 1959; 33:689.

594. Barry PJC, Kendall P. Corticosteroid infiltration of the extradural space. Ann Phys Med 1962; 6:267-273.

595. Hartman JT, Winnie AP, Ramaurthy S et al. Intradural and extradural corticosteroids for sciatica pain. Orthop Rev 1974; 3:21-24.

596. D'Hoogue R, Compere A, Gribmont B et al. Peridural injection of corticosteroids in the treatment of the low back pain/sciatica syndrome. Acta Orthop Belg 1976; 42:157-165.

597. Bullard JR, Houghton FM. Epidural treatment of acute herniated nucleus pulposus. Anesth Analg 1977; 56:862-863.

598. Jackson DW, Rettig A, Wiltse LL. Epidural cortisone injection in the young athletic adult. Am JSports Med 1980; 8:239-243.

599. Andersen KH, Mosdal C. Epidural application of corticosteroids in low-back pain and sciatica. Acta Neurochir 1987; 87:52-53.

600. Gupta AK, Singh R, Shukla RK et al. Role of epidural medication in the treatment of resistant low back pain. J Indian Med Assoc 1996; 94:224-226, 233.

601. Mam MK. Results of epidural injection of local anaesthetic and corticosteroid in patients with lumbosciatic pain. J Indian Med Assoc 1995; 93:17$18,24$.

602. Okuda Y, Suzuki K, Kitajima T et al. Lumbar epidural block for "painful legs and moving toes" syndrome: A report of three cases. Pain 1998; 78:145-147.

603. Reale C, Turkiewicz AM, Reale CA et al. Epidural 
steroids as a pharmacological approach. Clin Exp Rheumatol 2000; 18:S65-66.

604. Forrest JB. The response to epidural steroid injections in chronic dorsal root pain. Can Anaesth SocJ 1980; 27:40-46.

605. Rivest C, Katz JN, Ferrante FM et al. Effects of epidural steroid injection on pain due to lumbar spinal stenosis or herniated disks: A prospective study. Arthritis Care Res 1998; 11:291-297.

606. Bowman SJ, Wedderburn L, Whaley A et al. Outcome assessment after epidural corticosteroid injection for low back pain and sciatica. Spine 1993; 18:1345-1350.

607. Jurmand SH. Cortiotherapie peridurale des lombalgies et des sciatiques d'origine discale.Concours Medicale 1972; 94:5061-5070.

608. Ito R. The treatment of low back pain and sciatica with epidural corticosteroids injection and its pathophysiologic basis. JJpn Orthop Assoc 1971;45:769777.

609. Schmid G, Vetter S, Gottmann D et al. CT-guided epidural/perineural injections in painful disorders of the lumbar spine: Short- and extended-term results. Cardiovasc Intervent Radiol 1999; 22:493-498.

610. Fredman B, Zohar E, Ben Nun BM, Iraqi R et al. The effect of repeated epidural sympathetic nerve block on "failed back surgery syndrome" associated chronic low back pain. J Clin Anesth 1999; 11:46-51.

611. Katsaros GA, Handjis GP, Anastasiades J. Forty-eight single-shot epidural injections on the same patient. Anaesthesia 1990; 45:381-382.

612. Klein RG, Vaccaro AR, Cwik J et al. Efficacy of cervical epidural steroids in the treatment of cervical spine disorders. Am J Anesthesiol2000; (9):547-552.

613. Biella A, Cocognini P. L'acetato di idrocortisone nel trattamento della sindrome sciatalgica. Minerva Med 1954; 1:1863-1865.

614. Canale L. Il desametazone per via eidurale sacrale nelle lombosciataligie. Gaz Med Ital 1963; 122:210213.

615. Cappio M. Il trattamento idrocortisonico per via epidurale sacrale delle lombosciatalgie. Reumatismo 1957; 9:60-70.

616. Cappio M, Fragasso V. Osservazioni sull'uso dell'idroscortisone per via eidurale ed endorachidea nelle lombosciatalgie. Riforma Med 1955;22:605-607.

617. Cappio M, Fragasso V. Il prednisone per via epidurale sacrale nelle lombosciatalgie. Reumatismo 1957; 5:295-298.

618. Fragasso V. Il prednisolone idrosolubile per via epidurale sacrale nelle lombosciatalgie. Gaz Med Ital 1959; 118:358-360.

619. Gerest MF. Le traitement de la nevralgie sciatique par les injections epidurales d'hydrocortisone. JMed Lyon 1958; 261-264.

620. Gilly R. essai de traitement de 50 cas de sciatiques et de radiculalgies lombaires par le Clestene chronodose en infiltrations paradiculaire. Marseille Medicale1970; 107:341-345.

621. Renier JC. L'infiltration epidurale par le premier trou sacre posterieur. Revue du Rhumatisme et des Maladies Osteo-articulaire 1959; 26:526-532.

622. Riew KD, Yin Y, Gilula L, et al. Can nerve root injections obviate the need for operative treatment of lumbar radicular pain? A prospective, randomized, controlled, double-blind study. Proceedings of North American Spine Society, 14th Annual Meeting, Chicago. 1999, pp 94-95.

623. Shah R, Vad V, Lutz G et al. Transforaminal epidural injections in lumbar radiculopathy; prospective randomized trial. Proceedings of the North American Spine Society, $15^{\text {th }}$ Annual Meeting, New Orleans, 2000, pp 44-45.

624. Kraemer J, Ludwig J, Bickert U et al. Lumbar epidural perineural injection: A new technique. Eur Spine $J$ 1997; 6:357-361.

625. Lutz GE, Vad VB, Wisneski RJ. Fluoroscopic transforaminal lumbar epidural steroids: An outcome study. Arch Phys Med Rehabil 1998; 79:1362-1366.

626. Weiner BK, Fraser RD. Foraminal injection for lateral lumbar disc herniation. J Bone Joint Surg 1997; 79-B:804-807.

627. Devulder J. Transforaminal nerve root sleeve injection with corticosteroids, hyaluronidase, and local anesthetic in the failed back surgery syndrome. J Spinal Disord 1998; 11:151-154.

628. Slipman CW, Lipetz JS, Jackson HB et al. Therapeutic selective nerve root block in the nonsurgical treatment of atraumatic cervical spondylotic radicular pain: A retrospective analysis with independent clinical review. Arch Phys Med Rehabil 2000; 81:741-746.

629. Furman MB, O’Brien EM, Zgleszewski TM. Incidence of intravascular penetration in transforaminal lumbosacral epidural steroid injections. Spine 2000; 25:2628-2632.

630. Derby R, Kine G, Saal JA et al. Precision percutaneous blocking procedures for localizing spinal pain. Part 2: The lumbar neuraxial compartment. Pain Digest 1993; 3:175-188.

631. Kikuchi S. Anatomical and experimental studies of nerve root infiltration. JJPN Orthop Assoc 1982; 56:27-36.

632. Manchikanti L, Bakhit CE. Percutaneous lysis of epidural adhesions. Pain Physician 2000; 3:46-64.

633. Lou L, Racz GB, Heavner JE. Percutaneous epidural neuroplasty. In Waldman SD (ed).Interventional Pain Management. SecondEdition, WBSaunders Company, Philadelphia, 2000, pp 434-445.

634. Anderson SR, Racz GB, Heavner J. Evolution of epidural lysis of adhesions. Pain Physician 2000; 3:262270.

635. Lewandowski EM. The efficacy of solutions used in 
caudal neuroplasty. Pain Digest 1997; 7:323-330.

636. Racz GB, Holubec JT. Lysis of adhesions in the epidural space. In Racz GB (ed). Techniques of Neurolysis . Kluwer Academic Publishers, Boston, 1989, pp 5772.

637. Cooper R, Freemont A, Hoyland J et al. Herniated intervertebral disc-associated periradicular fibrosis and vascular abnormalities occur without inflammatory cell infiltration. Spine 1995; 20:591-598.

638. Cook SD, Prewett AB, Dalton JE et al. Reduction in perineural scar formation after laminectomy with Polyactive ${ }^{\circledR}$ membrane sheets. Spine 1994; 19:18151825.

639. Racz GB, Heavner JE, Diede JH. Lysis of epidural adhesions utilizing the epidural approach. In: Waldman $\mathrm{SD}$, Winnie AP (eds). Interventional Pain Management. WB Saunders, Philadelphia, 1996, pp 339-351.

640. Hatten HP Jr. Lumbar epidurography with metrizamide. Radiology 1980; 137:129-136.

641. Roberson GH, Hatten HP Jr, Hesselink JH. Epidurography. Selective catheter technique and review of 53 cases. Am J Radiol 1979; 132:787-793.

642. Devulder J, Lutgarde B, Castille F et al. Relevance of epidurography and epidural adhesiolysis in chronic failed back surgery patients. Clin J Pain 1995;11:147150.

643. Racz GB, Sabonghy M, Gintautas J et al. Intractable pain therapy using a new epidural catheter. JAMA 1982; 248:579-581.

644. Racz GB, Haynsworth RF, Lipton S. Experiences with an improved epidural catheter. Pain Clinic 1986;1:2127.

645. Racz GB, Heavner JE, Sigleton W et al. Hypertonic saline and corticosteroid injected epidurally for pain control. In: Racz GB (ed). Techniques of neurolysis . Boston, Kluwer Academic Publishers, 1989, pp 7394.

646. Manchikanti L. Pakanati RR, Bakhit CE et al. Role of adhesiolysis and hypertonic saline neurolysis in management of low back pain. Evaluation of Modification of Racz Protocol. Pain Digest, 1999;9:91-96.

647. Racz GB, Heavner JE, Raj PP. Percutaneous epidural neuroplasty. Prospective one-year follow up. Pain Digest 1999; 9:97-102.

648. Heavner JE, Racz GB, Raj P. Percutaneous epidural neuroplasty. Prospective evaluation of $0.9 \% \mathrm{NaCl}$ versus $10 \% \mathrm{NaCl}$ with or without hyaluronidase. $R e g$ Anesth Pain Med 1999; 24:202-207.

649. Manchikanti L, Pakanati RR, Bakhit CE, et al. Nonendoscopic and endoscopic adhesiolysis in post lumbar laminectomy syndrome. A one-year outcome study and cost effectiveness analysis. Pain Physician 1999; 2:52-58.

650. Arthur J, Racz G, Heinnrich R et al. Epidural space. Identification of filling defects in lysis of adhesions in the treatment of chronic painful conditions. In: $A b$ stracts, $7^{\text {th }}$ World Congress of Pain. Paris, IASP Pub- lications, 1993, pp 557.

651. Racz GB, Heavner JE. Relevance of epidurography: In response to article by Drs. Devulder et al. Clin J Pain 1995; 11:151-156.

652. Reed KL, Will K. Relevance of epidurography: In response to article by Drs. Devulder et al. Clin J Pain 1995; 11:151-156.

653. Annertz M, Jönsson B, Strömqvist B et al. No relationship between epidural fibrosis and sciatica in the lumbar postdiscectomy syndrome. Spine 1995;20:449453.

654. Burman MS. Myeloscopy or the direct visualization of the spinal cord. J Bone Joint Surg 1931; 13:695696.

655. Blomberg R. A method for spinal canal endoscopy and spinaloscopy: Presentation of preliminary results. Acta Anesth Scand 1985; 21:113-116.

656. Saberski LR, Kitahata L. Review of the clinical basis and protocol for epidural endoscopy. Connecticut Med 1995; 50:71-73.

657. Heavner JE, Chokhavatia S, Kizelshteyn G. Percutaneous evaluation of the epidural and subarachnoid space with the flexible fiberscope. Reg Anesth 1991; 15S1:85.

658. Saberski LR, Kitahata LM. Direct visualization of the lumbosacral epidural space through the sacral hiatus. Anesth Analg 1995; 80:839-840.

659. Saberski LR, Brull S. Fiberoptic visualization of the spinal cord. A historical review and report of current methods. Yale Biol Med 1995; 68:7-16.

660. Saberski LR. Spinal endoscopy: current concepts. In Waldman SD (ed). Interventional Pain Management. Second Edition, WB Saunders Company, Philadelphia, 2000, pp 143-161.

661. Manchikanti L, Pakanati RR, Pampati V. The value and safety of epidural endoscopic adhesiolysis. $A m J$ Anesthesiol 2000; 27:275-279.

662. Jalali S. Epidural endoscopic adhesiolysis: A valuable technique in treating refractory low back and extremity pain. Am J Anesthesiol 2000;27:261-264.

663. Saberski LR. A retrospective analysis of spinal canal endoscopy and laminectomy outcomes data. A pilot study. Pain Physician 2000; 3:193-196.

664. Saal JS, Saal JA. Management of chronic discogenic low back pain with a thermal intradiscal catheter: A preliminary study. Spine 2000;25:382-388.

665. Van Kleef M, Barendse GA. Percutaneous intradiscal radiofrequency thermocoagulation in chronic non-specific low back pain. Pain Clinic 1996; 3:259-268.

666. Derby R, Eek B, Chen Y et al. Intradiscal electrothermal annuloplasty (IDET): A novel approach for treating chronic discogenic back pain. Neuromodulation, 2000; 3(2):82-88.

667. Saal JA, Saal JS. Intradiscal electrothermal treatment for chronic discogenic low back pain. Spine 2000; 25:2622-2627.

668. Singh V. Intradiscal electrothermal therapy: A pre- 
liminary report. Pain Physician 2000; 3:367-373.

669. Karasek M, Bogduk N. Twelve-month follow-up of a controlled trial of intradiscal thermal annuloplasty for back pain due to internal disc disruption. Spine 2000; 25:2601-2607.

670. Wetzel FT, Andersson G, Pezola JH et al. Intradiscal electrothermal annuloplasty (IDET) to treat discogenic low back pain: Preliminary results of a multi-center. Cohort study: Proceedings of North American Spine Society, $15^{\text {th }}$ Annual Meeting, New Orleans, 2000, pp 195-197.

671. Derby R, O'Neill CW. The reported effects on referred leg pain post intradiscal electrothermal therapy. Proceedings of International Spinal Injection Society, San Francisco, September 8-10, 2000.

672. Liu B, Manos R, Criscitiello A et al. Clinical factors associated with favorable outcomes using intradiscal electrothermal modulation (IDET). Proceedings of Spine Across the Sea, Hawaii, July 23-27, 2000.

673. Maurer P, Schlemback D, Brown M. Lumbar intradiscal electrothermal annuloplasty (IDEA) for discogenic low back pain. ProceedingsofInternational Intradiscal Therapy Society Annual Meeting, Williamsburg, June 8-10, 2000.

674. Saal JA, Saal JS. Intradiscal electrothermal treatment (IDET) for chronic discogenic low back pain with two year follow-up. Proceedings of North American Spine Society, $15^{\text {th }}$ Annual Meeting, New Orleans, 2000, pp 5-7.

675. Lee J, Lutz GE, Campbell D et al. Stability of the spine after intradiscal electrothermal therapy. Proceedings of the International Spinal Injection Society, $5^{\text {th }}$ Annual Scientific Meeting,Las Vegas, August 13-15, 1999.

676. Manchikanti L. The role of radiofrequency in the management of complex regional pain syndrome. Cur Rev Pain 2000; 4:437-444.

677. Elias M. Cervical sympathetic and stellate ganglion blocks. Pain Physician 2000; 3:294-304.

678. Raja S. Reflex sympathetic dystrophy: Pathophysiological basis for therapy. Pain Digest 1992; 2:274280.

679. Stanton-Hicks M, Raj P, Racz G. Use of regional anesthetics for diagnosis of reflex sympathetic dystrophy and sympathetically maintained pain; A critical evaluation. In Jänig W, Stanton-Hicks M (eds). Reflex Sympathetic Dystrophy: A Reappraisal. Progress in Pain Management and Research. IASP Press; Seattle, 1996, pp 217-237.

680. Wilkinson H. Percutaneous radiofrequency, upper thoracic sympathectomy. New Technique. Neurosurgery 1984; 15:811-814.

681. Wilkinson $\mathrm{H}$. Neurosurgical procedures of the sympathetic nervous system. Pain Clinic 1995; 1:43-50.

682. Elias M. The anterior approach for thoracic sympathetic ganglion block using a curved needle. Pain
Clinic 2000; 12:17-24.

683. Malmqvist EL, Bengtsson M, Sorenson J. Efficacy of stellate ganglion block: A clinical study with bupivacaine. Reg Anesth 1992; 17:340-347.

684. Geurts JW, Stolker RJ. Percutaneous radiofrequency lesion of the stellate ganglion in the treatment of pain in upper extremity reflex sympathetic dystrophy. Pain Clinic 1993; 6:17-25.

685. Eisenberg E, Carr DB, Chalmers TC. Neurolytic celiac plexus block for treatment of cancer pain: A metaanalysis. Anesth Analg 1995; 80:290-295.

686. Ischia S, Ischia A, Polati E et al. Three posterior percutaneous celiac plexus block techniques. Anesthesiology 1992; 76:534-540.

687. Mercadante $S$. Celiac plexus block versus analgesics in pancreatic cancer pain. Pain 1993; 52:187-192.

688. Ventafridda GV, Caraceni AT, Sbanotto AM et al. Pain treatment in cancer of the pancreas. Eur JSurg Oncol 1990; 16:1-6.

689. Hegedus V. Relief of pancreatic pain by radiographyguided block. Am J Radiol 1979; 133:1101-1103.

690. Bridenbaugh LD, Moore DC, Campbell DD. Management of upper abdominal cancer pain. JAMA 1964; 190:877-880.

691. Krames E. Spinal cord stimulation: Indications, mechanism of action, and efficacy. Cur Rev Pain 1999; 3:419426.

692. North RB, Ewend MG, Lawton MT et al. Spinal cord stimulation for chronic, intractable pain: Superiority of "multi-channel" devices. Pain 1991; 44:119-130.

693. Barolat G, Schwartzman R, Woo R. Epidural spinal cord stimulation in the management of reflex sympathetic dystrophy. Stereotact Funct Neurosurg 1989; 53:29-30.

694. Oakley JC, Weiner RL. Spinal cord stimulation for complex regional pain syndrome: A prospective study of 19 patients at two centers. Neuromodulation 1999; 2:47-50.

695. Kemler MA, Barendse G, van Kleef M et al. Spinal cord stimulation in patients with chronic reflex sympathetic dystrophy. NEngl JMed 2000; 343:618-624. 696. Schwartzman RJ. New treatments for reflex sympathetic dystrophy. N Engl J Med 2000; 343:654-656.

697. Burcheil KJ, Anderson VC, Brown FD et al. Prospective, multicenter study of spinal cord stimulation for relief of chronic back and extremity pain. Spine 1996; 21:2786-2794.

698. Vulink N, Overgaauw DM, Jessurun G et al. The effects of spinal cord stimulation on quality of life in patients with therapeutically chronic refractory angina pectoris. Neuromodulation 1999; 2:33-40.

699. Claeys L. Spinal cord stimulation and chronic critical limb ischemia. Neuromodulation 1999; 2:33-40.

700. Augustinsson LE, Holm J, Carl A et al. Epidural electrical stimulation in severe limb ischemia: Evidences of pain relief, increased blood flow and a possible limb 
saving effect. Ann Surg 1985;202:104-111.

701. Jacobs MJ, Jorning PJ, Joshi SR et al. Epidural spinal cord electrical stimulation improves microvascular blood flow in severe limb ischaemia. Ann Surg 1988; 207:179-183.

702. Shealy CN, Mortimer JT, Reswick JB. Electrical inhibition of pain by stimulation of the dorsal columns: Preliminary clinical report. Anesth Analg 1967; 46:489-491.

703. Melzack R, Wall PD. Pain mechanisms: A new theory. Science 1965; 150:971-978.

704. Wetzel FT, Hassenbusch S, Oakley JC et al. Treatment of chronic pain in failed back surgery patients with spinal cord stimulation: A review of current literature and proposal for future investigation. Neuromodulation 2000; 3:59-74.

705. Devulder J, Laat MD, Bastelaere MV. Spinal cord stimulation: A valuable treatment for chronic failed back surgery patients. JPain Symptom Manage;1997; 13:296-301.

706. Neilson KD, Adams JE, Hosobuchi Y. Experience with dorsal column stimulation for relief of chronic intractable pain: 1968-1973. Surg Neurol 1975; 4:148-152.

707. Shelden CH, Paul F, Jacques DB et al. Electrical stimulation of the nervous system. Surg Neurol 1975;4:127132.

708. Richardson RR, Siqueria EB, Cerullo LJ. Spinal epidural neurostimulation for treatment of acute and chronic intractable pain: Initial and long term results. Neurosurgery 1979; 5:344-348.

709. Kumar K, Nath R, Wyant GM. Treatment of chronic pain by epidural spinal cord stimulation: A 10-year experience. J Neurosurg 1991;75:402-407.

710. North RB, Ewend MG, Lawton MT et al. Failed back surgery syndrome: 5-year follow-up after spinal cord stimulatorimplantation. Neurosurgery 1991;28:692699.

711. North RB, Kidd DH, Lee MS et al. A prospective, randomized study of spinal cord stimulation versus reoperation for failed back surgery syndrome: Initial results. Sterotact Funct Neurosurg 1994; 62:267-272.

712. De La Porte C, Van de Kelft E. Spinal cord stimulation in failed back surgery syndrome. Pain 1993; 52:55-61.

713. North RB, Kidd DH, Zahurak M et al. Spinal cord stimulation for chronic intractable pain: experience over two decades. Neurosurgery 1993; 32:384-395.

714. Tesfaye S, Watt J, Benbow SJ et al. Electrical spinalcord stimulation for painful diabetic peripheral neuropathy. Lancet 1996; 348; 1698-1701.

715. Willis KD, Doleys DM. The effects of long-term intraspinal infusion therapy with noncancer pain patients: Evaluation of patient, significant - other, and clinic staff appraisals. Neuromodulation 1999; 2:241-253.

716. Portenoy RK. Opioid therapy for chronic non-malignant pain: Current status. In Nin HI, Fields DS,
Liebeskind JC (eds). Pharmacological Approaches to the Treatment of Chronic Pain: Concepts and Critical Issues. IASP Press; Seattle, 1994, pp 247-288.

717. Portenoy RK, Foley KM. Chronic use of opioid analgesics in non-malignant pain. Report of 38 cases. Pain 1986; 25:171-186.

718. Pappagallo M, Raja SN, Haytheornwaite JA et al. Oral opioids in the management of postherpetic neuralgia: A prospective survey. Analgesia 1994; 1:51-55.

719. Krames ES, Lanning RM. Intrathecal infusional analgesia for non-malignant pain: analgesic efficacy of intrathecal opioids with and without bupivacaine. $J$ Pain Symptom Manage 1993; 8:539-548.

720. Maron J, Loeser JD. Spinal opioid infusion in treatment of chronic pain of non-malignant origin. Clin J Pain 1996; 12:174-179.

721. Krames ES, Schuchard M. Implantable intraspinal infusion analgesia: Management guidelines. PainRev 1995; 2:243-267.

722. Schuchard M, Lanning R, North R et al. Neurologic sequelae of intraspinal drug delivery systems: Results of a survey of American implanters of implantable drug delivery systems. Neuromodulation 1998;1:137-148. Doleys DM, Coleton M, Tutak U. Use of intraspinal infusion therapy with non-cancer pain patients. Follow up and comparison of worker's compensation versus non-worker's compensation patients. Neuromodulation 1998; 1:149-159.

724. Hassenbusch SJ, Stanton-Hicks M, Covington EC et al. Long-term intraspinal infusion of opiates in the treatment of neuropathic pain. JPain Symptom Manage 1995 ; 10:527-543.

725. Winkelmüller M, Winkelmüller F. Long-term effects of continuous intrathecal opioid treatment in chronic pain of non-malignant etiology. JNeurosurg 1996; 85:458-467.

726. Paice JA, Penn RD, Shott S. Intraspinal morphine for chronic non-cancer pain. A retrospective multi-center study. J Pain Symptom Manage 1996;11:71-80.

727. Tutak U, Doleys DM. Intrathecal infusion systems for the treatment of chronic low back and leg pain of noncancer origin. South Med J1996; 89:295-300.

728. Hilten BJV, Willem-Johan T, Beek VD et al. Intrathecal baclofen for the treatment of dystonia in patients with reflex sympathetic dystrophy. $N$ Engl J Med2000; 343:625-630.

729. Avellino AM, Loeser JD. Intrathecal baclofen for the treatment of intractable spasticity of spine or brain etiology. Neuromodulation 2000; 3:75-81.

730. Waldman SD. Complications of cervical epidural nerve blocks with steroids: A prospective study of 790 consecutive blocks. Reg Anesth 1989; 14:149-151.

731. Katz JA, Lukin R, Bridenbaugh PO et al. Subdural intracranial air: An unusual cause of headache after epidural steroid injection. Anesthesiology 1991; 
74:615.

732. Mateo E, Lopez-Alarcon MD, Moliner S et al. Epidural and subarachnoid pneumocephalus after epidural technique. EurJ Anesethsiol 1999; 16:413-17.

733. Schmidt SD, Gibbons JJ. Post dural puncture headache after fluoroscopically guided lumbar paravertebral sympathetic block. Anesthesiology 1993; 78:198200.

734. Gladstone JC, Pennant JH. Spinal anaesthesia following facet joint injection. Anaesthesia 1987; 42:754756.

735. Marks R, Semple AJ. Spinal anaesthesia after facet joint injection. Anaesthesia 1988; 43:65-66.

736. Williams KN, Jackowski A, Evans PJ. Epidural haematoma requiring surgical decompression following repeated cervical epidural steroid injections for chronic pain. Pain 1990; 42:197-199.

737. Benzon HT, Wong HY, Siddiqui T et al. Caution in performing epidural injections in patients on several antiplatelet drugs. Anesthesiology 1999;91:15581559.

738. Horlocker TT, Wedel DJ, Offord KP. Does preoperative antiplatelet therapy increase the risk of hemorrhagic complications associated with regional anesthesia? Anesth Analg 1990;70:631-634

739. Pounder D, Elliott S. An awake patient may not detect spinal cord puncture. Anaesthesia 2000;55:194.

740. Sampath P, Rigamonti D. Spinal epidural abscess: A review of epidemiology, diagnosis, and treatment. $J$ Spinal Disord 1999; 12:89-93.

741. Shealy CN. Dangers of spinal injections without proper diagnosis. JAMA 1966; 197:1104-1106.

742. Waldman SD. Cervical epidural abscess after cervical epidural nerve block with steroids (Letter). Anesth Anal 1991; $72: 717$.

743. Mamourian AC, Dickman CA, Drayer BP et al. Spinal epidural abscess: Three cases following spinal epidural injection demonstrated with magnetic resonance imaging. Anesthesiology 1993; 78:204.

744. Knight JW, Cordingley JJ, Palazzo MGA. Epidural abscess following epidural steroid and local anaesthetic injection. Anaesthesia 1997; 52:576.

745. Strong, WE. Epidural abscess associated with epidural catheterization: A rare event? Report of two cases with markedly delayed presentation. Anesthesiology 1991; 74:943.

746. Bromage PR. Spinal extradural abscess: Pursuit of vigilance. BrJ Anaesth 1993; 70:471-473.

747. Dougherty JH, Fraser RAR. Complications following intraspinal injections of steroids. JNeurosurg 1978; 48:1023-1025.

748. Gutknecht DR. Chemical meningitis following epidural injections of corticosteroids (Letter). Am J Med 1987; 82:570.

749. Yap KB, Finaly IG. Epidural infection associated with epidural catheterization in a cancer patient with back pain: Case report. Palliat Med 1994; 8:251-253.

750. Kaul S, Meena AK, Sundaram C et al. Spinal extradural abscess following local steroid injection. Neurol India 2000; 48:181-183.

751. Elias M. Cervical epidural abscess following trigger point injection. JPain Symptom Manage 1994; 9:7172.

752. Wang LP, Haverberg J, Schmidt JF. Incidence of spinal epidural abscess after epidural analgesia. Anesthesiology 1999; 91:1928-1936.

753. Chan ST, Leung S. Spinal epidural abscess following steroid injection for sciatica: Case report. Spine 1989; 14:106-108.

754. Williamson JA. Inadvertent spinal subdural injection during attempted spinal epidural steroid therapy. Anaesth Intens Care 1990;18:406-408.

755. Sekel R. Epidural depo-medrol revisited. MedJAust 1984; 141:688.

756. Goucke CR, Graziotti P. Extradural abscess following local anaesthetic and steroid injection for chronic low back pain. Brit J Anesth 1990; 65:427-429.

757. Lindner A, Warmuth-Metz M, Becker G et al. Iatrogenic spinal epidural abscesses: Early diagnosis essential for good outcome. Eur J Med Res 1997; 2:201205.

758. O'Brien DP, Rawluk DJ. Iatrogenic mycobacterium infection after an epidural injection. Spine 1999; 24:1257-1259.

759. Schiller F, Shadle OW. Extrathecal and intrathecal suppuration. Arch Neurol 1962; 7:33-36.

760. Vogelsang H. Discitis intervertebralis cervicalis nach diskographie. Neurochirurgia 1973; 16:80-83.

761. Lubenow T, Keh-Wong E, Kristof Ket al. Inadvertent subdural injection: A complication of an epidural block. Anesth Analg 1988; 67:175-179.

762. Lehmann LJ, Pallares VS. Subdural injection of a local anesthetic with steroids: Complication of epidural anesthesia. South Med J1995; 88:467-469.

763. Siegfried RN. Development of complex regional pain syndrome after a cervical epidural steroid injection. Anesthesiology 1997; 86:1394-1396.

764. Thomson SJ, Lomax DM, Collett BJ. Chemical meningism after lumbar facet joint block with local anaesthetic and steroids. Anaesthesia 1991; 46:563-564.

765. Ling C, Atkinson PL, Munton CGF. Bilateral retinal hemorrhages following epidural injection. $\mathrm{Br} J$ Ophthalmol 1993; 77:316.

766. Knight CL, Burnell JC. Systemic side-effects of extradural steroids. Anaesthesia 1980;35:593-594.

767. Jacobs A., Pullan PT, Potter JM et al. Adrenal suppression following extradural steroids. Anaesthesia 1983; 38:953-956.

768. Mikhail GR, Sweet LC, Mellinger RC. Parenteral longacting corticosteroid effect on hypothalamic pituitary adrenal function. Ann Allergy 1973;31:337-343. 
769. Mikhail Gr, Livingood Cs, Mellinger RC et al. Effect of long-acting parenteral corticosteroids on adrenal function. Arch Dermatol 1969; 100:263-268.

770. Melby JC. Drug spotlight program: Systemic corticosteroid therapy; pharmacologic and endocrinologic considerations. Ann Intern Med 1974; 81:505-512.

771. Schimmer BP, Parker KL. Adrenocorticotropic hormone; adrenocortical steroids and their synthetic ana$\log$; inhibitors of the synthesis and actions of adrenocortical hormones. In Harman JG, Molinoff PB, Ruddon RW (eds).Goodman's \& Gilman's , ThePharmacological Basis of Therapeutics, Ninth Edition, McGraw-Hill, New York, 1996, pp 1459-1485.

772. McEvoy GK, Litvak K, Welsh OH et al. Adrenals. AHFS 99 drug information. American Society of Health-System Pharmacists, Bethesda, 1999, pp 26362662.

773. Boonen S, Van Distel G, Westhovens R et al. Steroid myopathy induced by epidural triamcinolone injection. Brit J Rheumatol 1995;34:385.

774. Sandberg DI, Lavyne MH. Symptomatic spinal epidural lipomatosis after local epidural corticosteroid injections: Case report. Neurosurgery 1999; 45:162165.

775. Manchikanti L, Pampati VS, Beyer C et al. The effect of neuraxial steroids on weight and bone mass density: A prospective evaluation. Pain Physician 2000; 3:357-366.

776. Aldrete JA, Zapata JC, Ghaly R. Arachnoiditis following epidural adhesiolysis with hypertonic saline report of two cases. Pain Digest 1996; 6:368-370.

777. Heavner JE. Comments on arachnoiditis following epidural adhesiolysis. Pain Digest 1997; 7:157.

778. Manchikanti L. Comments on arachnoiditis following epidural adhesiolysis. Pain Digest 1997; 7:157158.

779. Erdine S, Ozyalcin S. Comments on arachnoiditis following epidural adhesiolysis. Pain Digest 1997; 7:158159.

780. Kim RC, Porter RW, Choi BH et al. Myelopathy after intrathecal administration of hypertonic saline. $\mathrm{Neu}$ rosurgery 1988; 22:942-944.

781. Lucas JS, Ducker TB, Perot PL. Adverse reactions to intrathecal saline injections for control of pain. $J$ Neurosurg 1975; 42:57-561.

782. Hitchcock ER, Prandini MN. Hypertonic saline in management of intractable pain. Lancet 1973; 1:310312.

783. Manchikanti L, Bakhit CE. Removal of torn Racz catheter from lumbar epidural space. Reg Anesth 1997; 22:579-581.

784. Delaney TJ, Rowlingson JC, Carron Het al. Epidural steroid effects on nerves and meninges. Anesth Analg 1980; 58:610-614.

785. Cicala RS, Turner R, Moran E et al. Methylprednisolone acetate does not cause inflammatory changes in the epidural space. Anesthesiology 1990;72:556-558.
786. MacKinnon Se, Hudson AR, Gentilli R et al. Peripheral nerve injection injury with steroid agents. Plast Reconstr Surg 1982;69:482-489.

787. Chino N, Awad EA, Kottke FJ. Pathology of propylene glycol administered by perineural and intramuscular injection in rats. Arch Phys Med Rehab 1974; 55:33-38.

788. Benzon HT, Gissen AJ, Strichartz GR et al. The effect of polyethylene glycol on mammalian nerve impulses. Anesth Analg 1987;66:553-559.

789. Abram SE, Marsala M, Yaksh TL. Analgesic and neurotoxic effects of intrathecal corticosteroids in rats. Anesthesiology 1994; 81:1198-1205.

790. Latham JM, Fraser RD, Moore RJ et al. The pathologic effects of intrathecal betamethasone. Spine 1997; 22:1558-1562.

791. Hammer M, Meneese W. Principles and practice of radiofrequency neurolysis. Cur Rev Pain 1998;2:267278.

792. Saberski LR, Ahmad M, Fitzgerald J et al. Practice patterns in the use of epidural corticosteroid injections. A survey of the Connecticut State Society of Anesthesiologists. (Poster Exhibits). Anesthesiology 1998; 101.

793. Hopwood M. Outcomes assessment in pain management. In Abram SE (ed). Pain Management. Churchill-Livingston, Philadelphia, 1998, pp 14.114.11.

794. Piccirillo JF. Outcomes research and otolaryngology. Otolaryngol Head Neck Surg 1994; 111:764-769.

795. Ross Davies A, Doyle AT, Lansky D et al. Outcomes assessment in clinical settings: A consensus statement on principles and best practices in project management. Jt Comm J Qual Improvement 1994; 20(1):6-16.

796. The Foundation for Health Services Research. Health Outcomes Research Primer. The Foundation for Health Services Research, Washington, 1994.

797. Epstein RS, Sherwood LM. From outcomes research to disease management: A guide for the perplexed. Ann Intern Med 1996; 124:832-837.

798. Greenfield S, Kaplan SH, Silliman RA et al. The uses of outcomes research for medical effectiveness, quality of care, and reimbursement in Type II diabetes. Diabetes Care 1994; 17:32-39.

799. Lynn J, Virnig BA. Assessing the significance of treatment effects: Comments from the perspective of ethics. Med Care 1995; 33:AS292-AS298.

800. Wilson IB, Kaplan S. Clinical practice and patient health status: How are the two related? Med Care 1995; 33:AS209-AS214.

801. Fowler FJ. Using patient reports to evaluate medical outcomes. United States Congress, Office of Technology Assessment. Tools for Evaluating Health Technologies. Background Paper Five, BP-H-142. U.S. Government Printing Office, Washington, February, 1995.

802. Cicala RS, Wright H. Outpatient treatment of patients 
with chronic pain. Analysis of cost savings. Clin J Pain 1989; 5:223-226.

803. Goossens MEJB, Evers SMAA, Vlaeyen JWS et al. Principles of economic evaluation for interventions of chronic musculoskeletal pain. Eur J Pain 1999;3:343353.

804. Ferraz MB, Maetzel A, Bombardier C. A summary of economic evaluations published in the field of rheumatology and related disciplines. Arthr Rheum 1997; 40:1587-1593.

805. Goossens MEGJB, Evers SMA. Economic evaluation of back pain interventions. J Occup Rehab 1997; 7:1532.

806. Maetzel A, Ferraz, MB, Bombardier C. A review of cost-effectiveness analyses in rheumatology and related disciples. Current Opin Rheumatol 1998; 10:136-140.

807. Malter AD, Larwon EB, Urban N et al. Cost-effectiveness of lumbar discectomy for the treatment of herniated intervertebral disc. Spine 1996; 21:1048-1055.

808. Mueller-Schwefe G, Hassenbusch SJ, Reig E. Costeffectiveness of intrathecal therapy for pain. Neuromodulation 1999; 2:77-84.

809. Kuntz K, Snider R, Weinstein J et al. Cost-effectiveness of fusion with and without instrumentation for patients with degenerative spondylolisthesis and spinal stenosis. Spine 2000; 25:1132-1139.

810. Guo HR, Tanaka S, Halperin WE et al. Back pain prevalence in US industry and estimates of lost work days. Am J Public Health 1999; 89:1029-1035.

811. Lave JR, Frank RG, Schulberg HC et al. Cost effectiveness of treatments for major depression in primary care practice. Arch Gen Psychiatry 1998; 55:645-651.

812. Chang RW, Pellissier JM, Hazen GB. A cost-effectiveness analysis of total hip arthroplasty of osteoarthritis of the hip. JAMA 1996;275:858-865.

813. Wong JB, Sonnenberg FA, Salem DN et al. Myocardial revascularization for chronic stable angina: Analysis of the role of percutaneous transluminal coronary angioplasty based on data available in 1989. Ann Intern Med 1990; 113:852-871.

\section{APPENDIX I}

Laxmaiah Manchikanti, MD, Medical Director, Pain Management Center of Paducah, 2831 Lone Oak Road, Paducah, KY 42003, Email: drm@asipp.org.

Vijay Singh, MD, Medical Director, Pain Diagnostic Associates, 1601 Roosevelt Road, Niagara, WI 54151, Email: vsingh@netnet.net.
David Kloth, MD, Medical Director, Connecticut Pain Care, PC, 69 Sand Pit Road, Suite 204, Danbury, CT, 06810, Email: dkmd@ctpaincre.com.

Curtis W. Slipman, MD, Medical Director, Penn Spine Center, Rehabilitation Medicine, Assistant Professor, University of Pennsylvania Health System, 3400 Spruce Street, Great White, PA 19104,

Email: slipman@mail.med.upenn.edu.

Joseph F. Jasper, MD, Medical Director, Advanced Pain Medicine Physicians, 1628 South Mildred Street, Suite \#105, Tacoma, WA 98465, Email: apmedicine@ qwest.net.

Andrea M. Trescot, MD, Medical Director, Pain Center, 1895 Kingsley Ave. Suite 903, Orange Park, FL 32073, Email: amt57@aol.com.

Kenneth G. Varley, MD, Medical Director, Southern Pain Specialists, 7500 Hugh Daniel Drive, \#360, Birmingham, AL 35242, Email: gaynes@aol.com.

Sairam L. Atluri, MD, Medical Director, Eastern Cincinati Pain Management Associates, 8000 State Road, Suite 325, Cincinati, OH 45230, Email: atluri_ps@sprynet.com.

Carlos Giron, MD, Medical Director, Georgia Pain Institute, 797 Poplar Street, Macon, GA 31201, Email: cgiron@worldnet.att.net.

Mary Jo Curran, MD, Medical Director, Advanced Pain Treatment Centers, 676 N St. Clair, Suite 360, Chicago IL 60611, Email: mj@bepainfree.com.

Jose Rivera, MD, Interventional Pain Physician, Pain Management Center of Paducah, 2831 Lone oak Road, Paducah, KY 42003, Email: painmgmt@apex.net.

A Ghafoor Baha, MD, Interventional Pain Physician, Pain Management Center of Paducah, 2831 Lone oak Road, Paducah, KY 42003, phone (270) 554-8373, fax (270) 5548987 or Email: painmgmt@apex.net.

Cyrus E. Bakhit, MD, Medical Director, Pain Management Center of Roanoke, 1119 South Jefferson Street, Roanoke, VA 24016, Email: cbakhit@pmcr.org.

Merrill W. Reuter, MD, Medical Director, Lake Worth Medical Center, 7625 Lake Worth Road, Lake Worth, FL 33467, Email: aospine@doctor.com. 


\section{Alphabetical listing of references}

Abram SE, Marsala M, Yaksh TL. Analgesic and neurotoxic effects of intrathecal corticosteroids in rats. Anesthesiology 1994; 81:1198-1205.

Abram SE. Current guidelines in the use of epidural steroids in the United States of America. Pain Digest 1999; 9:233-234.

Abram SE. Treatment of lumbosacral radiculopathy with epidural steroids. Anesthesiology 1999; 91:1937-1941.

Airaksinen O, Herno A, Turunen V et al. Surgical outcome of 438 patients treated surgically for lumbar spine stenosis. Spine 1997; 22:2278-2282.

Aldrete JA, Zapata JC, Ghaly R. Arachnoiditis following epidural adhesiolysis with hypertonic saline report of two cases. Pain Digest 1996; 6:368-370.

Allen G, Galer B, Schwartz L. Epidemiology of complex regional pain syndrome: A retrospective chart review of 134 patients. Pain 1999; 80:539-544.

Altmaier EM, Lehmann T, Russell DW et al. The effectiveness of psychological interventions for the rehabilitation of low back pain: A randomized controlled trial evaluation. Pain 1992; 49:329-335.

American Geriatrics Society. The management of chronic pain in older persons: New guidelines from the American Geriatrics Society. J Am Geriatr Soc 1998; 46:128-150.

American Pain Society. Guideline for the management of acute and chronic pain in sickle-cell disease. American Pain Society, Glenview, 1999.

Andersen KH, Mosdal C. Epidural application of corticosteroids in low-back pain and sciatica. Acta Neurochir 1987; 87:5253.

Anderson GBJ, Svensson HO. The intensity of work recovery in low back pain. Spine 1983; 8:880-887.

Anderson SR, Racz GB, Heavner J. Evolution of epidural lysis of adhesions. Pain Physician 2000; 3:262-270.

Andersson HI, Ejlertsson E, Leden I et al. Characteristics of subjects with chronic pain, in relation to local and widespread pain report. Scand J Rheumatol 1996; 25:146-154.

Andersson HI, Ejlertsson G, Leden I et al. Chronic pain in a geographically defined general population: Studies of differences in age, gender, social class, and pain localization. Clin J Pain 1993; 9:174-182.

Annertz M, Jönsson B, Strömqvist B et al. No relationship between epidural fibrosis and sciatica in the lumbar postdiscectomy syndrome. Spine 1995; 20:449-453.

Apathy, A, Penczner G, Licker E et al. Caudal epidural injection in the management of lumbosacral nerve pain syndromes. Orv Hetil 1999; 140:1055-1058.

Aprill C, Dwyer A, Bogduk N. The prevalence of cervical zygapophyseal joint pain patterns II: A clinical evaluation. Spine 1990; 6:458-461.

Arthur J, Racz G, Heinnrich R et al. Epidural space. Identification of filling defects in lysis of adhesions in the treatment of chronic painful conditions. In: Abstracts, $7^{\text {th }}$ World Congress of Pain. Paris, IASP Publications, 1993, pp 557.
Asch SM, Sloss EM, Hogan C et al. Measuring underuse of necessary care among elderly Medicare beneficiaries using inpatient and outpatient claims. JAMA 2000; 284:2325-2333.

Attributes to guide the development of practice parameters. American Medical Association, Chicago, 1994.

Augustinsson LE, Holm J, Carl A et al. Epidural electrical stimulation in severe limb ischemia: Evidences of pain relief, increased blood flow and a possible limb saving effect. Ann Surg 1985; 202:104-111.

Avellino AM, Loeser JD. Intrathecal baclofen for the treatment of intractable spasticity of spine or brain etiology. Neuromodulation 2000; 3:75-81.

Badley EM, Tennant A. Changing profile of joint disorders with age. Findings from a postal survey of the population of Calderdale, West Yorkshire, United Kingdom. Ann Rheum Dis 1992; 51:366-371.

Banerjee T, Pittman HH. Facet rhizotomy. Another armamentarium for treatment of low backache. NC Med J 1976; 37:354360.

Barnsley L, Bogduk N. Medial branch blocks are specific for the diagnosis of cervical zygapophyseal joint pain. Reg Anesth 1993; 18:343-350.

Barnsley L, Lord S, Bogduk N. Comparative local anesthetic blocks in the diagnosis of cervical zygapophysial joints pain. Pain 1993; 55:99-106.

Barnsley L, Lord S, Bogduk N. Whiplash injury. Pain 1994; 58:238-307.

Barnsley L, Lord SM, Wallis BJ et al. Lack of effect of intraarticular corticosteroids for chronic pain in the cervical zygapophyseal joints. N Engl J Med 1994; 330:1047-1050.

Barnsley L, Lord SM, Wallis BJ et al. The prevalence of chronic cervical zygapophyseal joint pain after whiplash. Spine 1995; 20:20-26.

Barolat G, Schwartzman R, Woo R. Epidural spinal cord stimulation in the management of reflex sympathetic dystrophy. Stereotact Funct Neurosurg 1989; 53:29-30.

Barry PJC, Kendall P. Corticosteroid infiltration of the extradural space. Ann Phys Med 1962; 6:267-273.

Battista RN, Hodge MJ. Clinical practice guidelines: Between science and art. Can Med Assoc J 1993; 148:385.

Beliveau P. A comparison between epidural anesthesia with and without corticosteroids in the treatment of sciatica. Rheum Phys Med 1971; 11:40-43.

Benoist M, Ficat C, Baraf P, et al. Post operative lumbar epiduroarachnoiditis: Diagnostic and therapeutic aspects. Spine 1980; 5:432-436.

Benzon HT, Gissen AJ, Strichartz GR et al. The effect of polyethylene glycol on mammalian nerve impulses. Anesth Analg 1987; 66:553-559.

Benzon HT, Molly RE. Outcomes, efficacy, and compliances from management of low back pain. In Raj PP, Abrams BM, Benzon HT et al (eds), Practical management of pain. Third Edition, Mosby, Philadelphia 2000, pp 891-903. 
Benzon HT, Wong HY, Siddiqui T et al. Caution in performing epidural injections in patients on several antiplatelet drugs. Anesthesiology 1999; 91:1558-1559.

Benzon HT. Epidural steroid injections for low back pain and lumbosacral radiculography. Pain 1986; 24:277.

Benzon HT. Epidural steroid injections. Pain Digest 1992; 1:271-280.

Bergstrom G, Bjelle A, Sorensen LB et al. Prevalence of symptoms and signs of joint impairment at age 79. Scand J Rehab Med 1985; 17:173-182.

Bergstrom G, Bjelle A, Sundh V et al. Joint disorders at age 70, 75 , and 79 years. A cross-sectional comparison. BrJ Rheumatol 1986; 23:333-341.

Berman AT, Garbarinbo JL, Fisher SM et al. The effects of epidural injection of local anesthetics and corticosteroids in patients with lumbosciatic pain. Clin Orthop 1984; 188:144151.

Bernini PM, Simeone FA. Reflex sympathetic dystrophy associated with lumbar disc herniation. Spine 1981; 6:180-184.

Beyer W. Das zervikale and lumbale Bandscheiben-syndrom und seind Behandlung mit Novocain-Prednisolon-Injectionen an die Nervenwurzeln. Munch Med Wschr 1960; 102:11641165.

Biella A, Cocognini P. L'acetato di idrocortisone nel trattamento della sindrome sciatalgica. Minerva Med 1954; 1:1863-1865.

Bigos SJ, Boyer OR, Braen GR et al. Acute Low Back Problems in Adults. Clinical Practice Guideline Number 4. AHCPR Publication No. 95-0642. Agency for Health Care Policy and Research, Public Health Service, US Department of Health and Human Services, Rockville, December 1994.

Biondi J, Greenberg BJ. Redecompression and fusion in failed back syndrome patients. J Spinal Disord 1990; 3:362-369.

Blomberg R. A method for spinal canal endoscopy and spinaloscopy: Presentation of preliminary results. Acta Anesth Scand 1985; 21:113-116.

Bobechko WT, Hircsch C. Autoimmune response to nucleus pulposus in the rabbit. J Bone Joint Surg 1965; 47B:574-580.

Bogduk N, Aprill C. On the nature of neck pain, discography, and cervical zygapophyseal joint blocks. Pain 1993; 54:213217.

Bogduk N, Christophidis N, Cherry D et al. Epidural use of steroids in the management of back pain. Report of working party on epidural use of steroids in the management of back pain. National Health and Medical Research Council. Canberra, Commonwealth of Australia, 1994, pp 1-76.

Bogduk N, Long DM. Percutaneous lumbar medial branch neurotomy. A modification of facet denervation. Spine 1980; 5:193-200.

Bogduk N, Macintosh J, Marsland A. Technical limitations to the efficacy of radiofrequency neurotomy for spinal pain. $\mathrm{Neu}$ rosurgery 1987; 20:529-535.

Bogduk N, Marsland A. On the concept of third occipital headache. J Neurol Neurosurg Psychiatry 1986; 49:775-780.

Bogduk N, Marsland A. The cervical zygapophyseal joints as a source of neck pain. Spine 1988; 13:610-617.

Bogduk N, Modic MT. Controversy: Lumbar discography. Spine 1996; 21:402-404.
Bogduk N, Simons DG. Neck pain: Joint pain or trigger points. In: H Vaeroy and J Merskey (eds). Progress in Fibromyalgia and Myofascial Pain. Elsevier, Amsterdam, 1993, pp 267-273.

Bogduk N, Wilson AS, Tynan W. The human lumbar dorsal rami. J Anat 1982; 134:383-397.

Bogduk N. Diskography. APS J 1994; 3:149-154.

Bogduk N. Epidural steroids for low back pain and sciatica. Pain Digest 1999; 9:226-227.

Bogduk N. International Spinal Injection Society guidelines for the performance of spinal injection procedures. Part 1: Zygapophyseal joint blocks. Clin J Pain 1997; 13:285-302.

Bogduk N. Musculoskeletal pain: Toward precision diagnosis. Progress in pain research and management. In Jensen TS, Turner JA, Wiesenfeld-Hallin Z (eds). Proceedings of the 8th World Congress on Pain. IASP Press, Seattle, 1997, pp 507525.

Bogduk N. Prelude to a discussion on discography, towards an acceptable and convincing standard of practice. ISIS $8 \mathrm{TH}^{\mathrm{Tn}}$ nual Scientific Meeting, San Francisco, Sept. 2000.

Bogduk N. The argument for discography. Neurosurgery Quarterly 1996; 6:152-153.

Bogduk N. The clinical anatomy of the cervical dorsal rami. Spine 1982; 7:35-45.

Bogduk N. The innervation of the lumbar spine. Spine 1983; 8:286-293.

Bombardier C, Esmail R, Nachemson AL et al. The Cochrane collaboration back review group for spinal disorders. Spine 1997; 22:837-840.

Bonica JJ, Backup PH, Anderson CE. Peridural block, an analysis of 3,637 cases. A review. Anesthesiology 1957; 18:723734.

Bonica JJ. The management of Pain. Lea \& Febiger, Philadelphia, 1953.

Bonica JJ. Definitions and taxonomy of pain. In Bonica JJ, Loessor JD, Chapman CR et al (eds). The Management of Pain, Second Edition. Lea \& Febiger, Philadelphia, 1990; Vol. 1: pp 18-27.

Boonen S, Van Distel G, Westhovens R et al. Steroid myopathy induced by epidural triamcinolone injection. Brit J Rheumatol 1995; 34:385.

Borenstein D. Prevalence and treatment outcome of primary and secondary fibromyalgia in patients with spinal pain. Spine 1995; 20:796-800.

Borenstein D. Approach to the diagnosis and management of medical low back pain. Semin Spine Surg 1990; 2:80-85.

Bourne IHJ. Treatment of chronic back pain. Comparing corticosteroid-lignocaine injections with lignocaine alone. Practitioner 1984; 228:333-338.

Bovim G, Berg R, Dale LG. Cervicogenic headache: Anesthetic blockades of cervical nerves (C2-C5) and facet joint (C2/C3). Pain 1992; 49:315-320.

Bovim G, Schrader H, Sand T. Neck pain in the general population. Spine 1994; 19:1307-1309.

Bowman SJ, Wedderburn L, Whaley A et al. Outcome assessment after epidural corticosteroid injection for low back pain and sciatica. Spine 1993; 18:1345-1350.

Brechner T. Percutaneous cryogenic neurolysis of the articular nerve of Luschka. Reg Anesth 1981; 6:18-22. 
Breivik H, Hesla PE, Molnar I et al. Treatment of chronic low back pain and sciatica. Comparison of caudal epidural injections of bupivacaine and methylprednisolone with bupivacaine followed by saline. In Bonica JJ, Albe-Fesard D (eds). Advances in pain research and therapy. Raven Press, New York, 1976, Vol. 1, pp 927-932.

Bressler HB, Keyes WJ, Rochon PA et al. The prevalence of low back pain in the elderly. A systemic review of the literature. Spine 1999; 24:1813-1819.

Bridenbaugh LD, Moore DC, Campbell DD. Management of upper abdominal cancer pain. JAMA 1964; 190:877-880.

Broadhurst NA, Bond MJ. Pain provocation tests for the assessment of sacroiliac joint dysfunction. J Spin Disord 1998; 11:341-345.

Bromage PR. Spinal extradural abscess: Pursuit of vigilance. $\mathrm{Br}$ J Anaesth 1993; 70:471-473.

Bromage RP, Benumof JL. Paraplegia following intracord injection during attempted epidural anesthesia under general anesthesia. Reg Anesth Pain Med 1998; 23:104-107.

Brook Robert H. Practice guidelines and practicing medicine. Are they compatible? JAMA 1989; 262:3027-3030.

Brown FW. Management of discogenic pain using epidural and intrathecal steroids. Clin Orthop 1977; 129:72-78.

Brown JH. Pressure caudal anesthesia and back manipulation. Northwest Med (Seattle) 1960; 59:905-909.

Buckwalter JA. Aging and degeneration of the human intervertebral disk. Spine 1995; 20:1307-1314.

Bullard JR, Houghton FM. Epidural treatment of acute herniated nucleus pulposus. Anesth Analg 1977; 56:862-863.

Burcheil KJ, Anderson VC, Brown FD et al. Prospective, multicenter study of spinal cord stimulation for relief of chronic back and extremity pain. Spine 1996; 21:2786-2794.

Burman MS. Myeloscopy or the direct visualization of the spinal cord. J Bone Joint Surg 1931; 13:695-696.

Burn JM, Guyer PB, Langdon L. The spread of solutions injected into the epidural space: A study using epidurograms in patients with lumbosciatic syndrome. Br J Anaesth 1973; 45:338-345.

Burton CV, Kirkaldy-Willis WH, Yong-Hing K et al. Causes of failure of surgery on the lumbar spine. Clin Orthop 1981; 157:191-199.

Burton CV. Causes of failure of surgery on the lumbar spine: Ten-year follow up. Mt Sinai J Med 1991; 58:183-187.

Burton CV. Percutaneous radiofrequency facet denervation. Appl Neurophysiol 1976/77; 39:80-86.

Bush C, Ditto B, Feurstein M. A controlled evaluation of paraspinal EMG biofeedback in the treatment of chronic low back pain. Health Psychol 1985; 4:307-321.

Bush K, Hillier S. A controlled study of caudal epidural injections of triamcinolone plus procaine for the management of intractable sciatica. Spine 1991; 16:572-575.

Bush K, Hillier S. Outcome of cervical radiculopathy treated with periradicular/epidural corticosteroid injections: A prospective study with independent clinical review. Eur Spine J 1996; 5:319-325.

Buss DD, Wright RW. Etiology, diagnosis, and treatment of Paget's disease. Curr Opinion Orthop 1994; 5:26-32.
Cabana MD, Rand CS, Powe NR et al. Why don't physicians follow clinical practice guidelines? A framework for improvement. JAMA 1999; 282:1458-1465.

Campbell JK, Penzien DB, Wall EM. Evidence-based guidelines for migraine headache: Behavorial and physical treatments. 2000. Available at: http://www.aan.com/public/ practiceguidelines/headache_gl.htm.

Campbell JN, Belzberg. Use of disk distension to diagnose pain of spinal origin. APS J 1994; 3:157-159.

Canale L. Il desametazone per via eidurale sacrale nelle lombosciataligie. Gaz Med Ital 1963; 122:210-213.

Cappio M, Fragasso V. Il prednisone per via epidurale sacrale nelle lombosciatalgie. Reumatismo 1957; 5:295-298.

Cappio M, Fragasso V. Osservazioni sull'uso dell'idroscortisone per via eidurale ed endorachidea nelle lombosciatalgie. Riforma Med 1955; 22:605-607.

Cappio M. Il trattamento idrocortisonico per via epidurale sacrale delle lombosciatalgie. Reumatismo 1957; 9:60-70.

Carette S, Lecaire R, Marcoux S et al. Epidural corticosteroid injections for sciatica due to herniated nucleus pulposus. $N$ Engl J Med 1997; 336:1634-1640.

Carette S, Marcoux S, Truchon R et al. A controlled trial of corticosteroid injections into facet joints for chronic low back pain. NEngl J Med 1991; 325:1002-1007.

Carey TS, Garrett JM, Jackman A et al. Recurrence and care seeking after acute back pain. Results of a long-term follow-up study. Medical Care 1999; 37:157-164.

Carey TS. Randomized controlled trials in surgery. An essential component of scientific progress. Spine 1999; 23:2553-2555.

Carragee E, Chen Y, Tanner C et al. Can discography cause longterm back symptoms in previously asymptomatic subjects? Spine 2000; 25:1803-1808.

Carragee E, Tanner C, Khurana S et al. The rates of false-positive lumbar discography in select patients without low back symptoms. Spine 2000; 25:1373-1381.

Carragee EJ, Chen Y, Tanner CM et al. Provocative discography in patients after limited lumbar discectomy. Spine 2000; 25:30653071.

Carragee EJ, Paragioudakis SJ, Khurana S. Lumbar high-intensity zone and discography in subjects without low back problems. Spine 2000; 25:2987-2992.

Carragee EJ, Tanner CM, Yang B et al. False-Positive findings on lumbar discography. Spine 1999; 24:2542-2547.

Carragee EJ. The prevalence and clinical features of internal disc disruption (IDD) in patients with chronic low back pain. Spine 1996; 21:776.

Carrera GF. Lumbar facet joint injection in low back pain and sciatica: Preliminary results. Radiology 1980; 137:665-667.

Carter A. Clinical practice guidelines. Can Med Assoc J 1992; 147:1649-1650.

Cassidy D, Carroll L, Cote P: The Saskatchewan Health and Back Pain Survey. The prevalence of low back pain and related disability in Saskatchewan Adults. Spine 1998; 23:1860-1867. Cassisi JE, Sypert GW, Salamon A et al. Independent evaluation of a multidisciplinary rehabilitation program for chronic low back pain. Neurosurgery 1989; 25:877-883. 
Castagnera L, Maurette P, Pointillart V et al. Long-term results of cervical epidural steroid injection with and without morphine in chronic cervical radicular pain. Pain 1994; 58:239243.

Castro WH, van Akkerveeken PF. Der diagn ostische Wert der selektiven lumbalen Nervenwurzelblockde. A orthop Ihre Grenzgeb 1991; 129:376-379.

Catchlove RFH, Braha R. The use of cervical epidural nerve blocks in the management of chronic head and neck pain. Can Anaesth Soc J1984; 31:188-191.

Cathelin F. Mode d'action de a cocaine injete daus l'escapte epidural par le procede du canal sacre. Comptes Rendies des Senaces de la Societe de Biologic et de ses Filliales, 1901; 43:487.

Caussade G, Queste P. Traitement de al neuralgie sciatique par la mèthode de Sicard. Résultats favorables même dans les cas chroniues par la cocaïne à doses élevées et répétées à intervalles raproches. Bull Soc Med Hosp Paris 1909; 28:865.

Cavanaugh JM, Ozaktay AC, Vaidyanathan S. Mechano- and chemosensitivity of lumbar dorsal roots and dorsal root ganglia: An in vitro study. Trans Orthop Res Soc 1994; 19:109.

Chan ST, Leung S. Spinal epidural abscess following steroid injection for sciatica: Case report. Spine 1989; 14:106-108.

Chang RW, Pellissier JM, Hazen GB. A cost-effectiveness analysis of total hip arthroplasty of osteoarthritis of the hip. JAMA 1996; 275:858-865.

Chaoyang C, Cavanaugh JM, Ozaktay C et al. Effects of phospholipase $\mathrm{A}_{2}$ on lumbar nerve root structure and function. Spine 1997; 22:1057-1064.

Chino N, Awad EA, Kottke FJ. Pathology of propylene glycol administered by perineural and intramuscular injection in rats. Arch Phys Med Rehab 1974; 55:33-38.

Cicala RS, Thoni K, Angel JJ. Long-term results of cervical epidural steroid injections. Clin J Pain 1989; 5:143-145.

Cicala RS, Turner R, Moran E et al. Methylprednisolone acetate does not cause inflammatory changes in the epidural space. Anesthesiology 1990; 72:556-558.

Cicala RS, Wright H. Outpatient treatment of patients with chronic pain. Analysis of cost savings. Clin J Pain 1989; 5:223226.

Ciocon JO, Galindo-Clocon D, Amarnath L et al. Caudal epidural blocks for elderly patients with lumbar canal stenosis. $J \mathrm{Am}$ Geriatr Soc 1994; 42:593-596.

Claeys L. Spinal cord stimulation and chronic critical limb ischemia. Neuromodulation 1999; 2:33-40.

Cleeland CD, Gonin R, Hatfield AK et al. Pain and its treatments in outpatients with metastatic cancer. N Engl J Med 1994; 330:592-596.

Coccharella L, Andersson GBJ (eds). Pain. Guides to the evaluation of permanent impairment. Fifth Edition American Medical Association. AMA press, Chicago, IL, 2000, pp 565-591.

Coderre T. Contribution of protein kinase $\mathrm{C}$ to central sensitization and persistent pain following tissue injury. Neurosci Lett 1992; 140:181-184.

Collee G, Dijkmans B, Cats A et al. Iliac crest pain syndrome in low back pain. A double-blind, randomized study of local injection therapy. J Rheumatol 1991; 18:1060-1063.
Committee to Advise the Public Health Service on Clinical Practice Guidelines, Institute of Medicine. Field MJ, Lohr KN (eds). Clinical Practice Guidelines. Directions of a New Program. National Academy Press; Washington, 1990.

Concato J, Shah N, Horwitz RI. Randomized, controlled trials, observational studies, and the hierarchy of research designs. $N$ Engl J Med 2000; 342:1887-1892.

Cook SD, Prewett AB, Dalton JE et al. Reduction in perineural scar formation after laminectomy with Polyactive ${ }^{\circledR}$ membrane sheets. Spine 1994; 19:1815-1825.

Coomes EN. A comparison between epidural anesthesia and bedrest in sciatica. Brit Med J 1961; 1:20-24.

Cooper R, Freemont A, Hoyland J et al. Herniated intervertebral disc-associated periradicular fibrosis and vascular abnormalities occur without inflammatory cell infiltration. Spine 1995; 20:591-598.

Corran TM, Farrell MJ, Helme RD et al. The classification of patients with chronic pain: Age as a contributing factor. Clin J Pain 1997; 13:207-214.

Côté DC, Cassidy JD, Carroll L. The factors associated with neck pain and its related disability in the Saskatchewan population. Spine 2000; 25:1109-1117.

Côté DC, Cassidy JD, Carroll L. The Saskatchewan Health and Back Pain Survey. The prevalence of neck pain and related disability in Saskatchewan adults. Spine 1998; 23:1689-1698.

Cousins MJ. Pain: The past, present, and future of anesthesiology? The E.A. Rovenstine Memorial Lecture. Anesthesiology 1999; 91:538-551.

Crock HV. A reappraisal of intervertebral disc lesions. Med J Aust 1970; 1:983-989.

Crock HV. Isolated lumbar disc resorption as a cause of nerve root canal stenosis. Clin Orthop 1976; 115:109-115.

Croft PR, Papageorgiou AC, Thomas E et al. Short-term physical risk factors for new episodes of low back pain. Prospective evidence from the South Manchester Back Pain Study. Spine 1999; 24:1556-1561.

Crombie IK, Croft PR, Linton SJ et al (eds). Epidemiology of pain: A report of task force on epidemiology of the International Association for the Study of Pain. IASP Press, Seattle 1999.

Cuckler JM, Bernini PA, Wiesel SW et al. The use of epidural steroid in the treatment of radicular pain. J Bone Joint Surg 1985; 67:63-66.

Czarski Z. Leczenie rwy kulszowej wstrzykiwaniem hydrokortyzonu inowokainy do rozworu kryzowego. Przeglad Kekarski 1965; 21:511-513.

D'Hoogue R, Compere A, Gribmont B et al. Peridural injection of corticosteroids in the treatment of the low back pain/sciatica syndrome. Acta Orthop Belg 1976; 42:157-165.

Dahl JL. Improving the practice of pain management. JAMA 2000; 284:2785.

Daly P. Caudal epidural anesthesia in lumbosciatic pain. Anesthesia 1970; 25:346-348.

Davis H. Increasing rates of cervical and lumbar spine surgery in the United States, 1979-1990. Spine 1994; 19:1117-1124. 
Day LJ, Bovill EG, Trafton PG et al. Orthopedics. In Way LW (ed). Current surgical diagnosis and treatment. Appleton Lange, Connecticut, pp 1011-1104.

de Jong RH. BACKLASH: AHCPR practice guideline for acute low back pain. Pain Digest 1996; 6:1-2.

De La Porte C, Van de Kelft E. Spinal cord stimulation in failed back surgery syndrome. Pain 1993; 52:55-61.

Delaney TJ, Rowlingson JC, Carron $\mathrm{H}$ et al. Epidural steroid effects on nerves and meninges. Anesth Analg 1980; 58:610614.

Derby R, Eek B, Chen Y et al. Intradiscal electrothermal annuloplasty (IDET): A novel approach for treating chronic discogenic back pain. Neuromodulation, 2000; 3(2):82-88.

Derby R, Howard M, Grant J et al. The ability of pressure controlled discography to predict surgical and non-surgical outcome. Spine 1999; 24:364-371.

Derby R, Kine G, Saal JA et al. Precision percutaneous blocking procedures for localizing spinal pain. Part 2: The lumbar neuraxial compartment. Pain Digest 1993; 3:175-188.

Derby R, Kine G, Saal JA et al. Response to steroid and duration of radicular pain as predictors of surgical outcome. Spine 1992; 17 (Suppl): 176-183.

Derby R, O’Neill CW. The reported effects on referred leg pain post intradiscal electrothermal therapy. Proceedings of International Spinal Injection Society, San Francisco, September 810, 2000.

Derby R. Cervical epidural steroid injection with intrinsic spinal cord damage. Point of view. Spine 1998; 23:2141-2142.

Desoutet JM, Gilula LA, Murphy WA et al. Lumbar facet joint injection: Indication, technique, clinical correlation, and preliminary results. Radiology 1982; 145:321-325.

Devor M, Govrin-Lippmann R, Raber P. Corticosteroids suppress ectopic neural discharges originating in experimental neuromas. Pain 1985; 22:127-137.

Devulder J, Laat MD, Bastelaere MV. Spinal cord stimulation: A valuable treatment for chronic failed back surgery patients. $J$ Pain Symptom Manage; 1997; 13:296-301.

Devulder J, Lutgarde B, Castille F et al. Relevance of epidurography and epidural adhesiolysis in chronic failed back surgery patients. Clin J Pain 1995; 11:147-150.

Devulder J. Transforaminal nerve root sleeve injection with corticosteroids, hyaluronidase, and local anesthetic in the failed back surgery syndrome. J Spinal Disord 1998; 11:151-154.

Deyo RA, Rainville J, Kent DL. What can the history and physical examination tell us about low back pain? JAMA 1992; 268:760-765.

Deyo RA. Fads in the treatment of low back pain. $N$ Engl J Med 1991; 325:1039-1040.

Dickersin K, Manheimer E. The Cochrane collaboration: Evaluation of health care and services using systematic reviews of the results of randomized controlled trials. Clin Obstet Gynecol 1998; 41(2):315-331.

Dilke TFW, Burry HC, Grahame R. Extradural corticosteroid injection in the management of lumbar nerve root compression. Br Med J 1973; 2:635-637.

Doleys DM, Coleton M, Tutak U. Use of intraspinal infusion therapy with non-cancer pain patients. Follow up and comparison of worker's compensation versus non-worker's compensation patients. Neuromodulation 1998; 1:149-159.

Donaldson S, Romney D, Donaldson M et al. Randomized study of the application of single motor unit biofeedback training to chronic low back pain. J Occup Rehabil 1994; 4:23-37.

Donovan M, Dillon P, McGuire L. Incidence and characteristics of pain in a sample of medical-surgical inpatients. Pain 1987; 30:69-87.

Dooley JF, McBroom RJ, Taguchi T et al. Nerve root infiltration in the diagnosis of radicular pain. Spine 1988; 13:79-83.

Dory MA. Arthrography of the cervical facet joints. Radiology 1983; 148:379-382.

Dory MA. Arthrography of the lumbar facet joints. Radiology 1981; 140:23-27.

Dougherty JH, Fraser RAR. Complications following intraspinal injections of steroids. J Neurosurg 1978; 48:1023-1025.

Drew B, Bhandari M, Kulkarni AV et al. Reliability in grading the severity of lumbar spinal stenosis. J Spinal Disord 2000; 13:253-258.

Dreyfuss P, Halbrook B, Pauza K et al. Efficacy and validity of radiofrequency neurotomy for chronic lumbar zygapophysial joint pain. Spine 2000; 25:1270-1277.

Dreyfuss P, Michaelsen M, Fletcher D. Atlanto-occipital and lateral atlanto-axial joint pain patterns. Spine 1994; 19:11251131.

Dreyfuss P, Michaelsen M, Pauza K et al. The value of medical history and physical examination in diagnosing sacroiliac joint pain. Spine 1996; 21:2594-2602.

Dreyfuss P, Schwarzer AC, Lau P et al. Specificity of lumbar medial branch and L5 dorsal ramus blocks: A computed tomography study. Spine 1997; 22:895-902.

Dreyfuss P, Tibiletti C, Dreyer S et al. Thoracic zygapophyseal joint pain: A review and description of an intra-articular block techniques. Pain Digest 1994; 4:46-54.

Dreyfuss P, Tibiletti C, Dreyer SJ. Thoracic zygapophyseal joint pain patterns: A study in normal volunteers. Spine 1994; 19:807-811.

Dreyfuss P. Dreyer S. Lumbar facet joint injections. In Gonzalez EG, Materson RS (eds). The Nonsurgical Management of Acute Low Back Pain. Demos vermande, New York 1997, pp 123136.

Dusault DG, Nicolet VM. Cervical facet joint arthrography. $J$ Can Assoc Radiol 1985; 36:79-80.

Dwyer A, Aprill C, Bogduk N. Cervical zygapophyseal joint pain patterns: A study in normal volunteers. Spine 1990; 6:453-457.

Ebraheim NA, Mekhail AO, Wiley WF et al. Radiology of the sacroiliac joint. Spine 1997; 22:869-876.

Eddy DM. Comparing benefits and harms: The balance shifts. JAMA 1990; 263:2493-2501.

Eisenberg E, Carr DB, Chalmers TC. Neurolytic celiac plexus block for treatment of cancer pain: A meta-analysis. Anesth Analg 1995; 80:290-295.

Eisenberg JM. Clinical economics: A guide to the economic analysis of clinical practices. JAMA 1989; 262:2879-2886. 
Elias M. Cervical epidural abscess following trigger point injection. J Pain Symptom Manage 1994; 9:71-72.

Elias M. Cervical sympathetic and stellate ganglion blocks. Pain Physician 2000; 3:294-304.

Elias M. The anterior approach for thoracic sympathetic ganglion block using a curved needle. Pain Clinic 2000; 12:17-24.

El-Khoury G, Ehara S, Weinstein JW et al. Epidural steroid injection: A procedure ideally performed with fluoroscopic control. Radiology 1988; 168:554-557.

Elves MW, Bucknill T, Sullivan MF. In vitro inhibition of leucocyte migration in patients with intervertebral disc lesions. Orthop Clin North Am 1975; 6:59-65.

Epstein RS, Sherwood LM. From outcomes research to disease management: A guide for the perplexed. Ann Intern Med 1996; 124:832-837.

Erdine S, Ozyalcin S. Comments on arachnoiditis following epidural adhesiolysis. Pain Digest 1997; 7:158-159.

Espeland A, Korsbrekke K, Albreksten G et al. Observer variation in plain radiography of the lumbosacral spine. Brit J Rad 1998; 71:366-375.

Esses SI. The diskography dilemma. APS J 1994; 3:155-156.

Evans W. Intrasacral epidural injection in the treatment of sciatica. Lancet 1930; 2:1225-1229.

Ezzo J, Berman B, Hadhazy VA et al. Is acupuncture effective for the treatment of chronic pain? A systematic review. Pain 2000; 86:217-225.

Faber LE, Wakim NG, Duhring JL. Evolving concepts in the mechanism of steroid action: Current developments. Am JObstet Gynecol 1987; 156:1449-1458.

Fager CA, Freidberg SR. Analysis of failures and poor results of lumbar spine surgery. Spine 1980; 5:87-94.

Fairbank J. Randomized controlled trials in the surgical management of spinal problems. Spine 1999; 23:2556-2563.

Fairbank JCT, Park WM, McCall IW et al. Apophyseal injection of local anesthetic as a diagnostic aid in primary low back pain syndromes. Spine 1981; 6:598-605.

Fanuele JC, Birkmeyer NJ, Abdu WA et al. The impact of spinal problems on the health status of patients. Have we underestimated the effect? Spine 2000; 25:1509-1514.

Farrell MJ, Gibson SJ, Helme RD. Chronic nonmalignant pain in older people. In Ferrell BR, Ferrell BA, (eds). Pain in the elderly. IASAP Press, Seattle 1996, pp 81-89.

Ferraz MB, Maetzel A, Bombardier C. A summary of economic evaluations published in the field of rheumatology and related disciplines. Arthr Rheum 1997; 40:1587-1593.

Ferrell BA, Ferrell BR, Osterwell D. Pain in the nursing home. $J$ Am Geriatr Soc 1990; 38:409-414.

Ferrell BR, Ferrell BA (eds). Pain in the elderly. A report of the task force on pain in the elderly of the international association for the study of pain. IASP Press, Seattle, 1996.

Fine PG, Milano R, Hare BD. The effects of myofascial trigger point injections are naloxone reversible. Pain 1988; 32:15-20.

Fink BR, Cairns AM. Differential use-dependent (frequencydependent) effects in single mammalian axons: Data and clinical considerations. Anesthesiology 1987; 67:477-484.

Fishbain D, Cutler RB, Rosomoff HL et al. What is the quality of the implemented meta-analytic procedures in chronic pain treatment meta-analyses? Clin J Pain 2000; 16:73-85.

Foley KM. The treatment of cancer pain. N Engl J Med 1985; 313:84-95.

Forrest JB. The response to epidural steroid injections in chronic dorsal root pain. Can Anaesth Soc J 1980; 27:40-46.

Forrest JB. Management of chronic dorsal root pain with epidural steroids. Can Anesth Soc J 1978; 25:218.

Fortin JD, Aprill CN, Ponthieux B et al. Sacroiliac joints: Pain referral maps upon applying a new injection/arthrography technique. Part II: Clinical evaluation. Spine 1994; 19:1483-1489.

Fortin JD, Dwyer AP, West S et al. Sacroiliac joint: pain referral maps upon applying a new injection/arthrography technique. Part I: Asymptomatic volunteers. Spine 1994; 19:1475-1482.

Fortin JD. Precision diagnostic disc injections. Pain Physician 2000; 3:271-288.

Fowler FJ. Using patient reports to evaluate medical outcomes. United States Congress, Office of Technology Assessment. Tools for Evaluating Health Technologies. Background Paper Five, BP-H-142. U.S. Government Printing Office, Washington, February, 1995.

Fowler RJ, Blackwell GJ. Anti-inflammatory steroid induced biosynthesis of a phospholipase $\mathrm{A}_{2}$ inhibitor which prevents prostaglandin generation. Nature 1979; 278:456-459.

Fox AJ, Melzack R. Transcutaneous electrical stimulation to acupuncture. Comparison of treatment of low back pain. Pain 1976; 2:141-148.

Fragasso V. Il prednisolone idrosolubile per via epidurale sacrale nelle lombosciatalgie. Gaz Med Ital 1959; 118:358-360.

Fredman B, Nun MB, Zohar E et al. Epidural steroids for treating "failed back surgery syndrome": Is fluoroscopy really necessary? Anesth Analg 1999; 88:367-372.

Fredman B, Zohar E, Ben Nun BM, Iraqi R et al. The effect of repeated epidural sympathetic nerve block on "failed back surgery syndrome" associated chronic low back pain. JClin Anesth 1999; 11:46-51.

Fritsch EW, Heisel J, Rupp S. The failed back surgery syndrome. Reasons, intraoperative findings, and long-term results: A report of 182 operative treatments. Spine 1996; 21:626-633.

Frost FA, Jessen B, Singgarrd-Andersen J. A control doubleblind comparison of mepivacaine injection versus saline injection for myofascial pain. Lancet 1980; 8167-8168.

Frymoyer JW. Lumbar disk disease: Epidemiology. Instr Course Lect 1992; 41:217-223.

Frymoyer JW. Magnitude of the problem. In Weinstein J, Weisel SW (eds). The Lumbar Spine. WB Saunders, Philadelphia, 1990, pp 32-38.

Fujiwara A, Lim T, An H et al. The effect of disc degeneration and facet joint osteoarthritis on the segmental flexibility of the lumbar spine. Spine 2000; 25:3036-3044.

Fujiwara A, Tamai K, An HS. The relationship between disc degeneration, facet joint osteoarthritis, and stability of the degenerative lumbar spine. J Spinal Disord 2000; 13:444-450.

Fujiwara A, Tamai K, Yamato M et al. The relationship between facet joint osteoarthritis and disc degeneration of the lumbar spine: An MRI study. Eur J Spine 1999; 8:396-401. 
Fukui S, Ohseto K, Shiotani M et al. Distribution of referral pain from the lumbar zygapophyseal joints and dorsal rami. Clin J Pain 1997; 13:303-307.

Fukui S, Ohseto K, Shiotani M et al. Referred pain distribution of the cervical zygapophyseal joints and cervical dorsal rami. Pain 1996; 68:79-83.

Fukusaki M, Kobayashi I, Hara T et al. Symptoms of spinal stenosis do not improve after epidural steroid injection. Clin J Pain 1998; 14:148-151.

Fukusaki M, Tsuji K, Fujie T et al. The effects of blockade of the facet joint of the atlas and axis upon muscle tension headaches. Headache 1990; 4:312-317.

Furman MB, O'Brien EM, Zgleszewski TM. Incidence of intravascular penetration in transforaminal lumbosacral epidural steroid injections. Spine 2000; 25:2628-2632.

Gallagher J, Vadi PLP, Wesley JR. Radiofrequency facet joint denervation in the treatment of low back pain - A prospective controlled double-blind study to assess efficacy. Pain Clinic 1994; 7:193-198.

Garvey TA, Marks MR, Wiesel SW. A prospective, randomized, double-blind evaluation of trigger-point injection therapy for low-back pain. Department of Orthopedic Surgery, The George Washington University Medical Center, Washington, DC, and The Cleveland Clinic, Cleveland, Ohio, 1989, pp 962964.

Gerest MF. Le traitement de la nevralgie sciatique par les injections epidurales d'hydrocortisone. JMed Lyon 1958; 261-264.

Gertzbein SD, Tait JH, Devlin SR. The stimulation of lymphocytes by nucleus pulposus in patients with degenerative disk disease of the lumbar spine. Clin Orthop 1977; 123:149-154.

Gertzbein SD, Tile M, Gross A. Autoimmunity in degenerative disc disease of the lumbar spine. Orthop Clin North Am 1975; 6:67-73.

Gertzbein SD. Degenerative disk disease of the lumbar spine: Immunological implications. Clin Orthop 1977; 129:68-71.

Geurts JW, Stolker RJ. Percutaneous radiofrequency lesion of the stellate ganglion in the treatment of pain in upper extremity reflex sympathetic dystrophy. Pain Clinic 1993; 6:17-25.

Gibson JNA, Grant IC, Waddell G. The Cochrane review of surgery for lumbar disc prolapse and degenerative lumbar spondylosis. Spine 1999; 24:1820-1832.

Gilly R. essai de traitement de 50 cas de sciatiques et de radiculalgies lombaires par le Clestene chronodose en infiltrations paradiculaire. Marseille Medicale 1970; 107:341-345.

Gladstone JC, Pennant JH. Spinal anaesthesia following facet joint injection. Anaesthesia 1987; 42:754-756.

Godlee F. Clinical evidence. BMJ Publishing Group, London, June 2000; pp 486-572.

Goebert HW, Jallo SJ, Gardner WJ et al. Painful radiculopathy treated with epidural injections of procaine and hydrocortisone acetate results in 113 patients. Anesth Analg 1961; 140:130134.

Goldberg R, Genant H, Shimshak R et al. Applications and limitations of quantitative sacroiliac joint scintigraphy. Radiology 1978; 128:683-686.

Gonzalez EG, Materson (eds). The nonsurgical management of acute low back pain. Demos Vermane, New York 1997.
Goossens MEGJB, Evers SMA. Economic evaluation of back pain interventions. J Occup Rehab 1997; 7:15-32.

Goossens MEJB, Evers SMAA, Vlaeyen JWS et al. Principles of economic evaluation for interventions of chronic musculoskeletal pain. Eur J Pain 1999; 3:343-353.

Gordon J. Caudal extradural injection for the treatment of low back pain. Anaesthesia 1980; 35:515-516.

Goucke CR, Graziotti P. Extradural abscess following local anaesthetic and steroid injection for chronic low back pain. Brit J Anesth 1990; 65:427-429.

Grant DJ, Bishop-Miller J, Winchester DM, et al. A randomized comparative trial of acupuncture versus transcutaneous electrical nerve stimulation of chronic back pain in the elderly. Pain 1999; 82:9-13.

Greenfield S, Kaplan SH, Silliman RA et al. The uses of outcomes research for medical effectiveness, quality of care, and reimbursement in Type II diabetes. Diabetes Care 1994; 17:3239.

Greenman PE. Clinical aspects of sacroiliac function in walking. JMan Med 1990; 5:25-130.

Grönblad M, Virri J, Seitsalo S et al. Inflammatory cells, motor weakness, and straight leg raising in transligamentous disc herniations. Spine 2000; 25:2803-2807.

Grönblad M, Virri J, Tolonen J et al. A controlled immunohistochemical study of inflammatory cells in disc herniation tissue. Spine 1994; 19:2744-2751.

Guo HR, Tanaka S, Halperin WE et al. Back pain prevalence in US industry and estimates of lost work days. Am J Public Health 1999; 89:1029-1035.

Gupta AK, Singh R, Shukla RK et al. Role of epidural medication in the treatment of resistant low back pain. J Indian Med Assoc 1996; 94:224-226, 233.

Gureje O, Von Korff M, Simon GE et al. Persistent pain and well being: A World Health Organization Study in Primary Care. JAMA 1998; 280:147-151.

Gutknecht DR. Chemical meningitis following epidural injections of corticosteroids (Letter). Am J Med 1987; 82:570.

Guyer RD, Ohnmeiss DD. Contemporary concepts in spine care. Lumbar discography. Position statement from the North American Spine Society and Therapeutic Committee. Spine 1995; 18:2048-2059.

Hagen KB, Hilde G, Jamtvedt $\mathrm{G}$ et al. The Cochrane review of bed rest for acute low back pain and sciatica. Spine 2000; 25:2932-2939.

Haldeman S. Failure of the pathological model to predict back pain. Spine 1990; 15:718-732.

Hameroff SR, Crago BR, Blitt CD et al. Comparison of bupivacaine, etidocaine, and saline for trigger point therapy. Anesth Analg 1981; 60:752-755.

Hammer M, Meneese W. Principles and practice of radiofrequency neurolysis. Cur Rev Pain 1998; 2:267-278.

Han SC, Harrison P. Myofascial Pain Syndrome and TriggerPoint Management. Reg Anesth 1997; 22(1):89-101.

Handel JA, Knap J, Poletti S. The structural degenerative cascade. The cervical spine. In White AH, Schofferaian, JA (eds). Spine Care. Diagnosis and conservative treatment. Mosby, St. Louis, 1995, Vol. 1: pp 16-23. 
Hanley EN Jr. The cost of surgical intervention for lumbar disc herniation. In Weinstein JN (ed). Clinical efficacy and outcome in the diagnosis and treatment of low back pain. Raven Press, New York, 1992, pp 125-133.

Hanley EN, Shapiro DE. The development of low back pain after excision of a lumbar disc. J Bone Joint Surg 1989; 71A:719721.

Hanly JG, Mitchell MJ, Barnes DC et al. Early recognition of sacroiliitis by magnetic resonance imaging and single photon emission computed tomography. J Rheum 1994; 21:2088-2095.

Hansson TH, Hansson EK. The effects of common medical interventions on pain, back function, and work resumption in patients with chronic low back pain. Spine 2000; 25:30553064.

Harden RN. A clinical approach to complex regional pain syndrome. Clin J Pain (Suppl) 2000; 16:S26-32.

Harley C. Extradural corticosteroid infiltration. A follow-up study of 50 cases. Ann Phy Med 1966; 9:22-28.

Harrington JF, Messier AA, Bereiter D et al. Herniated lumbar disc material as a source of free glutamate available to affect pain signals through the dorsal root ganglion. Spine 2000; 25:929936.

Harris JS. Development, use, and evaluation of clinical practice guidelines. JOEM 1997; 39:23-34.

Hartman JT, Winnie AP, Ramaurthy S et al. Intradural and extradural corticosteroids for sciatica pain. Orthop Rev 1974; 3:2124.

Hassenbusch SJ, Stanton-Hicks M, Covington EC et al. Longterm intraspinal infusion of opiates in the treatment of neuropathic pain. J Pain Symptom Manage 1995; 10:527-543.

Hatten HP Jr. Lumbar epidurography with metrizamide. Radiology 1980; 137:129-136.

Haueisen DC, Smith BS, Myers SR et al. The diagnostic accuracy of spinal nerve injection studies. Clin Orthop Rel Res 1985; 198:179-183.

Hayashi N, Weinstein JN, Meller ST et al. The effect of epidural injection of betamethasone or bupivacaine in a rat model of lumbar radiculopathy. Spine 1998; 23:877-885.

Hazard RG, Reid S, Haugh LD et al. A controlled trial of an educational pamphlet to prevent disability after occupational low back injury. Spine 2000; 25:1419-1423.

Heavner JE, Chokhavatia S, Kizelshteyn G. Percutaneous evaluation of the epidural and subarachnoid space with the flexible fiberscope. Reg Anesth 1991; 15S1:85.

Heavner JE, Racz GB, Raj P. Percutaneous epidural neuroplasty. Prospective evaluation of $0.9 \% \mathrm{NaCl}$ versus $10 \% \mathrm{NaCl}$ with or without hyaluronidase. Reg Anesth Pain Med 1999; 24:202207.

Heavner JE. Comments on arachnoiditis following epidural adhesiolysis. Pain Digest 1997; 7:157.

Hegedus V. Relief of pancreatic pain by radiography-guided block. Am J Radiol 1979; 133:1101-1103.

Helbig T, Lee CK. The lumbar facet syndrome. Spine 1988; 13:6164.

Helliwell M, Robertson JC, Ellia RM. Outpatient treatment of low back pain and sciatica by a single extradural corticosteroid injection. Br J Clin Pract 1985; 39:228-231.
Helme RD, Gibson SJ. Pain in older people. In Crombie IK, Croft PR, Linton SJ et al (eds). Epidemiology of pain. IASP Press; Seattle, 1999, pp 103-112.

Hendler NH, Bergson C, Morrison C. Overlooked physical diagnoses in chronic pain patients involved in litigation. Part 2. Psychosomatics 1996; 37:509-517.

Hendler NH, Kolodny AL. Using medication wisely in chronic pain. Patient Care 1992; May 15:125.

Herron LD. Selective nerve root block in patient selection for lumbar surgery: Surgical results. J Spinal Disord 1989; 2:7579.

Heyse-Moore GH. A rational approach to the use of epidural medication in the treatment of sciatic pain. Acta Orthop Scand 1978; 49:366-370.

Hickey RF. Outpatient epidural steroid injections for low back pain and lumbosacral radiculopathy. NZ Med J 1987; 100:5459.

Hildebrandt J, Argyrakis A. Percutaneous nerve block of the cervical facets - A relatively new method in the treatment of chronic headache and neck pain. Man Med 1986; 2:48-52.

Hill CS. When will adequate pain treatment be the norm? JAMA 1995; 274:1881-1882.

Hilten BJV, Willem-Johan T, Beek VD et al. Intrathecal baclofen for the treatment of dystonia in patients with reflex sympathetic dystrophy. N Engl J Med 2000; 343:625-630.

Hirsch C, Schajowicz F, Galante J. Structural changes in the cervical spine. Acta Orth Scand 1967; 109:68-72.

Hirsch C. An attempt to diagnose level of disc lesion clinically by disc puncture. Acta Orthop Scand 1948; 18:131-140.

Hirsch D, Inglemark B, Miller M. The anatomical basis for low back pain. Acta Orthop Scand 1963; 33:1.

Hitchcock ER, Prandini MN. Hypertonic saline in management of intractable pain. Lancet 1973; 1:310-312.

Hodges SD, Castleberg RL, Miller T et al. Cervical epidural steroid injection with intrinsic spinal cord damage. Two case reports. Spine 1998; 23:2137-2142.

Hodgson PSA, Mack B, Kopacz D et al. Needle placement during lumbar epidural anesthesia deviates toward the non-dependent side (abstract). Reg Anesth 1996; 21:26.

Hoffmann DE. Pain management and palliative care in the era of managed care: Issue for health insurers. J Law, Med \& Ethics 1998; 26:267-289.

Hogan QH. Epidural anatomy examined by cryomicrotome section. Influence of age, vertebral level and disease. Reg Anesth 1996; 21:295-306.

Holt E. Fallacy of cervical discography. JAMA 1964; 188:799801.

Hoppenstein R. A new approach to the failed back syndrome. Spine 1980; 5:371-379.

Hopwood M. Outcomes assessment in pain management. In Abram SE (ed). Pain Management. Churchill-Livingston, Philadelphia, 1998, pp 14.1-14.11.

Hopwood MB, Manning DC. Lumbar epidural steroid injections. Reg Anesth Pain Med 1999; 24:5-7.

Horlocker TT, Wedel DJ, Offord KP. Does preoperative antiplatelet therapy increase the risk of hemorrhagic complications associated with regional anesthesia? Anesth Analg 1990; 70:631-634 
Hove B, Glydensted C. Cervical analgesia facet joint arthrography. Neuroradiology 1990; 32:456-459.

Hua SY, Chen YZ. Membrane receptor-mediated electrophysiological effects of glucocorticoid on mammalian neurons. Endocrinology 1989; 124:687-691.

Hurwitz EL, Aker PD, Adams AH et al. Manipulation and mobilization of the cervical spine. A systematic review of the literature. Spine 1996; 21:1746-1760.

Ignelzi RJ, Cummings TW. A statistical analysis of percutaneous radiofrequency lesions in the treatment of chronic low back pain and sciatica. Pain 1980; 8:181-187.

Isaacsson A, Hanson BS, Ranstam J et al. Social network, social support, and the prevalence of neck and low back pain after retirement. A population study of men born in 1914 in Malmö, Sweden. Scand J Soc Med 1995; 23:17-22.

Ischia S, Ischia A, Polati E et al. Three posterior percutaneous celiac plexus block techniques. Anesthesiology 1992; 76:534540.

Ito R. The treatment of low back pain and sciatica with epidural corticosteroids injection and its pathophysiologic basis. J Jpn Orthop Assoc 1971; 45:769-777.

Jackson DW, Rettig A, Wiltse LL. Epidural cortisone injection in the young athletic adult. Am J Sports Med 1980; 8:239-243.

Jackson RP, Jacobs RR, Montesano PX. Facet joint injection in low back pain. A prospective study. Spine 1988; 13:966-971.

Jackson RP. The facet syndrome. Myth or reality? Clin Orthop 1992; 279:110-121.

Jacobs A., Pullan PT, Potter JM et al. Adrenal suppression following extradural steroids. Anaesthesia 1983; 38:953-956.

Jacobs MJ, Jorning PJ, Joshi SR et al. Epidural spinal cord electrical stimulation improves microvascular blood flow in severe limb ischaemia. Ann Surg 1988; 207:179-183.

Jacobson L, Mariano A, Chabal C et al. Beyond the needle. Expanding the role of anesthesiologists in the management of chronic non-malignant pain. Anesthesiology 1997; 87:12101218.

Jadad AR, Carroll D, Moore A et al. Developing a database of published reports of randomized clinical trials in pain research. Pain 1996; 66:239-246.

Jaffray D, O'Brien JP. Isolated intervertebral disc resorption: A source of mechanical and inflammatory back pain? Spine 1986; 11:397-401.

Jalali S. Epidural endoscopic adhesiolysis: A valuable technique in treating refractory low back and extremity pain. $A m J$ Anesthesiol 2000; 27:261-264.

Jamison RN, VadeBoncouer T, Ferrante FM. Low back pain patients unresponsive to an epidural steroid injection: Identifying predictive factors. Clin J Pain 1991; 7:311-317.

Jensen IB, Bodin L, Ljungqvist T et al. Assessing the needs of patients in pain: A matter of opinion? Spine 2000; 25:28162823.

Jensen TS, Krebs B, Nielsen J et al. Immediate and long-term phantom limb pain in amputees: incidence, clinical characteristics and relationship to -pre-amputation limb pain. Pain 1985; 21:267-278.

Johansson A, Bennett GJ. Effect of local methylprednisolone on pain in a nerve injury model. A pilot study. Reg Anesth 1997;
22:59-65.

Johansson A, Hao J, Sjolund B. Local corticosteroid application blocks transmission in normal nociceptor C-fibers. Acta Anaesthesiol Scand 1990; 34:335-338.

Jurmand SH. Cortiotherapie peridurale des lombalgies et des sciatiques d'origine discale. Concours Medicale 1972; 94:50615070 .

Kalauokalani DAK, Loeser JD. Phantom limb pain. In Crombie IK, Croft PR, Linton SJ et al (eds). Epidemiology of pain. IASP Press, Seattle, 1999, pp 143-153.

Kang JD, Georgescu HI, McIntyre-Larkin L et al. Herniated lumbar intervertebral discs spontaneously produce matrix metalloproteinases, nitric oxide, interleukin-6, and prostaglandin $\mathrm{E}_{2}$. Spine 1996; 21:271-277.

Kaplan M, Dreyfus P, Halbrook B et al. The ability of lumbar medial branch blocks to anesthetize zygapophysial joint. Spine 1998; 23:1847-1852.

Karasek M, Bogduk N. Twelve-month follow-up of a controlled trial of intradiscal thermal annuloplasty for back pain due to internal disc disruption. Spine 2000; 25:2601-2607.

Katsaros GA, Handjis GP, Anastasiades J. Forty-eight singleshot epidural injections on the same patient. Anaesthesia 1990; 45:381-382.

Katz JA, Lukin R, Bridenbaugh PO et al. Subdural intracranial air: An unusual cause of headache after epidural steroid injection. Anesthesiology 1991; 74:615.

Katz SS, Savitz MH. Percutaneous radiofrequency rhizotomy of the lumbar facets. Mt Sinai J Med 1986; 7:523-525.

Kaul S, Meena AK, Sundaram C et al. Spinal extradural abscess following local steroid injection. Neurol India 2000; 48:181183.

Kawakami M, Tamaki T, Hashizume H et al. The role of phospholipase $\mathrm{A}_{2}$ and nitric oxide in pain-related behavior produced by an allograft of intervertebral disc material to the sciatic nerve of the rat. Spine 1997; 22:1074-1079.

Kawakami M, Tamaki T, Weinstein JN et al. Pathomechanism of pain-related behavior produced by allografts of intervertebral disc in the rat. Spine 1996; 21:2101-2107.

Kawakami M, Weinstein JN, Tamaki, et al. The difference in nociceptive potential of the nucleus pulposus and the anulus fibrosus. In Weinstein JN, Gordon SL (eds). Low Back Pain: A Scientific and Clinical Overview. American Academy of Orthopaedic Surgeons, 1996, Rosemont, pp 209-213.

Kayama S, Konno S, Olmarker K et al. Incision of the annulus fibrosus induces nerve root morphologic, vascular, and functional changes. Spine 1996; 21:2539-2543.

Keller RB, Atlas SJ, Soule DN et al. Relationship between rates and outcomes of operative treatment for lumbar disc herniation and spinal stenosis. J Bone Joint Surg 1999; 81A(6):752-762.

Kellgren JH. The anatomical source of back pain. Rheumatol Rehab 1977; 16:3-12.

Kemler MA, Barendse G, van Kleef M et al. Spinal cord stimulation in patients with chronic reflex sympathetic dystrophy. NEngl J Med 2000; 343:618-624.

Kepes ER, Duncalf D. Treatment of backache with spinal injections of local anesthetics, spinal and systemic steroids. Pain $1985 ; 22: 33-47$. 
Keskimaki I, Seitsalo S, Osterman H et al. Reoperations after lumbar disc surgery. A population-based study of regional and interspecialty variations. Spine 2000; 25:1500-1508.

Kikuchi S, Hasue M, Nishiyama K. Anatomic and clinical studies of radicular symptoms. Spine 1984; 9:23-30.

Kikuchi S. Anatomical and experimental studies of nerve root infiltration. JJPN Orthop Assoc 1982; 56:27-36.

Kim RC, Porter RW, Choi BH et al. Myelopathy after intrathecal administration of hypertonic saline. Neurosurgery 1988; 22:942-944.

King JS, Lagger R. Sciatica viewed as a referred pain syndrome. Surg Neurol 1976; 5:46-50.

Kingery WS, Castellote JM, Maze M. Methylprednisolone prevents the development of autotomy and neuropathic edema in rats, but has no effect on nociceptive thresholds. Pain 1999; 80:555-566.

Kirkaldy-Willis WH, Wedge JH, Yong-Hing K et al. Pathology and pathogenesis of lumbar spondylosis and stenosis. Spine 1978;3:319-327.

Klein RG, Vaccaro AR, Cwik J et al. Efficacy of cervical epidural steroids in the treatment of cervical spine disorders. $\mathrm{Am} \mathrm{J}$ Anesthesiol 2000; (9):547-552.

Klekamp J, McCarty E, Spengler DM. Results of elective lumbar discectomy for patients involved in the workers' compensation system. J Spinal Disord 1998; 11:277-282.

Klenerman L, Greenwood R, Davenport HT et al. Lumbar epidural injections in the treatment of sciatica. Br J Rheumatol 1984; 23:35-38.

Knight CL, Burnell JC. Systemic side-effects of extradural steroids. Anaesthesia 1980; 35:593-594.

Knight JW, Cordingley JJ, Palazzo MGA. Epidural abscess following epidural steroid and local anaesthetic injection. Anaesthesia 1997; 52:576.

Koes BW, Assendelft WJJ, Heijden GJMG et al. Spinal manipulation and mobilization for back and neck pain: A blinded review. Br Med J 1991; 303:1298-1303.

Koes BW, Bouter LM, Mameren H V, et al. A blinded randomized clinical trial of manual therapy and physiotherapy for chronic back and neck complaints: Physical outcome measures. J Manipulative Physiol Ther 1992; 1:16-23.

Koes BW, Bouter LM, Van Der Heijden GJMGV. Methodological quality of randomized clinical trials on treatment efficacy in low back pain. Spine 1995; 20:228-235.

Koes BW, Hoogen HMMV. Efficacy of bed rest and orthoses for low back pain: A review of randomized clinical trials. Eur $J$ Phys Med Rehab 1994; 4:86-93.

Koes BW, Scholten R, Mens JMA et al. Epidural steroid injections for low back pain and sciatica. An updated systematic review of randomized clinical trials. Pain Digest 1999; 9:241247.

Koes BW, Scholten RJPM, Mens JMA et al. Efficacy of epidural steroid injections for low back pain and sciatica: A systematic review of randomized clinical trials. Pain 1995; 63:279288.

Koning HM, Mackie DP. Percutaneous radiofrequency facet denervation in low back pain. Pain Clinic 1994; 7:199-204.
Kraemer J, Ludwig J, Bickert U et al. Lumbar epidural perineural injection: A new technique. Eur Spine J 1997; 6:357-361.

Krames E. Spinal cord stimulation: Indications, mechanism of action, and efficacy. Cur Rev Pain 1999; 3:419-426.

Krames ES, Lanning RM. Intrathecal infusional analgesia for non-malignant pain: analgesic efficacy of intrathecal opioids with and without bupivacaine. J Pain Symptom Manage 1993; 8:539-548.

Krames ES, Schuchard M. Implantable intraspinal infusion analgesia: Management guidelines. Pain Rev 1995; 2:243-267.

Krane EJ, Dalens BJ, Murat I, et al. The safety of epidurals during general anesthesia. Reg Anesth Pain Med 1998; 23:433438.

Krempen JF, Smith BS. Nerve root injection: A method for evaluating the etiology of sciatica. J Bone Joint Surg [AM] 1974; 56A:1435-1444.

Kumar K, Nath R, Wyant GM. Treatment of chronic pain by epidural spinal cord stimulation: A 10-year experience. $J$ Neurosurg 1991; 75:402-407.

Kuntz K, Snider R, Weinstein J et al. Cost-effectiveness of fusion with and without instrumentation for patients with degenerative spondylolisthesis and spinal stenosis. Spine 2000; 25:1132-1139.

Kuslich SD, Ulstrom CL, Michael CJ. The tissue origin of low back pain and sciatica: A report of pain response to tissue stimulation during operation on the lumbar spine using local anesthesia. Orthop Clin North Am 1991; 22:181-187.

Lantto T. The scintigraphy of sacroiliac joints: A comparison of 99-m Tc-VPB and 99mTc-MDP. Eur J Nucl Med 1990; 16:677681.

LaRocca H. Failed lumbar surgery. Principles of management. In Weinstein J, Wiesel S (eds). The Lumbar Spine. WB Saunders Company, Philadelphia, 1990, pp 872-881.

Latham JM, Fraser RD, Moore RJ et al. The pathologic effects of intrathecal betamethasone. Spine 1997; 22:1558-1562.

Lau LS, Littlejohn GO, Miller MH. Clinical evaluation of intraarticular injections for lumbar facet joint pain. Med J Aust 1985; 143:563-565.

Lave JR, Frank RG, Schulberg HC et al. Cost effectiveness of treatments for major depression in primary care practice. Arch Gen Psychiatry 1998; 55:645-651.

Lavsky-Shulan M, Wallace RB, Kohout FJ et al. Prevalence and functional correlates of low back pain in the elderly. The Iowa 65+ Rural Health Study. J Am Geriatr Soc 1985; 33:23-28.

Law JD, Lehman RAW, Kirch WM. Reoperation after lumbar intervertebral disc surgery. J Neurosurg 1978; 48:259-263.

Lawrence RC, Helmick CG, Arnett FC. Estimates of the prevalence of arthritis and selected musculoskeletal disorders in the United States. Arthritis \& Rheumatism 1998; 41:778-799.

Leape LL. Practice guidelines and standards: An overview. $Q R B$ Qual Rev Bull 1990; 16:42-49.

Leape LL. Park RE, Solomon DH et al. Does inappropriate use explain small-area variations in the use of health care services? JAMA 1990; 263:669-672.

Lee HM, Weinstein JN, Meller ST et al. The role of steroids and their effects on phospholipase $\mathrm{A}_{2}$. An animal model of radiculopathy. Spine 1998; 23:1191-1196. 
Lee J, Lutz GE, Campbell D et al. Stability of the spine after intradiscal electrothermal therapy. Proceedings of the International Spinal Injection Society, $5^{\text {th }}$ Annual Scientific Meeting, Las Vegas, August 13-15, 1999.

Lees F, Turner JWA. Natural history and prognosis of cervical spondylosis. Br Med J 1963; 2:1607-1610.

Lehmann LJ, Pallares VS. Subdural injection of a local anesthetic with steroids: Complication of epidural anesthesia. South Med $J$ 1995; 88:467-469.

Leigh JP, Markowitz S, Fahs M et al. Occupational injury and illness in the United States. Estimates of costs, morbidity, and mortality. Arch Intern Med 1997; 157:1557-1568.

Lewandowski EM. The efficacy of solutions used in caudal neuroplasty. Pain Digest 1997; 7:323-330.

Lewinnek GE, Warfield CA. Facet joint degeneration as a cause of low back pain. Clin Orthop 1986; 213:216-222.

Li Yue-Ming, Wingrove DE, Too HP et al. Local anesthetics inhibit substance $P$ binding and evoked increases in intracellular $\mathrm{Ca}^{2+}$. Anesthesiology 1995:82:166-173

Lievre JA, Block-Michel H, Attali P. L'injection transscree etude clinique et radiologrique. Bull Soc Med Hop 1957; 73:11101118 .

Lilius G, Laasonen EM, Myllynen P et al. Lumbar facet joint syndrome. A randomized clinical trial. J Bone Joint Surg (Br) 1989; 71:681-684.

Lindblom K. Protrusions of the discs and nerve compression in the lumbar region. Acta Radiol Scand 1944; 25:195-212.

Lindhal O, Rexed B. Histologic changes in spinal nerve roots of operated cases of sciatica. Acta Orthop Scand 1951; 20:215225.

Lindner A, Warmuth-Metz M, Becker G et al. Iatrogenic spinal epidural abscesses: Early diagnosis essential for good outcome. Eur JMed Res 1997; 2:201-205.

Lindström I, Öhlund C, Eek C et al. Mobility, strength, and fitness after a graded activity program for patients with subacute low back pain: A randomized prospective clinical study with a behavioral therapy approach. Spine 1992; 17:641-652.

Lindström I, Öhlund C, Eek C et al. The effect of graded activity on patients with subacute low back pain: A randomized prospective clinical study with an operant-conditioning behavioral approach. Phys Ther 1992; 72:279-293.

Ling C, Atkinson PL, Munton CGF. Bilateral retinal hemorrhages following epidural injection. Br J Ophthalmol 1993; 77:316.

Linton SJ, Hellsing AL, Hallden K. A population based study of spinal pain among 35-45-year old individuals. Spine 1998; 23:1457-1463.

Lippitt AB. The facet joint and its role in spine pain. Management with facet joint injections. Spine 1984; 9:746-750.

Liu B, Manos R, Criscitiello A et al. Clinical factors associated with favorable outcomes using intradiscal electrothermal modulation (IDET). Proceedings of Spine Across the Sea, Hawaii, July 23-27, 2000.

Lord SM, Barnsley L, Bogduk N. Percutaneous radiofrequency neurotomy in the treatment of cervical zygapophyseal joint pain: a caution. Neurosurgery 1995; 35:732-739.
Lord SM, Barnsley L, Bogduk N. The utility of comparative local anesthetic blocks versus placebo-controlled blocks for the diagnosis of cervical zygapophysial joint pain. Clin J Pain 1995; 11:208-213.

Lord SM, Barnsley L, Wallis BJ et al. Chronic cervical zygapophysial joint pain with whiplash: A placebo-controlled prevalence study. Spine 1996; 21:1737-1745.

Lord SM, Barnsley L, Wallis BJ et al. Percutaneous radio-frequency neurotomy for chronic cervical zygapophyseal-joint pain. N Eng J Med 1996; 335:1721-1726.

Lord SM, Barnsley L, Wallis BJ et al. Third occipital nerve headache. A prevalence study. JNeurol Neurosurg Psychiatry 1994; 57:1187-1190.

Lou L, Racz GB, Heavner JE. Percutaneous epidural neuroplasty. In Waldman SD (ed). Interventional Pain Management. Second Edition, WB Saunders Company, Philadelphia, 2000, pp 434445.

Lubenow T, Keh-Wong E, Kristof K et al. Inadvertent subdural injection: A complication of an epidural block. Anesth Analg 1988; 67:175-179.

Lucas JS, Ducker TB, Perot PL. Adverse reactions to intrathecal saline injections for control of pain. JNeurosurg 1975; 42:57561.

Lutz GE, Vad VB, Wisneski RJ. Fluoroscopic transforaminal lumbar epidural steroids: An outcome study. Arch Phys Med Rehabil 1998; 79:1362-1366.

Lynch MC, Taylor JF. Facet joint injection for low back pain. A clinical study. J Bone Joint Surg (Br) 1986; 68:138-141.

Lynn J, Virnig BA. Assessing the significance of treatment effects: Comments from the perspective of ethics. Med Care 1995; 33:AS292-AS298.

Macfarlane GJ, Hunt IM, McBeth J et al. Chronic widespread pain in the community: Influences on health-care seeking behavior. J Rheumatol 1999; 26:413-419.

Macfarlane GJ. Fibromyalgia and chronic widespread pain. In epidemiology of pain. Crombie IK, Croft PR, Linton SJ et al (eds), IASP Press, Seattle, 1999, pp 113-124.

MacKinnon Se, Hudson AR, Gentilli R et al. Peripheral nerve injection injury with steroid agents. Plast Reconstr Surg 1982; 69:482-489.

Macnab I. Negative disc exploration: An analysis of the causes of nerve root involvement in sixty-eight patient. J Bone Joint Surg [AM] 1971; 53A:5891-5903.

Maetzel A, Ferraz, MB, Bombardier C. A review of cost-effectiveness analyses in rheumatology and related disciples. Current Opin Rheumatol 1998; 10:136-140.

Magni G, Caldieron C, Rigatti-Luchini S et al. Chronic musculoskeletal pain and depressive symptoms in the general population. An analysis of the $1^{\text {st }}$ National Health and Nutrition Examination Survey Data. Pain 1990; 43:299-307.

Maigne JY, Aivakiklis A, Pfefer F: Results of sacroiliac joint double block and value of sacroiliac pain provocation test in 54 patients with low back pain. Spine 1996; 21:1889-1892.

Maigne JY, Boulahdour H, Charellier G. Value of quantitative radionuclide bone scanning in the diagnosis of sacroiliac joint syndrome in 32 patients with low back pain. Eur Spine J 1998; 7:328-331. 
Malmqvist EL, Bengtsson M, Sorenson J. Efficacy of stellate ganglion block: A clinical study with bupivacaine. Reg Anesth 1992; 17:340-347.

Malter AD, Larwon EB, Urban N et al. Cost-effectiveness of lumbar discectomy for the treatment of herniated intervertebral disc. Spine 1996; 21:1048-1055.

Mam MK. Results of epidural injection of local anaesthetic and corticosteroid in patients with lumbosciatic pain. J Indian Med Assoc 1995; 93:17-18, 24.

Mamourian AC, Dickman CA, Drayer BP et al. Spinal epidural abscess: Three cases following spinal epidural injection demonstrated with magnetic resonance imaging. Anesthesiology 1993; 78:204.

Manchikanti L, Bakhit CE, Pakanati RR et al. Fluoroscopy is medically necessary for the performance of epidural steroids. Anesth Analg 1999; 89:1326-1327.

Manchikanti L, Bakhit CE, Pampati V. Role of epidurography in caudal neuroplasty. Pain Digest 1998; 8:277-281.

Manchikanti L, Bakhit CE. Percutaneous lysis of epidural adhesions. Pain Physician 2000; 3:46-64.

Manchikanti L, Bakhit CE. Removal of torn Racz catheter from lumbar epidural space. Reg Anesth 1997; 22:579-581.

Manchikanti L, Pakanati RR, Bakhit CE, et al. Non-endoscopic and endoscopic adhesiolysis in post lumbar laminectomy syndrome. A one-year outcome study and cost effectiveness analysis. Pain Physician 1999; 2:52-58.

Manchikanti L, Pakanati RR, Pampati V. Comparison of three routes of epidural steroid injections in low back pain. Pain Digest 1999; 9:277-285.

Manchikanti L, Pakanati RR, Pampati V. The value and safety of epidural endoscopic adhesiolysis. Am J Anesthesiol 2000; 27:275-279.

Manchikanti L, Pampati V, Bakhit CE et al. Effectiveness of lumbar facet joint nerve blocks in chronic low back pain: A randomized clinical trial. Pain Physician 2001; 4: 101-117.

Manchikanti L, Pampati V, Fellows B et al. The inability of the clinical picture to characterize pain from facet joints. Pain Physician 2000; 3:158-166.

Manchikanti L, Pampati VS, Bakhit CE et al. The diagnostic validity and therapeutic value of lumbar facet joint nerve blocks with or without adjuvant agents. Cur Rev Pain 2000; 4:337344.

Manchikanti L, Pampati VS, Beyer C et al. The effect of neuraxial steroids on weight and bone mass density: A prospective evaluation. Pain Physician 2000; 3:357-366.

Manchikanti L, Pampati VS, Fellows B et al. Prevalence of lumbar facet joint pain in chronic low back pain. Pain Physician 1999; 2:59-64.

Manchikanti L, Singh V, Bakhit CE et al. Interventional techniques in the management of chronic pain: Part 1.0. Pain Physician 2000; 3:7-42.

Manchikanti L. Chronic low back pain in the elderly: Part 1. AJPM 1997; 7:104-117.

Manchikanti L. Chronic low back pain in the elderly: Part 2. AJPM 1997; 7:133-145.

Manchikanti L. Comments on arachnoiditis following epidural adhesiolysis. Pain Digest 1997; 7:157-158.
Manchikanti L. Dr. No. Managed care: Clinical guidelines. Pain Physician 2000; 3:3-6.

Manchikanti L. Epidemiology of low back pain. Pain Physician 2000; 3:167-192.

Manchikanti L. Epidural steroid injections and intrinsic spinal cord damage. Spine 1999; 24:1170-1171.

Manchikanti L. Evaluation and management services in interventional pain practice: Doing it right! Pain Physician 2000; 3:322341.

Manchikanti L. Facet joint pain and the role of neural blockade in its management. Cur Rev Pain 1999; 3:348-358.

Manchikanti L. Neural blockade in cervical pain syndromes. Pain Physician, 1999; 2:65-84.

Manchikanti L. The role of neural blockade in the management of chronic low back pain. Pain Digest 1999; 9:166-181.

Manchikanti L. The role of radiofrequency in the management of complex regional pain syndrome. Cur Rev Pain 2000; 4:437444.

Manchikanti L. The value and safety of steroids in neural blockade: Part 1. AJPM 2000; 10:69-78.

Manchikanti L. The value and safety of steroids in neural blockade: Part 2. AJPM 2000; 10:121-133.

Manchikanti L. Transforaminal lumbar epidural steroid injections. Pain Physician 2000; 3:374-398.

Manchikanti L. Pakanati RR, Bakhit CE et al. Role of adhesiolysis and hypertonic saline neurolysis in management of low back pain. Evaluation of Modification of Racz Protocol. Pain Digest, 1999; 9:91-96.

March LM, Skinner J, Schwatz J et al. Musculoskeletal disability in an elderly population living independently. A random survey in the northern Sydney area. Aust NZ J Med 1992; 22:731.

Marks R, Semple AJ. Spinal anaesthesia after facet joint injection. Anaesthesia 1988; 43:65-66.

Marks R. Distribution of pain provoked from lumbar facet joints and related structures during diagnostic spinal infiltration. Pain 1989; 39:37-40.

Marks RC, Houston T, Thulbourne T. Facet joint injection and facet nerve block. A randomized comparison in 86 patients with chronic low back pain. Pain 1992; 49:325-328.

Marks RM, Sachar EJ. Undertreatment of medical patients with narcotic analgesics. Ann Intern Med 1973; 78:173-181.

Maron J, Loeser JD. Spinal opioid infusion in treatment of chronic pain of non-malignant origin. Clin J Pain 1996; 12:174179.

Marshall LL, Trethewie ER, Curtain CC. Chemical radiculitis: A clinical, physiological and immunological study. Clin Orthop 1977; 129:61-67.

Marwick C. Will evidence-based practice help span the gulf between medicine and law? JAMA 2000; 283:2775-2778.

Mateo E, Lopez-Alarcon MD, Moliner S et al. Epidural and subarachnoid pneumocephalus after epidural technique. Eur $J$ Anesethsiol 1999; 16:413-17.

Matthews JA, Mills SB, Jenkins VM et al. Back pain and sciatica: Controlled trials of manipulation, traction, sclerosant and epidural injections. Brit J Rheumatol 1987; 26:416-423. 
Maurer P, Schlemback D, Brown M. Lumbar intradiscal electrothermal annuloplasty (IDEA) for discogenic low back pain. Proceedings of International Intradiscal Therapy Society Annual Meeting, Williamsburg, June 8-10, 2000.

Mayer T, McMahon MJ, Gatchel RJ et al. Socioeconomic outcomes of combined spine surgery and functional restoration in workers' compensation spinal disorders with matched controls. Spine 1998; 23:598-606.

Mayer TG, Polatin P, Smith B et al. Spine rehabilitation. Secondary and tertiary nonoperative care. Spine 1995; 20:20602066.

McCall IW, Park WM, O'Brien JP. Induced pain referral from posterior elements in normal subjects. Spine 1979; 4:441-446.

McCarron RF, Wimpee MW, Hudkins PG et al. The inflammatory effects of nucleus pulposus: A possible element in the pathogenesis of low back pain. Spine 1987; 12:760-764.

McCauley JD, Thelen MH, Frank RG et al. Hypnosis compared with relaxation in the outpatient management of chronic low back pain. Arch Phys Med Rehabil 1983; 64:548-552.

McCormick CC. Arthrography of the atlanto-axial (C1-C2) joints: Technique and results. J Intervent Radiol 1987; 2:9.

McDonald GJ, Lord SM, Bogduk N. Long-term follow-up of patients treated with cervical radiofrequency neurotomy for chronic neck pain. Neurosurgery 1999; 45:61-68.

McEvoy GK, Litvak K, Welsh OH et al. Adrenals. AHFS 99 drug information. American Society of Health-System Pharmacists, Bethesda, 1999, pp 2636-2662.

McPherson K, Strong PM, Epstein A et al. Regional variations in the use of common surgical procedures: Within and between England and Wales, Canada and the United States of America. Soc Sci Med 1981; 15A:273-288.

McQuay H, Moore A. An evidence-based resource for pain relief. Oxford University Press. Oxford, New York, 1998.

McQuay H, Moore A. Finding all the relevant trials. An evidence based resource for pain relief. Oxford University Press, Oxford, New York, 1998, pp 5-9.

McQuay H, Moore A. Introduction: An evidence-based resource for pain relief. Oxford University Press, Oxford, New York, 1998, pp 1-4.

McQuay HJ, Moore RA. Epidural corticosteroids for sciatica. An Evidence-Based Resource for Pain Relief. Oxford University Press, Oxford, New York, 1998; pp 216-218.

Mehta M, Salmon N. Extradural block. Confirmation of the injection site by X-ray monitoring. Anaesthesia 1985; 40:10091012.

Mehta M, Sluijter ME. The treatment of chronic pain. Anesthesia $1979 ; 34: 768-775$.

Meijne W, Van Neerbos K, Aufdemkampe G et al. Intraexaminer and interexaminer reliability of the Gillet test. JMan Phys Ther 1999; 22:4-9.

Melby JC. Drug spotlight program: Systemic corticosteroid therapy; pharmacologic and endocrinologic considerations. Ann Intern Med 1974; 81:505-512.

Melton LJ, Lane AW, Cooper C et al: Prevalence and incidence of vertebral deformities. Osteoporosis Int 1993; 3:113-119.

Melzack R, Wall PD. Pain mechanisms: A new theory. Science 1965; 150:971-978.
Menefee LA, Cohen MJ, Anderson WR et al. Sleep disturbance and nonmalignant chronic pain: A comprehensive review of the literature. Pain Med 2000; 1:156-172.

Mercadante $S$. Celiac plexus block versus analgesics in pancreatic cancer pain. Pain 1993; 52:187-192.

Merskey H, Bogduk N. Classification of chronic pain: Descriptions of chronic pain syndromes and definitions of pain terms. Second Edition. IASP Press, Seattle, 1994.

Miaskowski C, Nichols R, Brody R et al. Assessment of patient satisfaction utilizing the American Pain Society's quality assurance standards on acute and cancer-related pain. J Pain Sym Manage 1994; 9:5-11.

Michelsen JJ, Mixter WJ. Pain and disability of shoulder and arm due to herniation of the nucleus pulposus of cervical intervertebral discs. N Engl J Med 1944; 231:279.

Miedema HS, Chorus AMJ, Wevers CWJ et al. Chronicity of back problems during working life. Spine 1998; 23:2021-2029.

Mikhail Gr, Livingood Cs, Mellinger RC et al. Effect of longacting parenteral corticosteroids on adrenal function. Arch Dermatol 1969; 100:263-268.

Mikhail GR, Sweet LC, Mellinger RC. Parenteral long-acting corticosteroid effect on hypothalamic pituitary adrenal function. Ann Allergy 1973; 31:337-343.

Minamide A, Tamaki T, Hashizume $\mathrm{H}$ et al. Effects of steroids and lipopolysaccharide on spontaneous resorption of herniated intervertebral discs. An experience study in the rabbit. Spine 1998; 23:870-876.

Mironer YE, Somerville JJ. Protocol for diagnosis and treatment of facet joint pain syndrome. A modified three-step approach. Pain Digest 1999; 9:188-190.

Mixter WJ, Barr JS. Rupture of the intervertebral disc with involvement of the spinal canal. N Eng J Med 1934; 211:210215.

Mobily PR, Herr KA, Clark MK et al. An epidemiologic analysis of pain in the elderly. The Iowa $65+$ Rural Health Study. $J$ Aging Health 1994; 6:139-154.

Moneta GB, Videman T, Kaivanto K et al. Reported pain during lumbar discography as a function of anular ruptures and disc degeneration: A re-analysis of 833 discograms. Spine 1994; 17:1968-1974.

Mooney V, Robertson J. The facet syndrome. Clin Orthop 1976; 115:149-156.

Moran R, O'Connell D, Walsh MG. The diagnostic value of facet joint injections. Spine 1986; 12:1407-1410.

Morley S, Eccleston C, Williams A. Systematic review and meta-analysis of randomized controlled trials of cognitive behavior therapy for chronic pain in adults, excluding headache. Pain 1999; 90:1-13.

Mount HTR. Epidural injection of hydrocortisone for the management of the acute lumbar disc protrusion. In Morley TP (ed). Current Controversies in Neurosurgery. WB Saunders, Philadelphia, 1976, pp 67-72.

Mueller-Schwefe G, Hassenbusch SJ, Reig E. Cost-effectiveness of intrathecal therapy for pain. Neuromodulation 1999; 2:77-84. 
Mugford HK. Conflicting conclusions from two systematic reviews of epidural steroid injections for sciatica: Which evidence should general practitioners heed? Br J Gen Pract 1999; 49:57-61.

Murphy RW. Nerve roots and spinal nerves in degenerative disk diseases. Clin Orthop 1977; 129:46-60.

Murtagh FR. Computed tomography and fluoroscopy guided anesthesia and steroid injection in facet syndrome. Spine 1988; 13:686-689.

Nachemson A. Newest knowledge of low back pain: A critical look. Clin Orthop Rel Res 1992; 279:8-20.

Nachemson AL, Vingard E. Assessment of patients with neck and back pain: A best-evidence synthesis. In Nachemson AL, Jonsson E (eds). Neck and Back Pain. The scientific evidence of causes, diagnosis and treatment. Lippincott Williams \& Wilkins, New York, 2000, pp 189-236.

Nachemson AL. Failed back surgery syndrome is syndrome of failed back surgeons. Pain Clinic 1999; 11:271-284.

Nash TP. Current guidelines in the use of epidural steroids in the United Kingdom. Pain Digest 1999; 9:231-232.

Nash TP. Facet joints. Intra-articular steroids or nerve blocks? Pain Clinic 1990; 3:77-82.

Neilson KD, Adams JE, Hosobuchi Y. Experience with dorsal column stimulation for relief of chronic intractable pain: 19681973. Surg Neurol 1975; 4:148-152.

Newton-John T, Spence SH, Schotte D. Cognitive-behavioral therapy versus EMG biofeedback in the treatment of chronic low back pain. Behav Res Ther 1995; 33:691-697.

Nicol GD, Klingberg DK, Vasko MR. Prostaglandin E 2 enhances calcium conductance and stimulates release of substance P in avian sensory neurons. J Neurosci 1992; 12:1917-1927.

Nishimura N, Khahara T, Kusakabe T. The spread of lidocaine and 1-131 solution in the epidural space. Anesthesiology 1959; 20:785-788.

North RB, Campbell JN, James CS et al. Failed back surgery syndrome: 5 year follow-up in 102 patients undergoing repeated operation. Neurosurgery 1991; 28:685-691.

North RB, Ewend MG, Lawton MT et al. Failed back surgery syndrome: 5-year follow-up after spinal cord stimulator implantation. Neurosurgery 1991; 28:692-699.

North RB, Ewend MG, Lawton MT et al. Spinal cord stimulation for chronic, intractable pain: Superiority of "multi-channel" devices. Pain 1991; 44:119-130.

North RB, Han M, Zahurak M et al. Radiofrequency lumbar facet denervation: Analysis of prognostic factors. Pain 1994; 57:77-83.

North RB, Kidd DH, Lee MS et al. A prospective, randomized study of spinal cord stimulation versus reoperation for failed back surgery syndrome: Initial results. Sterotact Funct Neurosurg 1994; 62:267-272.

North RB, Kidd DH, Zahurak M et al. Spinal cord stimulation for chronic intractable pain: experience over two decades. $\mathrm{Neu}$ rosurgery 1993; 32:384-395.

Nouwen A. EMG biofeedback used to reduce standing levels of paraspinal muscle tension in chronic low back pain. Pain 1983; 17:353-360.
Nunn CM. Pathways, guidelines, and cookbook medicine: Are we all becoming Betty Crocker? JCOM 1997; 4:17-24.

Nygaard OP, Mellgren SI, Osterud B. The inflammatory properties of contained and noncontained lumbar disc herniation. Spine 1997; 22:2484-2488.

O'Brien DP, Rawluk DJ. Iatrogenic mycobacterium infection after an epidural injection. Spine 1999; 24:1257-1259.

O’Neill C, Derby R, Kenderes L. Precision injection techniques for diagnosis and treatment of lumbar disc disease. Seminars in Spine Surgery 1999; 11:104-118.

Oakley JC, Weiner RL. Spinal cord stimulation for complex regional pain syndrome: A prospective study of 19 patients at two centers. Neuromodulation 1999; 2:47-50.

Ogoke BA. The management of the atlanto-occipital and atlantoaxial joint pain. Pain Physician 2000; 3:289-293.

Ogsbury JS, Simon RH, Lehman RAW. Facet "denervation" in the treatment of low back syndrome. Pain 1977; 3:257-263.

Ohnmeiss D, Vanharanta H, Ekholm J. Degree of disc disruption and lower extremity pain. Spine 1997; 22:1600-1605.

Okada K. Studies on the cervical facet joints using arthrography of the cervical facet joint. J Jpn Orthop Assoc 1981; 55:563580.

Okuda Y, Suzuki K, Kitajima T et al. Lumbar epidural block for "painful legs and moving toes" syndrome: A report of three cases. Pain 1998; 78:145-147.

Olmarker K, Blomquist J, Stromberg J et al. Inflammatogenic properties of nucleus pulposus. Spine 1995; 20:665-669.

Olmarker K, Byrod G, Cornefijord M et al. Effects of methylprednisolone on nucleus pulposus-induced nerve root injury. Spine 1994; 19:1803-1808.

Olmarker K, Rydevik B, Nordborg C. Autologous nucleus pulposus induces neurophysiologic and histologic changes in porcine cauda equina nerve roots. Spine 1993; 181:1425-1432.

Ombregt L, Ter Veer HJ. Treatment of the lumbar spine. In Ombregt L, Bisschop P, Ter Veer HJ et al (eds). A System of Orthopaedic Medicine. WB Saunders, London, 1995, pp 633688.

Ono K, Ota H, Tada K et al. Cervical myelopathy secondary to multiple spondylotic protrusions: A clinicopathologic study. Spine 1977; 2:109-125.

Onofrio BM, Campa HK. Evaluation of rhizotomy. Review of 12 years experience. J Neurosurg 1972; 36:151-155.

Oudenhoven RC. The role of laminectomy, facet rhizotomy, and epidural steroids. Spine 1979; 4:145-147.

Pages E. Anesthesia metamerica. Rev Sanid Mil Madr 1921; 11:351-385.

Pahor M, Guralnik JM, Wan JY. Lower body osteoarticular pain and dose of analgesic medications in older disabled women: The Women's Health and Aging Study. Am J Public Health 1999; 89:930-934.

Paice JA, Penn RD, Shott S. Intraspinal morphine for chronic non-cancer pain. A retrospective multi-center study. J Pain Symptom Manage 1996; 11:71-80.

Pain. In Guides to the evaluation of permanent impairment. Fourth Edition American Medical Association, Chicago, 1993, pp 303311. 
Pappagallo M, Raja SN, Haytheornwaite JA et al. Oral opioids in the management of postherpetic neuralgia: A prospective survey. Analgesia 1994; 1:51-55.

Parfenchuck T, Janssen M. A correlation of cervical magnetic resonance imaging and discography-computed tomographic discograms. Spine 1994; 19:2819-2825.

Parke WW, Watanable R. Adhesions of the ventral lumbar dura. Adjunct source of discogenic pain? Spine 1990; 15:300-303.

Pasquier NM, Leri D. Injection intra-et extradurales de cocaine a dose minime daus le traitment de la sciatique. Bull Gen Ther $1901 ; 142: 196$.

Pawl RP, Anderson W, Shulman M. Effect of epidural steroids in the cervical and lumbar region on surgical intervention for discogenic spondylosis. In Fields HL (ed). Advances in Pain Research and Therapy. Vol. 9; Raven Press, New York, 1985, pp 791-798.

Perkins HM, Hanlon PR. Epidural injection of local anesthetic and steroids for pain secondary to herpes zoster. Arch Surg 1978; 113:253.

Perquin CW, Hazebroek-Kampschreur AA, Hunfeld JA et al. Chronic pain among children and adolescents: Physician consultation and medication use. Clin J Pain 2000; 16:229-235.

Piccirillo JF. Outcomes research and otolaryngology. Otolaryngol Head Neck Surg 1994; 111:764-769.

Pierce EC. The development of anesthesia guidelines and standards. QRB Qual Rev Bull 1990; 16:61-64.

Pilote L, Califf RM, Sapp S et al. Special articles: Regional variation across the United States in the management of acute myocardial infarction. N Engl J Med 1995; 333:565-572.

Pocock S, Elbourne D. Randomized trials or observational tribulations? (editorial) NEngl J Med 2000; 342:1907-1909.

Porchet F, Vader JP, Larequi-Lauber T et al. The assessment of appropriate indications for laminectomy. J Bone Joint Surg 1999; 81B(62):234-239.

Portenoy RK, Foley KM. Chronic use of opioid analgesics in non-malignant pain. Report of 38 cases. Pain 1986; 25:171186.

Portenoy RK. Opioid therapy for chronic non-malignant pain: Current status. In Nin HI, Fields DS, Liebeskind JC (eds). Pharmacological Approaches to the Treatment of Chronic Pain: Concepts and Critical Issues. IASP Press; Seattle, 1994, pp 247-288.

Portenoy RK. Cancer pain: Epidemiology and syndromes. Cancer 1989; 63:2298.

Postacchini F. Results of surgery compared with conservative management for lumbar disc herniations. Spine 1996; 21:13831387.

Pounder D, Elliott S. An awake patient may not detect spinal cord puncture. Anaesthesia 2000; 55:194.

Practice guidelines for chronic pain management. A report by the American Society of Anesthesiologists Task Force on Pain Management, Chronic Pain Section. Anesthesiology 1997; 86:995-1004.

Purkis IE. Cervical epidural steroids. Pain Clinic 1986; 1:3-7. Quiles M, Marchisello PJ, Tsairis P. Lumbar adhesive arachnoiditis: Ethological and pathological aspects. Spine 1978; 3:4550 .
Rachlin ES. Trigger point management. In Gonzalez EG, Materson RS (eds). The nonsurgical management of acute low back pain. Demos Vermande, New York, 1997, pp 151-158.

Racz GB, Haynsworth RF, Lipton S. Experiences with an improved epidural catheter. Pain Clinic 1986; 1:21-27.

Racz GB, Heavner JE, Diede JH. Lysis of epidural adhesions utilizing the epidural approach. In: Waldman SD, Winnie AP (eds). Interventional Pain Management. WB Saunders, Philadelphia, 1996, pp 339-351.

Racz GB, Heavner JE, Raj PP. Percutaneous epidural neuroplasty. Prospective one-year follow up. Pain Digest 1999; 9:97-102.

Racz GB, Heavner JE, Sigleton W et al. Hypertonic saline and corticosteroid injected epidurally for pain control. In: Racz GB (ed). Techniques of neurolysis. Boston, Kluwer Academic Publishers, 1989, pp 73-94.

Racz GB, Heavner JE. Relevance of epidurography: In response to article by Drs. Devulder et al. Clin J Pain 1995; 11:151-156.

Racz GB, Holubec JT. Lysis of adhesions in the epidural space. In Racz GB (ed). Techniques of Neurolysis. Kluwer Academic Publishers, Boston, 1989, pp 57-72.

Racz GB, Sabonghy M, Gintautas J et al. Intractable pain therapy using a new epidural catheter. JAMA 1982; 248:579-581.

Rainville J, Carlson N, Polatin P. Exploration of physicians' recommendations for activities in chronic low back pain. Spine 2000; 17:2210-2220.

Raja S. Reflex sympathetic dystrophy: Pathophysiological basis for therapy. Pain Digest 1992; 2:274-280.

Rashbaum RF. Radiofrequency facet denervation. A treatment alternative in refractory low back pain with or without leg pain. Orthop Clin North Am 1983; 14:569-575.

Rasmussen BK, Jensen R, Schroll M, et al. Epidemiology of headache in a general population - A prevalence study. J Clin Epidemiol 1991; 44:1147-1157.

Rathmell JP, Song T, Torian D et al. Lumbar epidurography. Reg Anesth Pain Med 2000; 25:540-545.

Raymond J, Dumas JM. Intra-articular facet block: Diagnostic tests or therapeutic procedure? Radiology 1989; 151:333-336.

Reale C, Turkiewicz AM, Reale CA et al. Epidural steroids as a pharmacological approach. Clin Exp Rheumatol 2000; 18:S6566.

Reed KL, Will K. Relevance of epidurography: In response to article by Drs. Devulder et al. Clin J Pain 1995; 11:151-156.

Renfrew DL, Moore TE, Kathol MH et al. Correct placement of epidural steroid injections: Fluoroscopic guidance and contrast administration. Amer J Neuroradiol 1991; 12:1003-1007.

Renier JC. L'infiltration epidurale par le premier trou sacre posterieur. Revue du Rhumatisme et des Maladies Osteoarticulaire 1959; 26:526-532.

Revel M, Auleley GR, Alaoui S et al. Forceful epidural injections for the treatment of lumbosciatic pain with post-operative lumbar spinal fibrosis. Rev Rhum Engl Ed 1996; 63:270277.

Richardson RR, Siqueria EB, Cerullo LJ. Spinal epidural neurostimulation for treatment of acute and chronic intractable pain: Initial and long term results. Neurosurgery 1979; 5:344348. 
Ridley MG, Kingsley GH, Gibson T et al. Outpatient lumbar epidural corticosteroid injection in the management of sciatica. Br J Rheumatol 1988; 27:1003-1007.

Riew KD, Yin Y, Gilula L, et al. Can nerve root injections obviate the need for operative treatment of lumbar radicular pain? A prospective, randomized, controlled, double-blind study. Proceedings of North American Spine Society, 14th Annual Meeting, Chicago. 1999, pp 94-95.

Rivest C, Katz JN, Ferrante FM et al. Effects of epidural steroid injection on pain due to lumbar spinal stenosis or herniated disks: A prospective study. Arthritis Care Res 1998; 11:291297.

Robechhi A., Capra R. L'idrocortisone (composto F). Prime esperienze cliniche in campo reumatologico. Minerva Med 1952; 98:1259-1263.

Roberson GH, Hatten HP Jr, Hesselink JH. Epidurography. Selective catheter technique and review of 53 cases. Am J Radiol 1979; 132:787-793.

Robinson JP, Allen T, Fulton LD et al. Perceived efficacy of pain clinics in the rehabilitation of injured workers. Clin J Pain 1998; 14:202-208.

Rocco AG, Frank E, Kaul AF et al. Epidural steroids, epidural morphine and epidural steroids combined with morphine in the treatment of post-laminectomy syndrome. Pain 1989; 36:297303.

Rogers P, Nash T, Schiller D et al. Epidural steroids for sciatica. The Pain Clinic 1992; 5:67-72.

Roofe PG. Innervation of the anulus fibrosus and posterior longitudinal ligament. Arch Neurol Psych 1940; 44:100-103.

Roos NP, Roos KK. Surgical rate variations. Do they reflect the health or socioeconomic characteristics of the population? Med Care 1982; 20:945-958.

Rose MJ, Reilly JP, Pennie B et al. Chronic low back pain rehabilitation programs: A study of the optimum duration of treatment and a comparison of group and individual therapy. Spine 1997; 22:2246-2253.

Rosen CD, Kahanovitz N, Berstein R et al. A retrospective analysis of the efficacy of epidural steroid injections. Clin Orthop 1988; 228:270-272.

Ross Davies A, Doyle AT, Lansky D et al. Outcomes assessment in clinical settings: A consensus statement on principles and best practices in project management. Jt Comm J Qual Improvement 1994; 20(1):6-16.

Ross JS, Robertson JT, Frederickson RCA et al. Association between peridural scar and recurrent radicular pain after lumbar discectomy: Magnetic resonance evaluation. Neurosurgery 1996; 38:855-863.

Rovenstein EA, Wertheim HM. Therapeutic nerve block. JAMA 1941; 117:1599-1603.

Rowlingson J. Epidural steroids in treating failed back surgery syndrome. Anesth Analg 1999; 88:240-242.

Rowlingson JC, Kirschenbaum LP. Epidural analgesic techniques in the management of cervical pain. Anesth Analg 1986; 65:938942.

Roy DF, Fleury J, Fontaine SB et al. Clinical evaluation of cervical facet joint infiltration. Can Assoc Radiol J 1988; 39:118120.
Rucker KS. Chronic pain evaluation. Butterworth/Heinemann, Boston 2001.

Rutkow IM. Orthopaedic operations in the United States, 19791983. J Bone Joint Surg 1986; 68A:716-719.

Saal JA, Saal JS. Intradiscal electrothermal treatment (IDET) for chronic discogenic low back pain with two year follow-up. Proceedings of North American Spine Society, 15 th Annual Meeting, New Orleans, 2000, pp 5-7.

Saal JA, Saal JS. Intradiscal electrothermal treatment for chronic discogenic low back pain. Spine 2000; 25:2622-2627.

Saal JA. Natural history and non-operative treatment of lumbar disc herniation. Spine 1996; 21:25-35.

Saal JS, Franson RC, Dobrow R et al. High levels of inflammatory phospholipase A2 activity in lumbar disc herniations. Spine 1990; 15:674-678.

Saal JS, Saal JA, Yurth EF. Nonoperative management of herniated cervical intervertebral disc with radiculopathy. Spine 1996; 21:1877-1883.

Saal JS, Saal JA. Management of chronic discogenic low back pain with a thermal intradiscal catheter: A preliminary study. Spine 2000; 25:382-388.

Saberski LR, Ahmad M, Fitzgerald J et al. Practice patterns in the use of epidural corticosteroid injections. A survey of the Connecticut State Society of Anesthesiologists. (Poster Exhibits). Anesthesiology 1998; 101.

Saberski LR, Brull S. Fiberoptic visualization of the spinal cord. A historical review and report of current methods. Yale Biol Med 1995; 68:7-16.

Saberski LR, Kitahata L. Review of the clinical basis and protocol for epidural endoscopy. Connecticut Med 1995; 50:71-73.

Saberski LR, Kitahata LM. Direct visualization of the lumbosacral epidural space through the sacral hiatus. Anesth Analg 1995; 80:839-840.

Saberski LR, Kondamuri S, Osinubi OYO. Identification of the epidural space: Is loss of resistance to air a safe technique? Regional Anesthesia 1997; 22:3-15.

Saberski LR. Spinal endoscopy: current concepts. In Waldman $\mathrm{SD}$ (ed). Interventional Pain Management. Second Edition, WB Saunders Company, Philadelphia, 2000, pp 143-161.

Saberski LR. A retrospective analysis of spinal canal endoscopy and laminectomy outcomes data. A pilot study. Pain Physician 2000; 3:193-196.

Sackett D, Richardson WS, Roseberg W et al. Evidence based medicine. Churchill Livingstone, Philadelphia, 1996.

Sampath P, Rigamonti D. Spinal epidural abscess: A review of epidemiology, diagnosis, and treatment. J Spinal Disord 1999; 12:89-93.

Sandberg DI, Lavyne MH. Symptomatic spinal epidural lipomatosis after local epidural corticosteroid injections: Case report. Neurosurgery 1999; 45:162-165.

Sanders SH, Harden RN, Benson SE et al. Clinical practice guidelines for chronic non-malignant pain syndrome patients II: An evidence-based approach. J Back Musc Rehabil 1999; 13:4758.

Sanders SH, Rucker KS, Anderson KO et al. Clinical practice guidelines for chronic non-malignant pain syndrome patients. $J$ Back Musc Rehabil 1995; 5:115-120. 
Sanders SH. Integrating practice guidelines for chronic pain: From the Tower of Babel to the Rosetta Stone. APS Bulletin 2000; 10(6):1-7.

Savitz MH. Percutaneous radiofrequency rhizotomy of the lumbar facets. Ten years experience. Mt Sinai JMed 1991; 58:177178.

Savolaine ER, Pandya JB, Greenblat SH et al. Anatomy of the human lumbar epidural space: New insights using CTepidurography. Anesthesiology 1988; 68:217-220.

Schaerer JP. Radiofrequency facet rhizotomy in the treatment of chronic neck and low back pain. Int Surg 1978; 63:53-59.

Schaerer JP. Treatment of prolonged neck pain by radiofrequency facet rhizotomy. J Neurol Orthop Med Surg 1988; 9:74-76.

Schellhas KP, Smith MD, Gundry CR et al. Cervical discogenic pain. Spine 1996; 21:300-311.

Scheolkov AP. Evaluation, diagnosis, and initial treatment of cervical disc disease. Spine State of the Art Reviews 1991; 5:167176.

Scher AI, Stewart WF, Lipton RB. Migraine and headache: A meta-analytic approach. In Crombie IK, Croft PR, Linton SJ et al (eds), Epidemiology of pain. IASP Press, Seattle, 1999, pp 159-170.

Schiller F, Shadle OW. Extrathecal and intrathecal suppuration. Arch Neurol 1962; 7:33-36.

Schimmer BP, Parker KL. Adrenocorticotropic hormone; adrenocortical steroids and their synthetic analogs; inhibitors of the synthesis and actions of adrenocortical hormones. In Harman JG, Molinoff PB, Ruddon RW (eds). Goodman's \& Gilman's, The Pharmacological Basis of Therapeutics, Ninth Edition, McGraw-Hill, New York, 1996, pp 1459-1485.

Schmid G, Vetter S, Gottmann D et al. CT-guided epidural/ perineural injections in painful disorders of the lumbar spine: Short- and extended-term results. Cardiovasc Intervent Radiol 1999; 22:493-498.

Schmidt SD, Gibbons JJ. Post dural puncture headache after fluoroscopically guided lumbar paravertebral sympathetic block. Anesthesiology 1993; 78:198-200.

Schuchard M, Lanning R, North R et al. Neurologic sequelae of intraspinal drug delivery systems: Results of a survey of American implanters of implantable drug delivery systems. Neuromodulation 1998; 1:137-148.

Schulz KF, Chalmers I, Hayes RJ et al. Empirical evidence of bias: Dimensions of methodological quality associated with estimates of treatment effects in controlled trials. JAMA 1995; 273:408-412.

Schutz H, Lougheed WM, Wortzman G et al. Intervertebral nerve-root in the investigation of chronic lumbar disc disease. Can J Surg 1973; 16:217-221.

Schwartzman RJ. New treatments for reflex sympathetic dystrophy. N Engl J Med 2000; 343:654-656.

Schwarzer A, Bogduk N. The prevalence and clinical features of internal disc disruption (IDD) in patients with chronic low back pain. Spine 1996; 21:776-777.

Schwarzer AC, Aprill CN, Bogduk M. The sacroiliac joint in chronic low back pain. Spine 1995; 20:31-37.

Schwarzer AC, Aprill CN, Derby R et al. Clinical features of patients with pain stemming from the lumbar zygapophysial joints. Is the lumbar facet syndrome a clinical entity? Spine 1994; 19:1132-1137.

Schwarzer AC, Aprill CN, Derby R et al. The false-positive rate of uncontrolled diagnostic blocks of the lumbar zygapophysial joints. Pain 1994; 58:195-200.

Schwarzer AC, Aprill CN, Derby R et al. The prevalence and clinical features of internal disc disruption in patients with chronic low back pain. Spine 1995; 20:1878-1883.

Schwarzer AC, Aprill CN, Derby R et al. The relative contributions of the disc and zygapophyseal joint in chronic low back pain. Spine 1994; 19:801-806.

Schwarzer AC, Derby R, Aprill CN et al. Pain from the lumbar zygapophysial joints: A test of two models. J Spinal Disord 1994; 7:331-336.

Schwarzer AC, Derby R, Aprill CN et al. The value of the provocation response in lumbar zygapophysial joint injections. Clin J Pain 1994; 10:309-313.

Schwarzer AC, Scott AM, Wang SC et al. The role of bone scintigraphy in chronic low back pain: A comparison of SPECT and planar images and zygapophysial joint injection. Aust NZJ Med 1992; 22:185.

Schwarzer AC, Wang S, Bogduk N et al. Prevalence and clinical features of lumbar zygapophysial joint pain: A study in an Australian population with chronic low back pain. Am Rheum Dis 1995; 54:100-106.

Schwarzer AC, Wang S, Laurent R et al. The role of the zygapophysial joint in chronic low back pain. Aust NZ J Med 1992; 22:185.

Schwarzer AC, Wang SC, O'Driscoll D et al. The ability of computed tomography to identify a painful zygapophysial joint in patients with chronic low back pain. Spine 1995; 20:907912.

Schweitzer A. On the Edge of the Primeval Forest. Macmillan, New York, 1931; 62.

Sehgal AD, Tweed DC, Gardner WS. Laboratory studies after intrathecal corticosteroids. Arch Neurol 1963; 9:64-68.

Sehgal N, Fortin JD. Internal disc disruption and low back pain. Pain Physician 2000; 3:143-157.

Sekel R. Epidural depo-medrol revisited. Med J Aust 1984; 141:688.

Semmes RE, Murphey MF. The syndrome of unilateral rupture of the sixth cervical intervertebral disc with compression of the seventh cervical nerve root. A report of four cases with symptoms simulating coronary disease. JAMA 1943; 121:1209.

Serrao JM, Marks RL, Morley SJ et al. Intrathecal midazolam for the treatment of chronic mechanical low back pain: A controlled comparison with epidural steroid in a pilot study. Pain 1992; 48:5-12.

Shah R, Vad V, Lutz G et al. Transforaminal epidural injections in lumbar radiculopathy; prospective randomized trial. Proceedings of the North American Spine Society, $15^{\text {th }}$ Annual Meeting, New Orleans, 2000, pp 44-45.

Shaneyfelt TM, Mayo-Smith MF, Rothwangl J. Are guidelines following guidelines? The methodological quality of clinical practice guidelines in the peer-reviewed medical literature. JAMA $1999 ; 281: 1900-1905$. 
Sharma RK. Indications, technique and results of caudal epidural injection for lumbar disc retropulsion. Postgrad Med J 1977; 53:1-6.

Sharma S, Stedman R. Epidural steroids. A retrospective analysis of the efficacy of high and low dose therapy. Anesthesiology 1998; 3A:A1135.

Shealy CN, Mortimer JT, Reswick JB. Electrical inhibition of pain by stimulation of the dorsal columns: Preliminary clinical report. Anesth Analg 1967; 46:489-491.

Shealy CN. Dangers of spinal injections without proper diagnosis. JAMA 1966; 197:1104-1106.

Shealy CN. Facet denervation in the management of back sciatic pain. Clin Orthop 1976; 115:157-164.

Shealy CN. Percutaneous radiofrequency denervation of spinal facets. J Neurosurg 1975; 43:448-451.

Shekelle PG, Markovich M, Louie R. An epidemiologic study of episodes of back pain care. Spine 1995; 20:1668-1673.

Shelden CH, Paul F, Jacques DB et al. Electrical stimulation of the nervous system. Surg Neurol 1975; 4:127-132.

Shinomiya K, Nakao N, Shindoh K et al. Evaluation of cervical discography in pain origin and provocation. J Spinal Disord 1993; 6:422-426.

Shoemaker TS. Practice guidelines for pain management. In Ashburn MA and Rice LJ (eds), The Management of Pain. Churchill Livingstone. Philadelphia, 1998, pp 205-226.

Shoemaker TS. Practice policies in anesthesia: A foretaste of practice in the twenty-first century. Anesth Analg 1995; 388403.

Shulman M. Treatment of neck pain with cervical epidural steroid injection. Reg Anesth 1986; 11:92-94.

Sicard MA. Les injections medicamenteuse extraduraqles per voie saracoccygiene. Comptes Renues des Senances de la Societe de Biolgie et de ses Filliales, 1901; 53:396.

Siegfried RN. Development of complex regional pain syndrome after a cervical epidural steroid injection. Anesthesiology 1997; 86:1394-1396.

Simmons DQ, Travell JG. The myofascial genesis of pain. Postgrad Med 1952; 11:425-434.

Simmons E. An evaluation of discography in the localization of symptomatic levels in discogenic diseases of the spine. Clin Orthop 1975; 108:57-59.

Simons DG. Muscular pain syndromes. In Friction JR, Awad EA (eds). Advances in Pain Research and Therapy. LippincottRaven, Philadelphia, 1990, Vol 17.

Simons DG. Myofascial trigger points, a possible explanation. Pain 1988; 69:207-212.

Singh V. Intradiscal electrothermal therapy: A preliminary report. Pain Physician 2000; 3:367-373.

Slipman CW, Jackson HB, Lipetz JL et al. Sacroiliac joint pain referral zones. Arch Phys Med Rehab 2000; 81:334-337.

Slipman CW, Lipetz JS, Jackson HB et al. Therapeutic selective nerve root block in the nonsurgical treatment of atraumatic cervical spondylotic radicular pain: A retrospective analysis with independent clinical review. Arch Phys Med Rehabil 2000; 81:741-746.
Slipman CW, Plastaras CT, Palmitier RA et al. Symptom provocation of fluoroscopically guided cervical nerve root stimulation: Are dynatomal maps identical to dermatomal maps? Spine 1998; 23:2235-2242.

Slipman CW, Sterenfeld EB, Chou LH et al. The predictive value of provocative sacroiliac joint stress maneuvers in the diagnosis of sacroiliac joint syndrome. Arch Phys Med Rehab 1998; 79:288-292.

Slipman CW, Sterenfield EB, Chou LH et al. The value of radionuclide imaging in the diagnosis of sacroiliac joint syndrome. Spine 1996; 21:2251-2254.

Slipman CW. Diagnostic nerve root blocks. In: the nonsurgical management of acute low back pain. Gonzalez EG, Materson RS (eds). Demos vermande, New York 1997: pp 115-122.

Slipman CW. Discography. In Gonzalez EG, Materson RS (eds). The Nonsurgical Management of Acute Low Back Pain. Demos vermande, New York, 1997, pp 103-114.

Slivers RH. Lumbar percutaneous facet rhizotomy. Spine 1990; 15:36-40.

Sluijter ME, Koetsveld-Baart CC. Interruption of pain pathways in the treatment of cervical syndrome. Anaesthesia 1980; 35:302-307.

Sluijter ME, Mehta M. Treatment of chronic back and neck pain by percutaneous thermal lesions. In Lipton S (ed). Persistent Pain, Modern Methods of Treatment. Academic Press, London, 1981, pp 141-179.

Sluijter ME. The use of radiofrequency lesions for pain relief in failed back patients. Int Disabil Studies 1988; 10:37-43.

Sluijter ME. The use of radiofrequency lesions of the communicating ramus in the treatment of low back pain. In Racz GB (ed). Techniques of Neurolysis. Kluwer Academic Publishers; Boston, 1989, pp 145-160.

Smith GW, Robinson RA. The treatment of certain cervical spine disorders by anterior removal of the intervertebral disc and interbody fusion. J Bone Joint Surg [AM] 1958; 40:607623.

Snoek W, Weber H, Jorgensen B. Double-blind evaluation of extradural methylprednisolone for herniated lumbar disc. Acta Orthop Scand 1977; 48:635-641.

Spaccarelli KC. Lumbar and caudal epidural steroid injections. Mayo Clin Proc 1996; 71:169.

Spangfort EV. The lumbar disc herniation. A computer aided analysis of 2504 operations. Acta Orthop Scand (Suppl) 1972; 142:1-95.

Spitzer WO, Leblanc FE, Dupuis M (eds). Quebec Task Force on Spinal Disorders. Scientific approach to the assessment and management of activity-related spinal disorders: A monograph for clinicians. Spine 1987; (suppl) 12:1-59.

Spitzer WO, Skovron ML, Salmi LR et al. Scientific monograph of the Quebec Task Force on whiplash-associated disorders: Redefining "whiplash" and its management. Spine 1995; (Suppl) 20:1-73.

Stanley D, McLaren MI, Euinton HA et al. A prospective study of nerve root infiltration in the diagnosis of sciatica: A comparison with radiculography, computed tomography, and operative findings. Spine 1990; 6:540-543. 
Stanton-Hicks M, Raj P, Racz G. Use of regional anesthetics for diagnosis of reflex sympathetic dystrophy and sympathetically maintained pain; A critical evaluation. In Jänig W, StantonHicks M (eds). Reflex Sympathetic Dystrophy: A Reappraisal. Progress in Pain Management and Research. IASP Press; Seattle, 1996, pp 217-237.

Stanton-Hicks M. Complex regional pain syndrome (Type I, RSD; Type II, Causalgia): Controversies. Clin J Pain 2000; 16:S33-40.

Stav A, Ovadia L, Sternberg A et al. Cervical epidural steroid injection for cervicobrachialgia. Acta Anaesthesiol Scand 1993; 37:562-566.

Steindler A. Luck JV. Differential diagnosis of pain in the low back: Allocation of the source of the pain by the procaine hydrochloride method. JAMA 1938; 110:106-113.

Sternbach RA. Survey of pain in the United States. The Nuprin pain report. Clin J Pain 1986; 3:49-53.

Stewart HD, Quinnell RC, Dann N. Epidurography in the management of sciatica. Br J of Rheum 1987; 26:424-429.

Stitz MY, Sommer HM. Accuracy of blind versus fluoroscopically guided caudal epidural injection. Spine 1999; 24:13711376.

Strong, WE. Epidural abscess associated with epidural catheterization: A rare event? Report of two cases with markedly delayed presentation. Anesthesiology 1991; 74:943.

Suseki K, Takahashi Y, Takahashi K et al. Innervation of the lumbar facet joints. Spine 1997; 22:477-485.

Swerdlow M, Sayle-Creer W. A study of extradural medication in the relief of three lumbosciatic syndromes. Anaesthesia 1970; 25:341-345.

Tajima T, Furukawa K, Kuramochi E. Selective lumbosacral radiculography and block. Spine 1980; 1:68-77.

Teasell RW, Harth M. Functional restoration. Returning patients with chronic low back pain to work-Revolution or fad? Spine 1996; 21:844-847.

Tesfaye S, Watt J, Benbow SJ et al. Electrical spinal-cord stimulation for painful diabetic peripheral neuropathy. Lancet 1996; 348; 1698-1701.

The Executive Committee of the North American Spine Society. Position statement on discography. Spine 1988; 13:1343.

The Foundation for Health Services Research. Health Outcomes Research Primer. The Foundation for Health Services Research, Washington, 1994.

The quality indicator, June 2000, p10.

Thelander U. Fagerlung M., Friberg S et al. Straight leg raising test vs radiologic size, shape, and position of lumbar disc herniations. Spine 1992; 17:395-389.

Thomas E, Silman AJ, Croft PR et al. Predicting who develops chronic low back pain in primary care. A prospective study. Brit Med J 1999; 318:1662-1667.

Thompson RE, Pearcy MJ, Downing KJW. Disc lesions and the mechanics of the intervertebral joint complex. Spine 2000; 25:3026-3035.

Thomson SJ, Lomax DM, Collett BJ. Chemical meningism after lumbar facet joint block with local anaesthetic and steroids. Anaesthesia 1991; 46:563-564.
Tinker JH, Dull DL, Caplan RA. Role of monitoring devices in the prevention of anesthetic mishaps: A closed claim analysis. Anesthesiology 1989; 71:541-546.

Tolli TC, Cammisa FP Jr, Lane JM et al. Metastatic disease of the spine. Semin Spine Surg 1995;7:277-287.

Tosteson TD. Point of view. Spine 1999; 24:2562-2563.

Travell JG, Simons DG. Myofascial pain and dysfunction: The trigger point manual. Lippincott Williams \& Wilkins, Baltimore, 1999, Vol 2.

Triano JJ, McGregor M, Hondras MA et al. Manipulative therapy versus education programs in chronic low back pain. Spine 1995; 20:469-472.

Tulder MWV, Assendelft JJ, Koes BW et al. Method guidelines for systematic reviews in the Cochrane collaboration back review group for spinal disorders. Spine 1997; 22:2323-2330.

Tulder MWV, Cherkin DC, Berman B et al. The effectiveness of acupuncture in the management of acute and chronic low back pain. A systematic review within the framework of the Cochrane Collaboration Back Review Group. Spine 1999; 24:1113-1123.

Tulder MWV, Goossens M, Waddell G et al. Conservative treatment of chronic low back pain. In Nachemson AL, Jonsson E (eds). Neck and Back Pain. Lippincott Williams \& Wilkins, Philadelphia, 2000, pp 271-304.

Tulder MWV, Koes BW, Bouter LM. Conservative treatment of acute and chronic nonspecific low back pain. A systematic review of randomized controlled trials of the most common interventions. Spine 1997; 22:2128-2156.

Tulder MWV, Malmivaara A, Esmail R. Exercise therapy for low back pain. A systematic review within the framework of the Cochrane collaboration back review group. Spine 2000; 25:2784-2796.

Tulder MWV, Osteolo R, Vlaeyen JWS et al. Behavioral treatment for chronic low back pain. A systematic review within the framework of the Cochrane back review group. Spine 2000; 25:2688-2699.

Turk DC, Okifuji A. Treatment of chronic pain patients. Clinical outcomes, cost-effectiveness, and cost-benefits of multidisciplinary pain centers. Crit Rev Phys Rehabil Med 1998; 10:181-208.

Turk DC. Statistical significance and clinical significance are not synonyms! Clin J Pain 2000; 16:186-187.

Turk MA. Practice guidelines. In Gonzalez EG, Materson RS (eds). The nonsurgical management of acute low back pain. Demos Vermande, New York, 1997, pp 5-14.

Turner JA, Ersek M, Herron L et al. Surgery for lumbar spinal stenosis, attempted meta-analysis of the literature. Spine 1992; 17:1-8.

Turner JA, Ersek, Herron L et al. Patient outcomes after lumbar spinal fusions. JAMA 1992; 268:907-911.

Turner JA, Loeser JD, Bell KG. Spinal cord stimulation for chronic low back pain. A systematic literature synthesis. Neurosurgery 1995; 37:1088-1098.

Tutak U, Doleys DM. Intrathecal infusion systems for the treatment of chronic low back and leg pain of non-cancer origin. South Med J 1996; 89:295-300.

Van Akkerveeken PF. Lateral stenosis of the lumbar spine. Thesis. University of Utrecht, 1989. 
Van Akkerveeken PF. The diagnostic value of nerve root sheath infiltration. Acta Orthop Scand 1993; 64:61-63.

Van Den Hoogen HJM, Koes BW, Deville W et al. The prognosis of low back pain in general practice. Spine 1997; 22:15151521.

Van der Windt DAWM, van der Jeijden GJMG, van den Berg SGM et al. Ultrasound therapy for musculoskeletal disorders. A systematic review. Pain 1999; 81:257-271.

Van Kleef AR, Spaans F, Digemavs W et al. Effects and side effects of percutaneous thermal lesions of the dorsal root ganglion in patients with cervical pain syndromes. Pain 1993; 52:49-53.

Van Kleef M, Barendse GA. Percutaneous intradiscal radiofrequency thermocoagulation in chronic non-specific low back pain. Pain Clinic 1996; 3:259-268.

Van Kleef M, Barendse GAM, Kessels A et al. Randomized trial of radiofrequency lumbar facet denervation for chronic low back pain. Spine 1999; 24:1937-1942.

Van Kleef M, Liem L, Lousberg R et al. Radiofrequency lesions adjacent to the dorsal root ganglion for cervicobrachial pain. A prospective double blind randomized study. Neurosurgery 1996; 38:1-6.

Van Korff M, Dworkin SF, Le Resche L et al. An epidemiologic comparison of pain complaints. Pain 1988; 32:173-183.

van Poppel MN, Koes BW, van der Ploeg T et al. Lumbar supports and education for the prevention of low back pain in industry: A randomized controlled trial. JAMA 1998; 279:17891794.

Vanharanta H, Sachs BL, Spivey MA et al. The relationship of pain provocation to lumbar disc deterioration as seen by CT/ discography. Spine 1987; 12:295-298.

Veldman P, Reynen HM, Arntz IE et al. Signs and symptoms of reflex sympathetic dystrophy: Prospective study of 829 patients. Lancet 1993; 342:1012-1016.

Ventafridda GV, Caraceni AT, Sbanotto AM et al. Pain treatment in cancer of the pancreas. Eur J Surg Oncol 1990; 16:1-6.

Vervest ACM, Stolker RJ. The treatment of cervical pain syndromes with radiofrequency procedures. Pain Clinic 1991; 4:103-112.

Viner N. Intractable sciatica-The sacral epidural injection-An effective method of giving pain relief. Can Med Asso J 1925; 15:630-634.

Vogel JB III, Brown WH, Helms CA et al. The normal sacroiliac joint: A CT study of asymptomatic patients. Radiol 1984; 151:433-437.

Vogelsang H. Discitis intervertebralis cervicalis nach diskographie. Neurochirurgia 1973; 16:80-83.

Vulink N, Overgaauw DM, Jessurun G et al. The effects of spinal cord stimulation on quality of life in patients with therapeutically chronic refractory angina pectoris. Neuromodulation 1999; 2:33-40.

Waddell G, Gibson NA, Grant I. Surgical treatment of lumbar disc prolapse and degenerative lumbar disc disease. In Nachemson AL, Jonsson E (eds). Neck and Back Pain. Lippincott Williams \& Wilkins, Philadelphia, 2000, pp 305-325.

Waddell G, Kummel EG, Lotto WN et al. Failed lumbar disc surgery and repeat surgery following industrial injury. $J$ Bone Joint Surg (Am) 1979; 61:201-207.

Waddell G, Turk DC. Clinical assessment of low back pain. In Turk DC, Melzack R (eds). Handbook of pain assessment. First Edition. The Guilford Press, New York, 1992, pp 15-36.

Waldman SD, Waldman KA, Siwek SM. Interventional pain management, programming for success: In Waldman SD (ed), Interventional pain management. Second Edition. W.B. Saunders Company, Philadelphia 2000, pp 79-82.

Waldman SD. Cervical epidural abscess after cervical epidural nerve block with steroids (Letter). Anesth Anal 1991; 72:717.

Waldman SD. Complications of cervical epidural nerve blocks with steroids: A prospective study of 790 consecutive blocks. Reg Anesth 1989; 14:149-151.

Wallis BJ, Lord SM, Bogduk N. Resolution of psychological distress of whiplash patients following treatment by radiofrequency neurotomy: A randomized, double-blind, placebo-controlled trial. Pain 1997; 73:15-22.

Walsh TR, Weinstein JN, Spratt KP et al. Lumbar discography in normal subjects. J Bone Joint Surg 1990; 72A:1081-1088.

Wang LP, Haverberg J, Schmidt JF. Incidence of spinal epidural abscess after epidural analgesia. Anesthesiology 1999; 91:19281936.

War SE, Gordon D. Application of the American Pain Society quality assurance standards. Pain 1994; 56:299-306.

Warfield CA, Biber MP, Crews DA et al. Epidural steroid injection as a treatment for cervical radiculitis. Clin J Pain 1988; 4:201-204.

Warr AC, Wilkinson JA, Burn JMB et al. Chronic lumbosciatica syndrome treated by epidural injection and manipulation. Practitioner 1977; 209:53-59.

Wassell JT, Gardner LI, Landsittel DP et al. A prospective study of back belts for prevention of back pain and injury. JAMA 2000; 284:2727-2732.

Watts RW, Silagy CA. A meta-analysis on the efficacy of epidural corticosteroids in the treatment of sciatica. Anaesth Intens Care 1995; 23:564-569.

Weber H. Lumbar disc herniation: Controlled, prospective study with ten years of observation. Spine 1983; 8:131-140.

Weber $\mathrm{H}$. The natural history of disc herniations and the influence of intervention. Spine 1994; 19:2234-2238.

Wedel DJ, Wilson PR. Cervical facet arthrography. Reg Anesth 1985; 10:7-11.

Weiner BK, Fraser RD. Foraminal injection for lateral lumbar disc herniation. J Bone Joint Surg 1997; 79-B:804-807.

Weinstein JN. The tortoise and the hare. Is there a place in spine surgery for randomized trials? Spine 1999; 23:2548-2549.

Weinstein SM, Herring SA, Derby R. Epidural steroid injections. Spine 1995; 20:1842-1846.

Wertheim HM, Rovenstine EA. Suprascapular nerve block. Anesthesiology 1941; 2:541.

Wetzel FT, Andersson G, Pezola JH et al. Intradiscal electrothermal annuloplasty (IDET) to treat discogenic low back pain: Preliminary results of a multi-center. Cohort study: Proceedings of North American Spine Society, 15 ${ }^{\text {th }}$ Annual Meeting, New Orleans, 2000, pp 195-197. 
Wetzel FT, Hassenbusch S, Oakley JC et al. Treatment of chronic pain in failed back surgery patients with spinal cord stimulation: A review of current literature and proposal for future investigation. Neuromodulation 2000; 3:59-74.

Wheeler AH, Hanley EN Jr. Nonoperative treatment for low back pain. Rest to restoration. Spine 1995; 20:375-378.

White AH, Derby R, Wynne G. Epidural injections for diagnosis and treatment of low back pain. Spine 1980; 5:78-86.

White JC. Diagnostic novocaine block of the sensory and sympathetic nerves. Am J Surg 1930; 9:264.

Wilkinson H. Neurosurgical procedures of the sympathetic nervous system. Pain Clinic 1995; 1:43-50.

Wilkinson H. Percutaneous radiofrequency, upper thoracic sympathectomy. New Technique. Neurosurgery 1984; 15:811814.

Wilkinson HA. Introduction: Etiology, diagnosis, and therapy. In The Failed Back Syndrome. Etiology and therapy, Second Edition. Springer-Verlag, New York, 1992, pp 1-3.

Wilkinson HA. The role of improper surgery in the etiology of the failed back syndrome. In: The Failed Back Syndrome. Etiology and therapy, Second Edition. Springer-Verlag, New York, 1992, pp 4-12.

Williams KN, Jackowski A, Evans PJ. Epidural haematoma requiring surgical decompression following repeated cervical epidural steroid injections for chronic pain. Pain 1990; 42:197199.

Williamson JA. Inadvertent spinal subdural injection during attempted spinal epidural steroid therapy. Anaesth Intens Care 1990; 18:406-408.

Willis KD, Doleys DM. The effects of long-term intraspinal infusion therapy with noncancer pain patients: Evaluation of patient, significant - other, and clinic staff appraisals. Neuromodulation 1999; 2:241-253.

Wilson IB, Kaplan S. Clinical practice and patient health status: How are the two related? Med Care 1995; 33:AS209-AS214.

Winkelmüller M, Winkelmüller F. Long-term effects of continuous intrathecal opioid treatment in chronic pain of non-malignant etiology. J Neurosurg 1996; 85:458-467.

Winnie AP, Hartman JT, Meyers HL et al. Pain Clinic II: Intradural and extradural corticosteroids for sciatica. Anesth Analg 1972; 51:990-1003.

Winter RB. The prospective, randomized, controlled clinical trial in spine surgery. Fact or fiction? Spine 1999; 23:25502552.
Wolfe F. The relation between tender points and fibromyalgia symptom variables: evidence that fibromyalgia is not a discrete disorder in the clinic. Ann Rheum Dis 1997; 56:268-271.

Wong JB, Sonnenberg FA, Salem DN et al. Myocardial revascularization for chronic stable angina: Analysis of the role of percutaneous transluminal coronary angioplasty based on data available in 1989. Ann Intern Med 1990; 113:852-871.

Woo J, Ho SC, Lau J et al. Musculoskeletal complaints and associated consequences in elderly Chinese ages 70 years and over. J Rheumatol 1994; 21:1927-1931.

Woolf SH. Practice guidelines, a new reality. Medicine II. Methods of developing guidelines. Arch Intern Med 1992; 152:946952.

Woolf SH. Practice guidelines: A new reality in medicine. I. Recent developments. Arch Intern Med 1990; 150:1811-1818.

Yabuki S, Igarashi T, Kikuchi S. Application of nucleus pulposus to the nerve root simultaneously reduces blood flow in dorsal root ganglion and corresponding hindpaw in the rat. Spine 2000; 25:1471-1476.

Yabuki S, Kikuchi S, Olmarker K et al. Acute effects of nucleus pulposus on blood flow and endoneurial fluid pressure in rat dorsal root ganglia. Spine 1998; 23:2517-2523.

Yaeger B, Shootsky SA. Double-blind, controlled study of different myofascial trigger point injection techniques. Pain 1987; 31:5292.

Yamazaka N. Interspinal injection of hydrocortisone or prednisolone in the treatment of intervertebral disc herniation. JJpn Orthop Assoc 1959; 33:689.

Yap KB, Finaly IG. Epidural infection associated with epidural catheterization in a cancer patient with back pain: Case report. Palliat Med 1994; 8:251-253.

Yates DW. A comparison of the types of epidural injection commonly used in the treatment of low back pain and sciatica. Rheum Rehab 1978; 17:181-186.

Zakrzewska JM, Hamlyn PJ. Facial pain. In Crombie IK, Croft PR, Linton SJ et al (eds), Epidemiology of pain. IASP Press, Seattle, 1999, pp 171-202.

Zappala G. Iniezione peridurale segmentaria di Hydrocortone nella sindrome dolorosa da ernia discale. Policlinico - Sez Prat $1955 ; 62: 1229-1231$. 